SANDIA REPORT

SAND96-0819 • UC-814

Unlimited Release

Printed September 1996

Yucca Mountain Site Characterization Project

RECEIVED

NOV 041996

OSTI

\title{
Flow Calculations for Yucca Mountain Groundwater Travel Time (GWTT-95)
}

S. J. Altman, B. W. Arnold, R. W. Barnard, G. E. Barr,

C. K. Ho, S. A. McKenna, R. R. Eaton

Prepared by

Sandia National Laboratories

Albuquerque, New Mexico 87185 and Livermore, California 94550

for the United States Department of Energy

under Contract DE-AC04-94AL 85000

Approved for public release; distribution is unlimited.

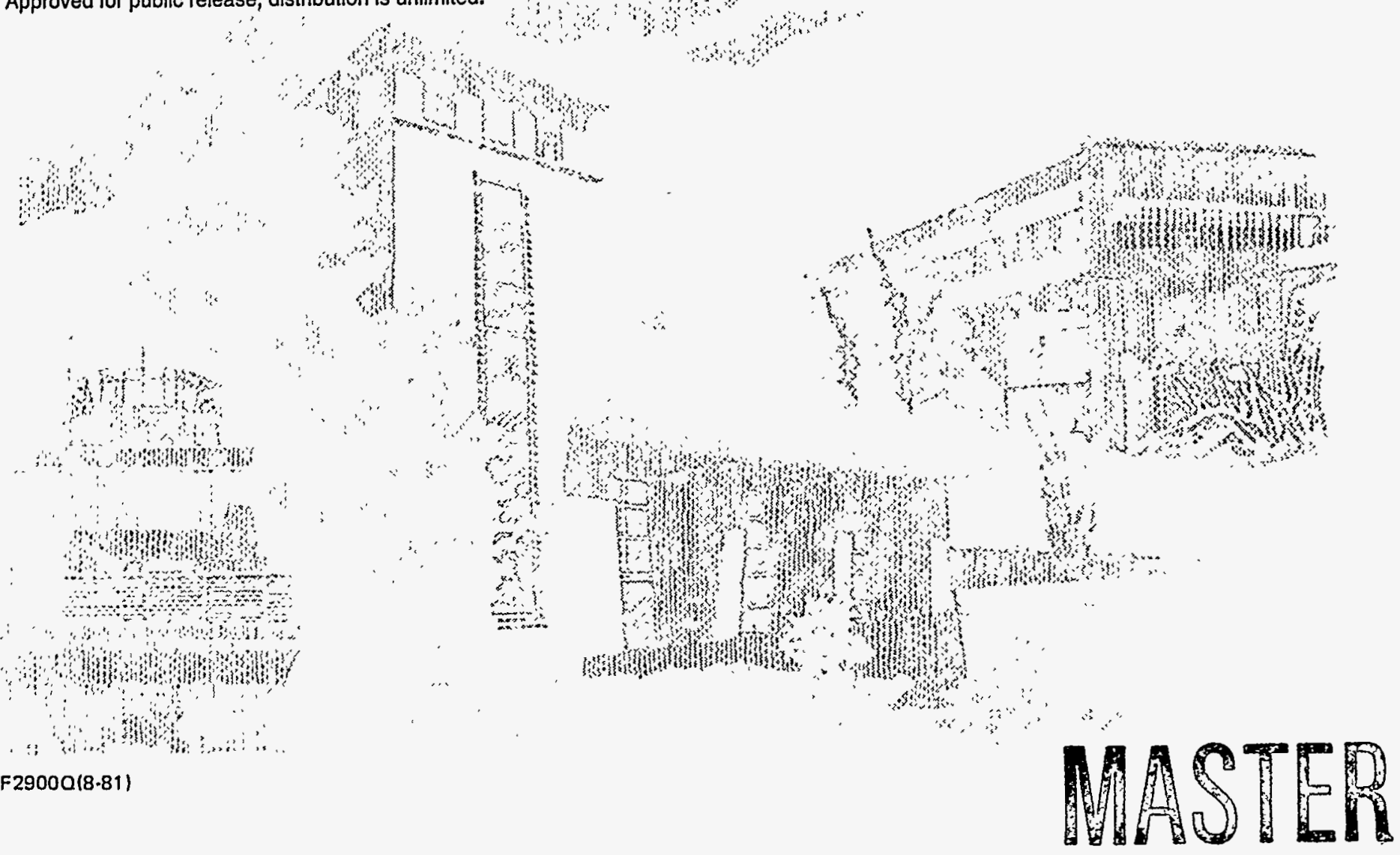


"Prepared by Yucca Mountain Site Characterization Project (YMSCP) participants as part of the Civilian Radioactive Waste Management Program (CRWM). The YMSCP is managed by the Yucca Mountain Project Office of the U.S. Department of Energy, DOE Field Office, Nevada (DOE/NV). YMSCP work is sponsored by the Office of Geologic Repositories (OGR) of the DOE Office of Civilian Radioactive Waste Management (OCRWM)."

Issued by Sandia National Laboratories, operated for the United States Department of Energy by Sandia Corporation.

NOTICE: This report was prepared as an account of work sponsored by an agency of the United States Government. Neither the United States Government nor any agency thereof, nor any of their employees, nor any of their contractors, subcontractors, or their employees, makes any warranty, express or implied, or assumes any legal liability or responsibility for the accuracy, completeness, or usefulness of any information, apparatus, product, or process disclosed, or represents that its use would not infringe privately owned rights. Reference herein to any specific commercial product, process, or service by trade name, trademark, manufacturer, or otherwise, does not necessarily constitute or imply its endorsement, recommendation, or favoring by the United States Government, any agency thereof or any of their contractors or subcontractors. The views and opinions expressed herein do not necessarily state or reflect those of the United States Government, any agency thereof or any of their contractors.

Printed in the United States of America. This report has been reproduced directly from the best available copy.

Available to DOE and DOE contractors from

Office of Scientific and Technical Information

PO Box 62

Oak Ridge, TN 37831

Prices available from (615) 576-8401, FTS 626-8401

Available to the public from

National Technical Information Service

US Department of Commerce

5285 Port Royal Rd

Springfield, VA 22161

NTIS price codes

Printed copy: A05

Microfiche copy: A01 
Distribution

Category UC-814

SAND96-0819

Unlimited Release

Printed September 1996

\title{
FLOW CALCULATIONS FOR YUCCA MOUNTAIN GROUNDWATER TRAVEL TIME (GWTT-95)
}

\author{
S. J. Altman, B. W. Arnold, R. W. Barnard, and G. E. Barr \\ YMP Performance Assessment Department \\ C. K. Ho and S. A. McKenna \\ Geohydrology Department \\ R. R. Eaton \\ Manufacturing and Environmental Fluid Dynamics Department
}

Sandia National Laboratories

Albuquerque, NM 87185

\begin{abstract}
In 1983, high-level radioactive waste repository performance requirements related to groundwater travel time were defined by NRC subsystem regulation 10 CFR 60.113. Although the DOE is not presently attempting to demonstrate compliance with that regulation, understanding of the prevalence of fast paths in the groundwater flow system remains a critical element of any safety analyses for a potential repository system at Yucca Mountain, Nevada. Therefore, this analysis was performed to allow comparison of fast-path flow against the criteria set forth in the regulation. Models developed to describe the conditions for initiation, propagation, and sustainability of rapid groundwater movement in both the unsaturated and saturated zones will form part of the technical basis for total-system analyses to assess site viability and site licensability.


This study is an enhancement of previous groundwater travel time analyses. In it the authors attempt to define the appropriate conceptual model for assessing fast-path flow. The conceptual model thus allows disequilibrium flow in the fractures and in the matrix. This conceptual model also allows for spatial and temporal variability of infiltration from the ground surface. In addition, the natural heterogeneity in hydrologic properties and the uncertainty in the values for those properties is expected to play an important role in allowing controlling rapid movement of groundwater. Because of this uncertainty in the distribution of rock properties, a probabilistic approach has been used in the unsaturated zone. Geostatistical simulation techniques, constrained by a geologic framework model, are used to create multiple realizations of the unsaturated domain. Numerical modeling of steady-state groundwater flow in these heterogeneous, two-dimensional domains is accomplished using the TOUGH2 code. A particle tracking method is used to simulate the advective and dispersive movement of groundwater in the simulated flow field and to calculate travel times from the repository to the water table. Numerical modeling of steady-state groundwater flow in the saturated zone is performed within a deterministic, three-dimensional model domain representing the upper $250 \mathrm{~m}$ of the saturated zone. Advective and dispersive transport are simulated by particle tracking from the water table to the five $\mathrm{km}$ limit of the accessible environment.

The results of the groundwater flow modeling provide insights into the implications of the conceptual models employed, the sensitivity of modeling results to specific parameters and assumptions, and distributions of groundwater travel time for a set of base case calculations. Values of matrix saturation simulated by the unsaturated flow modeling compare favorably with measured matrix saturations from boreholes near the modeled cross-sections. Particle travel times in the unsaturated zone are particularly sensitive to infiltration rates, fracture-matrix connectivity, and fracture frequency. Increased infiltration rate, in concert with reduced fracturematrix connectivity, results in short travel times, while simulating generally realistic matrix saturations. The presence or absence of simulated lateral diversion of groundwater flow in the non-welded PTn unit above the repository is shown to be sensitive to.the van Genuchten moisture characteristic curve parameters used in the flow modeling. The introduction of a large, transient infiltration event at the surface results in the relatively rapid propagation of increased fracture flow velocity within the mountain in the unsaturated flow model. For the base-case calculations of flow in the unsaturated zone, particle travel times to the water table vary from approximately 50 years to greater than 1,000,000 years, with the faster travel times occurring from the southern end of the repository. In the saturated zone, the flow model is calibrated to hydraulic head measurements in wells by the inclusion of four faults as low hydraulic conductivity zones. The distributions of particle travel times to the five $\mathrm{km}$ limit in the saturated zone vary dramatically depending on the location where the particles are released beneath the repository. Particle travel times in the saturated zone vary from about 250 years to 1,000,000 years, with the faster travel times occurring for particles released beneath the southern and western parts of the repository. There is considerable uncertainty in the absolute values of travel times in the saturated zone due to uncertainty in hydraulic conductivities of units downgradient of the repository, but the relative pattern of travel times is probably valid. Distributions of total combined groundwater travel times in the unsaturated zone and saturated zone exceed 1,000 years at four of five locations considered from the potential repository. Approximately $40 \%$ of the particles released from one location at the southern portion of the repository have total simulated travel times of less than the regulatory 


\section{DISCLAIMER}

Portions of this document may be illegible in electronic image products. Images are produced from the best available original document. 


\section{DISCLAIMER}

This report was prepared as an account of work sponsored by an agency of the United States Government. Neither the United States Government nor any agency thereof, nor any of their employees, makes any warranty, express or implied, or assumes any legal liability or responsibility for the accuracy, completeness, or usefulness of any information, apparatus, product, or process disclosed, or represents that its use would not infringe privately owned rights. Reference herein to any specific commercial product, process, or service by trade name, trademark, manufacturer, or otherwise does not necessarily constitute or imply its endorsement, recommendation, or favoring by the United States Government or any agency thereof. The views and opinions of authors expressed herein do not necessarily state or reflect those of the United States Government or any agency thereof. 
limit of 1,000 years. There are important uncertainties in this analysis that preclude definitive evaluation of regulatory compliance.

The results of this study support our assumptions regarding the importance of both selecting an appropriate conceptual model of groundwater flow processes and the incorporation of heterogeneity in material properties into the analyses. The modeling assumptions employed in the analyses are supported by favorable comparison of simulated matrix saturation values with measurements at the site. Simulated particle velocities are also qualitatively substantiated by recent isotopic dating measurements. This study also shows that the groundwater travel times are sensitive to variation and uncertainty in hydrologic parameters and in the infiltration flux at the upper boundary of the problem domain. Simulated travel times are also highly sensitive to poorly constrained parameters describing the interaction between flow in fractures and in the matrix.

This work was supported by the United States Department of Energy under Contract DEAC04-94AL85000, and was performed under WBS 1.2.5.4.4.

The data in this report were developed subject to the controls in QAGR 042, Revision 0, PCA 2.0, Task 2.1, and was done under Work Agreement WA-0181, Revision 00. The data used as input or reported are not qualified. The lack of qualified, spatially distributed data for some critical parameters necessitated the inclusion of nonqualified data in the analysis. 


\section{Acknowledgments}

The authors would like to thank those individuals who contributed to the success of this study. Christopher Rautman (Sandia National Laboratories), Marc Cromer and William Zelinski (Spectra Research Institute) were instrumental in developing the GLINTMOD software link between the geologic model of the unsaturated zone and the geostatistical simulation algorithms used in this work. Al Schenker (Los Alamos Technical Associates) assisted in the acquisition and analysis of data on hydrologic parameters. Lorrie Flint (U. S. Geological Survey) provided recently acquired data on hydrologic parameters. Alan Flint (U. S. Geological Survey) supplied pre-publication estimates of the spatial distribution of surface infiltration. Ed Kwicklis and Ning Lu (U. S. Geological Survey) graciously provided particle tracking software for use in conjunction with flow simulations in the unsaturated zone. Sharon Shannon (Sandia National Laboratories) produced many of the visualization graphics used in figures. Lee Skinner (Spectra Research Institute) and Paul van Gulick (Geo-Centers, Incorporated) developed particle tracking software used in the saturated zone calculations. Mike Wilson and Jim Duguid provided technical review of this document. Isaac Block, Carol Crawford, and Luke Davis assisted in editorial tasks and the preparation of the final document. Management of the project and management review of the document were contributed by Holly Dockery (Sandia National Laboratories). 


\section{Table of Contents}

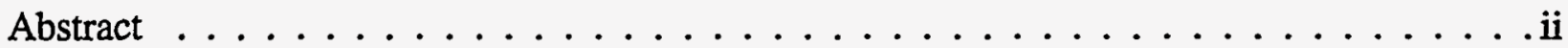

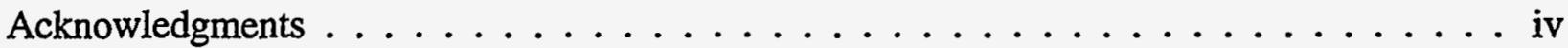

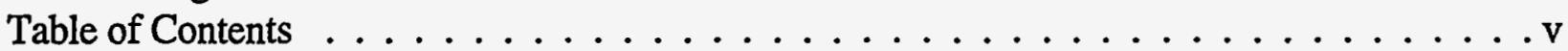

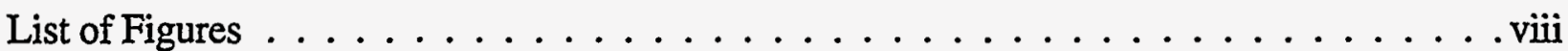

List of Tables $\ldots \ldots \ldots \ldots \ldots \ldots \ldots \ldots \ldots \ldots \ldots \ldots \ldots \ldots \ldots \ldots$

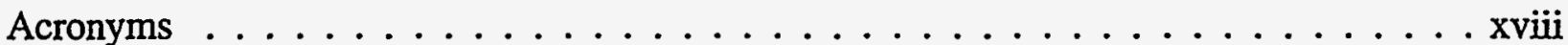

Executive Summary $\ldots \ldots \ldots \ldots \ldots \ldots \ldots \ldots \ldots \ldots \ldots \ldots \ldots \ldots$ xix

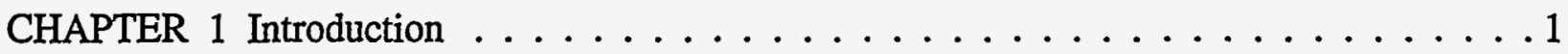

1.1 Regulatory Issues . . . . . . . . . . . . . . . . . .

1.2 NRC technical exchanges . . . . . . . . . . . . . . .

1.3 Overview of the GWTT-95 Study $\ldots \ldots \ldots \ldots \ldots \ldots \ldots$

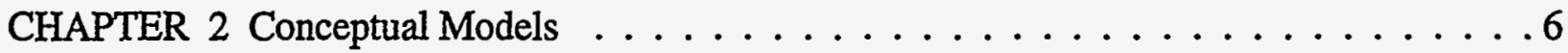

2.1 Yucca Mountain Site Hydrogeologic System . . . . . . . . . . . . 6

2.1 .1 Geology . . . . . . . . . . . . . . . . . .6 6

2.1 .2 Surface Hydrology . . . . . . . . . . . . . . . . .

2.1.3 Subsurface Hydrology . . . . . . . . . . . . . . . . .99

2.1.3.1 Unsaturated Zone . . . . . . . . . . . . .99

2.1.3.2 Saturated Zone . . . . . . . . . . . . . . . . 9

2.2 Conceptual Models of the Hydrogeologic System . . . . . . . . . . . 10

2.2 .1 Geology . . . . . . . . . . . . . . . . . 11

2.2 .2 Unsaturated Zone . . . . . . . . . . . . . . . 11

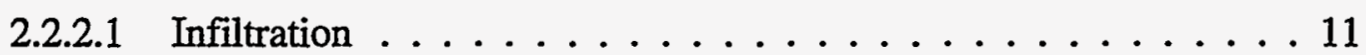

2.2.2.2 Flow Processes . . . . . . . . . . . . . . . 12

2.2 .3 Saturated Zone . . . . . . . . . . . . . . . . . . . . . . 13

2.3 Alternative Implementations of Conceptual Flow Models . . . . . . . . 13

2.3.1 Overview of Conceptual Flow Models . . . . . . . . . . . . . . 13

2.3.2 Comparison of the ECM and DKM Conceptual Models . . . . . . . . 15

2.4 Conceptual Models Employed in the GWTT-95 Analysis . . . . . . . . . . . . 17

2.4 .1 Unsaturated Zone . . . . . . . . . . . . . . . . . . . 18

2.4.1.1 Geology . . . . . . . . . . . . . . . 18

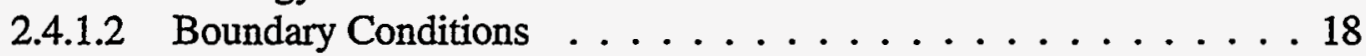

2.4.1.3 Flow Processes . . . . . . . . . . . . . . . 18

2.4 .2 Saturated Zone . . . . . . . . . . . . . . . . . 19

2.4.2.1 Geology . . . . . . . . . . . . . . . . 19

2.4.2.2 Boundary Conditions . . . . . . . . . . . . 19

2.4.2.3 Flow Processes . . . . . . . . . . . . . . . . 19

CHAPTER 3 Parameter and Geological Model Development . . . . . . . . . . 20

3.1 Geologic Framework Models . . . . . . . . . . . . . . . . . 20

3.1 .1 Unsaturated Zone . . . . . . . . . . . . . . . . . . . 20

3.1 .2 Saturated Zone . . . . . . . . . . . . . . . . . . 26

3.2 Hydrological Parameter Data Synthesis and Analysis . . . . . . . . . . . 26 
3.2.1 Matrix Parameters . . . . . . . . . . . . . . 28

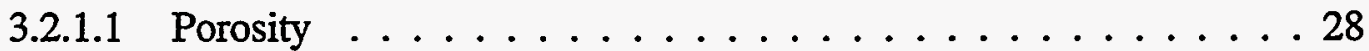

3.2.1.2 Saturated Hydraulic Conductivity . . . . . . . . . . 28

3.2.1.3 Characteristic Curve Parameters . . . . . . . . . . . . . 29

3.2.2 Fracture Properties . . . . . . . . . . . . . . . . . . 37

3.2.2.1 Fracture Frequency $\ldots \ldots \ldots \ldots \ldots \ldots$

3.2.2.2 Fracture Porosity . . . . . . . . . . . . . . . 40

3.2.2.3 Fracture Aperture and Permeability . . . . . . . . . . 43

3.2.2.4 Characteristic Curve Parameters . . . . . . . . . . . . 43

3.2.2.5 Fracture Network Dispersivity . . . . . . . . . . . . . . 44

3.3 Geostatistical Simulation of Hydrologic Properties in the Unsaturated Zone . . . . . 47

3.3.1 Link to Geologic Framework Model . . . . . . . . . . . . . . . . 47

3.3.2 Gaussian Simulation . . . . . . . . . . . . . . . . . 48

3.3.2.1 Matrix Porosity . . . . . . . . . . . . . . . 48

3.3.2.2 Coregionalization of Matrix Saturated Hydraulic Conductivity . . . 54

3.3.2.3 Fracture Frequency . . . . . . . . . . . . . 59

3.3.3 Indicator Simulation of Tectonic Fracture Zones. . . . . . . . . . . 60

3.3.4 Upscaling of Hydrologic Properties . . . . . . . . . . . . . . 62

3.3.5 Derived Hydrologic Properties . . . . . . . . . . . . . . . 65

3.4 Summary . . . . . . . . . . . . . . . . . . . 67

3.4 .1 Evaluation of Rock Properties Models . . . . . . . . . . . . . . 67

3.4 .2 Discussion. . . . . . . . . . . . . . . . . . . . 74

CHAPTER 4 Numerical Flow Modeling in the Unsaturated Zone $\ldots \ldots$. . . . . . 75

4.1 Boundary Conditions . . . . . . . . . . . . . . . . 75

4.2 Numerical Simulations of Flow . . . . . . . . . . . . . . 76

4.2 .1 Governing Equations . . . . . . . . . . . . . . . 76

4.2 .2 Numerical Grid . . . . . . . . . . . . . . . . . . . 80

4.2 .3 Numerical Solution Using TOUGH2 . . . . . . . . . . . . . . . 81

4.3 Flow Modeling Results and Discussion . . . . . . . . . . . . . . . . 82

4.3.1 Matrix Saturations . . . . . . . . . . . . . . . 82

4.3.2 Fracture Saturations. . . . . . . . . . . . . . . .91

4.3 .3 Flow Velocities . . . . . . . . . . . . . . . . .91

4.4 Particle Tracking . . . . . . . . . . . . . . . . . . . . . . 98

4.4.1 Particle Transport Processes . . . . . . . . . . . . . . . . . 98

4.4.2 Implementation of Particle Tracking . . . . . . . . . . . . . 99

4.4 .3 Particle Tracking Results . . . . . . . . . . . . . . . . . . . . 100

4.5 Sensitivity Analysis . . . . . . . . . . . . . . . . . . . . . . . . . . . .

4.5.1 Infiltration Flux. . . . . . . . . . . . . . . . . 105

4.5.2 Fracture-Matrix Connectivity Scaling . . . . . . . . . . . . . . 107

4.5 .3 Fracture Frequency . . . . . . . . . . . . . . . . . . . . . 108

4.5.4 Fracture Residual Saturation . . . . . . . . . . . . . . . . . 114

4.5 .5 Zeolitic Region . . . . . . . . . . . . . . . . . . . . . 117

4.5.6 Numerical Grid . . . . . . . . . . . . . . . . . . . . . . . . . . 119

4.5.7 Equivalent Continuum Model . . . . . . . . . . . . . . 123

4.5.8 Matrix van Genuchten Parameters . . . . . . . . . . . . . . . . . 124 
4.5 .9 Episodic Infiltration. . . . . . . . . . . . . . . . . . . 128

CHAPTER 5 Numerical Flow Modeling In The Saturated Zone . . . . . . . . . 136

5.1 Flow Model Domain . . . . . . . . . . . . . . . . . . . . . 136

5.1 .1 Hydrostratigraphic Units . . . . . . . . . . . . . . . . 136

5.1 .2 Boundary Conditions . . . . . . . . . . . . . . . 138

5.2 Numerical Simulation of Flow . . . . . . . . . . . . . . . . . . . . . . . . . . . . . . . . . . .

5.2 .1 Governing Equations . . . . . . . . . . . . . . . 138

5.2 .2 Numerical Grid . . . . . . . . . . . . . . . . . . . . 139

5.2.3 Numerical Solution Using STAFF3D . . . . . . . . . . . . . 139

5.3 Flow Model Calibration and Results . . . . . . . . . . . . . . . . . . . . . 139

5.4 Particle Tracking . . . . . . . . . . . . . . . . . . . . 145

5.4.1 Particle Transport Processes . . . . . . . . . . . . . . . 145

5.4.2 Implementation of Particle Tracking . . . . . . . . . . . . 145

5.4.3 Particle Tracking Results . . . . . . . . . . . . . . . . . . . 146

CHAPTER 6 Results, Conclusions, Future Work, and Recommendations . . . . . . . . . 152

6.1 Results . . . . . . . . . . . . . . . . . . . 152

6.1 .1 Unsaturated Zone . . . . . . . . . . . . . . . . . . . 152

6.1 .2 Saturated Zone . . . . . . . . . . . . . . . . . . 153

6.1.3 Combined Unsaturated and Saturated Zone Travel Times . . . . . . . . 154

6.2 Conclusions . . . . . . . . . . . . . . . . . . . . 157

6.3 Implications With Regard To GWTT Regulations . . . . . . . . . . . . 160

6.4 Future Directions . . . . . . . . . . . . . . . . . . . . . 161

6.5 Recommendations . . . . . . . . . . . . . . . . . 161

References . . . . . . . . . . . . . . . . . . . . . . . 163 


\section{List of Figures}

Figure 2-1: Schematic cross-section of the potential Yucca Mountain repository region. $\ldots .8$

Figure 2-2: Alternative conceptual models and their corresponding relative permeability characteristic curves for flow through fractured rock. . . . . . . . . . 14

Figure 2-3: Mass flow between fracture and matrix elements in a dual-permeability model. The fracture-matrix conductance depends, in part, on the effective connection area, which may be reduced as a result of small scale processes such as fingering, preferential flow paths, and mechanical aspects such as fracture coatings. See Section 4.2 .1 for definitions of other variables. . . . . . . . . . . 17

Figure 3-1: Map showing location of borings from which matrix porosity and/or fracture frequency conditioning data were determined. The polygon defining the outline of the potential repository is shown along with the GWTT-95 cross-sections. . . . 21

Figure 3-2: Distributions of porosity for each hydrogeological unit. The numbers refer to the numbers used in Table 3-1. The thick dashed lines indicate the mean of each distribution and the thin dashed lines indicate the median. The 25th and 75th percentiles are defined by the thick boxes and the 10th and 90th percentiles are defined by the thin boxes. . . . . . . . . . . . . . 23

Figure 3-3: Geologic framework model for cross-sections AA (a) and BB (b). The color-scale for the hydrogeological units refers to the hydrogeological unit numbers used in Table 3-1. The area inside the dashed line indicates the region of zeolitization. 24

Figure 3-4: Geologic framework model for cross-sections CC (a) and DD (b). The color-scale for the hydrogeological units refers the hydrogeological unit numbers used in Table 3-1. The area inside the dashed line indicates the region of zeolitization. . . . . 25

Figure 3-5: Geologic model of the saturated zone shown for three layers, as incorporated into the flow model of the saturated zone. Asterisks indicate wells. . . . . . . . 27

Figure 3-6: Regression relationships of saturated hydraulic conductivity to matrix porosity used in the GWTT-95 calculations for (a) non-zeolitic units and (b) zeolitic units. . . 30

Figure 3-7: Distribution of van Genuchten $\alpha$ for the upper three hydrogeological units (TCw, PTn and TSw) from three different sources (data for TCw were not available from Rutherford et al., 1992). Note that the approximate mean $\log (\alpha$ 's) are higher in the data from Flint et al. (1996) and Flint (see footnote 10, page 29) than the other two references. .............................

Figure 3-8: Distribution of van Genuchten $\beta$ for the available data from three different sources. . . . . . . . . . . . . . . . . 33 
Figure 3-9: Comparison of the relationship of raw $\alpha$ data determined on laboratory samples to the average $\alpha$ 's calculated using in-situ and laboratory data. Values of lab data $\alpha$ 's are averages for the GWTT-95 hydrogeological units. Note the $\alpha$ 's in the nonwelded units are significantly higher after calibration. . . . . . . . . . 35

Figure 3-10: Regression relationship of matrix $\alpha$ to saturated hydraulic conductivity used for the GWTT-95 calculations. . . . . . . . . . . . . . . 36

Figure 3-11: Relationship of matrix $\beta$ to porosity. A constant $\beta$ value of 1.60 is used for the GWTT-95 calculations. . . . . . . . . . . . . . . . 37

Figure 3-12: Unsaturated characteristic curves for the TCw, PTn, and TSw using the mean matrix van Genuchten parameters, mean porosity and saturated hydraulic conductivity for each hydrostratigraphic unit. The lower graph (b) is an enlargement of the delineated area in the upper graph (a) . . . . . . . 38

Figure 3-13: Fracture frequency data before and after corrections for bias due to vertical boreholes and varying recovery rates for borehole NRG-6. . . . . . . . 41

Figure 3-14: Regression relationship between matrix and fracture porosity used for GWTT-95 calculations. This regression relationship is adjusted to preserve the fracture aperture distribution, as described in the text. . . . . . . . . . .42

Figure 3-15: Unsaturated characteristic curves for the TCw, PTn and TSw using mean fracture van Genuchten parameters used in GWTT-95 calculations. Note that the curves of units that are in contact with each other do not intersect indicating that lateral diversion would not occur in the fracture domain above or below the PTn in a system with homogeneous units. . . . . . . . . . . 45

Figure 3-16: Log probability plot and variogram of air permeability measurements in borehole UZ-16. . . . . . . . . . . . . . . . . . . . .

Figure 3-17: Resolution of the Solitario Canyon Fault at different ranges of spatial correlation. The fault as modeled in the deterministic framework model is shown in (a). Geostatistical simulation of porosity using GLINTMOD using a range of $460 \mathrm{~m}$ (b). The perpendicular to parallel anisotropy ratio is held at 0.01 for all realizations. 50

Figure 3-18: Resolution of the Solitario Canyon Fault at different ranges of spatial correlation. Porosity simulations created with a range $=1000 \mathrm{~m}$ (a) and $2000 \mathrm{~m}$ (b). The perpendicular to parallel anisotropy ratio is held at 0.01 for all realizations. . . .51

Figure 3-19: The relative number of references to the geologic framework model for simulation of porosity along section AA corresponding to the images in Figures $3-17$ and $3-18 . \ldots \ldots \ldots \ldots \ldots$. . . . . . . . . . . . .

Figure 3-20: Distribution of the matrix porosity conditioning data. The thick dashed line denotes the mean and the thin dashed line denotes the median. . . . . . . . . 53

Figure 3-21: Realization number one of porosity along cross-section AA. . . . . . . . . 54 
Figure 3-22: Unconditional, standard-normal realization number 1 at cross-section AA used in the coregionalization process. . . . . . . . . . . . 58

Figure 3-23: Realization number 1 of section AA for $\log _{10}$ matrix saturated hydraulic conductivity $(\mathrm{m} / \mathrm{s})$. This realization was produced through the model of linear coregionalization with porosity. Note the relatively lower (dark blue) conductivities in the zeolitized zone at the base of the section. . . . . . . . . . . . 59

Figure 3-24: Distribution of fracture frequency conditioning data in $\log _{10}$ (1/10feet). The thick dashed line denotes the mean value and the thin dashed line denotes the median value. . . . . . . . . . . . . . . . . . 60

Figure 3-25: Realization number 1 at section AA of the high fracture frequency zones created with indicator geostatistical simulation. The high fracture frequency zones are black and the areas of background fracture frequency are white. The mapped, deterministic fault features are labelled. . . . . . . . . . . . 61

Figure 3-26: Empirically derived relationship between matrix porosity and the fracture frequency multiplier. . . . . . . . . . . . . . . 62

Figure 3-27: Realization number 1 at cross-section $\mathrm{AA}$, of $\log _{10}$ (1/10feet) fracture frequency derived by combining realizations of cooling fractures with tectonically induced fracturing through the relationship defined in Figure $3-26 \ldots \ldots \ldots 3$

Figure 3-28: Computational grid for TOUGH2 used on cross-section AA. Properties are scaled from the geostatistical simulation scale to the computational scale. . . . . . . 64

Figure 3-29: Box and whisker plots of the porosity distributions for the ten realizations after being upscaled and for the porosity conditioning data. The thick and thin dashed lines denote the mean and median respectively. The boxes define the 10th, 25th, 75th and 90th percentiles and the ends of the whiskers are the minimum and maximum values. . . . . . . . . . . . . . . 65

Figure 3-30: Upscaled values of porosity shown in the computational grid. . . . . . . .66

Figure 3-31: Box and whisker plots of the distribution of Ksat for the ten realizations of crosssection AA after upscaling and the geostatistically simulated values for realization number one (INPUT \#1). The thick and thin dashed lines denote the mean and median respectively. The boxes define the 10 th, 25 th, 75 th and 90 th percentiles and the ends of the whiskers are the minimum and maximum values. . . . . . . . 67

Figure 3-32: Fracture permeability shown in the computational grid for cross-section AA, realization $1 . \ldots \ldots \ldots \ldots \ldots \ldots \ldots \ldots \ldots \ldots \ldots \ldots \ldots$

Figure 3-33: Comparison of porosity trends in boring SD-9 (A) with four vertical profiles taken along cross-section AA from porosity realization number one. The profiles were extracted at the easting coordinates: $170,000 \mathrm{~m}(B), 171,000 \mathrm{~m}(\mathrm{C}), 172,000 \mathrm{~m}(\mathrm{D})$

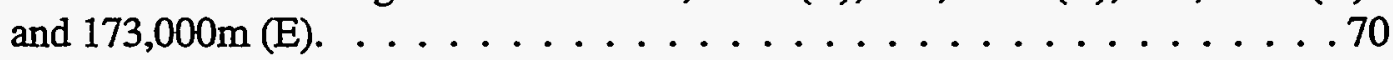


Figure 3-34: Regression relationship between simulated matrix porosity and Ksat for the nonzeolitized (a) and zeolitized (b) portions of the mountain resulting from the linear coregionalization and the regression models applied to the matrix porosity realization shown in Figure $3-6 . \ldots \ldots \ldots . \ldots . \ldots 72$

Figure 3-35: Expectation map of matrix porosity created by averaging the porosity on a pixel by pixel basis across 10 realizations created for cross-section AA. Compare the location and geometry of the regions of similar porosity to the locations and geometry of the hydrogeological units in Figure $3-3$ (a). . . . . . . .73

Figure 4-1: Infiltration rates and corresponding numerical grids for a) cross-section $A-A$ and $b$ ) cross-section B-B. . . . . . . . . . . . . . . . .77

Figure 4-1: Infiltration rates and corresponding numerical grids for $\mathrm{c}$ ) cross-section $\mathrm{C}-\mathrm{C}$ and d) cross-section D-D. . . . . . . . . . . . . . . 78

Figure 4-2a: Matrix and fracture saturations for cross-section AA, realization 1, dual permeability model. . . . . . . . . . . . . . 83

Figure 4-2b: Matrix and fracture saturations for cross-section $\mathrm{BB}$, realization 1, dual permeability model. . . . . . . . . . . . . . . . . . 84

Figure 4-2c: Matrix and fracture saturations for cross-section CC, realization 1, dual permeability model. . . . . . . . . . . . . . . . 85

Figure 4-2d: Matrix and fracture saturations for cross-section DD, realization 1, dual permeability model. . . . . . . . . . . . . . . 86

Figure 4-3: Simulated matrix saturations with depth at two columns of elements at the intersection of two cross-sections. . . . . . . . . . . . 88

Figure 4-4a: Comparisons of simulated matrix saturations to saturation measurements made from core samples from boreholes close to cross-sections AA and DD. . . . . . 89

Figure 4-4b: Comparisons of simulated matrix saturations to saturation measurements made from core samples from boreholes close to cross-sections BB and CC. . . . . 990

Figure 4-5a: Velocity vectors of flow through the fracture continuum for cross-sections AA and $B B$, realization 1, dual permeability model. All vectors in Figure 4-5 (crosssections AA, BB, CC, and DD) have the same scale. . . . . . . 992

Figure 4-5b: Velocity vectors of flow through the fracture continuum for cross-sections $\mathrm{CC}$ and DD, realization 1, dual permeability model. All vectors in Figure 4-5 (crosssections $\mathrm{AA}, \mathrm{BB}, \mathrm{CC}$, and $\mathrm{DD}$ ) have the same scale.. . . . . . . 993

Figure 4-6a: Distribution of Darcy velocities through the matrix domain for one realization of all four cross-sections. . . . . . . . . . . . . . . .94

Figure 4-6b: Distribution of Darcy velocities through the fracture domain for one realization of all four cross-sections. . . . . . . . . . . . . . . . . . 
Figure 4-7a: Flow between matrix and fracture continuums for cross-sections $\mathrm{AA}$ and $\mathrm{BB}$, realization 1 , dual permeability model. . . . . . . . . . . . 996

Figure 4-7b: Flow between matrix and fracture continuums for cross-sections CC and DD, realization 1 , dual permeability model. . . . . . . . . . . . . . 97

Figure 4-8: Ratio of fracture flux to total flux in the flow grid for the dual permeability simulation on cross-section AA, realization $1 \ldots \ldots \ldots \ldots . \ldots 98$

Figure 4-9a: Infiltration, simulated particle paths and groundwater travel times along crosssection AA. Particle paths for a single realization of material properties are plotted in the middle figure. Portions of particle paths in which particles are advected at fracture flow velocities are plotted in blue and where particles are advected at matrix flow velocities paths are plotted in red. Travel times are plotted in the lower graph for all ten realizations. . . . . . . . . . . . . . . 101

Figure 4-9b: Infiltration, simulated particle paths and groundwater travel times along crosssection BB. Particle paths for a single realization of material properties are plotted in the middle figure. Portions of particle paths in which particles are advected at fracture flow velocities are plotted in blue and where particles are advected at matrix flow velocities paths are plotted in red. Travel times are plotted in the lower graph for all ten realizations. . . . . . . . . . . . . . . . . . 102

Figure 4-9c: Infiltration, simulated particle paths and groundwater travel times along crosssection CC. Particle paths for a single realization of material properties are plotted in the middle figure. Portions of particle paths in which particles are advected at fracture flow velocities are plotted in blue and where particles are advected at matrix flow velocities paths are plotted in red. Travel times are plotted in the lower graph for all ten realizations. . . . . . . . . . . . . . . 103

Figure 4-9d: Infiltration, simulated particle paths and groundwater travel times along crosssection DD. Particle paths for a single realization of material properties are plotted in the middle figure. Portions of particle paths in which particles are advected at fracture flow velocities are plotted in blue and where particles are advected at matrix flow velocities paths are plotted in red. Travel times are plotted in the lower graph for all ten realizations. . . . . . . . . . . . . . . . . . 104

Figure 4-10: Comparison of simulated saturations using different spatially variable infiltration rates to measured core saturations from SD-9. . . . . . . . . . . . 106

Figure 4-11: Particle travel times along cross-section AA for flow simulations using different spatially variable infiltration rates. . . . . . . . . . . . . . 107

Figure 4-12: Comparison of simulated saturations to measured core saturations from SD-9 using a 3 order of magnitude and 5 order of magnitude reduction to full fracture-matrix connection and a mean infiltration rate of 0.19 and $1.9 \mathrm{~mm} / \mathrm{yrm}$ respectively. Note that both simulated saturation profiles match the in-situ saturations comparably well. . . . . . . . . . . . . . . . . . . . . . 109 
Figure 4-13: Comparison of simulated saturations to measured core saturations from SD-9 using different reductions to full fracture-matrix connection for a mean infiltration rate of (a) $0.19 \mathrm{~mm} / \mathrm{yr}$ and (b) $1.9 \mathrm{~mm} / \mathrm{yr}$. Note the decrease in matrix saturations with an increased reduction of fracture-matrix connectivity at both infiltration rates. . 110

Figure 4-14: Particle travel times along cross-section AA for flow simulations using different reductions to full fracture-matrix connection for a mean infiltration rate of (a) 0.19 $\mathrm{mm} / \mathrm{yr}$ and (b) $1.9 \mathrm{~mm} / \mathrm{yr}$. Note the larger effect of fracture-matrix connectivity on particle travel times at the lower infiltration rates. . . . . . . . . . 111

Figure 4-15: Particle travel times along cross-section AA for flow simulations using a 3 order of magnitude and 5 order of magnitude reduction to full fracture-matrix connection and a mean infiltration rate of 0.19 and $1.9 \mathrm{~mm} / \mathrm{yr}$, respectively. Note the almost two-order-of-magnitude difference in particle travel times despite the similar calculated saturations for the two simulations. . . . . . . . . . . 112

Figure 4-16: Corrected fracture frequency data from borehole NRG-6 using $10^{\circ}$ and $30^{\circ}$ increments of dip measurements for correction (see equation 3-1) . . . . 113

Figure 4-17: Regression relationship between matrix and fracture porosity using fracture frequency data using $30^{\circ}$ increments of dip measurements for correction used for GWTT-95 fracture frequency sensitivity study. This regression relationship is adjusted to preserve the fracture aperture distribution, as described in the Section 3.2.2.2. . . . . . . . . . . . . . . . . . . 114

Figure 4-18: Comparison of simulated saturations to measured core saturations from SD-9 for a reduced fracture frequency. Saturations values for a reduced fracture-matrix connectivity (see Section 4.5.2) are included for comparison. . . . . . . 115

Figure 4-19: Particle travel times along cross-section A-A for flow simulations using a reduced fracture frequency. Travel times for a reduced fracture-matrix connectivity (see Section 4.5.2) are included for comparison. . . . . . . . . . . 116

Figure 4-20: Particle travel times along cross-section AA for flow simulations using different fracture residual saturations. . . . . . . . . . . . . 117

Figure 4-21: Comparison of simulated saturations to measured core saturations from SD-9 for simulations with and without the hydrologic properties of a zeolitic region. . 118

Figure 4-22: Particle travel times along cross-section AA for flow simulations with and without the hydrological properties of a zeolitic region. . . . . . . . . . . . 119

Figure 4-25: Comparison of simulated saturations to measured core saturations from SD-9 comparing the base case to a simulation using a finer grid. . . . . . . 120

Figure 4-23: Matrix saturations for original and finer grid of cross-section AA. . . . . 121

Figure 4-24: Fracture saturations for original and finer grid of cross-section AA. . . . . 122 
Figure 4-26: Particle travel times along cross-section AA for base case flow simulation and a simulations using a finer grid. . . . . . . . . . . . . 123

Figure 4-27: Comparison of simulated saturations to measured core saturations from SD-9 for simulations using the equivalent continuum and the dual permeability model. 124

Figure 4-28: Comparison of particle travel times along cross-section $\mathrm{AA}$ for simulations using the equivalent continuum (with two different sets of van Genuchten $\alpha$ ) and dual permeability models. . . . . . . . . . . . . . . . 125

Figure 4-29: Relationship between Ksat and van Genuchten $\alpha$ used in simulating $\alpha$ 's using Klavetter's and Peters' data . . . . . . . . . . . . . . . . 126

Figure 4-30: Flow velocities through fractures for two simulations both using the equivalent continuum model, but one using GWTT-95 van Genuchten Parameters and the second using van Genuchten parameters from Klavetter and Peters (1986). . 127

Figure 4-31: Episodic infiltration rate applied to cross-section AA for one week. The effects of the episodic event is analyzed along a single column of elements that is outlined in bold. . . . . . . . . . . . . . . . . 128

Figure 4-32: Relative increase in fracture pore velocity, $v$, over the steady-state value, $v_{s s}$, as a function of elevation and time since the initiation of the infiltration pulse (note the $\log$ scale) for one column of elements as a result of the one week episodic infiltration event. . . . . . . . . . . . . . . . . . 129

Figure 4-33: Fracture pore velocity as a function of time at four locations along a column of elements in section AA as a result of a one-week episodic infiltration pulse. . 130

Figure 4-34: Fracture water content (liquid saturation times porosity) as a function of time at four locations along a column of elements in section AA as a result of a one-week episodic infiltration pulse. . . . . . . . . . . . 130

Figure 4-35: Matrix saturations as a function of time at four locations along a column of elements in section AA as a result of a one-week episodic infiltration pulse. . . . . . 131

Figure 4-36a: Particle movement through a single column of fracture elements as a result of a oneweek infiltration pulse in section AA. The plots show the position of water particles (denoted by symbols) at various times during and following the infiltration pulse. The fracture pore velocities are also shown to indicate the effects of the infiltration pulse. . . . . . . . . . . . . . . . 133

Figure 4-36b: Particle movement through a single column of fracture elements as a result of a oneweek infiltration pulse in section AA. The plots show the position of water particles (denoted by symbols) at various times during and following the infiltration pulse. The fracture pore velocities are also shown to indicate the effects of the infiltration pulse. . . . . . . . . . . . . . . . . . 134 
Figure 5-1: Areal extent of the saturated zone flow model (bold line) and locations of wells intersecting the water table. Contours indicate elevation of the topographic surface. Contour interval is $100 \mathrm{ft} . \ldots \ldots \ldots$. . . . . . . . . 137

Figure 5-2: Numerical grid for the STAFF3D flow model of the saturated zone. Water table wells used in the calibration of the model are shown with asterisks. Coordinates are based on the Nevada State Plane system. . . . . . . . . . . . . 140

Figure 5-3: Discrete structural features included in the calibrated SZ flow model. . . . 141

Figure 5-4: Simulated hydraulic head in the upper layer of the calibrated SZ flow model. The upper part of the figure shows the potentiometric surface over the entire model in perspective view. The lower figure is a contour plot of head only in the region of the low hydraulic gradient to the east and south of the repository. The contour interval in the lower figure is $0.2 \mathrm{~m} . \ldots \ldots \ldots . \ldots 144$

Figure 5-5: Particle pathlines from the water table below the repository to the five $\mathrm{km}$ boundary. . . . . . . . . . . . . . . . . . . 147

Figure 5-6: Contour map of median groundwater travel time from below the repository to the five $\mathrm{km}$ boundary. Also shown is a plot of distributions of travel time at three locations, corresponding to the locations $\mathrm{A}, \mathrm{B}$, and $\mathrm{C}$ on the contour map. The shaded areas indicate regions where the particle tracking method failed for some particles. The grid points on the upper figure indicate the locations of particle release. . . . . . . . . . . . . . . . . . . . 148

Figure 5-7: Contour map showing the variability of groundwater travel time from the water table below the repository to the five $\mathrm{km}$ boundary. The non-parametric statistic used to represent variability is the normalized interdecile range. The outline of the repository is shown. The shaded areas indicate regions where the particle tracking method failed for some particles. The grid points on the figure indicate the locations of particle release. . . . . . . . . . . . . . . . 149

Figure 5-8: Contour map showing the percentage of particles with travel times of less than 1,000 years to the five $\mathrm{km}$ boundary. Outline of the repository is shown. The shaded areas indicate regions where particle tracking method failed for some particles. The grid points on the figure indicate the locations of particle release. . . . . . . . . . . . . . . . 151

Figure 6-1a: Combined unsaturated and saturated zone particle travel times along cross-section AA. . . . . . . . . . . . . . . . . . . 155

Figure 6-1b: Combined unsaturated and saturated zone particle travel times along cross-section CC. . . . . . . . . . . . . . . . . . . 156

Figure 6-2: Locations within the potential repository where cumulative probability functions were determined for groundwater travel times from the repository horizon to the accessible environment. . . . . . . . . . . . . . 157 
Figure 6-3: CDF's of combined unsaturated-zone saturated-zone particle travel times for particle released in the southern (A) and eastern (B) portions of the potential repository footprint (see Figure $6-2$ for locations). . . . . . . . . 158

Figure 6-4: $\quad C D F$ 's of combined unsaturated-zone saturated-zone particle travel times for particle released in the northern $(A)$, western $(B)$ and central $(C)$ portions of the potential repository footprint (see Figure $6-2$ for locations). . . . . . . . . 159 


\section{List of Tables}

Table 2-1: Hydrogeologic stratigraphy at the Yucca Mountain site. . . . . . . . . 7

Table 2-2: Occurrences of perched water in boreholes (refer to Figure 3-1 for borehole locations). . . . . . . . . . . . . . . . . 10

Table 3-1: Description of porosity in the nine hydrogeological units. . . . . . . . . 22

Table 3-2: $\quad$ Matrix porosity conditioning data sources (Refer to Figure 3-1 for borehole locations). . . . . . . . . . . . . . . . 28

Table 3-3: Regression relationships between porosity and Ksat for zeolitic and non-zeolitic units (see Figure 3-6). . . . . . . . . . . . . . . . . . 29

Table 3-4: $\quad$ Summary of available van Genuchten parameter data. . . . . . . . . . . 32

Table 3-5: Fracture frequency data sources. . . . . . . . . . . . . . 39

Table 3-6: Mean fracture frequency and the probability of hitting a 10 foot interval without a fracture for each hydrogeological unit. . . . . . . . . . . . . 40

Table 3-7: Expected fracture aperture of each hydrogeological unit (Wilson et al., 1994). . 43

Table 3-8: $\quad$ Parameters derived from air permeability data. . . . . . . . . . . . . . 46

Table 3-9: Reported ranges of spatial correlation. Spherical models were used in all studies. . . . . . . . . . . . . . . . . . . . . 49

Table 3-10: Discretization of cross-sections for geostatistical simulation. . . . . . . . . 54

Table 3-11: Summary of derivation of parameters at the flow model scale. . . . . . . 68

Table 3-12: Porosity means and standard deviations for the hydrogeological units as extracted from porosity realization number one of cross-section AA. It was not possible to extract a representative sample from unit \#5 due to its limited extent in cross-section AA. For comparison, the measured porosity means and standard deviations shown in Table $3-1$ are repeated here. . . . . . . . . . . . . . . . . . .69

Table 4-1: Number of elements in each computational grid. . . . . . . . . . 81

Table 4-2: Mean fracture frequency and the probability of hitting a 10 foot interval without a fracture for each hydrogeological unit used for sensitivity study. . . . . . 115

Table 4-3: Total and fractional amount of water flowing through the fractures at four locations as a result of the one-week infiltration pulse.. . . . . . . . . 132

Table 5-1: Hydraulic parameters used in the calibrated flow model. . . . . . . . . 142

Table 5-2: Comparison of simulated and measured heads for the calibrated flow model. . 142 


\section{Acronyms}

CDF Cumulative Distribution Function

CFR Code of Federal Regulations

$\mathrm{CH} \quad$ Calico Hills Unit

DOE Department of Energy

DKM Dual Permeability Model

ECM Equivalent Continuum Model

ESF Experimental Studies Facility

FEP Features, Events, and Processes

GLINTMOD GSLIB-Lynx Integration Module

GWTT Groundwater Travel Time

GWTT-94 Groundwater Travel Ttime, 1994 study

GWTT-95 Groundwater Travel Ttime 1995 study

NRC Nuclear Regulatory Commission

PA Performance Aassessment

PTn Paintbrush Tuff Non-welded Unit

QA Quality Assurance

SGSIM Sequential Gaussian Simulator

STAFF3D Solute Transport and Fracture Flow in 3 Dimensions

SZ

Saturated zone

$\mathrm{TCW}$

Tiva Canyon welded unit

TOUGH2 Transport of Unsaturated Groundwater and Heat

TSPA Total Systems Performance Assessment

TSw Topopah Spring Welded Unit

USGS United States Geological Survey

$\mathrm{UZ}$

Unsaturated Zone

YMP

Yucca Mountain Project 


\section{Executive Summary}

\section{Introduction}

This report describes the analyses performed to model groundwater flow at the potential high-level nuclear waste repository site at Yucca Mountain, Nevada and to provide estimations of pre-waste-emplacement groundwater travel time (GWTT-95). These analyses include calculations of groundwater flow in the unsaturated zone above and below the potential repository, as well as flow in the saturated zone to the five $\mathrm{km}$ limit of the accessible environment. Although the regulatory criteria concerning groundwater travel time are no longer being used as measures of subsystem performance, the underlying analyses of fast-path groundwater flow are integral to any safety assessment of the potential site. These analyses will be incorporated into future repository performance assessment calculations.

Because groundwater flow is expected to be one of the most important mechanisms for the transport of radionuclides from the repository, the characterization of groundwater flow is of great importance in an evaluation of the suitability of the site. The US Nuclear Regulatory Commission is responsible for licensing any nuclear-waste repository and has established groundwater travel time as one of the subsystem regulations for the assessment of repository performance. This performance requirement states that "...pre-waste-emplacement groundwater travel time along the fastest path of likely radionuclide travel from the disturbed zone to the accessible environment shall be at least 1,000 years...." In addition to the Nuclear Regulatory Commission subsystem performance requirement, the Department of Energy has stated a similar disqualifying condition that was originally intended to be used as a site-selection criterion.

This study includes several enhancements relative to previous analyses of groundwater travel time at the Yucca Mountain site. More sophisticated geostatistical simulation and upscaling methods have been applied to the characterization of material properties in the numerical modeling domain of the unsaturated zone. Recently acquired data on hydrologic parameters have been incorporated into the modeling effort. The conceptual model of groundwater flow processes in the unsaturated zone has been generalized to include the possibility of disequilibrium, fast-path flow in fractures. This more general conceptual model of flow is implemented using the dualpermeability model (DKM), which is generally recognized as more physically realistic than the previously employed equivalent continuum model (ECM). Numerical modeling of groundwater flow and an analysis of the resulting travel times in the saturated zone are also included in this report.

\section{Conceptual Model}

The conceptual model of flow processes, geologic conditions, and boundary conditions at the Yucca Mountain site forms the basis for numerical modeling of groundwater flow and analysis of groundwater travel time. This conceptual model is based on current understanding of flow in fractured media under unsaturated and saturated conditions and on geologic and hydrologic data 
from the site. Because site-specific data are sparse, the conceptual model encompasses a range of possible flow conditions.

The geology of the site is observed to consist of a series of gently-dipping volcanic strata, that alternate from welded to non-welded tuffs. The site is divided into major structural blocks by several north-striking, west-dipping normal faults and more poorly delineated northwest trending structures. Bedrock fracture frequency is higher in fault zones and in more densely welded intervals of the volcanic strata.

Meteorological data and observations of the shallow subsurface indicate that infiltration of groundwater is highly variable in both time and space. Recent estimates of the spatial distribution of average infiltration are based on the conclusion that infiltration is generally higher on ridge crests and side slopes and is generally lower in washes. These estimates have been incorporated as surface boundary conditions in this study. Perched water has been encountered within the unsaturated zone (UZ) in several boreholes, indicating the variability of unsaturated flow at the site. The configuration of the water table indicates that saturated flow at the site is generally northwest to southeast, with considerable variation in the magnitude of the hydraulic gradient.

Conceptual models of flow processes in fractured media have evolved as site characterization at Yucca Mountain has proceeded. Initial models of unsaturated flow assumed that flow occurs primarily in the rock matrix at slow velocities, with fractures forming capillary barriers between matrix blocks. Alternatively, fractures have been proposed as locations of relatively focused, fast-path groundwater flow in the unsaturated zone. A more general conceptualization is that unsaturated flow occurs in both fractures and matrix, interacting in response to local hydrologic conditions and media characteristics. Two alternative numerical implementations of conceptual models that incorporate fracture and matrix flow are the equivalent continuum model and the dual-permeability model. Though computationally simpler, the equivalent continuum model contains the inherent assumption that capillary pressures are the same in the matrix and fractures at any given location in the system. The dual-permeability model allows disequilibrium in capillary pressure between matrix and fractures, which is a more general conceptualization of flow processes that permits the propagation of faster flow through fractures under conditions of lower matrix saturation and over greater distances.

The conceptual model used in this study for the unsaturated zone is consistent with the emphasis placed on the propagation of fast-path flow in fractures, which may be initiated by infiltration at the surface or within locally saturated zones (perched water). The dual-permeability model is used and steady-state conditions are assumed for numerical modeling of groundwater flow along four cross-sections. Surface infiltration rates are specified and the water table is used as the lower boundary. Heterogeneous material properties for both the fracture and matrix domains are specified, thus incorporating site data, an interpretive geologic model, and uncertainty in the spatial distributions of hydrologic parameters.

In contrast, the conceptual model of the flow in the saturated zone (SZ) used in this study consists of a deterministic representation of the geology of the upper portion of the saturated zone. Variability of flow in the SZ is incorporated through the simulation of hydrodynamic dispersion in the particle tracking method. The equivalent porous medium conceptual model of flow, a confined system, and steady-state conditions are assumed. The flow system is recognized 
to be dominated by flow through the fractures. The lateral boundaries of the saturated zone model are defined as specified head and are taken, in a general sense, from the results of regional-scale flow modeling.

\section{Parameter and Geologic Model Development}

Numerical modeling of groundwater flow for analyses performed in this study use hydrogeologic parameters that have been developed from available site data. Models of rock properties along cross-sections in the unsaturated zone are constrained by borehole data, a deterministic geologic framework model, and regression relationships among various parameters. Because of uncertainty in hydrologic parameters, a probabilistic approach in the unsaturated zone has been adopted in this study. Ten independent realizations of the material properties in the numerical model domain are generated along each cross-section using geostatistical simulation techniques.

The primary matrix parameters of porosity and saturated hydraulic conductivity are simulated by sequential Gaussian simulation and coregionalization. Facture frequency is also simulated by sequential Gaussian simulation. The geologic framework model of the unsaturated zone is used to constrain, but not strictly define, material properties during the geostatistical simulation process using a newly developed technique. Matrix moisture characteristic curve parameters and fracture porosity are determined from regression relationships with saturated hydraulic conductivity and matrix porosity. Zones of enhanced fracture frequency associated with tectonic activity are simulated using indicator simulation techniques. Hydrologic parameters which are simulated on the finer geostatistical scale are upscaled to the coarser flow-model scale using power-law averaging.

Material properties are deterministically assigned to hydrogeologic units in the numerical flow model of the saturated zone. The geologic framework model of the saturated zone is based on a published interpretation of the geology at the water table.

\section{Numerical Flow Modeling in the Unsaturated Zone}

The conceptual model of groundwater flow in the unsaturated zone is implemented for analysis of groundwater travel time using numerical methods. Solution of steady-state, unsaturated flow is obtained by the TOUGH2 code using the integral finite-difference method. Boundary conditions consist of specified liquid flux at the upper boundary, no-flow along the lateral boundaries, and specified capillary pressure at the water table along the lower boundary. The numerical grid for each cross-section is adapted to conform to the dipping strata, the location of faults, and to the location of presumably hydrologically significant units such as the nonwelded Paintbrush Tuff interval and the basal vitrophyre of the welded Topopah Spring Tuff.

Flow modeling results indicate a generally favorable comparison between simulated matrix saturation and values of matrix saturation measured in core from boreholes near the crosssections. Simulated matrix saturations vary significantly among geostatistical realizations, but are generally lower in the nonwelded Paintbrush Tuff interval and under surface washes. Simulated 
matrix saturations are generally quite high in vitrophyres and in zeolitized regions. Flow velocities in both the matrix and fractures are generally vertical downward, with no apparent lateral diversion at unit interfaces. Simulated flow velocities in fractures exceed flow velocities in the corresponding matrix over most of the cross-sections. Groundwater flow from the matrix to the fractures only occurs in a few areas in the lower parts of the sections.

A particle tracking algorithm is employed to simulate the movement of water particles in the steady-state flow field calculated by the flow model. Water particles are tracked subject to advective transport, mechanical dispersion, and molecular diffusion. Advection occurs at the faster of the matrix and fracture flow velocities. Results indicate that particle movement is generally downward and there is variation in travel time among geostatistical realizations by approximately one to three orders of magnitude for any given location. Travel times from the repository horizon to the water table vary from approximately 50 years to greater than $1,000,000$ years for all realizations along the four cross-sections. Although most particle travel times exceed 1,000 years, a significant number of particles have travel times of less than 1,000 years.

Sensitivity analyses of the numerical flow model were performed to evaluate the importance of uncertainty in hydrologic parameters, of numerical modeling methods, and of alternative conceptual models of flow processes. A single representative realization of one crosssection was used for sensitivity analyses. Findings of the sensitivity analyses are summarized by the following points:

- Particle travel times are very sensitive to infiltration rates, though higher infiltration rates result in unrealistically high simulated matrix saturation within most of the section.

- Flow modeling results are sensitive to the degree of connectivity between the fracture and matrix continua. Further reduction of the fracture-matrix connectivity can offset the effects of increased infiltration on matrix saturation, resulting in very short ( $<10$ years) travel times.

- Reduction of fracture frequency results in short travel times, but does not favorably match observed matrix saturation values.

- Removal of the zeolitic region from the flow model results in somewhat longer travel times.

- Refinement of the numerical grid results in greater local variability in simulated matrix saturation and lower fracture saturation. Travel times are increased by approximately one to two orders of magnitude using the refined grid.

- Use of the equivalent continuum model results in a favorable match with observed matrix saturations, but much longer travel times through the section.

- The use of the van Genuchten moisture characteristic curve parameters from previous unsaturated flow modeling studies results in significant lateral diversion of flow in the PTn unit. The presence or absence of lateral diversion in flow modeling results thus appears to be very sensitive to these parameters. 
- The application of a relatively large, but realistic infiltration flux at the upper boundary of the flow model for a short time period (one week) results in the rapid propagation of increased fracture flow velocity within the mountain. The simulated pulse of increased fracture flow reaches the repository horizon within ten years of its initiation at the surface.

\section{Numerical Flow Modeling in the Saturated Zone}

The numerical flow model of the saturated zone is a three-dimensional domain extending beyond the limits of the controlled area of the Yucca Mountain site and consisting of five layers. Hydrostratigraphic units are defined primarily on the basis of degree of welding. The lateral boundaries are specified hydraulic head and the water table and lower boundary are specified as no-flow. Solution of the steady-state flow model is performed by the finite-element method using the STAFF3D code and a numerical grid consisting of 13,800 nodes.

The saturated zone flow model is calibrated based on measurements of hydraulic head in 27 wells and on the inferred direction and magnitude of the hydraulic gradient in the region downgradient from the repository. Reasonably accurate calibration is achieved for most wells by the inclusion of the Solitario Canyon fault, Solitario Canyon fault splay, Drill Hole Wash -structure, and the Yucca Wash structure as low hydraulic conductivity zones. The generally southeast-trending, low hydraulic gradient in the area to the south and east of the repository is simulated by the flow model.

A particle tracking algorithm that simulates the advective and dispersive transport of water particles in the saturated zone is used to determine travel times from the water table beneath the potential repository to the five $\mathrm{km}$ limit of the controlled area. Complete diffusive mixing between fractures and rock matrix is assumed in the particle tracking method. Groundwater flow in the region downgradient of the repository in the flow model is controlled by the geometry of the hydrostratigraphic units and the contrasts in hydraulic conductivity among the units. Results indicate that the simulated travel times from below the repository to the five $\mathrm{km}$ limit are very sensitive to the distance traversed by the pathline through the low-conductivity Calico Hills Formation. The distributions of particle travel times vary dramatically depending on the location where the particles are released beneath the repository. In general, particles that are released below the eastern part of the repository have long travel times $(>10,000$ years) and particles released below the western and southern parts of the repository have shorter travel times $(\sim 250$ to a few thousand years). The distributions of travel times for particles released beneath the central part of the repository are very broad, ranging from a few hundreds to a few tens of thousands years.

\section{Summary and Conclusions}

Numerical modeling of groundwater flow is used to determine the travel time from the potential repository to the five $\mathrm{km}$ boundary of the accessible environment and to assess the impacts of our uncertainty in conceptual models and hydrologic parameters. These calculations are performed separately for the unsaturated zone and saturated zone. The important findings of 
this numerical study for each of these zones are presented below. In addition, estimates of the combined unsaturated-zone and saturated-zone groundwater travel time distributions are made. Finally, recommendations based on these findings are presented.

\section{Unsaturated Zone:}

- Simulation of material properties using a method linking geostatistical simulation techniques to a deterministic geologic framework model incorporates parameter uncertainty and retains geologic realism.

- Numerically simulated values of matrix saturation compare favorably with observations made from boreholes.

- Flow modeling indicates that groundwater flow in both the matrix and fractures is vertically downward, with flow velocities in the fracture domain generally exceeding those in the matrix.

- Simulated particle travel times from the repository to the water table for any location along the cross-sections vary by about one to three orders of magnitude among geostatistical realizations, indicating the importance of heterogeneity and uncertainty in hydrologic parameters.

- Variations in particle travel times along cross-sections are due primarily to variations in the infiltration rate at the surface boundary and to the thickness of the PTn unit. Shorter travel times are associated with areas of higher infiltration and with regions of thinner PTn.

- Most simulated particle travel times from the repository to the water table exceed 1,000 years in the UZ. Some particle travel times less than 1,000 years occur at the western portion of cross-section $\mathrm{AA}$, the eastern and central portion of cross-section $\mathrm{BB}$, along cross-section $\mathrm{CC}$, and at the southeast end of cross-section $\mathrm{DD}$.

- Sensitivity analyses indicate that simulated particle travel times decrease in response to increased infiltration flux, decreased fracture-matrix connectivity, and decreased fracture frequency.

- It is possible to match observed matrix saturation profiles by inversely adjusting the infiltration and fracture-matrix connectivity in unison. This relationship and the difficulty of constraining these parameters make it difficult to assess the absolute values of travel time by model calibration to observed matrix saturation.

- Both the equivalent continuum and dual permeability models are able to match measured core saturations. Use of the ECM, however, results in significantly longer travel times.

- Although lateral flow is not simulated in the PTn unit by the DKM, it is simulated using the ECM and van Genuchten $\alpha$ parameter used in previous studies (Klavetter and Peters, 1986). 
- The introduction of a large transient pulse of infiltration along the upper boundary of the UZ flow model caused simulated flow velocities in the fracture domain to increase throughout the mountain, though the relative increase in velocity decreases with depth. This pulse of increased velocity reaches the repository horizon within ten years of the initiation of the pulse.

\section{Saturated Zone:}

- The SZ flow model is calibrated to measurements of hydraulic head at 27 wells and to the inferred direction and magnitude of the hydraulic gradient. Reasonable calibration is achieved by including zones of lower hydraulic conductivity along the Solitario Canyon fault, Solitario Canyon fault splay, Drill Hole Wash structure, and Yucca Wash structure.

- Simulated particle travel times from the water table below the potential repository to the five $\mathrm{km}$ boundary of the accessible environment are sensitive to the geometry of the hydrostratigraphic units, specifically the low-conductivity Calico Hills Formation. Pathlines through this unit result in travel times up to two-orders of magnitude longer than for pathlines through other units.

- In general, particles released at the water table below the eastern portion of the repository have travel times greater than 10,000 years, whereas many particles released below the southern and western portions of the repository have travel times less than 1,000 years.

\section{Combined UZ and SZ Travel Times:}

The distributions of groundwater travel time are estimated at several locations by combining travel times in the UZ and SZ for the designated base case. Total travel time distributions are determined by randomly summing travel times from a region along one of the UZ cross-sections with travel times from the water table below this region. This procedure implicitly assumes that fast pathways in the UZ do not necessarily coincide with fast pathways in the SZ. Of the five representative regions evaluated in this way, one distribution contains travel times of less than 1,000 years. In the southern part of the repository about $40 \%$ of the combined simulated particle travel times are less than 1,000 years.

Comparison of these results to the NRC and DOE GWTT regulations indicates that a significant portion of simulated total travel times (40\%) from the southern portion of the potential repository are less than the regulatory 1,000 year limit. However, interpretation of these results should include consideration of the parameter sensitivities, uncertainties, and assumptions in the underlying numerical modeling analyses. Sensitivity analyses indicate that particle travel times may vary several orders of magnitude in the UZ flow simulations within plausible ranges of some input parameters, such as infiltration flux and fracture-matrix connectivity. The conservative approximation used to track the movement of particles between the fractures and matrix emphasizes the fastest flow paths, but does not calculate a complete distribution of travel times in the UZ. There are important uncertainties in this analysis of GWTT, especially in the UZ, that preclude definitive evaluation of regulatory compliance. 


\section{Conclusions:}

The results of this study support the assumptions made regarding the importance of the conceptual model of flow processes and the incorporation of heterogeneity in material properties into the analyses. The more physically realistic DKM simulates the observed distributions of matrix saturations and simulates relatively faster groundwater flow in the fracture domain. The inclusion of uncertainty and heterogeneity in material properties in the UZ flow simulations results in considerable variability in simulated groundwater travel times. Simulations of groundwater flow are particularly sensitive the infiltration flux at the ground surface and to poorly constrained physical parameters describing the interaction between unsaturated groundwater flow in fractures and the matrix.

Recommendations based on the findings of this study are made with the intent of improving the assessment of groundwater travel time in particular and understanding of groundwater flow at the Yucca Mountain site in general.

\section{Recommendations:}

- Additional site-characterization data from the area of the southern portion of the potential repository are needed to assess groundwater travel times in this region. Findings from this study indicate the potential for relatively fast-path flow in both the UZ and SZ in this area, yet few borehole data are available.

- Infiltration and fracture-matrix connectivity are key modeling parameters that should be constrained as fully as possible through laboratory, field, and numerical experiments. Simulated groundwater travel times and matrix saturation are found to be sensitive to several parameters, but infiltration and fracture-matrix connectivity are critical to more accurate numerical flow modeling in the UZ.

- Field studies to obtain direct evidence of lateral flow in the PTn (or lack thereof) and laboratory work to better constrain the van Genuchten $\alpha$ parameter in the PTn are needed to validate numerical flow modeling in the UZ. The base-case results of this study did not show significant lateral diversion of groundwater flow associated with the PTn, but this result is shown to be sensitive to the conceptual flow model employed and the value of the van Genuchten $\alpha$ parameter used for matrix moisture characteristic curves. Analysis of possible lateral flow in the PTn is critical to evaluating groundwater flux at the potential repository horizon.

- Although the relative groundwater travel times in the SZ have been established in this study, the absolute values of travel times can only be accurately determined by acquiring additional data on hydraulic conductivity by field testing. Furthermore, the assumption of groundwater exchange between fractures and matrix must be substantiated by tracer experiments in the field. 


\section{CHAPTER 1 \\ Introduction}

This report describes the analyses performed in FY 1995 to model groundwater flow at the potential high-level nuclear-waste repository site at Yucca Mountain, Nevada. The work is part of a program intended to provide inputs to the Department of Energy's (DOE) site viability evaluations. To support DOE's addressing of these regulations, this work has calculated estimates of the travel time of a "particle" of water assuming no disturbance to the flow regime due to the presence of the nuclear waste. The analyses represent preliminary estimations of the groundwater travel time (GWTT), as defined in the Nuclear Regulatory Commission (NRC) subsystem regulations (described in Section 1.1 below). Since the completion of this work, the strategy of the Yucca Mountain Site Characterization Project (YMSCP) has changed to de-emphasize activities directed toward evaluating the subsystem performance requirements. This report presents results that were intended to support the groundwater travel time regulatory evaluation. Although the regulatory criteria that form the basis for the GWTT requirements are no longer being used as measures of subsystem performance, the underlying analyses are integral to any safety assessment of the potential site. Therefore, these analyses will be incorporated into future performance assessment calculations.

The GWTT-95 analyses provide an initial estimate of our interpretation of groundwater flow at the Yucca Mountain using a reasonable balance between sufficient detail in the modeled processes and a site-scale extent for the model domain. The GWTT-95 methodology is applicable not only to analyses in support of GWTT regulations, but also for other PA efforts, including the total-system performance assessments planned for the DOE's-Viability Assessment.

\subsection{Regulatory Issues}

Groundwater travel time is one of the subsystem regulations promulgated by the NRC for underground nuclear-waste repositories. The intent of such regulations is to provide additional means for the regulators to judge their confidence in the repository total-system performance requirements. Evaluation of GWTT is intended to provide an independent estimate of the ability of the potential repository site to isolate waste by defining a minimum time period for groundwater to move from the general area of the repository to the accessible environment.

The NRC GWTT Performance Requirement is given in 10 CFR 60.113(a)(2) (NRC, 1983) and the DOE GWTT Disqualifying Condition is contained in the DOE Postclosure Geohydrology guideline in 10 CFR 960.4-2-1 (d) (DOE, 1984). The Performance Requirement states that "...prewaste-emplacement groundwater travel time along the fastest path of likely radionuclide travel from the disturbed zone to the accessible environment shall be at least 1,000 years...." The disturbed zone has been defined by the NRC to include "...that portion of the controlled area the physical or chemical properties of which have changed as a result of underground ... construction 
or heat generated ... such that the resultant change of properties may have a significant effect on the performance of the geologic repository."

In addition to the NRC subsystem performance requirement, the Department of Energy has stated a similar performance condition that was originally intended to be used as a site-selection criterion. The Disqualifying Condition is that "A site shall be disqualified if the pre-wasteemplacement groundwater travel time from the disturbed zone to the accessible environment is expected to be less than 1,000 years along any pathway of likely and significant radionuclide travel."

\subsection{NRC Technical Exchanges}

The concept of a "disturbed zone" in GWTT calculations is something of a conundrum because the definition involves post-waste-emplacement effects (such as heat) although the GWTT calculation itself is for pre-waste-emplacement (i.e., ambient-temperature) conditions. This apparent inconsistency has led the NRC and DOE technical staffs to propose an alternative concept at technical exchange meetings. Under the alternative, pre-waste-emplacement GWTT calculations and post-waste-emplacement calculations would both be made starting from the location of the potential repository (instead of starting from the ambiguous disturbed zone). The two classes of distributions would be compared, and if the post-emplacement ones were not significantly faster than the pre-emplacement distributions, then the effects of disturbance by the repository (and thus the extent of the disturbed zone) could be considered negligible. If postemplacement GWTT distributions were faster than the pre-emplacement ones, then both the preand post-emplacement calculations would be redone starting at successively greater distances from the repository volume until the differences between the two classes of distributions became negligible.

The second area of discussion at the technical exchanges is the definition of what constitutes "faster" or "slower" GWTT distributions. The regulations, stated above, leave considerable room for interpretation of what could constitute the "... fastest path". Interpretations of the fastest path range from the absolute fastest travel time for any element in any GWTT distribution to a probabilistic prediction of the fraction of the distributions that are less than the regulatory limit. Because the definition was left unresolved in the regulations, it is anticipated that the NRC will make an interpretation in the future. Future GWTT investigations will explore the implications of the various alternatives.

\subsection{Overview of the GWTT-95 Study}

Based on the technical exchanges, the groundwater travel time investigations conducted in fiscal year 1995 (called GWTT-95) are intended to provide the pre-waste-emplacement GWTT distributions against which future post-waste-emplacement distributions can be compared. Because of the 1,000-year regulatory standard for travel time, the emphasis of the analyses is on modeling fast groundwater-flow paths in both the unsaturated zone (UZ) of Yucca Mountain and 
in the saturated tuffs (the saturated zone - SZ) beneath the mountain from the potential repository horizon to the accessible environment. The goal of these analyses is to estimate a combined (UZ and SZ) pre-waste-emplacement groundwater travel time.

The DOE approach to addressing both the NRC and DOE regulations for GWTT is based on evaluating the likelihood and significance of distributions of travel times. Thus, GWTT has been probabilistically calculated from the area of the potential repository in the unsaturated portion of Yucca Mountain and through the saturated tuffs to the accessible environment (defined as being located $5 \mathrm{~km}$ away from the repository).

The important issues defining the scope of this analysis are as follows: The analyses are considered to be "pre-waste-emplacement" because thermal perturbations arising from decay heat from the radioactive waste are not considered. The rock at the Yucca Mountain site, like many other natural geologic formations, has great variability in its physical and hydrologic properties. To reflect this in the modeling, the model hydrologic and physical parameters are represented as heterogeneous spatial distributions. The fastest flow of groundwater in the UZ is expected to occur in rock fractures, rather than through the rock matrix. To model these fast-path flow processes, two conceptual models have been investigated - the equivalent-continuum model (ECM) and the dual-permeability model (DKM).

Pre-waste-emplacement GWTT distributions are calculated by incorporating the following components: identification of features, events and processes (FEPs) comprising the scenarios being modeled, development of the UZ and SZ model domains, simulation of hydrologic model parameters in the UZ and SZ, calculation of flow in the UZ and SZ, estimation of travel times for water particles on the UZ and SZ flow fields, and combination of the UZ and SZ distributions. Simulations are made from the potential repository horizon in the $U Z$ to $5 \mathrm{~km}$ down-gradient from the repository in the SZ to produce an estimate of groundwater travel time to the accessible environment.

The FEPs modeled in the GWTT-95 work are consistent with the scenarios developed for nominal-flow conditions at Yucca Mountain. The nominal-flow scenarios are described in Barr and Hunter (1995) ${ }^{1}$.

The GWTT-95 model domains are built upon numerous prior analyses. These analyses have investigated the importance of heterogeneity in the physical and hydraulic properties of the unsaturated rock as a method to simulate the spatially and temporally variable groundwater flow behavior. The effects of heterogeneity in the unsaturated zone on flow have been developed by Robey (1994) on an INTRAVAL problem, and in the GWTT-94 analyses (Arnold et al., 1995). Both of these efforts simulated heterogeneous hydrologic properties on 2-dimensional crosssections with variations in the properties occurring over a scale of a few meters. The INTRAVAL work was intended to emphasize simulation of the heterogeneous properties; flow was modeled using a flow code (the DUAL code - Robey (1994)) that preserved flow variations resulting from

1. Barr, G. E. and R. L. Hunter. (1995). Reference Hydrologic Scenarios Developed from the Nominal Flow Report. Sandia Letter Report. Albuquerque, NM: Sandia National Laboratories. 
the heterogeneities. The GWTT-94 work continued the investigation of heterogeneities using the DUAL code. Heterogeneity has been implemented in the model by geostatistical simulation techniques. For both the INTRAVAL and GWTT-94 analyses, the geostatistical simulations produced realizations of the stratified hydrogeologic rock units. Further statistical simulations produced spatially variable values of porosity on the two-dimensional cross-sections. From these heterogeneous porosity fields the other hydrologic parameters (e.g., hydraulic conductivity and water-retention parameters) were then simulated from regression relationships.

The approach used to generate the UZ hydrogeologic model for GWTT-95 is an enhancement of the GWTT-94 procedures. In contrast to prior work, where the rock types and the hydrogeologic properties of those rock types were separately modeled using three-dimensional geostatistical techniques, in GWTT-95 materials properties are simulated in two dimensions using both measured borehole data and interpretive data from a three-dimensional geologic framework model. In GWTT-94, the boundaries between the rock-type strata were also statistically generated, resulting in some modeling artifacts that took the form of interfingering of adjacent units. To more realistically model the geologic regime, the geologic framework model is used to constrain the boundaries. The resulting two-dimensional cross-sections used for the GWTT-95 analyses appear to be layered, but the layers are derived from the materials properties simulations constrained by the deterministic model, and are still heterogeneous.

The parameter set used for flow modeling is an enhancement of the Total-System Performance Assessment (TSPA)-93 (Wilson et al., 1994) and GWTT-94 data sets (Schenker et al., 1995). Particularly for fracture hydrologic properties, the parameter data sets have been expanded with recently developed data.

In past flow modeling efforts the ECM has been used. With this model, the relationship between pressure head and hydraulic conductivity can be represented by a single curve that describes both matrix and fracture flow. Because the ECM assumes that fracture and matrix capillary pressures are always in equilibrium, fracture flow only occurs when the matrix is nearly saturated. Although the heterogeneous hydrologic-properties distributions produce regions of localized saturation, this restriction on fracture flow in the ECM may limit its realism in terms of the propagation of flow in fractures. The predecessor to the DKM, the dual-porosity model, was developed for modeling petroleum-production (Warren and Root, 1963). It has in the past only been applied to groundwater-flow problems on a limited basis. Like the dual-porosity model, the DKM uses two continua, one for the matrix and one for the fractures, in which the flows can be independently modeled. The two continua are linked by a coupling factor that permits transfer of water from the matrix to the fractures and back. This model has the potential to simulate fracture flow over a wider range of groundwater infiltration and saturation conditions, and may more realistically model conditions at Yucca Mountain.

For GWTT-95, groundwater flow in the UZ was modeled using both the ECM and DKM. Most analyses assumed steady-state boundary conditions, and flow simulations were run to steadystate conditions. In addition, flow was also modeled for some episodic boundary conditions. Four 2-dimensional transects were modeled on which the model parameters were stochastically 
assigned. Several realizations of the simulated hydrologic properties were modeled for each transect.

Because neither the INTRAVAL nor the GWTT-94 work included flow in the saturated zone, the GWTT-95 investigations referred to the work done in TSPA-93 (Wilson et al., 1994). For that analysis, a 3-dimensional deterministic geologic model of the saturated zone was constructed. The GWTT-95 SZ model is an enhancement of the TSPA-93 model. The SZ model covers an area of $172 \mathrm{~km}^{2}$ (extending from the potential repository to the 5-km boundary of the accessible environment) to a depth of $250 \mathrm{~m}$ below the water table. More of the available site data have been incorporated in the definition and layout of the stratigraphic units. The contacts for dipping units within the model are more accurately modeled than previously, and the hydrologic features of the faults have been incorporated.

Saturated-zone flow is modeled in 3-dimensions assuming flow through an equivalent porous medium of rock matrix and fractures. A single realization of the hydrologic parameters is used. The flow field is calculated using homogeneous intra-unit hydrologic parameters.

Groundwater travel time distributions are estimated by modeling the transport of "water particles" on four two-dimensional transects located in various parts of the Yucca Mountain site. Travel times are estimated by tracking the paths of water particles launched from the potential repository horizon. Depending on whether velocities are greater in the fracture or matrix domain at a given element, the particles can either travel by flow in the fracture or matrix domains. Depending on the proportion of fracture to matrix flow, different locations can have differences in the travel times in the unsaturated zone. The particles are assumed to be transported in the UZ and SZ flow fields primarily by advection in the fractures and/or matrix. In the UZ, particle flow paths are modified by diffusion and dispersion; in the SZ, longitudinal and transverse mechanical dispersion are applied to the particle trajectories to provide a distribution of transport paths. Particle tracking computer codes trace the paths of water particles in the UZ and SZ flow fields to determine distributions of travel times.

In addition to baseline analyses, sensitivity studies have been done to investigate the two conceptual models and the effects of varying the model domains and parameter distributions. Sensitivity analysis provides an assessment of the uncertainty of the calculations relative to the flow modeling assumptions. 
This page intentionally left blank. 


\section{CHAPTER 2 Conceptual Models}

The conceptual model of a hydrogeologic system, such as that at the Yucca Mountain site, provides a basis for numerical modeling of groundwater flow and the analysis of groundwater travel time. A conceptual model includes assumptions regarding the geologic configuration of the system, the relevant hydrologic boundary conditions, and potential groundwater flow processes for fractured porous media under conditions of the site. Because site-specific data are sparse, the conceptual model should encompass a range of possible flow conditions.

This chapter includes a brief description of observations of geologic and hydrologic conditions and some general inferences drawn from these observations regarding the flow system. Also included is an evaluation of alternative numerical methods for implementing the inferred conceptual models of flow. In conclusion, the specific conceptual models of flow in the unsaturated and saturated zones for the GWTT-95 analysis are presented.

\subsection{Yucca Mountain Site Hydrogeologic System}

A general overview of the geologic and hydrologic conditions is given below. For a more detailed description of observations at the site refer to Arnold et al. (1995) and Wilson et al. (1994).

\subsubsection{Geology}

Rock units in the region of Yucca Mountain include Paleozoic sedimentary and metasedimentary rocks, some Mesozoic intrusive igneous rocks, Cenozoic silicic and basaltic volcanic sequences, and alluvial deposits. At the site itself, the basement consists of Paleozoic carbonate rocks. A thick sequence of Miocene-age silicic ash-flow and air-fall tuffs erupted from the Timber Mountain-Oasis Valley caldera complex constitute the bedrock of the unsaturated and shallow saturated zones. Formation-level stratigraphic units roughly correspond to major volcanic eruptive events (see Table 2-1). The approximately tabular bodies of alternating welded and nonwelded tuffs may vary somewhat in thickness across the site, but generally have a high degree of lateral continuity (e.g., Istok et al., 1994). The major welded ash-flow tuffs are usually separated by thinner intervals of nonwelded or partially welded ash flows, air-fall tuffs, and reworked tuffaceous sediments. The potential repository is located in the TSw unit. The water table occurs in the Calico Hills Formation, Prow Pass Formation, and Bullfrog Formations in the area directly below the repository. Note that the stratigraphy presented in Table 2-1 is intended as a general guide to the volcanic section of the site. For unsaturated zone modeling a more detailed subdivision of the stratigraphic units is employed (see section 3.1.1). 
Alluvial deposits of varying thicknesses cover the bedrock surface over most of the site. Alluvium tends to be thickest in washes and channel bottoms, thinner on side-slope terraces, and very thin to absent on steep side slopes and ridge tops.

Table 2-1: Hydrogeologic stratigraphy at the Yucca Mountain site.

\begin{tabular}{|c|c|c|}
\hline Unit ID & Unit Name & Description \\
\hline $\mathrm{TCw}$ & Tiva Canyon welded & $\begin{array}{l}\text { Moderately to densely welded, devitrified ash-flow tuffs } \\
\text { belonging to the Tiva Canyon Tuff of the Paintbrush Group. }\end{array}$ \\
\hline PTn & $\begin{array}{l}\text { Paintbrush nonwelded } \\
\text { interval }\end{array}$ & $\begin{array}{l}\text { Nonwelded to partially welded, vitric and locally devitrified } \\
\text { tuffs belonging to the lowermost Tiva Canyon Tuff, Yucca } \\
\text { Mountain Tuff, Pah Canyon Tuff and uppermost Topopah } \\
\text { Spring Tuff of the Paintbrush Group; also includes air-fall } \\
\text { tuffs, "bedded tuffs", and intercalated reworked tuffaceous } \\
\text { sediments. }\end{array}$ \\
\hline TSw & Topopah Spring Welded & $\begin{array}{l}\text { Moderately to densely welded, devitrified ash-flow tuffs } \\
\text { including the upper vitrophyre belonging to the Topopah } \\
\text { Spring Tuff of the Paintbrush Group. }\end{array}$ \\
\hline TSbv & $\begin{array}{l}\text { Topopah Spring basal } \\
\text { vitrophyre }\end{array}$ & $\begin{array}{l}\text { Densely welded basal vitrophyre belonging to the Topopah } \\
\text { Spring Tuff of the Paintbrush Group. }\end{array}$ \\
\hline CHnv & Calico Hills vitric & $\begin{array}{l}\text { Lowermost nonwelded to partially welded part of the } \\
\text { Topopah Spring Tuff lying underneath the basal vitrophyre } \\
\text { and nonwelded to partially welded, vitric and locally argillic } \\
\text { ash-flow, bedded, and reworked tuffs of the tuffaceous beds } \\
\text { of the Calico Hills Formation. }\end{array}$ \\
\hline CHnz & Calico Hills zeolitic & $\begin{array}{l}\text { Nonwelded and partially welded zeolitic tuffs of the tuf- } \\
\text { faceous beds of the Calico Hills Formation and the bedded } \\
\text { tuffs overlying the Prow Pass Tuff. }\end{array}$ \\
\hline PP & Prow Pass & $\begin{array}{l}\text { Nonwelded, partially and moderately welded ash-flow tuffs } \\
\text { belonging to the Prow Pass Tuff of the Crater Flat Group. }\end{array}$ \\
\hline $\mathrm{BF}$ & Bullfrog & $\begin{array}{l}\text { Nonwelded, partially and moderately welded ash-flow tuffs } \\
\text { belonging to the Bullfrog Tuff of the Crater Flat Group. }\end{array}$ \\
\hline Tram & Tram & $\begin{array}{l}\text { Moderately to densely welded ash-flow tuffs belonging to } \\
\text { the Tram Tuff of the Crater Flat Group. }\end{array}$ \\
\hline
\end{tabular}

The volcanic tuffs dip approximately $5^{\circ}$ to $10^{\circ}$ to the east and have been offset by a series of north-striking, west-dipping normal faults (see Figure 2-1). The main repository block is bordered on the west by the Solitario Canyon fault and on the east by the Bow Ridge fault. A more poorly delineated, northwest trending fault, the Drill Hole Wash structure, extends along the northern boundary of the potential repository. The Ghost Dance fault extends in a north-south direction within the repository block. Fracturing of tuff units appears to be related to both tectonic processes and cooling processes. Fracture frequency is higher in fault zones such as the Ghost Dance fault, creating tectonic zones of cross-formational continuity within the fracture 
network. Cooling fractures form intra-formationally continuous fracture networks. Fracture frequency is generally higher in welded units, decreasing dramatically in air-fall tuffs and other non-welded units.

West

East

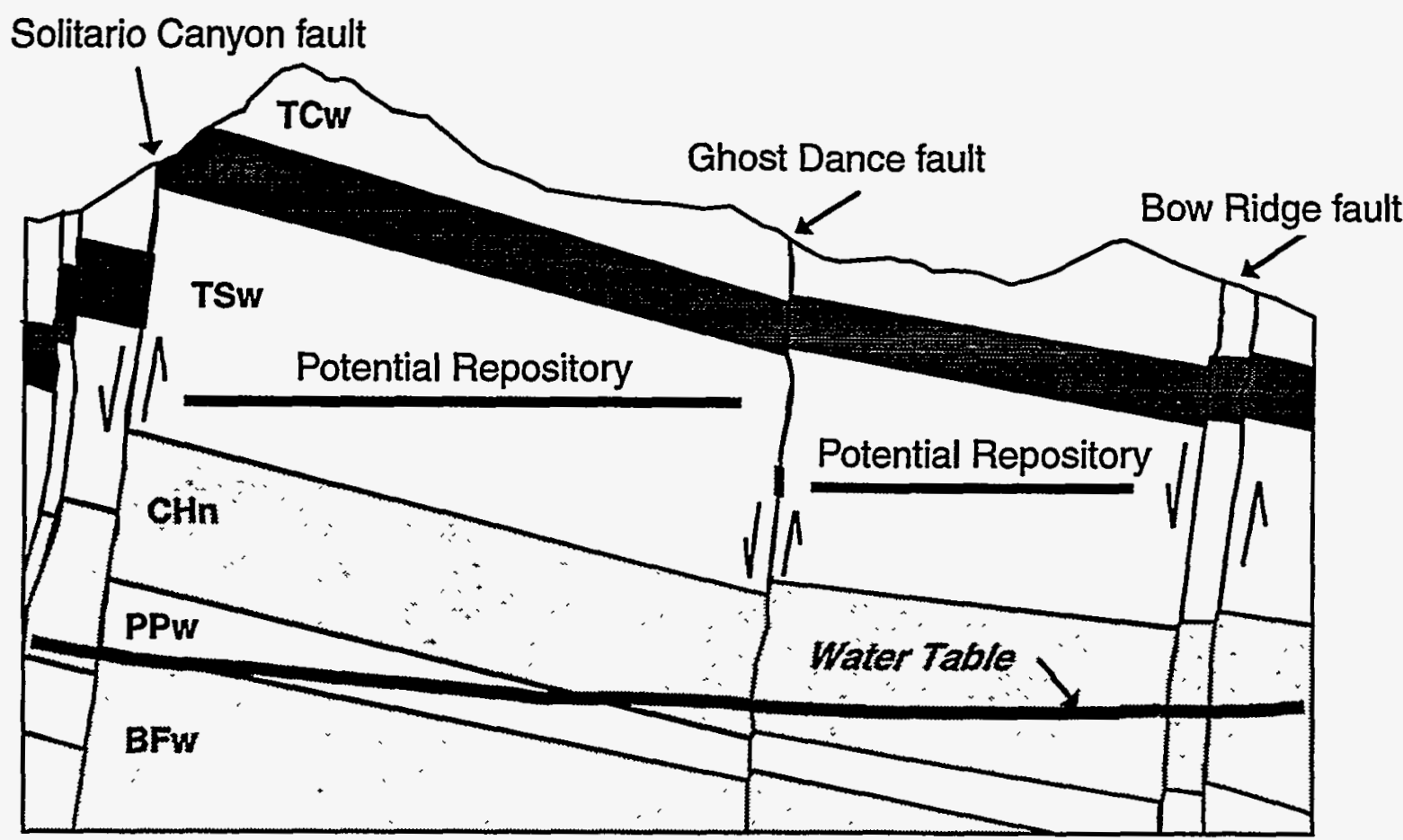

Figure 2-1 Schematic cross-section of the potential Yucca Mountain repository region.

\subsubsection{Surface Hydrology}

The average annual precipitation in the vicinity of the repository has been estimated as 17 cm (Hevesi et al., 1992). A majority of the precipitation occurs during low-intensity winter storms, generally occurring from November to April. Summer monsoon storms tend to be shorter duration, higher intensity precipitation events that occur in July and August (Hevesi and Flint, 1993). Accumulation of runoff in washes is a relatively rare event associated with more intense monsoonal storms.

Potential evapotranspiration greatly exceeds precipitation in the arid environment of Yucca Mountain. However, calculations of potential evapotranspiration do not account for the heterogeneous temporal and spatial distributions of precipitation and evapotranspiration processes. The depth to which evapotranspiration influences moisture content is unknown. Monitoring of moisture content in a set of 34 shallow boreholes using a neutron-backscatter moisture probe has been used to estimate shallow infiltration at a variety of different settings at the site (Flint et al., 1994). These transient moisture content data along with assumptions about 
the association of infiltration with geographic parameters have been used to estimate the spatial distribution of infiltration by Hudson and Flint ${ }^{2}$. An alternative estimation of the areal distribution of infiltration based on the Maxey-Eakin method has also been performed by Hevesi and Flint ${ }^{3}$. See section 4.1 of this report for a more detailed discussion of the results of Hudson and Flint ${ }^{2}$.

\subsubsection{Subsurface Hydrology}

\subsubsection{Unsaturated Zone}

Subsurface hydrology in the unsaturated zone (UZ) has been observed through the drilling of boreholes and examination of the hydrologic properties of the boring cores. Measurements of hydrologic parameters such as bulk density, porosity, particle density and in situ saturation, gravimetric water content, and saturated hydraulic conductivity have been made on core samples in most of the geologic units. In addition, testing in the boreholes, such as air-permeability tests of the fracture permeability (LeCain and Walker, 1994) and measurements of in situ temperature, pneumatic pressure and water potential (Kume and Rousseau, 1994) are being conducted. Additional hydrogeologic parameters have been measured along transects of outcrops (Flint et al., 1996; Istok et al., 1994). Measured matrix saturations vary from unit to unit, tending to be lower in the higher porosity units such as the PTn.

Perched water has been observed in several of the drillholes (Table 2-2). There is some question as to whether the perched water found in borings drilled in Drillhole Wash is natural or drilling fluid that was lost during the drilling of USW G-1. UZ-1 was drilled in 1983 and there is not as much documentation for the hole. Drilling stopped when the perched water was found at $387 \mathrm{~m}$. It is believed that this water was in the Calico Hills unit, but this is not certain. Perched water has also been found in the southern portion of the potential repository in boring SD-7 just west of the Ghost Dance fault.

\subsubsection{Saturated Zone}

Groundwater flow in the saturated zone under Yucca Mountain occurs in the Cenozoic volcanic rocks and the underlying Paleozoic sedimentary strata. The Paleozoic strata beneath Yucca Mountain are part of the regionally extensive carbonate aquifer of the south-central Great Basin (Winograd and Thordarson, 1975). At the present time, direct observation of the saturated zone is limited to 30 drill holes. Depth to the water table varies from approximately $275 \mathrm{~m}$ in valley floors to about $750 \mathrm{~m}$ at ridgetops in the vicinity of the site (Czarnecki and Luckey, 1989). The general configuration of the water table indicates groundwater flow from the northwest to the

2. Hudson, D. B. and A. L. Flint. 1996. Estimation of Shallow Infiltration and Presence of Potential Fast Pathways for Shallow Infiltration in the Yucca Mountain Area, Nevada. U.S. Department of Energy Technical Report, TIS\#960242.

3. Hevesi, J. A. and A. L. Flint. (in review). Geostatistical Model for Estimating Precipitation and Recharge for the Yucca Mountain Region, Nevada-California, 1993. Water-Resources Investigations Report. Denver, CO: US Geological Survey. 
Table 2-2: Occurrences of perched water in boreholes (refer to Figure 3-1 for borehole locations).

\begin{tabular}{|l|l|l|l|l|}
\hline Borehole & $\begin{array}{c}\text { Depth to } \\
\text { Perched } \\
\text { Water } \\
(\mathrm{m})\end{array}$ & $\begin{array}{c}\text { Elevation } \\
\text { of } \\
\text { Perched } \\
\text { Water }(\mathrm{m})\end{array}$ & \multicolumn{1}{|c|}{$\begin{array}{l}\text { Unit in which Perched water was } \\
\text { Encountered }\end{array}$} & $\begin{array}{c}\text { Polymer } \\
\text { Drilling } \\
\text { Fluid from } \\
\text { USW G-1? }\end{array}$ \\
\hline \hline UZ-14 & 384.6 & 956 & $\begin{array}{l}\text { Basal portion of the Topopah Spring Tuff } \\
\text { non-lithophysal unit. Water appeared to } \\
\text { be perched on clay layer developed on top } \\
\text { of the welded basal vitrophyre of the } \\
\text { Topopah. }\end{array}$ & traces \\
\hline NRG-7/7A & 460.2 & 822.1 & $\begin{array}{l}\text { Non-welded base of the Topopah Spring } \\
\text { Tuff. The water was perched on top of the } \\
\text { Calico Hills Tuff. }\end{array}$ & Yes \\
\hline SD-9 & 452.3 & 843.1 & $\begin{array}{l}\text { Calico Hills, 15 feet below the upper } \\
\text { contact of the Calico Hills with the } \\
\text { Topopah Spring Tuff. }\end{array}$ & Yes \\
\hline UZ-1 & 387 & 962.0 & Calico Hills? & Yes \\
\hline SD-7 & $479.8 *$ & 883.3 & $\begin{array}{l}\text { At the contact between the vitric and the } \\
\text { zeolitic Calico Hills }\end{array}$ & No \\
\hline * - perched water was first encountered 485.4 - 488.3 m below the ground surface and subsequently rose. \\
\hline
\end{tabular}

southeast at the site. The magnitude of the horizontal hydraulic gradient, as indicated by the potentiometric surface, varies dramatically across the site (Robison, 1984). Beneath most of the potential repository and to the south and east, the hydraulic gradient is very small (about 0.0001 ). Directly to the north of the repository, the water-table elevation increases by approximately 300 $\mathrm{m}$, defining a region with a large hydraulic gradient $(>0.15)$. Another significant step in the water table occurs to the west of the potential repository, where the horizontal hydraulic gradient is $>0.03$. Anomalously low heat flow measurements and variations in temperature at the water-table (Sass et al., 1988) indicate the possibility of significant vertical flow of groundwater in the SZ at the site.

\subsection{Conceptual Models of the Hydrogeologic System}

Inferences regarding the geologic and hydrologic conditions and processes at the Yucca Mountain site continue to evolve as additional data are acquired. New insights into the nature of groundwater flow in fractured media have continued to contribute to the conceptualization of flow processes in a generic sense. Analysis of fast-path flow in the UZ is particularly sensitive to the conceptualization of flow processes. 


\subsubsection{Geology}

Conceptual models of volcanic stratigraphy and structural geology have important implications for groundwater flow in both the UZ and SZ at the site. Stratigraphic classification of geologic units reflects the purpose of the classification exercise. In the study of groundwater flow, classification of strata on the basis of relevant hydrologic properties is required and may vary with the motivation of the analysis. Previous studies (e.g., Arnold et al., 1995; Wilson et al., 1994; Barnard et al., 1992) have employed differing sets of hydrogeologic units based on factors including welding, porosity, vitrification, and zeolitization. Limitations on the number of data and the ability to define parameter distributions for some strata preclude further subdivision of hydrostratigraphy in some cases.

Zeolitization is important to flow modeling at Yucca mountain because is causes a large reduction in the hydraulic conductivity relative to a small reduction in porosity. Zeolite minerals generally form in tuffs due to increased temperature and moisture. Therefore, zeolitization has mostly occurred closer to the water table in the Calico Hills Formation and the Prow Pass Tuff where the units were at one time saturated. Zeolites are also present, though not pervasively, in the PTn, indicating high moisture contents at one time. Zeolitization is more pervasive in the northern part of Yucca Mountain and is absent in locations to the south (USW GU-3 and Busted Butte) (Moyer and Geslin, 1995). Greater zeolitization to the north could be indicative of the water table position or the proximity to the caldera complex.

Structural interpretations of the geology at the site are based primarily on surface mapping and on limited geophysical data. Major faults have been delineated at the surface, but the degree of faulting by smaller-offset faults is uncertain, especially in regions such as the imbricate fault zone to the east of the Ghost Dance fault (Figure 2-1). There is additional uncertainty about the configuration of major faults at greater depth. The north-striking normal faults may be listric, converging with a low-angle normal fault at depths as shallow as $1500 \mathrm{~m}$ (Scott, 1990) or they may extend to depth at relatively high angles. Geophysical data have been used to hypothesize the existence of a buried graben, coincidental with the large hydraulic gradient in the saturated zone, in the area directly north of the repository (Fridrich et al., 1994).

\subsubsection{Unsaturated Zone}

\subsubsection{Infiltration}

Infiltration at the ground surface is accepted as the primary source of groundwater in the UZ flow system by all of the alternative conceptual models used in recent performance assessments. However, the magnitude and distribution of infiltration vary among different conceptual models. Montazer and Wilson (1986) believed that infiltration occurs episodically during large precipitation events and is focused in fractures in the TCw. They further proposed that runoff which is concentrated in washes leads to increased infiltration in these areas. Flint et al. (1994) asserted that infiltration is higher on sideslopes and ridge tops because of lower evapotranspiration due to the presence of clay soils and bedrock fractures, whereas infiltration is lower in active channels and terraces due to greater evapotranspiration of moisture stored in 
relatively thick alluvium. Because of differences in solar radiation and evapotranspiration between south-facing slopes and north-facing slopes, increased infiltration on north-facing slopes has been proposed. Variations in infiltration depending on outcropping bedrock unit were proposed by Flint and Flint (1994). Recent interpretations by and Hevesi and Flint ${ }^{4}$ have stressed the correlation between elevation and infiltration.

Shallow infiltration is a transient process due to seasonal variations in precipitation and the episodic nature of recharge events. The depth at which transient infiltration is "averaged" into net infiltration depends on the conceptualization of shallow infiltration processes and on the conceptual model of fracture-matrix flow interaction processes at greater depths. The depth to which evapotranspiration-induced flow will occur is unknown. The choice of a steady-state or transient conceptual model of flow is partially dependent on the conceptualization of infiltration processes.

\subsubsection{Flow Processes}

Groundwater flow in the UZ can occur as flow in the rock matrix and as flow in the fractures. The nature of the interaction of flow between matrix and fractures has important implications in the conceptualization of the flow system. The simplest conceptual model assumes that flow occurs exclusively in the matrix (Kaplan, 1993; Bloomsburg et al., 1989; Lin and Tierney, 1986); fractures do not participate in the flow process, but may act as capillary barriers to impede unsaturated flow. Conversely, another highly simplified representation of the system assumes that the most significant flow path is through the fractures that may occur as sparsely distributed "weeps" (Wilson et al., 1994; Barnard et al., 1992; Nitao and Buscheck, 1991). A more general conceptual model of the UZ flow process is that flow occurs in both the matrix and the fractures, being dominated by flow in either the matrix or the fractures depending on the local hydrologic conditions (Wang and Narasimahn, 1985, 1986, and 1988; Sinnock et al., 1984). In a locally saturated area there can be flow from the matrix to the fractures. The transient-flow scenario recognizes the episodic nature of infiltration events and includes the propagation of saturated flow from the surface in fractures. The significance of transient flow to the analysis of fast-flow paths in groundwater travel time depends on the depth to which saturated pulses penetrate before imbibition into the matrix.

Early conceptual models of unsaturated flow at the site were developed to visualize the large-scale flow system and the potential changes in the hydrology due to the presence of a repository. Montazer and Wilson (1986) believed that infiltration occurring during large precipitation events is focused in fractures in the TCw and diverted laterally by the PTn. Lateral flow continues until it reaches a fault, where the water moves downward. They speculated that perched water zones occur at these fault boundaries. Wang and Narasimhan's conceptual model had transient flow occurring primarily in fractures with dissipation of the transient pulse by the nonwelded Paintbrush tuff. In this model, flow is primarily vertical, with some lateral diversion of flow due to inclined hydrologic units (Wang and Narasimhan, 1985, 1986, 1988). In their model, Sinnock et al. (1984) assumed the unsaturated flow at the site is one-dimensional and

4. see footnote 3 , page 9 . 
transient. The influence of faults on the groundwater flow system is uncertain. However, conceptual models generally agree that they act to limit lateral movement of flow by behaving as barriers or drains.

The observations of perched water in boreholes at several locations and the occurrence of relatively young environmental tracers at depth ${ }^{5}$ have prompted reinterpretations of the conceptual models of flow to account for the heterogeneous nature of flow processes. These observations imply a complex groundwater flow system in which fractures support significant flow in some regions and matrix flow dominates in others. The resulting conceptual model places greater emphasis on the heterogeneity of the media that controls the unsaturated flow.

\subsubsection{Saturated Zone}

The conceptual model of groundwater flow processes in the SZ is that flow occurs predominantly in the fracture networks in both the volcanic units and in the Paleozoic carbonate aquifer. For groundwater flow on large scales, fracture connectivity is generally assumed to be high enough to justify a continuum representation of fracture permeability. The degree of interaction between flow in the fractures and matrix may influence groundwater travel times in the system.

Conceptual models of the saturated zone proposed by Czarnecki and Waddell (1984), Czarnecki (1985), Czarnecki (1989), Sinton (1989), and Fridrich et al. (1994) differ primarily in the processes controlling the large hydraulic gradient north of the site. The primary representations of the system are the diversionary and non-diversionary fault models (Fridrich et al., 1994). Fridrich et al. (1994) recommend identifying individual hydrostratigraphic units by the degree of welding, style of crystallization, fabric, secondary alteration, tectonic fracturing, and faulting. Their conceptual model predicts that zones of high hydraulic conductivity will exist in the densely welded tuffs and coarse-fabric tuffs, and parallel to faults. All the saturated zone conceptual models describe a three-dimensional flow system through a faulted, fractured porous medium.

\subsection{Alternative Implementations of Conceptual Flow Models}

\subsubsection{Overview of Conceptual Flow Models}

A limited number of numerical models are available to describe the various conceptual flow processes in fractured rock. These models, ranging from the equivalent porous medium model to the discrete fracture model, are currently at various stages of development and maturity. Figure 2-2 summarizes the major models that can be used to describe flow through fractured media. The models are presented along with a sketch of the model domain and the characteristic curves associated with each conceptualization.

5. Al Yang, personal communication, 1995. 
REPRESENTATION

Equivalent Single Continuum

EQUIVALENT

POROUS MEDIUM

COMPOSITE POROSITY

(EQUIVALENT CONTINUUM)

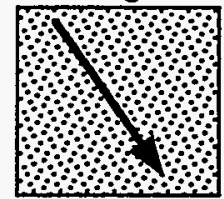

Equivalent Matrix

and Fracture Continuum

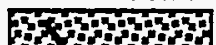

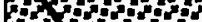
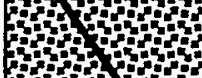

$x^{2}+y^{2}+3 y^{2}$
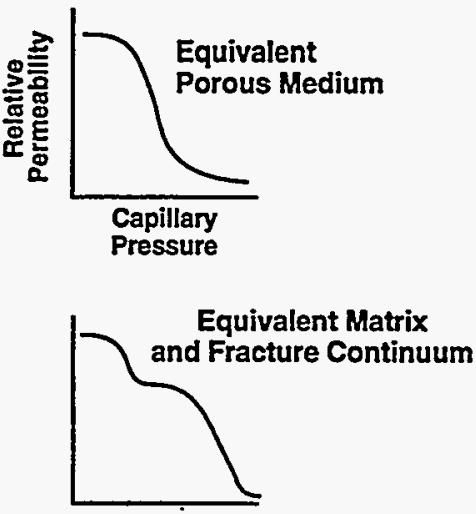

DUAL POROSTTY
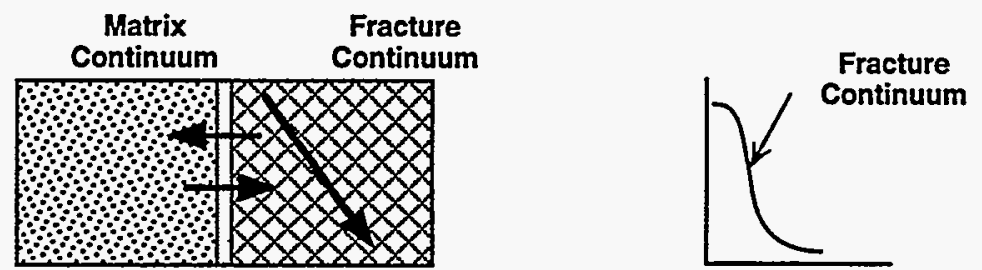

DUAL

PERMEABILITY
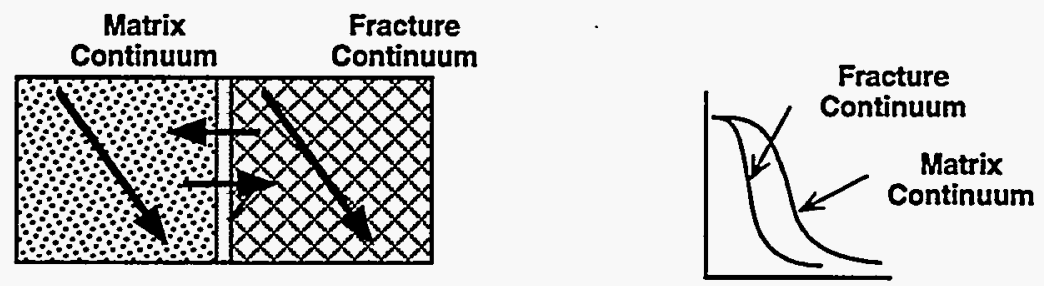

DISCRETE FRACTURES WITHOUT MATRIX
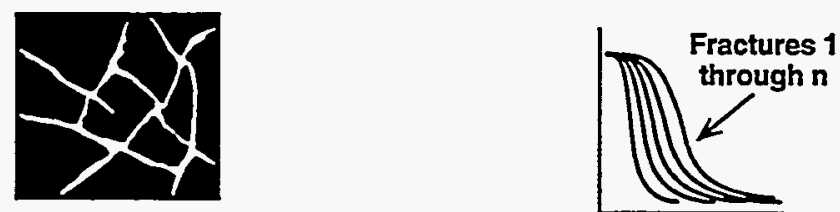

DISCRETE FRACTURES WITH MATRIX
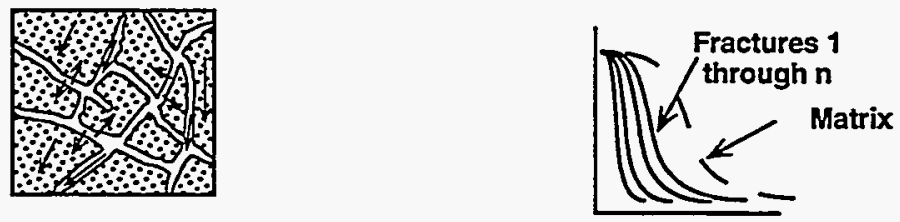

Figure 2-2 Alternative conceptual models and their corresponding relative permeability characteristic curves for flow through fractured rock. 
Of the models shown in Figure 2-2, several are not suitable for use in the UZ calculations in this study. The equivalent porous medium model, for example, represents the domain with either fractures or matrix materials, but not both. Since flow in the UZ will likely depend on the combined response of both domains, the equivalent porous medium model is not used in the UZ simulations. The dual-porosity model, which does not allow flow to be transmitted through the matrix domain, is also not used. Finally, while discrete fracture models have been applied to saturated domains, the implementation of these complex models to the UZ have not been fully developed. Therefore, the discrete fracture models are excluded from this study. A more thorough description of the remaining two models, the equivalent continuum model (ECM) and the dualpermeability model (DKM), is provided in the remainder of this section.

\subsubsection{Comparison of the ECM and DKM Conceptual Models}

The ECM has been used extensively in describing flow through fractured rock as a result of its relative simplicity and ease of computational implementation (Arnold et al., 1995; Robey, 1994; Dudley et al., 1988). In this model, the capillary pressures in the matrix and fractures are assumed equal. As a result, the flow through this fracture-matrix system is equivalent to flow through a composite porous medium that has hydraulic properties comprised of both fracture and matrix properties. Dudley et al. (1988) express that for conditions similar to those found at Yucca Mountain (i.e., low infiltration rates and good coupling between the fracture and matrix), the equivalent continuum model provides a reasonable approximation to fracture-matrix flow.

However, recent studies have shown that flow processes such as fingering in fractures (Glass and Tidwell, 1991) and mechanical aspects such as fracture coatings may effectively reduce the coupling between the fractures and matrix. This may cause pressure disequilibrium between the fractures and matrix, even under low infiltration rates.

If pressure equilibrium cannot be assumed, more elaborate models such as the DKM must be used. Unlike the ECM, the DKM represents the fractures and matrix as separate continua. As a result, different pressures can exist in the fractures and matrix, which allows flow to occur between the two continua. Propagation of flow in fractures is more likely to be observed in these models, depending on parameters such as the fracture-matrix connection area and the capillary pressure gradient that affect the coupling of flow between the fracture and matrix continua. However, the additional flexibility and capabilities of the DKM are complicated by the additional parameter uncertainties and computational efforts associated with simulating flow in separate fracture and matrix domains. As a result, several analyses have been performed recently to compare the ECM and DKM under various hydrologic scenarios and to determine the feasibility of implementing the DKM in the highly heterogeneous environments proposed in this study.

Ho et al. (1995) performed a series of benchmarks to illuminate the strengths, weaknesses, caveats, and capabilities of the ECM and DKM. One- and two-dimensional simulations were performed to investigate important issues such as heterogeneities, fracture-matrix coupling, and episodic events associated with groundwater flow in the UZ. Results showed that the ECM and DKM produced similar results in saturation profiles and fluxes when the infiltration rates were low $(\sim 1 \mathrm{~mm} / \mathrm{year})$ and the coupling between the fracture and matrix elements was good. However, when the infiltration rate was large or when the fracture-matrix coupling was reduced, the DKM produced a significant amount of flow through the fractures, which was more 
reasonable and conceptually consistent than the results of the ECM, which showed minimal or no fracture flow under most conditions. Similar differences were found by Eaton et al. (1996) in a three-dimensional model of an infiltration experiment at Fran Ridge. These discrepancies are of considerable importance when considering the possibility of episodic infiltration events. Fast groundwater flow paths through the unsaturated zone are likely to be initiated by episodic events that produce high infiltration rates for short periods of time. Only the DKM and the discrete fracture model are capable of simulating this type of behavior.

Another important issue raised by Ho et al. (1995) concerns the coupling between the fracture and matrix elements in the DKM. Flow between the fracture and matrix elements is treated similarly to flow between any two elements in the same continuum. Darcy's law governs the flux of liquid between the two elements where the liquid pressures can be different in each element. Figure 2-3 shows a sketch of a vertical, one-dimensional DKM with flow occurring in both the fracture and matrix continua as well as between the two continua. Of particular interest is the connection area between the fracture and matrix element shown in the formulation for fracture-matrix flow. The calculated connection area based on geometry assumes that the entire area between the fractures and matrix is available to flow. However, small scale processes such as fingering within a single fracture (Glass and Tidwell, 1991) and mechanical aspects such as fracture coatings (Thoma et al., 1990) may effectively reduce the conductance between the fractures and matrix. In addition, observations of flow in a fracture network have shown that only a fraction of the fractures are hydrologically active (Nicholl and Glass, 1995). As a result, the calculated connection area between the fractures and matrix may be significantly overestimated. If only a fraction of the fracture plane is wetted due to fingering, and only a fraction of the fractures in a fracture network are flowing, then the connection area between a fracture and matrix element can be effectively reduced by several orders of magnitude. This hypothetical reduction produced greater flow velocities in the fractures and slightly lower matrix saturations in Ho et al. (1995). As a conservative approximation, a reduction of three orders of magnitude was used as a base case for the DKM simulations in this study. More comprehensive derivations of the fracturematrix coupling terms can be found in Ho et al. (1995).

The results of Ho et al. (1995) showed that the application of the DKM to completely heterogeneous, two-dimensional domains was feasible. Steady-state and transient simulations were successfully performed and compared to the ECM results. Based on the conclusions and recommendations from that study, the DKM is used as the primary model for GWTT-95 UZ groundwater flow calculations.

Both the ECM and DKM are continuum models; fractures are not explicitly modeled, but are represented as a continuum that has flow properties characteristic of flow through fracture networks. A discrete-fracture model requires that individual fractures and their flow properties be enumerated in the model. Because of uncertainty in our knowledge of fracture network properties at Yucca Mountain and the large scale of the GWTT analyses, continuum models are probably satisfactory. It is possible that "dead-end" fractures could flow directly into the matrix of underlying units, especially in the case of a welded unit overlying a nonwelded unit. This type of "cross-continuum" flow is not represented in the DKM and could possibly influence the simulation of flow. 
fracture matrix

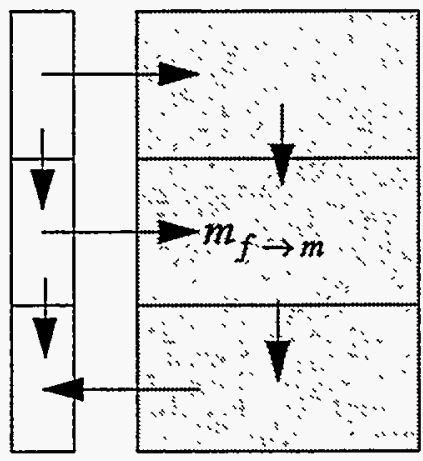

$m_{f \rightarrow m}=\rho A_{f-m} \frac{k_{m} k_{r m}}{\mu}\left(\frac{P_{c, m}-P_{c, f}}{d}\right)$

where

$\begin{aligned} & A_{f-m}= \text { effective connection area } \\ & \text { between fracture and matrix }\end{aligned}$

$d=$ effective distance between fracture and matrix

Figure 2-3 Mass flow between fracture and matrix elements in a dual-permeability model. The fracture-matrix conductance depends, in part, on the effective connection area, which may be reduced as a result of small scale processes such as fingering, preferential flow paths, and mechanical aspects such as fracture coatings. See Section 4.2.1 for definitions of other variables.

\subsection{Conceptual Models Employed in the GWTT-95 Analysis}

Numerical simulations utilized in this study are based on a conceptual model of the unsaturated zone which includes uncertainty in the spatial distribution of material properties within the mountain and allows for non-equilibrium flow of groundwater in both fractures and matrix. This is a considerably more general representation of unsaturated flow than was used in the GWTT-94 study (Arnold et al., 1995). The conceptual model of flow in the GWTT-95 analysis focuses on fast-path flow in fractures, which may be initiated by infiltration at the surface or within locally saturated zones (perched water) in the UZ. Propagation of groundwater flow in fractures may persist with depth through regions of unsaturated matrix due to limitations on fracture-matrix interaction. The emphasis of this study is on the propagation of flow in fractures, whereas the work of Arnold et al. (1995) focused on the initiation of fracture flow in zones of perched water.

The conceptual model of the saturated zone includes a single, deterministic representation of the geology (distribution of material properties) and an equivalent porous-medium representation of flow processes. Site-scale flow in the $\mathrm{SZ}$ is conceived to be controlled by contrasts in permeability among volcanic units and within fault zones in the shallow SZ. Greater certainty about the nature of flow processes in the SZ is offset by relatively fewer data than are available for the UZ. 


\subsubsection{Unsaturated Zone}

\subsubsection{Geology}

The physical geometry of the geologic system and hydrologic parameters are conceptualized to include both a deterministic and a random component. The deterministic component is supplied by a geologic framework model and the random component is approximated by stochastic simulation. The resulting bounded heterogeneity of both fractures and matrix exercises important control of the flow system by focusing groundwater flow and producing variations in the saturation of the medium. A two-dimensional representation of the system is employed because of the importance of lateral flow focusing processes. Multiple realizations of the system are used to encompass a reasonable range of uncertainty in the spatial distribution of material properties.

\subsubsection{Boundary Conditions}

The modeled boundary conditions of flow in the UZ are infiltration flux at the ground surface, specified pressure at the water table, and no-flow across lateral boundaries. Surface infiltration of groundwater is specified based on independent research aimed at quantifying this flux and determining the spatial and temporal distribution of infiltration (Hudson and Flint ${ }^{6}$; Hevesi and Flint ${ }^{7}$ ). Infiltration is conceptualized to occur primarily within fractures. The water table forms a natural boundary at which the liquid pressure is equal to the gas pressure. Lateral boundaries are located just beyond major bounding faults, which would be expected to intercept any lateral diversion of moisture flow.

\subsubsection{Flow Processes}

The dual permeability conceptual model of flow is utilized to describe flow in UZ for this study. Steady-state conditions are assumed to approximate the state of groundwater flow, although preliminary simulations of transient infiltration are included as a part of the sensitivity analysis (see section 4.5:9). The DKM explicitly accounts for flow in both the fracture and matrix continua in a coupled manner without requiring capillary pressure equilibrium between the two domains (see section 2.3). The conceptual model of flow employed in this study recognizes the probable limitations on coupling of flow between fractures and matrix due to restricted wetting of fracture surfaces. This conceptualization of flow processes in the UZ is consistent with the emphasis placed on simulating the propagation of fracture flow by this study.

6. see footnote 2 , page 9 .

7. see footnote 3 , page 9 . 


\subsubsection{Saturated Zone}

\subsubsection{Geology}

The conceptual model of geology of the saturated zone is based on a deterministic depiction of the upper portion of the SZ. This geologic representation is based on borehole data, projection of surface faults to depth, and interpretation of general structural and stratigraphic trends. Hydrostratigraphic units are distinguished principally on the basis of the proportion of welded intervals within each unit. Faults with apparent influence on the distribution of hydraulic head are included as separate, low-conductivity units.

\subsubsection{Boundary Conditions}

The lateral boundaries of the SZ are defined as specified hydraulic head. The influence of the lateral, specified-head boundary conditions is reduced near the center of the model in the sense that the simulated flow is redistributed by the hydrostratigraphic geometry in regions far from these boundaries. For example, the vertical hydraulic gradient at the lateral boundaries is zero, but there is significant vertical flow simulated in the central portion of the model because of this redistribution. The upper and lower boundaries are specified as zero flux. Infiltration undoubtedly crosses the water table in some areas of the model, but this flux is expected to be insignificant relative to the horizontal flow in the SZ (at least for average infiltration rates of less than a few tenths $\mathrm{mm} / \mathrm{year}$ ). The lower boundary is somewhat arbitrary; however, the upper 250 $\mathrm{m}$ of the tuff aquifer is assumed to be the most significant part of the flow system with regard to transport from beneath the repository to the $5 \mathrm{~km}$ limit. This assumption is consistent with the geometry of the geologic framework model (see Section 3.1.2) which indicates that groundwater may flow under units with low hydraulic conductivity, such as the Calico Hills unit, in the region downgradient of the repository within the upper $250 \mathrm{~m}$ of the SZ.

\subsubsection{Flow Processes}

The equivalent porous medium conceptual model of flow is used in the SZ flow modeling reported in this study. It is recognized that under saturated conditions the actual flow system will be dominated (in terms of flux) by flow through fractures. It is assumed that the fracture network is sufficiently dense and that fluid residence times are sufficiently long to allow the equivalent porous medium approximation to be used for the GWTT calculations. The flow in the saturated zone is assumed to be in steady state. 


\section{CHAPTER 3 \\ Parameter and Geological Model Development}

The 1995 GWTT calculations are carried out on four separate cross-sections through the Yucca Mountain site. Models of rock properties along these cross-sections are constrained by borehole data and by two-dimensional slices of a deterministic three-dimensional, geologic framework model of the Yucca Mountain area. The lithologic units within the geologic framework model are combined into nine hydrogeological units. The borehole information provides mean values and standard deviations of properties within each of the nine hydrogeological units as well as regression relations between properties. Matrix porosity and fracture frequency are geostatistically simulated and saturated hydraulic conductivity is derived from a model of linear coregionalization with porosity. The simulated properties are upscaled to the computational grid using power-law averaging. Values of properties that are not simulated, (e.g., van Genuchten parameters, fracture permeability), are derived at the computational grid-block scale through regression relations and conceptualizations of fracture openings as parallel plates. The rockproperty models are evaluated for their consistency with conceptual models of the geology at Yucca Mountain.

\subsection{Geologic Framework Models}

\subsubsection{Unsaturated Zone}

For the purposes of the GWTT-95 study, a geologic framework model is described as a three-dimensional model of lithology and faults (locations and offsets). Two geologic framework models have been employed in the GWTT-95 calculations. These are the preliminary version of the subregional model constructed by Zelinski and Clayton ${ }^{8}$ and the model constructed by Fridrich et al. (1994). Modeling work done in the unsaturated zone relies solely on the Zelinski model. The GWTT-95 unsaturated zone flow modeling has been completed on four separate cross-sections intersecting the area of the potential repository (Figure 3-1).

For the GWTT calculations, lithologic units are combined into hydrogeological units (see Table 3-1) based on similarities in porosity and geologic origin. This combining is done by 1) examining the porosity distributions between lithologic units, 2) considering the geologic genesis of the units and 3) considering the lateral extent and thickness of the units as represented in the digital geologic framework model. Thickness is important because of the way the framework model discretizes the geology and how that discretization is used by the geostatistical simulator. For each cross-section, the digital framework model is limited to discretizing the geology into $200 \times 200$ elements. These elements are on the order of $75 \mathrm{~m}$ long and $17 \mathrm{~m}$ high. The

8. Zelinski, W.P. and R.W. Clayton. (in review). A $3 D$ Geologic Framework and Integrated Site Model of Yucca Mountain: Version ISM1.0. Las Vegas, NV: Civilian Radioactive Waste Management System, Management \& Operating Contractor Report. 


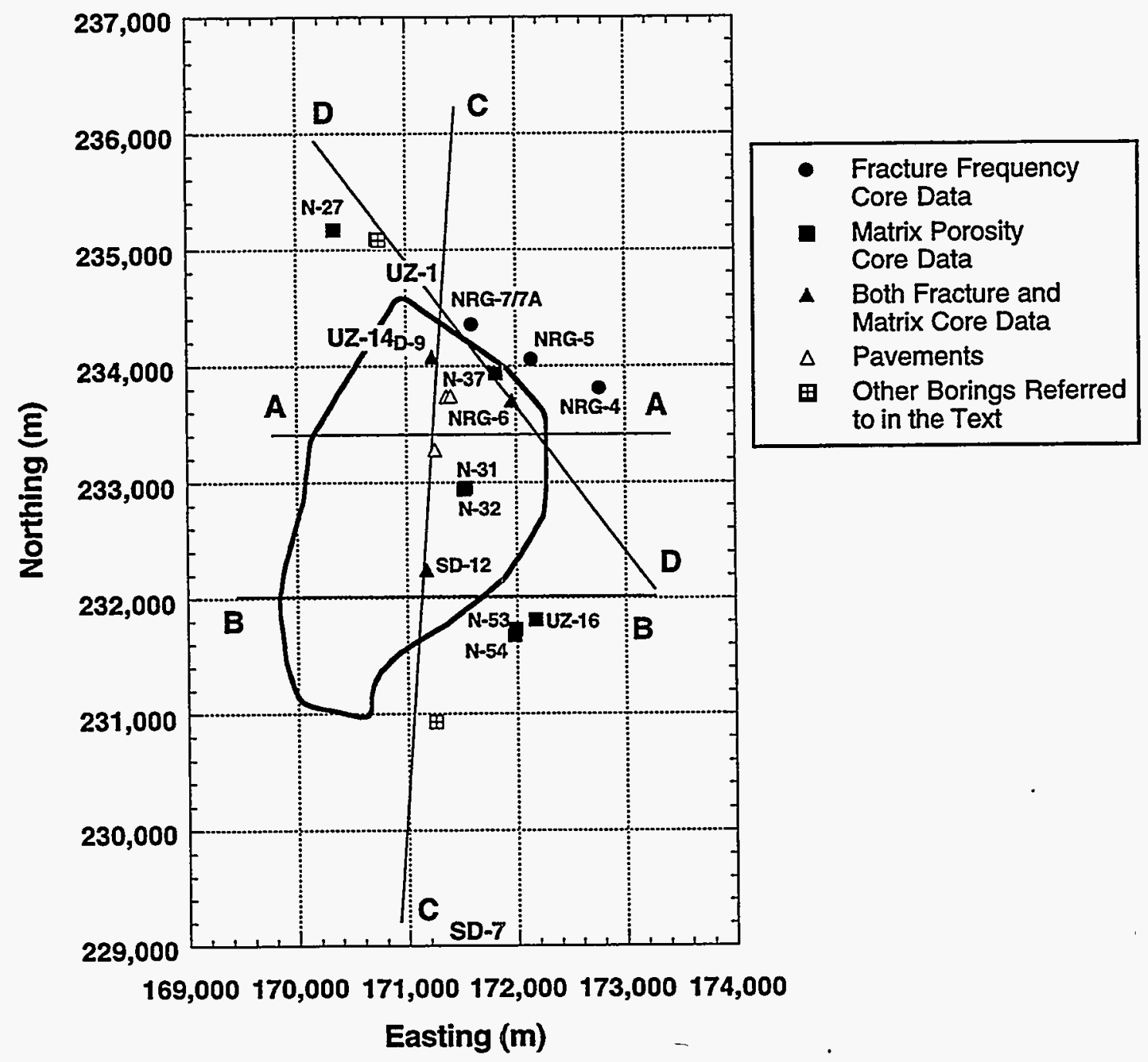

Figure 3-1 Map showing location of borings from which matrix porosity and/or fracture frequency conditioning data were determined. The polygon defining the outline of the potential repository is shown along with the GWTT-95 cross-sections.

discretization is done with regular, rectangular elements that do not adapt to the geology. This coarse discretization may lead to thin units being combined along with a thicker unit(s) into a single element. The percentage of each hydrogeological unit comprising each element is provided by the digital framework model. The link between the geostatistical simulator and the framework 
model is currently set up to query the framework model for the hydrogeological unit at the location being simulated. If there is more than one hydrogeological unit at a location, the geostatistical simulator currently retains the unit with the highest percentage (largest volume within the digital framework model element) at that location. Through this process, thin units may never be used in the geostatistical simulation and it is not necessary to keep them as separate hydrogeological units during the lumping. The link between the geostatistical simulator and the digital framework model is modified to retain thin, hydrologically important units if necessary. For this reason, thin, hydrologically significant units have been kept separate during the combining of lithologic units into hydrogeological units.

The porosity distributions for the nine hydrogeological units used in GWTT-95 modeling are shown in Figure 3-2. The mean and standard deviation of porosity for each hydrogeological unit are shown in Table 3-1.

Table 3-1: Description of porosity in the nine hydrogeological units.

\begin{tabular}{|c|c|c|c|c|}
\hline Hydrogeological Units & $\begin{array}{c}\text { Unit } \\
\text { Number }\end{array}$ & $\begin{array}{c}\text { Mean } \\
\text { Porosity }\end{array}$ & $\begin{array}{c}\text { Standard } \\
\text { Deviation }\end{array}$ & $\begin{array}{c}\text { Number of } \\
\text { Porosity } \\
\text { Msmts. }\end{array}$ \\
\hline \hline Vitrophyre & 1 & 0.05 & 0.05 & 75 \\
\hline Tiva Canyon Welded & 2 & 0.09 & 0.07 & 203 \\
\hline Topopah non-Lithophysal & 3 & 0.10 & 0.04 & 779 \\
\hline Topopah Lithophysal & 4 & 0.13 & 0.04 & 512 \\
\hline PP-BF welded & 5 & 0.15 & 0.04 & 21 \\
\hline Calico Hills & 6 & 0.23 & 0.07 & 181 \\
\hline PP-BF non-welded & 7 & 0.25 & 0.06 & 44 \\
\hline Paintbrush Tuff non-welded \#2 & 8 & 0.28 & 0.10 & 57 \\
\hline Paintbrush Tuff non-welded \#1 & 9 & 0.46 & 0.08 & 204 \\
\hline
\end{tabular}

Under guidance set forth by the Yucca Mountain Site Characterization Office (YMSCO), Performance Assessment analyses must be based on data qualified under the Quality Assurance program. For such "QAed" data, there is traceability of custody, consistent collection techniques and measurement calibrations. Much of the data collected in the past at Yucca Mountain may not meet all of the requirements for QA'ed data. If sufficient qualified data were available, these data were used in these analyses. In other cases, due to limited availability, "non-QAed" data were used. It should also be noted that data were being generated in parallel with the development of the GWTT-95 calculations. Therefore, data that were not used for part of these analyses were incorporated where possible when they became available. 


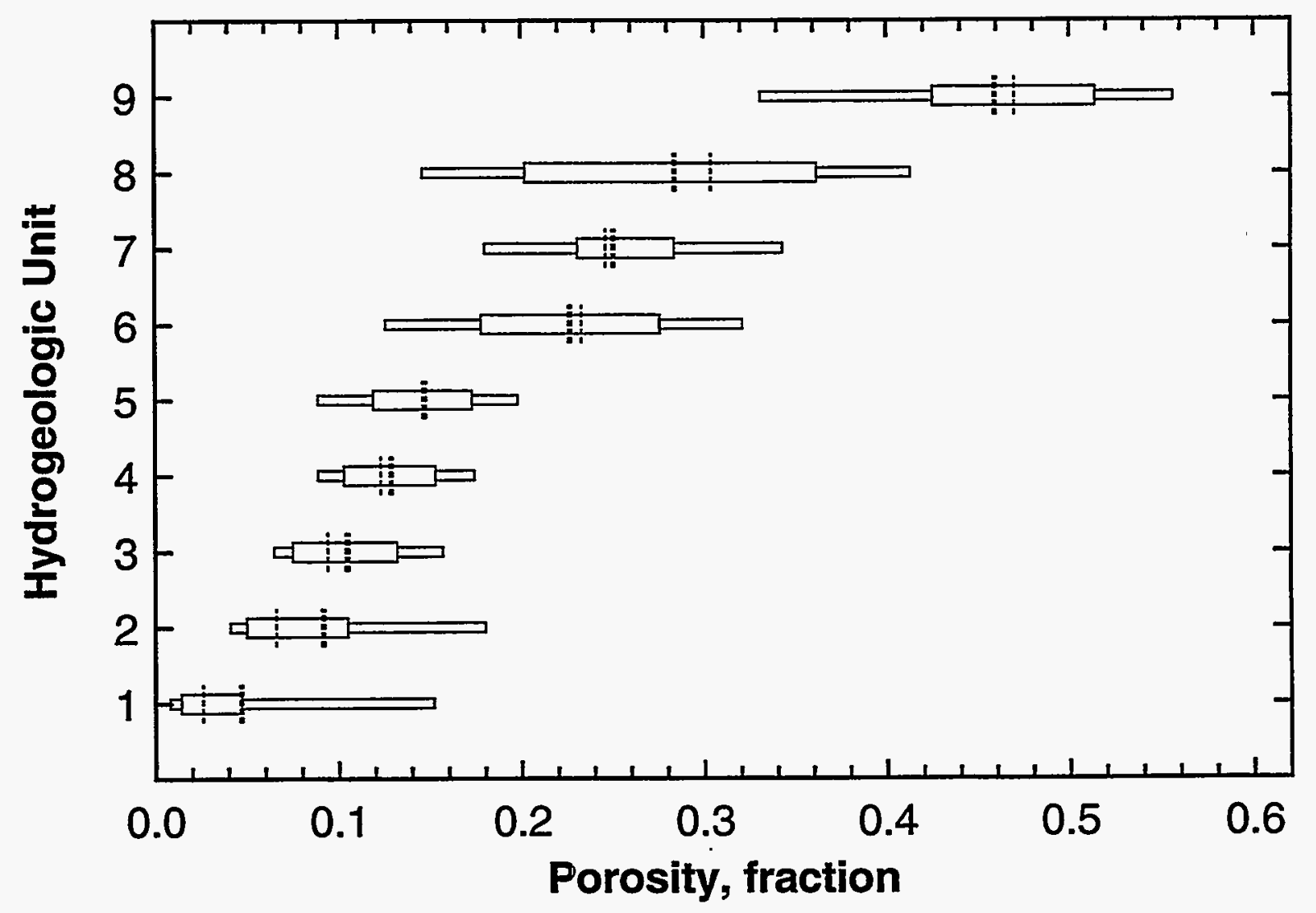

Figure 3-2 Distributions of porosity for each hydrogeological unit. The numbers refer to the numbers used in Table 3-1. The thick dashed lines indicate the mean of each distribution and the thin dashed lines indicate the median. The 25th and 75th percentiles are defined by the thick boxes and the 10th and 90th percentiles are defined by the thin boxes.

Measurements taken from the core of five borings were used to determine the distribution of porosity in the hydrogeological units: NRG-6, SD-9, SD-12, UZ-14 and UZ-16. The neutron holes were not used for these analyses because they generally only penetrate the TCw. By using only quality assured porosity data to define the hydrogeological units, the spatial distribution of the data is limited. For many of the lithologic units, there are no qualified porosity data. These units have been combined into their respective hydrogeological units based on the geologic similarity between them and the other stratigraphic units comprising the hydrogeological unit.

This combination of lithologic units results in nine hydrogeological units that have been used to constrain simulations of rock properties. The final hydrogeological unit may appear in several, non-contiguous, locations within the model domain (e.g., the TSw lithophysal unit). The hydrogeological units in the unsaturated zone are shown for the four cross-sections in Figures 3-3 and 3-4. 


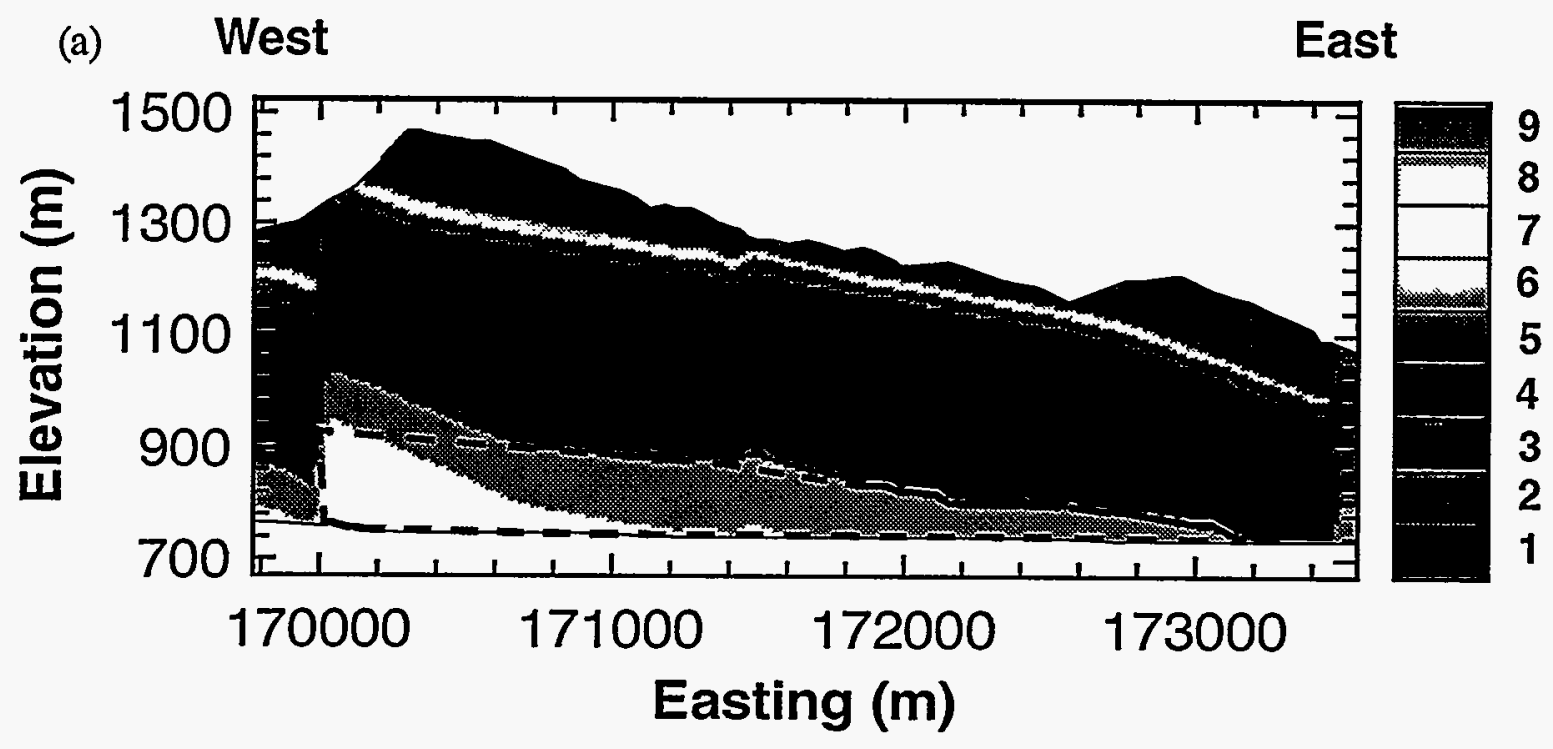

(b) West East

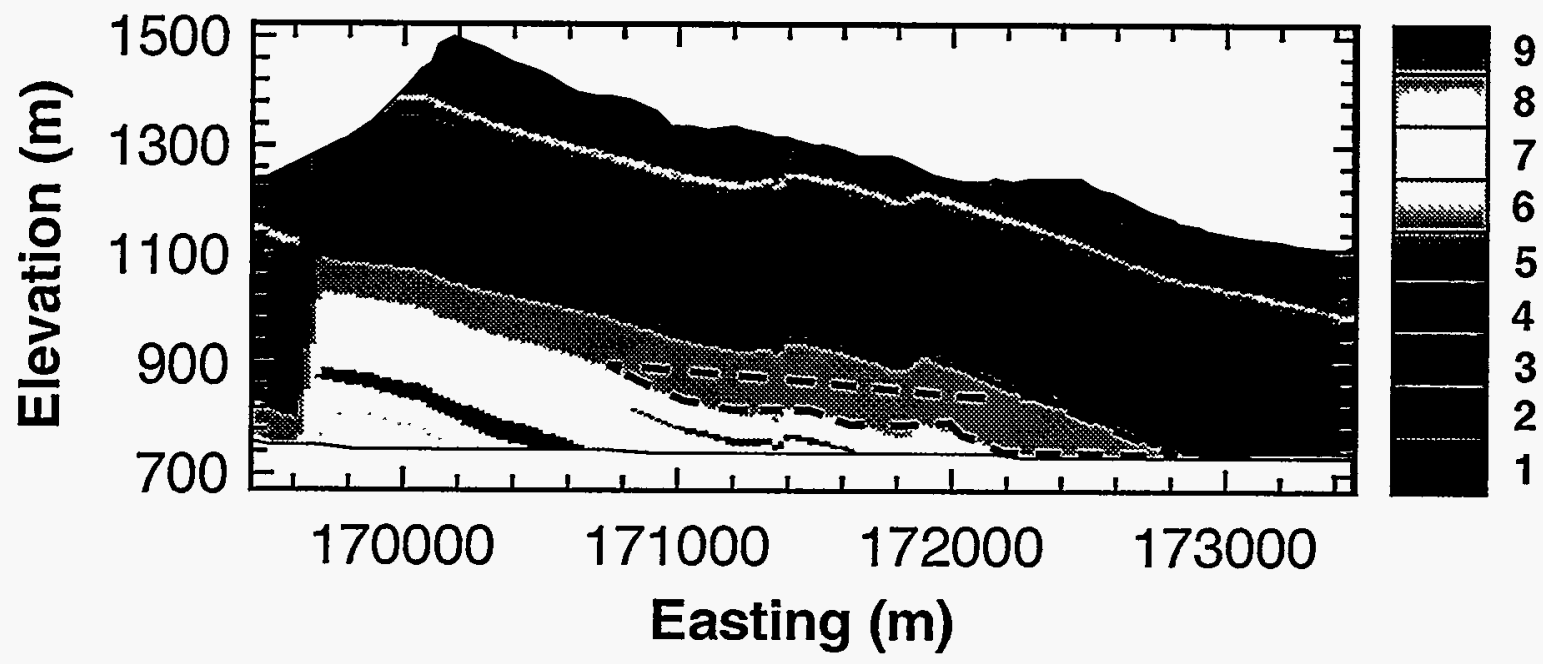

Figure 3-3 Geologic framework model for cross-sections AA (a) and BB (b). The colorscale for the hydrogeological units refers to the hydrogeological unit numbers used in Table 3-1. The area inside the dashed line indicates the region of zeolitization. 
(a)

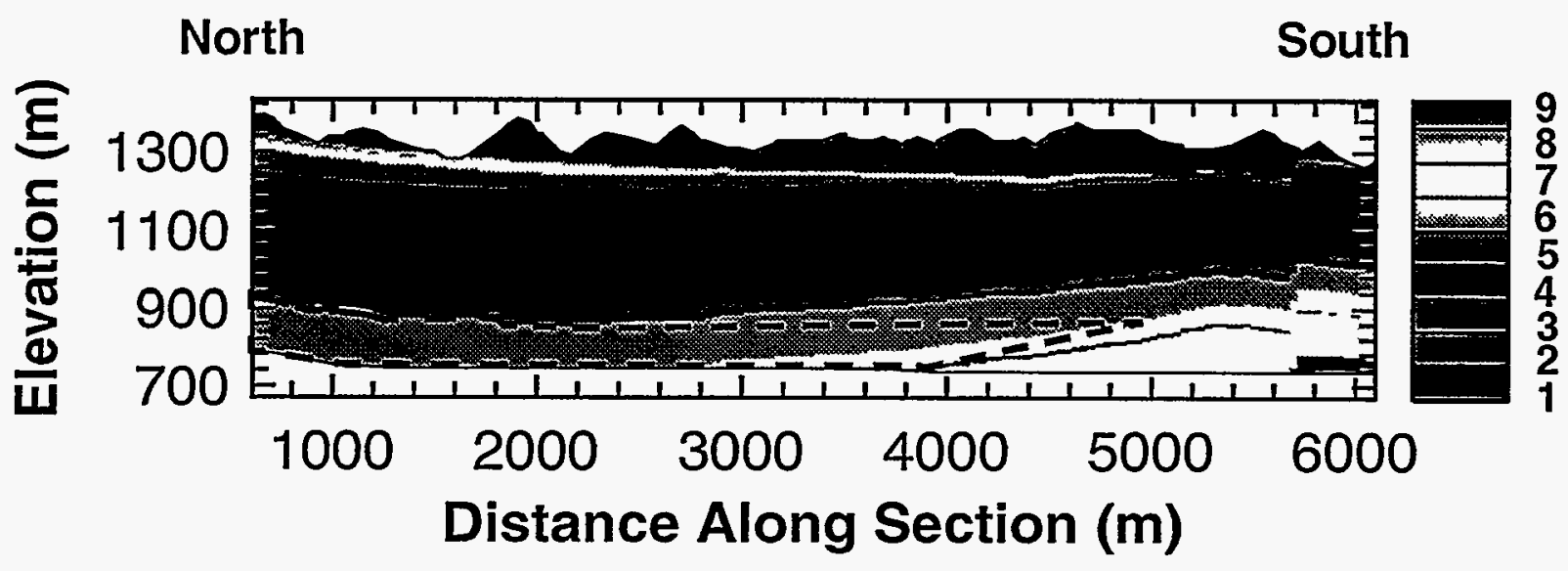

(b) Northwest

Southeast

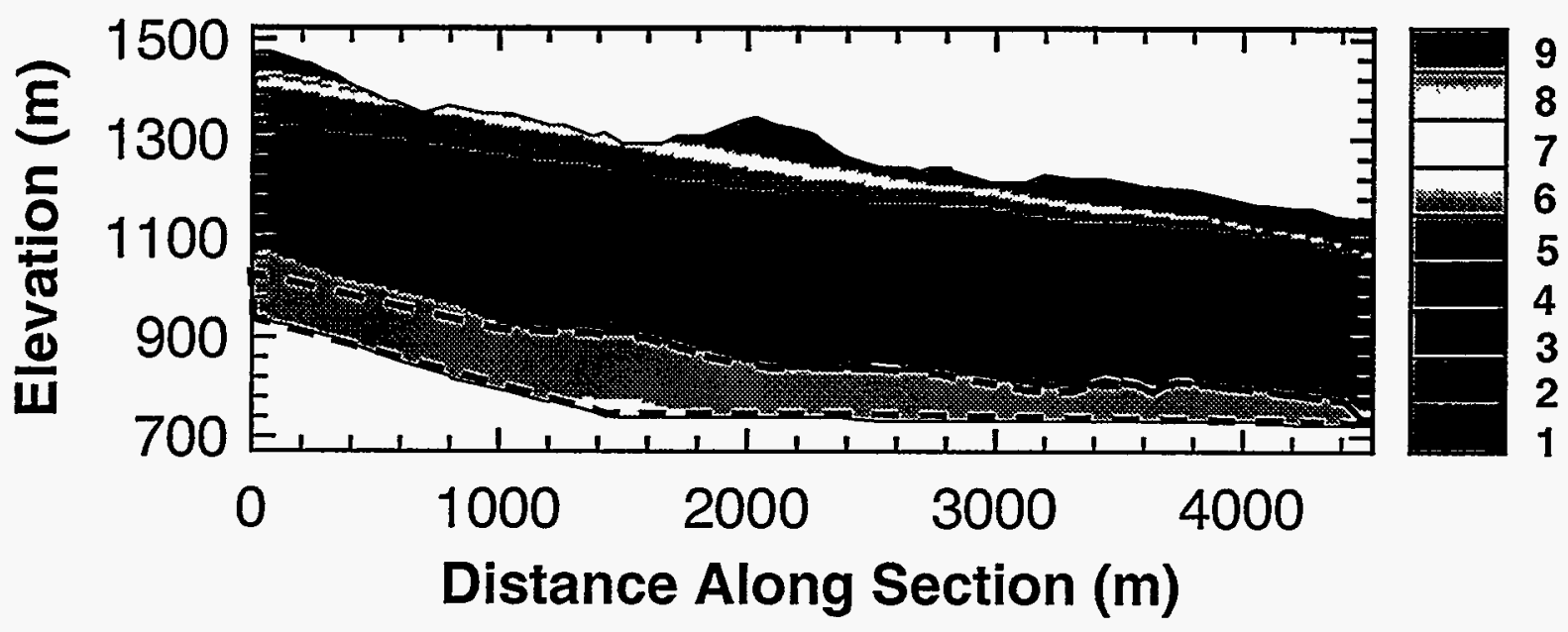

Figure 3-4 Geologic framework model for cross-sections CC (a) and DD (b). The colorscale for the hydrogeological units refers the hydrogeological unit numbers used in Table 3-1. The area inside the dashed line indicates the region of zeolitization. 
To maintain consistency between the mean values for the hydrogeological units used in the framework model and the data conditioning the geostatistical simulations only borings with qualified data were used for the geostatistical simulations. Limiting the conditioning data to qualified data forces the geostatistical simulation to reference the framework geologic model frequently. The geologic framework model is conditioned stratigraphically on all borings.

The zeolitic regions of the non-welded units must be delineated. Alterations due to zeolitization cause a large reduction in the hydraulic conductivity relative to the small reduction in porosity. This difference in hydrologic properties means the unit must be treated differently during the geostatistical simulations (see Sections 3.2.1.2 and 3.3.2.2). Preliminary work has been conducted to delineate this zeolitic region based on published and non-published lithologic logs, geophysical logs, air-filled and pore-filled porosities based on processed geophysical logs (Nelson, 1994) and quantitative mineralogical analyses (Bish and Chipera, 1989 and Chipera et al., 1995). ${ }^{9}$ Based on this work these zeolitic regions are delineated as shown in Figures 3-3 and 3-4.

\subsubsection{Saturated Zone}

The three-dimensional geologic framework model employed in this study for flow simulation in the saturated zone is constructed specifically for the grid used in the flow model. This geologic interpretation is an enhanced version of the geologic model used in TSPA-93 (Wilson et al., 1994). The geologic map at the water table presented in Fridrich et al. (1994) is used as a starting point for the geologic interpretation. Unit contacts are projected to lower layers at approximately $5-10^{\circ}$ dip to the east and are checked for consistency at intersections with wells. The major faults are assumed to be vertical and geologic contacts are truncated at the faults. The geologic model is shown in Figure 3-5 for three of the five layers of the SZ flow model. See Section 5.1.1 for a discussion of the hydrostratigraphic units as defined in the model. Note that the areas to the north of the Yucca Wash structure (shown as dark green in Figure 3-5) and to the west of the Windy Wash Fault (shown in purple in Figure 3-5) are not resolved in the geologic model. Homogeneous material properties are used in each of these areas due to the lack of subsurface information.

\subsection{Hydrological Parameter Data Synthesis and Analysis}

Input parameters required for the flow modeling include porosity, saturated hydraulic conductivity (Ksat) and characteristic curve parameters for the matrix domain and porosity, permeability, aperture, frequency and characteristic curve parameters for the fracture domain. As discussed in Section 3.3.2 matrix porosity and fracture frequency distributions are modeled directly through geostatistical simulation. Matrix Ksat is also calculated at the geostatistical scale using a coregionalization model with porosity (see Section 3.3.2.2). The other parameters are determined through different regression or direct relationships, described below.

9. Buesch, D., personal communication, 1995. 
This page intentionally left blank. 

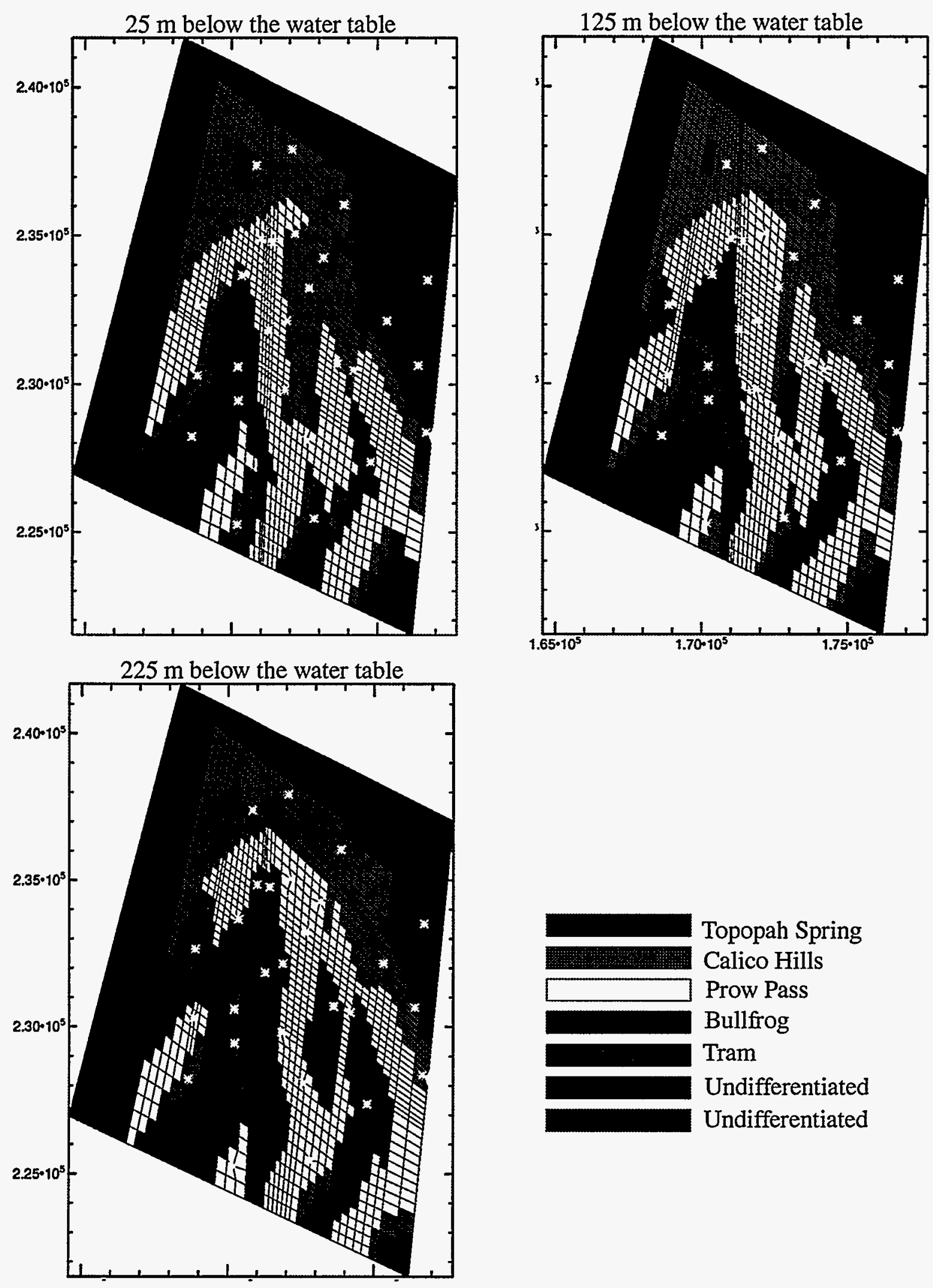

Figure 3-5 Geologic model of the saturated zone shown for three layers, as incorporated into the flow model of the saturated zone. Asterisks indicate wells. 
Input data required for the geostatistical simulations include conditioning matrix porosity and fracture frequency data, where available, as well as the mean and standard deviation of matrix porosity and fracture frequency for the different hydrogeological units described in Section 3.1. The sources of these conditioning data as well as the development of the regression relationships used in the data development are described below.

\subsubsection{Matrix Parameters}

\subsubsection{Porosity}

The porosity measurements used as conditioning data are those that have been determined by methods approved by the quality assurance program (Table 3-2). Measurements made from core from the most shallow neutron boreholes are not used as conditioning data because the only porosity data available from these holes are in the $\mathrm{TCw}$. The neutron holes from which data were used were cored into the upper TSw. Note that there is a gap in data in the southwestern portion of the potential repository site (Figure 3-1). Cross-section C-C has two deep boreholes (SD-9 and SD-12) in its vicinity and therefore will be the most affected by the conditioning data. Crosssection $A-A$ is the least constrained with few boreholes in the vicinity. Data from SD-12 and UZ16 should influence cross-section B-B to some extent, though the borings are not extremely close to the cross-section.

Table 3-2: Matrix porosity conditioning data sources (Refer to Figure 3-1 for borehole locations).

\begin{tabular}{|c|c|c|c|}
\hline SD-9 & UZ-16 & N-32 & N-54 \\
DTN: GS950408312231.004 & DTN: GS940508312231.006 & DTN: GS940108312231.002 & DTN: GS920508312231.012 \\
\hline SD-12 & N-27 & N-37 & NRG-6 \\
DTN: GS950308312231.002 & DTN: GS940108312231.002 & DTN: GS940108312231.002 & DTN: GS950608312231.007 \\
\hline UZ-14 & N-31 & N-53 & \\
DTN: GS950408312231.005 & DTN: GS940108312231.002 & DTN: GS930108312231.006 & \\
\hline
\end{tabular}

\subsubsection{Saturated Hydraulic Conductivity}

In order to perform the coregionalization (see Section 3.3.2.2) to determine the matrix Ksat from porosity, a regression relationship between matrix porosity and matrix hydraulic conductivity is necessary. Data used for determining the relationship between matrix porosity and matrix Ksat are those collected from outcrop transects (Flint et al., 1996) (DTN: GS940808312231.008) and also data from SD-9 (DTN: GS950608312231.006), UZ-16 (DTN: GS960808312231.001) and N-27 (DTN: GS960808312231.001). Only those measurements made from the borehole samples have been qualified. Two separate relationships were determined, one for non- zeolitic units (TCw, PTn, TSw, CHn, PPn, and PPw) and a second for zeolitic units (CHnz and PPnz) (Figure 3-6). The regressions yield the linear relationships presented in Table 3- 
3. With the available data for the zeolitic units, a strong correlation between porosity and Ksat does not exist. However, the error of the regression relationship is modeled when Ksat is calculated using the coregionalization method (see Section 3.3.2.2).

Table 3-3: Regression relationships between porosity and Ksat for zeolitic and non-zeolitic units (see Figure 3-6).

\begin{tabular}{|l|c|c|}
\hline & Regression Equation & $\mathrm{R}^{2}$ \\
\hline \hline Non-Zeolitic Units & $\log _{10}($ Ksat $)=-11.0+11.8(\phi)$ & 0.75 \\
\hline Zeolitic Units & $\log _{10}($ Ksat $)=-11.7+5.8(\phi)$ & 0.15 \\
\hline
\end{tabular}

\subsubsection{Characteristic Curve Parameters}

The van Genuchten method was chosen as the method of fitting both the moisture retention and relative permeability characteristic curves (van Genuchten, 1980). This method was chosen due to availability of data and lack of evidence for a better method.

Recent work has shown considerable variation in the van Genuchten parameters used in different modeling exercises (Wilson, 1996). This comparison has shown that the van Genuchten $\alpha$ parameter can have a significant effect on whether lateral diversion will occur at the interface of the PTn and its bounding welded units. For this reason a review of the available van Genuchten parameter data was made (Table 3-4). There are four sources of data used in Wilson et al. (1994) and Arnold et al. (1995): Peters et al. (1984), Flint and Flint (1990), Rutherford et al. (1992), and Voss (1993). The data reported in Voss (1993) were later reported in Flint et al. (1996) along with some additional data. The data reported in Flint et al. (1996) were obtained from outcrop samples. Finally, the most recent data were taken from core samples in UZ-16 and N-27; these data have not yet been published, but are the only available quality assured data available at this time. ${ }^{10}$

With the exception of Flint and Flint (1990), each referenced study reported using a psychrometer to determine the moisture retention curve (Table 3-4). Based on the fact that different methods were used in Flint and Flint (1990) it was decided not to use these data for the GWTT-95 calculations. Examination of $\alpha$ for the remaining references shows that in the PTn and somewhat in the TSw the mean $\log (\alpha)$ is lower in the Peters et al. (1984) and Rutherford et al. (1992) data sets than those of Flint et al. (1996) and Flint ${ }^{11}$ (Figure 3-7). These differences can be explained by the different sample preparation methods used in each study. Peters et al. (1984) let the samples equilibrate for only one hour after partial drying in a microwave oven. This drying process only dries the outside of the sample, time is then needed for the moisture content to equilibrate throughout the whole sample. After equilibration a relative humidity measurement is made indicating the water potential. If the sample is not given enough time to equilibrate, as is the case with Peters et al. (1984), then the relative humidity reading will be too low, thus the water

10. Flint, L.E., personal communication, 1995.

11. Ibid. 


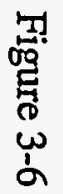
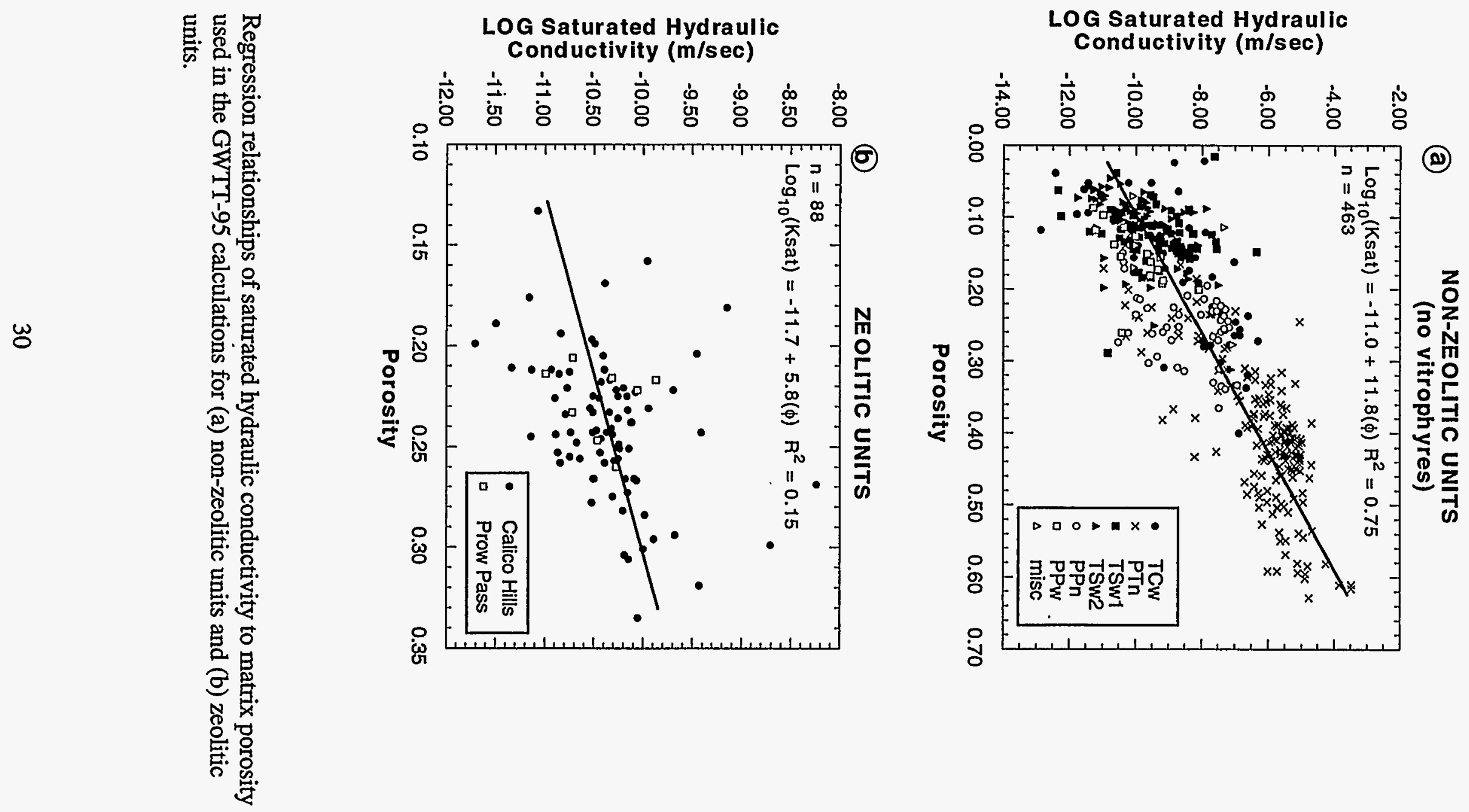

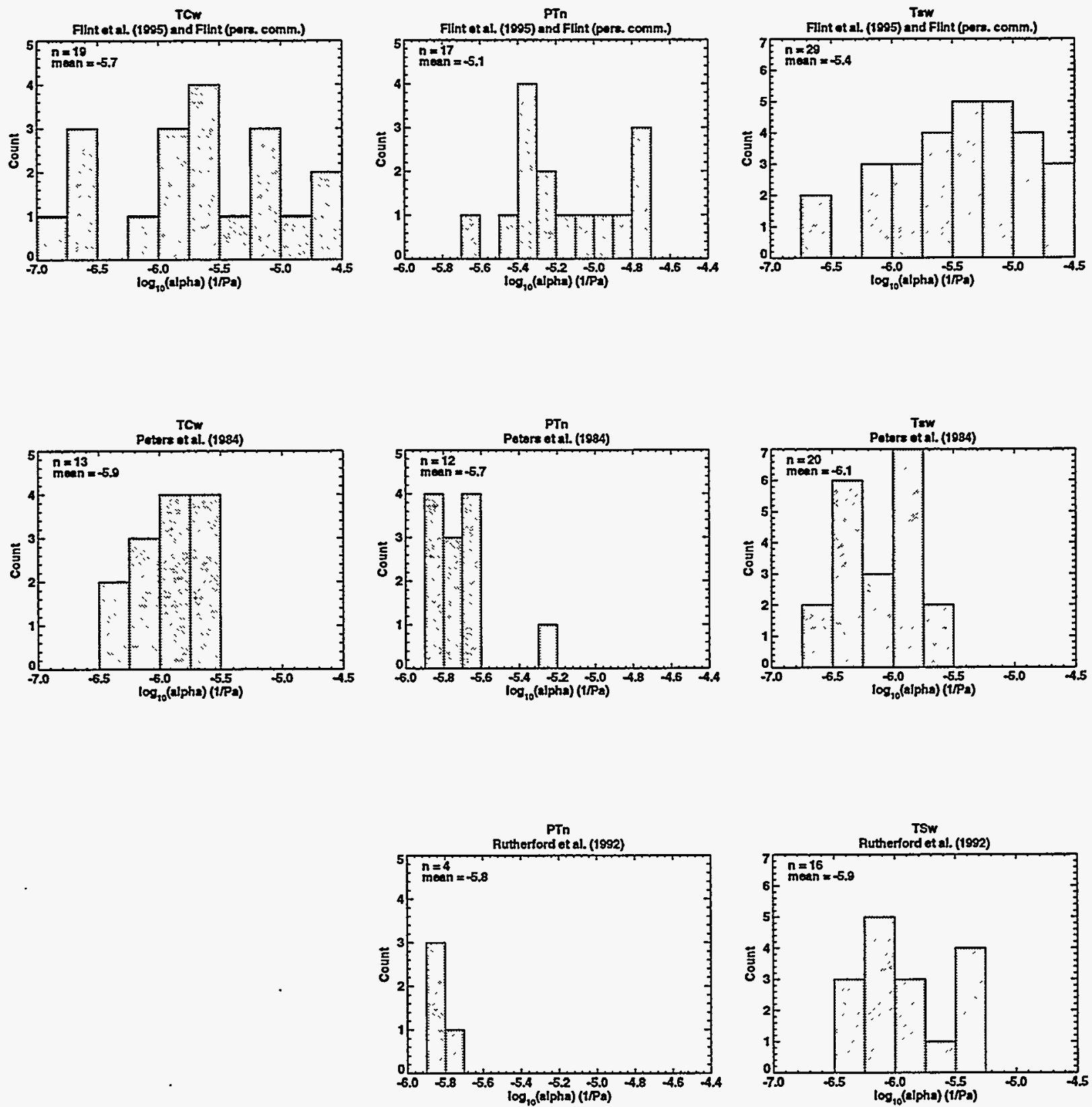

Figure 3-7 Distribution of van Genuchten $\alpha$ for the upper three hydrogeological units (TCw, PTn and TSw) from three different sources (data for $\mathrm{TCw}$ were not available from Rutherford et al., 1992). Note that the approximate mean $\log (\alpha$ 's) are higher in the data from Flint et al. (1996) and Flint (see footnote 10 , page 29) than the other two references. 
Table 3-4: Summary of available van Genuchten parameter data.

\begin{tabular}{|l|c|l|l|c|}
\hline & $\begin{array}{c}\text { Number of } \\
\text { Samples }\end{array}$ & \multicolumn{1}{|c|}{ Cores } & Methods & $\begin{array}{l}\text { Data } \\
\text { QAed? }\end{array}$ \\
\hline \hline $\begin{array}{l}\text { Peters et al. } \\
(1984)\end{array}$ & 107 & G-4, GU-3 & $\begin{array}{l}\text { thermocouple } \\
\text { psychrometer }\end{array}$ & No \\
\hline $\begin{array}{l}\text { Flint and Flint } \\
(1990)\end{array}$ & 30 & $\begin{array}{l}\text { G-1, UZ-5, UZ-4, } \\
\text { UE-25a \#6, } \\
\text { UE-25a \#1, } \\
\text { UE-25c \#1, GU-3 }\end{array}$ & $\begin{array}{l}\text { 1) porous plate using } \\
\text { a pressure extractor } \\
\text { 2) Mercury Intrusion } \\
\text { 3) centrifugation }\end{array}$ & No \\
\hline $\begin{array}{l}\text { Rutherford et al. } \\
(1992)\end{array}$ & 40 & G1, GU3, G4 & Psychrometer & No \\
\hline $\begin{array}{l}\text { Flint et al. } \\
(1996)\end{array}$ & 41 & Transect Data & $\begin{array}{l}\text { chilled mirror } \\
\text { psychrometer }\end{array}$ & No \\
\hline Flint ${ }^{\mathrm{a}}$ & 55 & UZ-16, N-27 & $\begin{array}{l}\text { chilled mirror } \\
\text { psychrometer }\end{array}$ & Yes \\
\hline
\end{tabular}

a. Flint, L. E., personal communication, 1995

pressure will be too negative which will mean the air entry pressure will be too high and thus $\alpha$ too low. This is exactly what is observed in the data (Figure 3-7). It is expected that this difference would become more exaggerated in the welded units if reaching equilibrium is only affected by the permeability of the sample (the lower the permeability, the more time needed to reach equilibrium). The trend of lower $\alpha$ 's can be observed in the TSw, but is most obvious in the PTn. Measurements of $\alpha$ in the non-welded units are complicated by the intricate pore structure in these units containing three distinct ranges of pores sizes as described by Peters et al. (1987). Thermocouple-psychrometer methods measure only smaller pores in the range of 0.03 to $0.3 \mu \mathrm{m}$ (Peters et al., 1987). Thus, examining these data suggest that when the sample is set to equilibrate the moisture might move preferentially into the larger pore spaces, thus the smaller pores that the thermocouple-psychrometer measures become inproportionately dry. Beta values proved to be consistent between the different sources of data (Figure 3-8).

The sample preparation methods used by Flint et al. (1996) and Flint ${ }^{12}$ avoids the concern of when the moisture content of the sample will be in equilibrium and, therefore, appears to produce more accurate relative humidity readings and therefore more accurate $\alpha$ values. In this case samples are allowed to air dry on a scale until the desired moisture content is reached. Then the relative humidity measurement in the chilled-mirror psychrometer is made over a period of time so that an equilibrium dew point is reached.

12. see footnote 10 , page 29 . 

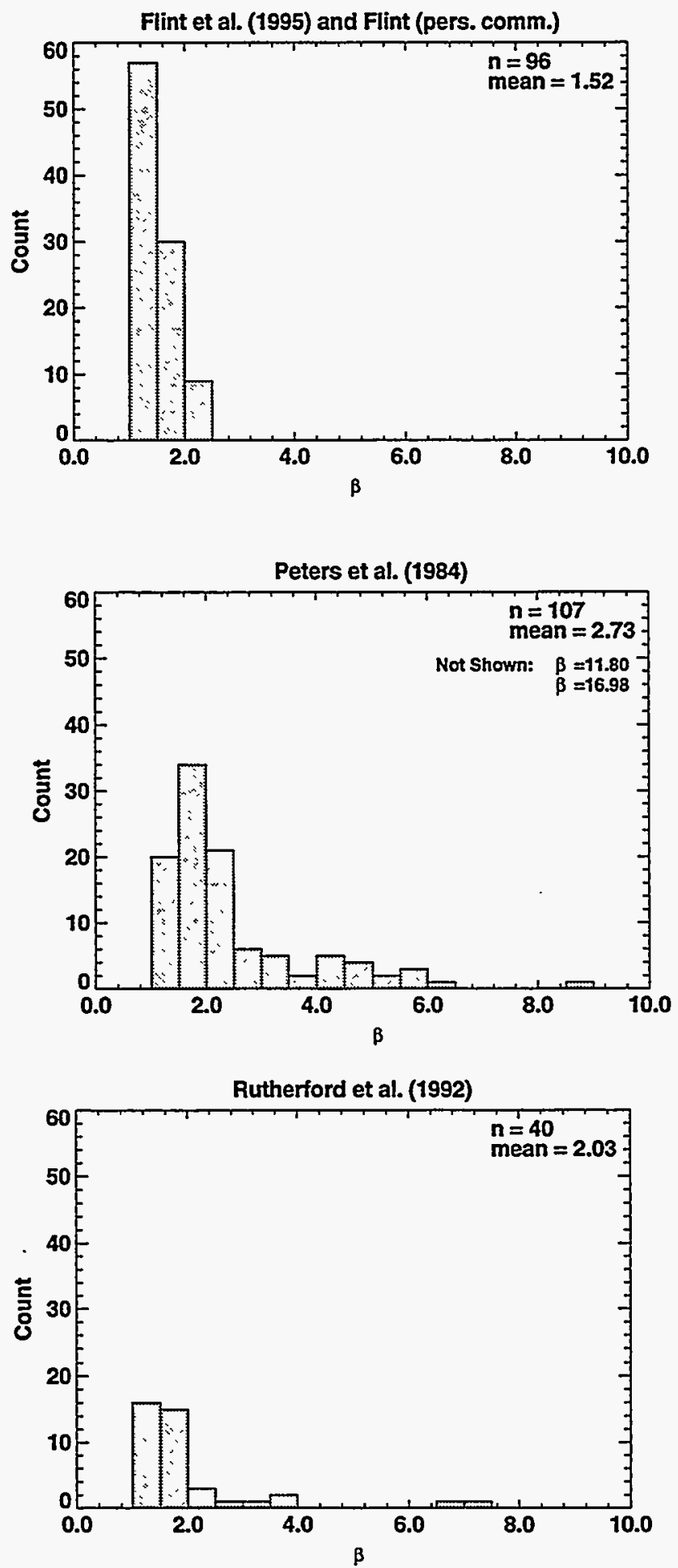

Figure 3-8 Distribution of van Genuchten $\beta$ for the available data from three different sources. 
The determination of van Genuchten parameters was also improved by including in-situ saturation and pressure head data measured directly from borehole core samples for 25 hydrogeological layers defined in Flint ${ }^{13}$ to provide "average" van Genuchten parameters for each layer. The average parameters were determined from van Genuchten curves for each hydrogeological unit layer fit on all of the available lab and in-situ data for that layer. These average curves were created using the data from UZ-16. Saturations calculated from the average curves using in-situ pressure head data from SD-9, SD-12 and SD-14 closely match the measured in-situ saturations (Flint ${ }^{14}$ ). The van Genuchten $\alpha$ determined in the laboratory on individual core samples (the lab data) are generally larger than the average $\alpha$ for each hydrogeological layer, especially in the PTn (Figure 3-9). The larger values in PTn can be explained by the methods used to measure water potential. Air entry pressures lower than 1 bar cannot be measured accurately with the present equipment which only has a resolution of $+/-1.26$ bars. Thus the "wet" end of the characteristic curves of these samples are inaccurate. In addition, three of the samples from the PTn could not be sub-sampled to provide coherent cores for the saturation-pressure data (Flint ${ }^{15}$ ). The relatively large differences between the lab data $\alpha$ and the average $\alpha$ calculated using the insitu saturations indicate that the larger pore sizes not measured with the psychrometer are important to the flow system and resulting saturations.

Matrix $\alpha$ for the flow model is determined using a regression relationship with matrix Ksat (Figure 3-10). Note that the data used for this regression are preliminary, but are the best available data at the time of the analyses. The final versions of these parameters will be published in Flint ${ }^{16}$. The average $\alpha$ 's described above are used because it is believed that these data better represent the field data and thus produce more accurate models. Two data points are not used in this regression; both points represent units at the base of the TCw. Both samples have a high clay content. While this clay content is a real phenomenon, these relatively thin layers $(6 \mathrm{~m}$ and $5 \mathrm{~m}$ ) are not represented in our model. Including these points in the regression would thus compromise the predictions of $\alpha$ for the PTn.

Using the same points that were used in the $\alpha$-Ksat regression, a mean $\beta$ of 1.60 (standard deviation $=0.394$ ) is calculated (Figure 3-12). This slightly higher mean than that calculated using the lab data (which had a mean of 1.52 and a standard deviation of 0.25 ) is accounted for by two higher values, one from the Calico Hills vitric unit and the second from a bedded tuff unit directly above the Calico Hills ( 3 and 2.5 , respectively). As this data set is preliminary, it is highly likely that as more data become available these values will change. Until then there is not a justification for taking them out of the data set and they do not influence the mean significantly.

In conclusion, the following regression between saturated hydraulic conductivity $(\mathrm{m} / \mathrm{s})$ and $\alpha(1 / \mathrm{Pa})$ is used for the GWTT-95 calculations:

$$
\log _{10}(\alpha)=-1.62+0.41 \log _{10}(\text { Ksat }) \quad R^{2}=0.81
$$

13. Flint, L. E., (in prep.), Matrix properties of hydrogeologic units at Yucca Mountain, Nevada, U.S. Geological Survey Water Resources Investigation Report, Denver, CO.

14. see footnote 10 , page 29 .

15. see footnote 10 , page 29 .

16. see footnote 13 above 

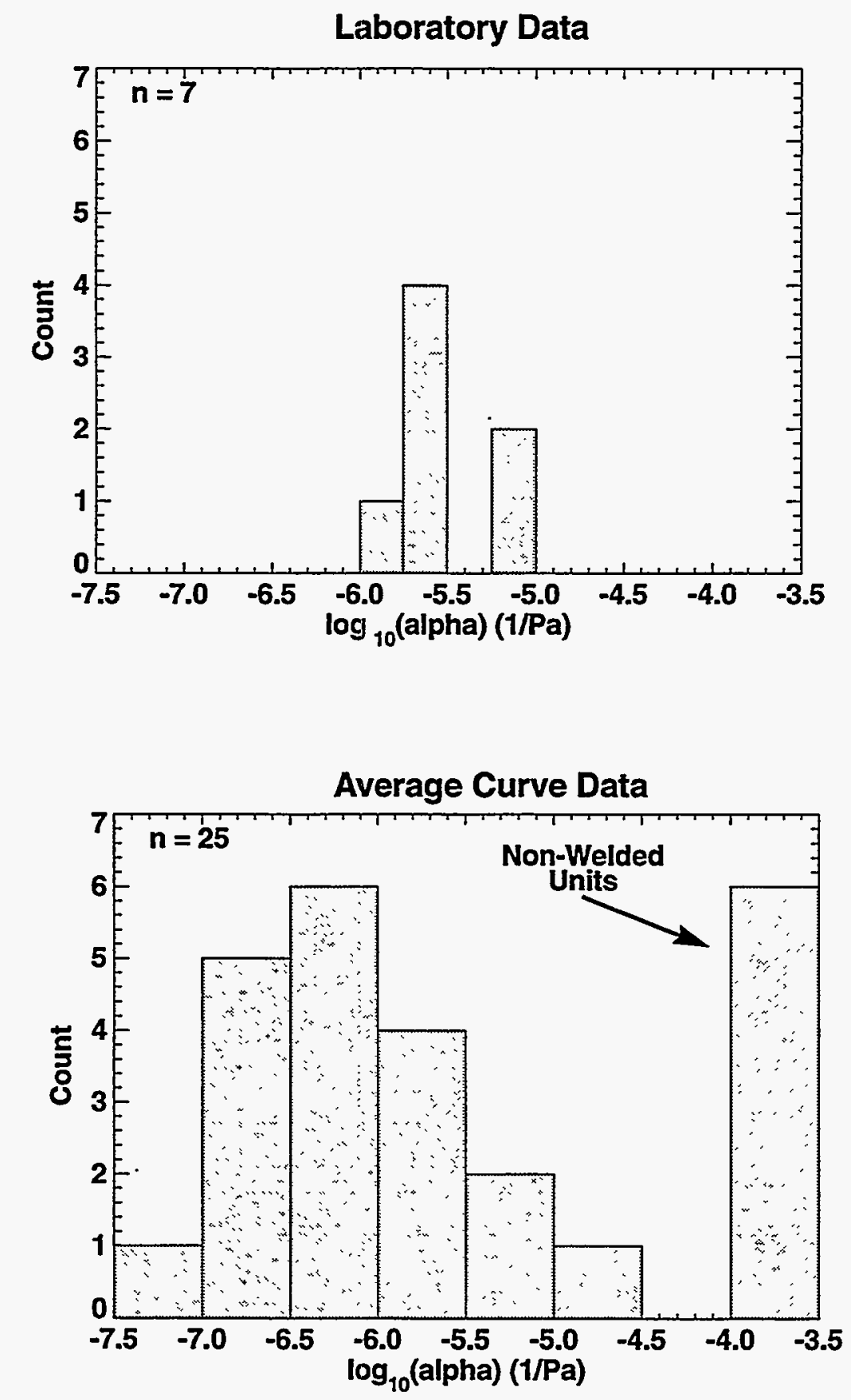

Figure 3-9 Comparison of the relationship of raw $\alpha$ data determined on laboratory samples to the average $\alpha$ 's calculated using in-situ and laboratory data. Values of lab data $\alpha$ 's are averages for the GWTT-95 hydrogeological units. Note the $\alpha$ 's in the non-welded units are significantly higher after calibration. 


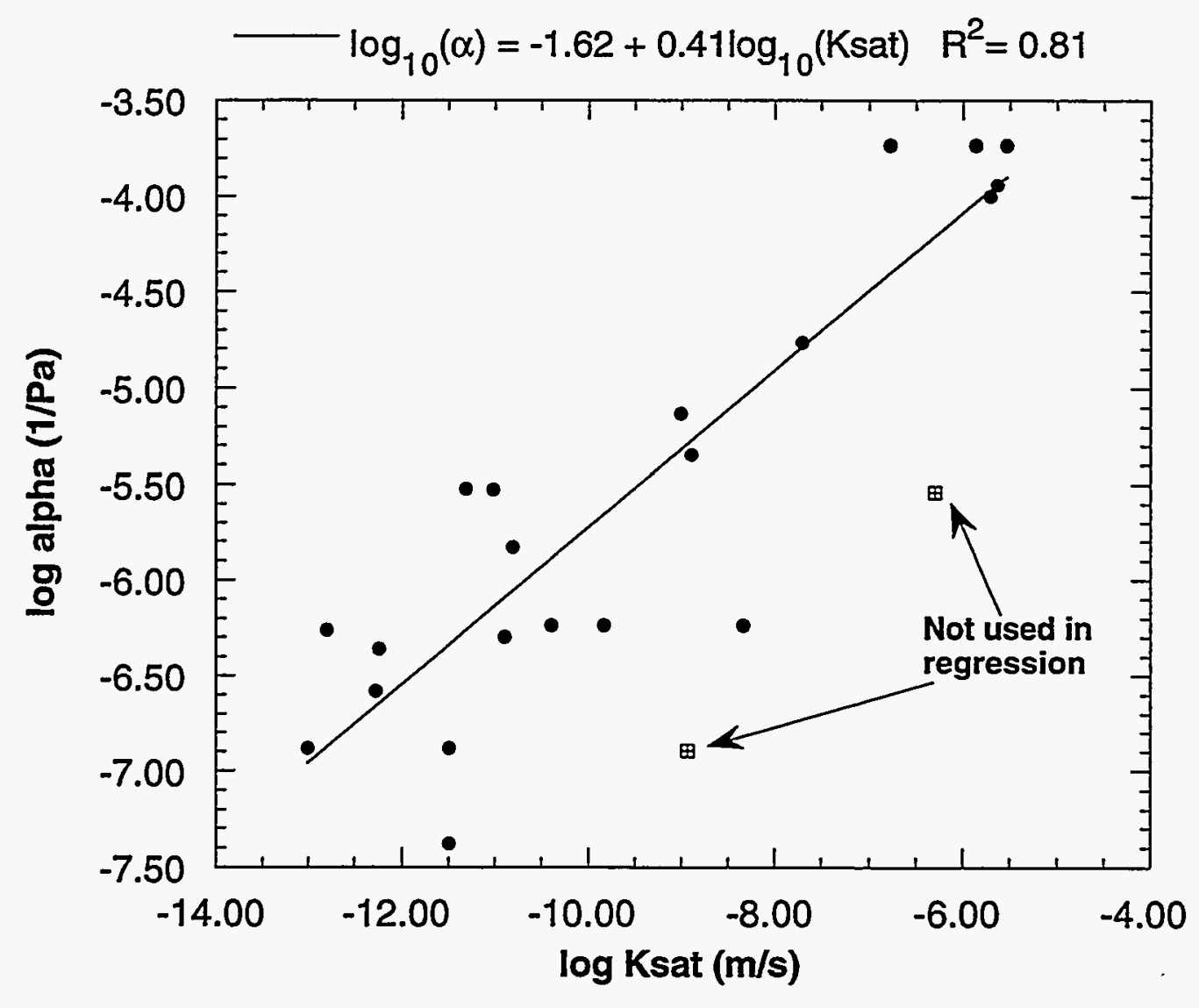

Figure 3-10 Regression relationship of matrix $\alpha$ to saturated hydraulic conductivity used for the GWTT-95 calculations.

Because the range in $\beta$ is only from 1.21 to 1.73 (excluding the anomalous points), a constant value of 1.60, the mean, is used for the GWTT-95 calculations (Figure 3-12). A constant residual saturation of 0.0453 was used in the GWTT-95 simulations taken from Wilson et al. (1994) as the expected residual saturation from the TSw.

As an exercise to visualize the average characteristic curves for the upper units, the mean porosity for the units in our GWTT-95 model are used to calculate Ksat using the regression between Ksat and porosity (Figure 3-6). An average $\alpha$ is then calculated using the regression equation above. Residual saturation is set at 0.0453 as is used for the modeling. It appears that there could be lateral diversion above the PTn if the infiltration is greater than approximately 0.6 $\mathrm{mm} / \mathrm{yr}$ (Figure 3-12). Diversion could occur at the base of the PTn if infiltration is greater than approximately $0.5 \mathrm{~mm} / \mathrm{yr}$ if the TSw caprock is present or greater than approximately $1.7 \mathrm{~mm} / \mathrm{yr}$ if the PTn contacts the TSw non-lithophysal unit (Figure 3-12). These necessary minimal infiltration rates are higher than those used by Prindle and Hopkins (1990), Wilson et al. (1994) and Arnold et al. (1995). However, more recent studies (Hudson and Flint ${ }^{17}$; Hevesi and Flint ${ }^{18}$ )

17. see footnote 2 , page 9 .

18. see footnote 3 , page 9 . 


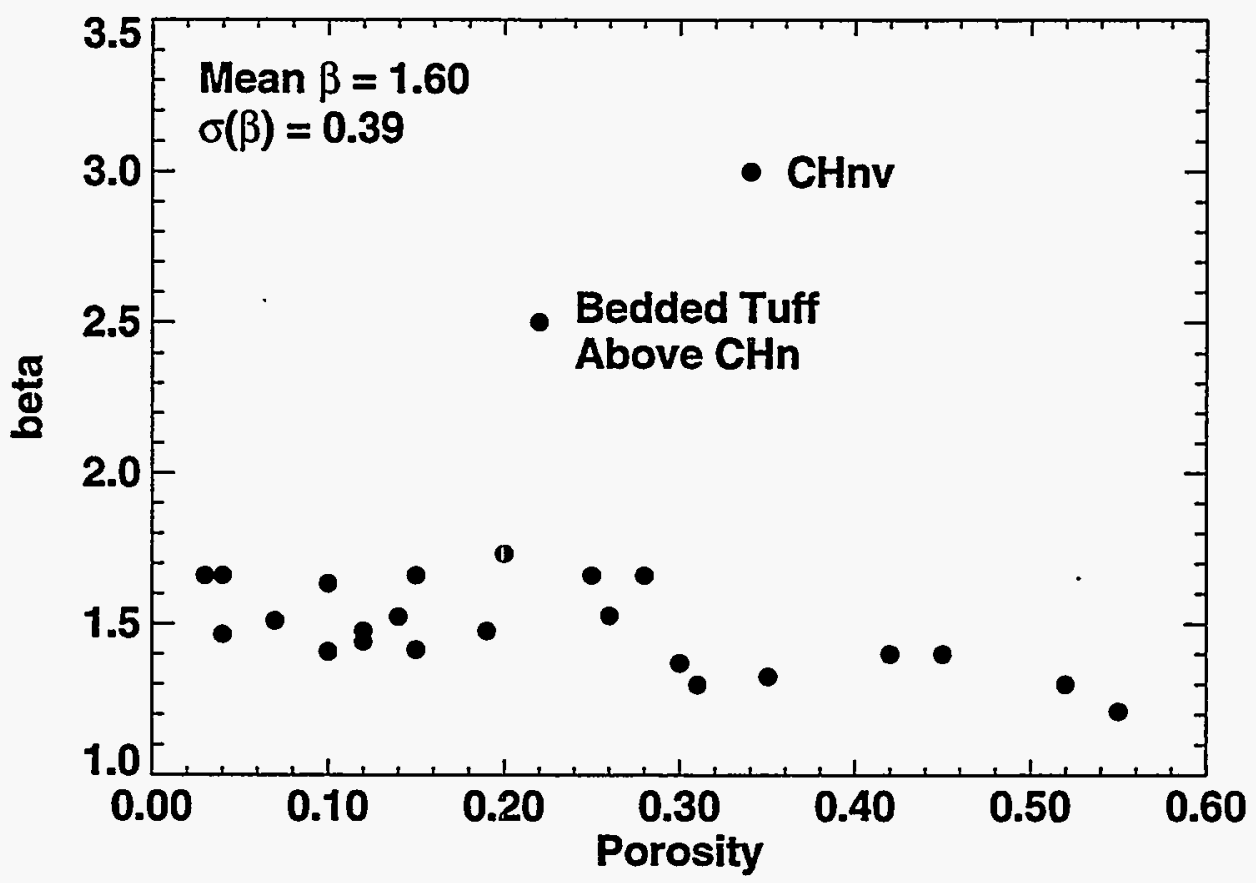

Figure 3-11 Relationship of matrix $\beta$ to porosity. A constant $\beta$ value of 1.60 is used for the GWTT-95 calculations.

indicate that infiltration rates could be in this range or even higher. Predicting whether water will be laterally diverted in the GWTT-95 modeling is further complicated by the fact that the system is heterogeneous.

\subsubsection{Fracture Properties}

\subsubsection{Fracture Frequency}

Fracture frequency data, measured as the number of fractures in a ten foot interval of core, are taken from the boreholes listed in Table 3-5 and are used both as conditioning data and to determine the mean and standard deviation of fracture frequency assigned to each hydrogeological unit. These data are qualified. In addition to these core data, fracture frequencies measured on three pavements, all in the TCw upper lithophysal, are used as conditioning data (Barton et al., 1993) (DTN: GS940608314222.002) (Figure 3-1). Fracture frequencies are calculated as the total length of fractures in the pavement divided by the area of the pavement. Note that, except for SD-9 and SD-12, the cores from which the rest of the fracture frequency data are derived are located in Drill Hole wash. It is therefore likely that these data are biased towards higher fracture frequencies due to the Drill Hole Wash structure. 
(a)

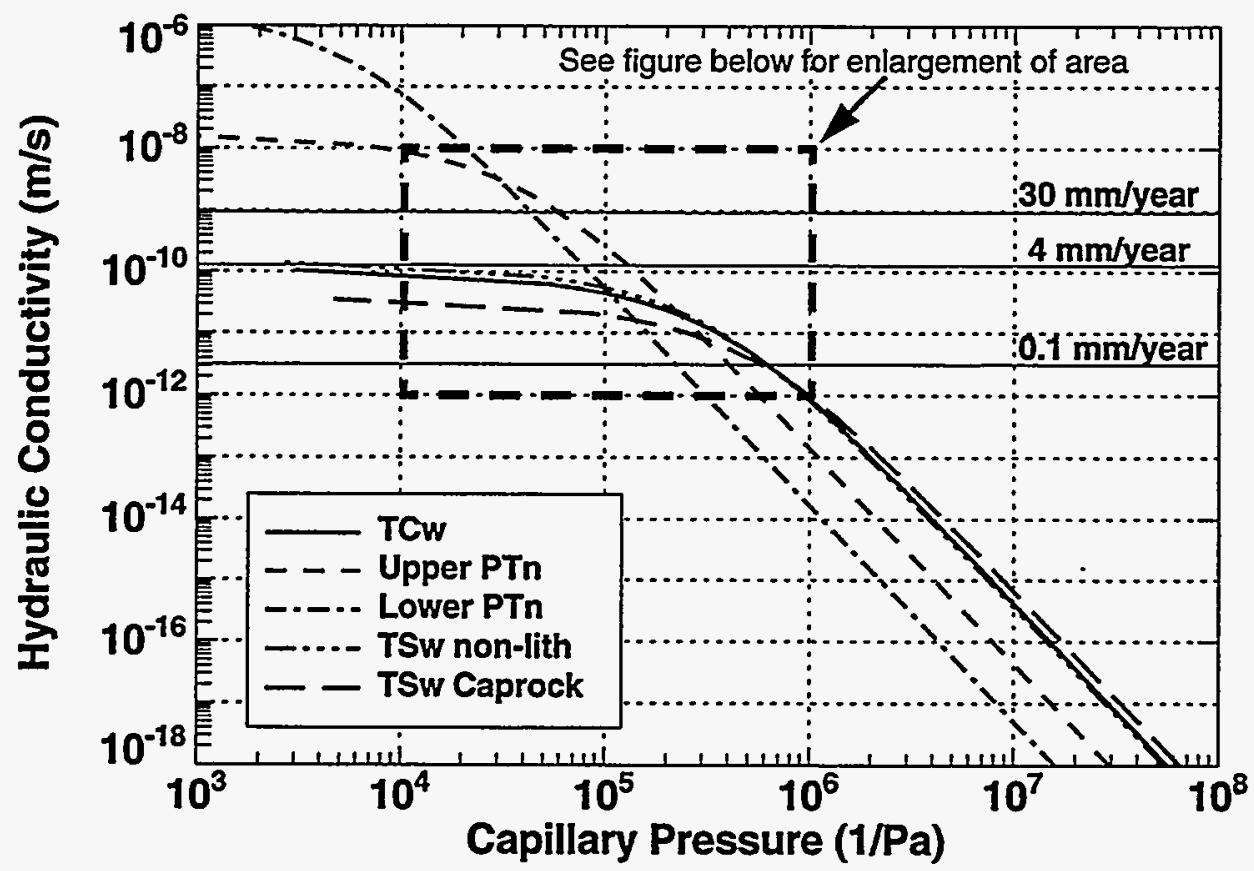

(b)

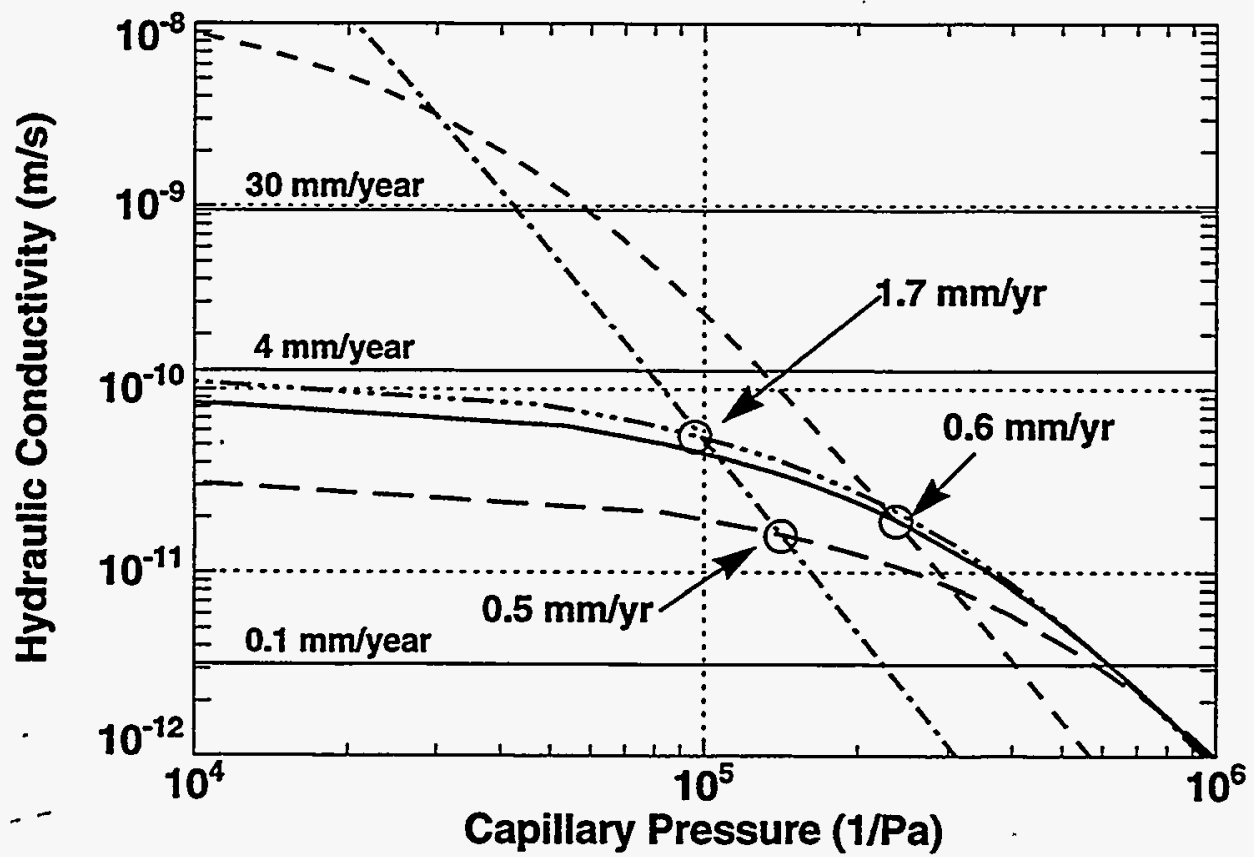

Figure 3-12 Unsaturated characteristic curves for the TCw, PTn, and TSw using the mean matrix van Genuchten parameters, mean porosity and saturated hydraulic conductivity for each hydrostratigraphic unit. The lower graph (b) is an enlargement of the delineated area in the upper graph (a). 
Table 3-5: Fracture frequency data sources.

\begin{tabular}{|c|c|c|}
\hline NRG-4 & NRG-6 & SD-9 \\
DTN: SNF29041993002.048 & DTN: SNF29041993002.048 & DTN: SNF29041993002.069 \\
\hline NRG-5 & NRG-7/7A & SD-12 \\
DTN: SNF29041993002.048 & DTN: SNF29041993002.048 & DTN: SNF29041993002.071 \\
\hline
\end{tabular}

Raw fracture frequency data can not be used because of two forms of bias in the data. First, a vertical borehole is more likely to intersect a horizontal or gently dipping fracture than a vertical or steeply dipping fracture. Therefore, if a vertical borehole intersects a steeply dipping fracture it should be given more weight in the fracture frequency calculation than a gently dipping fracture. The following equation was used to do this weighting:

Corrected Fracture \# $=\frac{\# \text { of Fractures with dips between } 0 \text { and } 10^{\circ}}{\cos 5}+\frac{\# \text { of Fractures with dips between } 10 \text { and } 20^{\circ}}{\cos 15}+$
$\frac{\# \text { of Fractures with dips between } 20 \text { and } 30^{\circ}}{\cos 25}+\frac{\# \text { of Fractures with dips between } 30 \text { and } 40^{\circ}}{\cos 35}+$
$\frac{\# \text { of Fractures with dips between } 50 \text { and } 60^{\circ}}{\cos 55}+\frac{\# \text { of Fractures with dips between } 60 \text { and } 70^{\circ}}{\cos 65}+$
$\frac{\# \text { of Fractures with dips between } 70 \text { and } 80^{\circ}}{\cos 75}+\frac{\# \text { of Fractures with dips between } 80 \text { and } 90^{\circ}}{\cos 85}$

A similar correction to raw fracture frequency data was made by Spengler et al. (1984)

An interval of $10^{\circ}$ was chosen because at the time it was thought that average fracture frequency data from Spengler et al. (1981 and 1984) and Scott and Castellanos (1984), reported in $10^{\circ}$ intervals for each hydrogeological unit could be included. In the end, these data were not used due to insufficient information on the fracture dips. For the data that are used the dip is reported for individual fractures.

The second form of bias in the raw data is that differences in the amount of core recovered from each run gives some intervals higher weighting than others. To correct for this bias, the measured fracture frequencies are divided by the percent recovery for that interval. Therefore, if only half of the core length is recovered the fracture frequency is doubled. If less than $10 \%$ of the interval is recovered for a run then the fracture frequency is taken to be the average of the fracture frequencies for intervals above and below the run.

An example of a fracture frequency distribution before and after the above two corrections are made is presented in Figure 3-13. It can be seen that the fracture frequencies are increased significantly by these correction methods. However, the trends of higher and lower frequencies 
remain similar. The large resulting frequencies do not seem unrealistic, but should be compared with data from the Exploratory Studies Facility (ESF) as it is made available. It should be noted that dividing the fracture frequency data in $10^{\circ}$ increments based on dip results in a greater increase in fracture frequency than if a larger increment (i.e. $30^{\circ}$ ) is used. An evaluation of the sensitivity to dip increments to use is therefore useful (see Section 4.5.5).

The core data show that there are some 10 foot intervals with 0 fractures. To quantify these low fracture frequency intervals, the percentage of intervals without fractures is calculated for each hydrostratigraphic unit. This percentage is the likelihood of hitting an interval without a fracture. The mean fracture frequency assigned to each hydrostratigraphic unit along with the probability of hitting a 10 foot interval without a fracture are reported in Table 3-6. Note that fracture data are not available from the Prow Pass welded and non-welded units. The PPw is assigned a fracture frequency as an average between the TSw lithophysal and nonlithophysal. The PPnw is assigned the same fracture frequency as the PTn.

Table 3-6: Mean fracture frequency and the probability of hitting a 10 foot interval without a fracture for each hydrogeological unit.

\begin{tabular}{|l|c|c|}
\hline Hydrostratigraphic Unit & $\begin{array}{c}\text { Mean Fracture } \\
\text { Frequency }(1 / \mathrm{m})\end{array}$ & $\begin{array}{c}\text { Probability of Hitting a 10 foot } \\
\text { Interval without a Fracture }\end{array}$ \\
\hline \hline TCw & 14.16 & 0.0 \\
\hline PTn & 2.92 & 0.22 \\
\hline TSw nonlithophysal & 16.93 & 0.015 \\
\hline TSw lithophysal & 15.98 & 0.086 \\
\hline CHn & 2.22 & 0.46 \\
\hline vitrophyres & 11.68 & 0.071 \\
\hline
\end{tabular}

\subsubsection{Fracture Porosity}

Fracture porosity is calculated using a regression relationship between the median matrix porosity and mean fracture porosity for each hydrogeological unit (Figure 3-14). This relationship is consistent with observations of the relationship between degree of welding and fracture frequency described in Section 2.1.1, in which the more brittle, welded units have higher fracture frequency and fracture porosity. From their relationship, a fracture porosity is calculated based on the geostatistically simulated matrix porosity. Values of fracture porosity used in the regression are calculated for each unit using the fracture frequencies described above and expected fracture aperture values reported in Wilson et al. (1994):

$$
\phi_{\mathrm{f}}=\mathrm{bF}_{\mathrm{f}}
$$



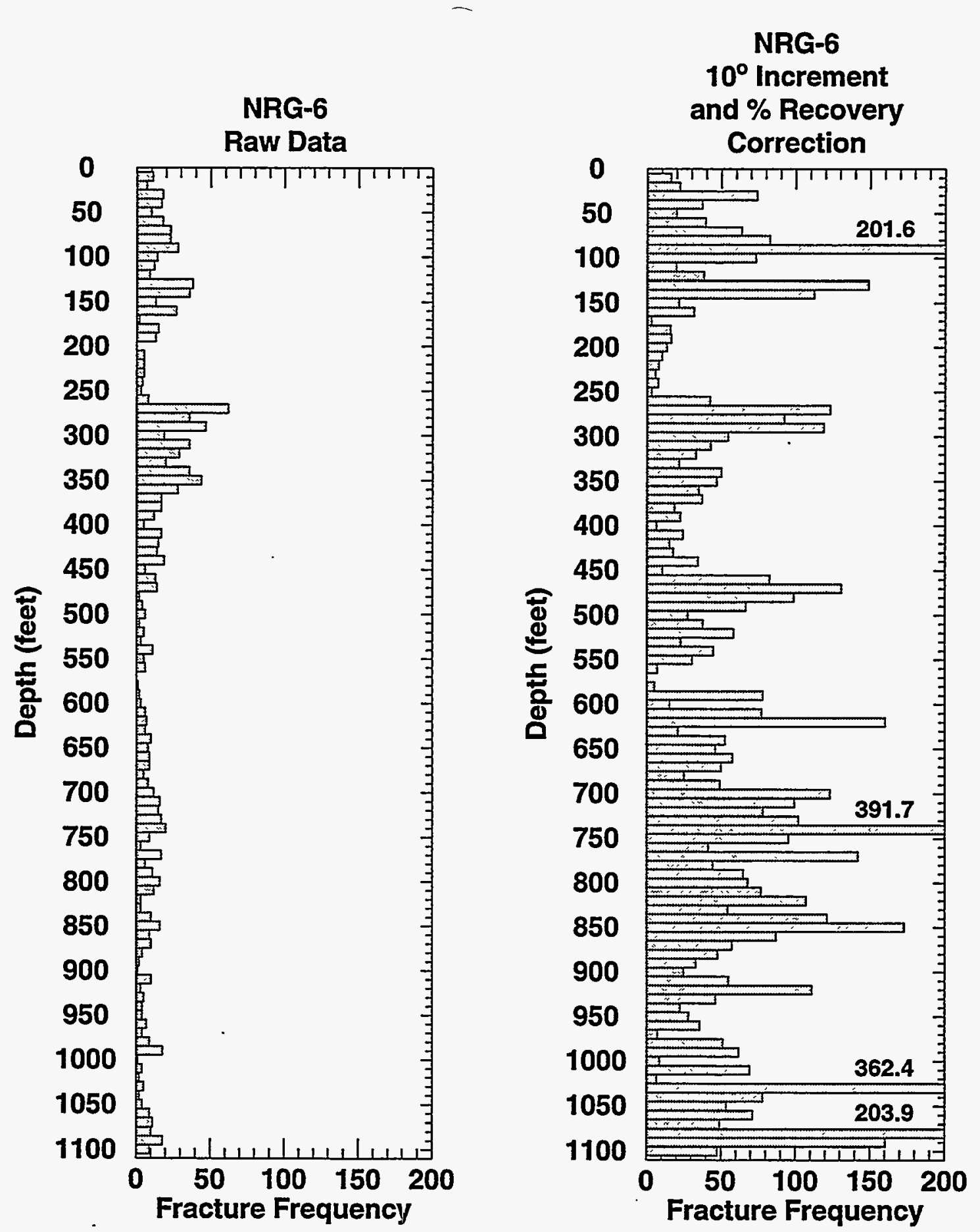

Figure 3-13 Fracture frequency data before and after corrections for bias due to vertical boreholes and varying recovery rates for borehole NRG-6. 


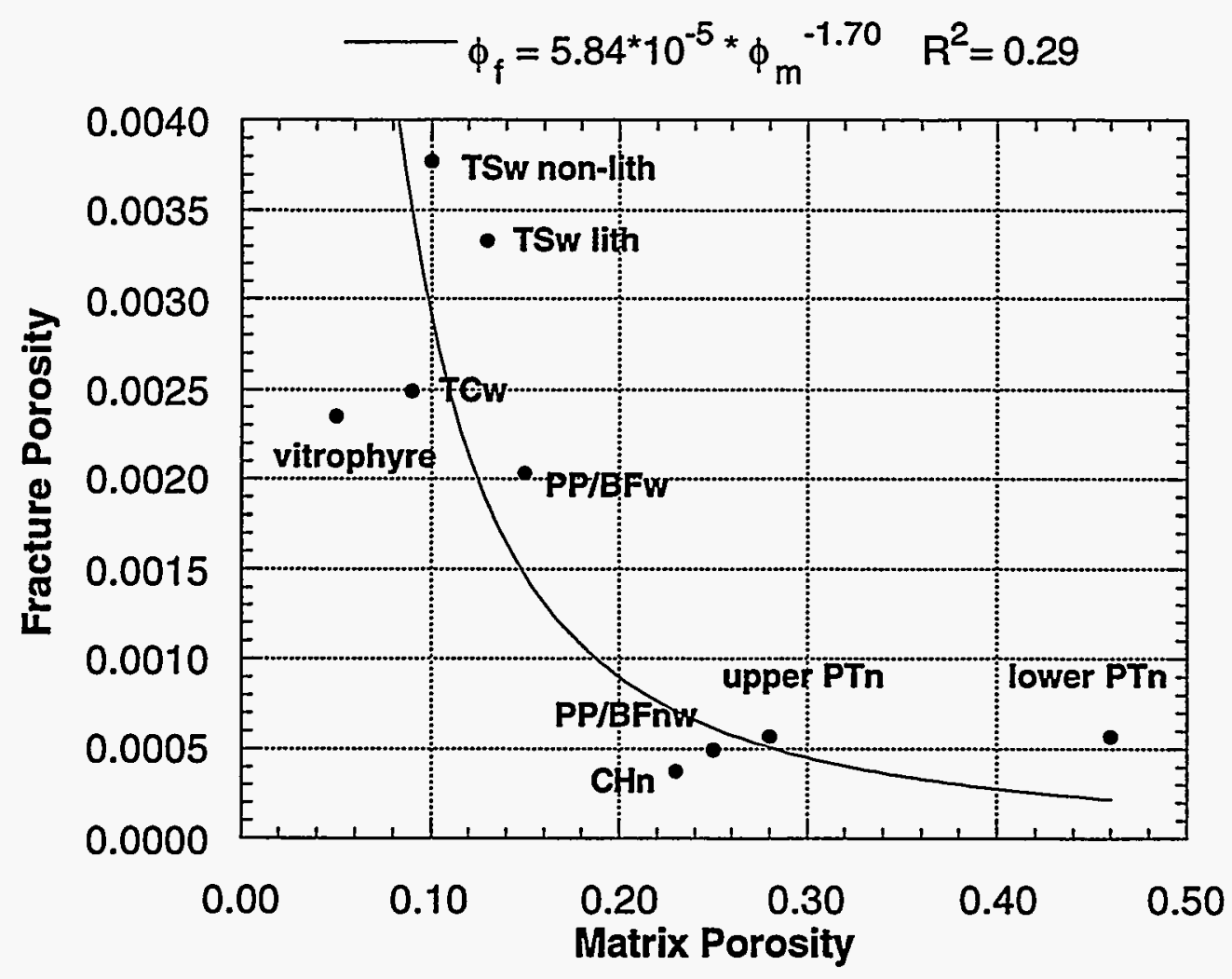

Figure 3-14 Regression relationship between matrix and fracture porosity used for GWTT-95 calculations. This regression relationship is adjusted to preserve the fracture aperture distribution, as described in the text.

where $\phi_{\mathrm{f}}=$ fracture porosity

$\mathrm{b}=$ fracture aperture $(\mathrm{m})$

$F_{\mathrm{f}}=$ Fracture frequency $(1 / \mathrm{m})$

The fracture aperture values used are listed in Table 3-7. These are expected values determined from beta distributions (see Wilson et al., 1994). The range is between 103 and 206 microns. After fracture apertures are determined for a cross-section the aperture distributions are examined to determine if the apertures still have these general means (Section 3.2.2.3). It was discovered, that depending on the distributions of the different hydrogeological units in a crosssection, the mean aperture for the entire cross-section might be well under what is expected based on the Wilson'et al. (1994) data. In order to correct for this, the equation for the regression shown in Figure 3-14 is multiplied by 2 for cross-sections A-A, B-B and C-C and by 1.25 for crosssection D-D. 
Table 3-7: Expected fracture aperture of each hydrogeological unit (Wilson et al., 1994).

\begin{tabular}{|l|c||l|c|}
\hline \multicolumn{1}{|c|}{ Unit } & $\begin{array}{c}\text { Fracture Aperture } \\
\text { (microns) }\end{array}$ & \multicolumn{1}{|c|}{ Unit } & $\begin{array}{c}\text { Fracture Aperture } \\
\text { (microns) }\end{array}$ \\
\hline \hline TCw & 181 & PPw & 103 \\
\hline PTn & 206 & PPnw & 179 \\
\hline TSw & 180 & vitrophyre & 192 \\
\hline CHn & 179 & & \\
\hline
\end{tabular}

\subsubsection{Fracture Aperture and Permeability}

Once fracture frequency and fracture porosity are determined, fracture aperture can be calculated using equation 3-2. A continuum fracture permeability was calculated using the following equation:

$$
k=\frac{F_{f} b^{3}}{12}
$$

where $\mathrm{k}=$ fracture permeability $\left(\mathrm{m}^{2}\right)$

$\mathrm{b}=$ fracture aperture $(\mathrm{m})$

$F_{f}=$ Fracture frequency $(1 / \mathrm{m})$

\subsubsection{Characteristic Curve Parameters}

The revised Young-LaPlace equation is used to calculate the van Genuchten $\alpha$ parameter from the fracture aperture. The Young-Laplace equation is:

$$
\Psi_{\mathrm{c}}=\frac{\tau \cos \theta}{\rho \mathrm{g}}\left(\frac{1}{\mathrm{r}_{1}}+\frac{1}{\mathrm{r}_{2}}\right)
$$

where $\quad \Psi_{\mathrm{c}}=$ capillary pressure head $(\mathrm{m})$

$\tau=$ surface tension $\left(0.072 \mathrm{~N} / \mathrm{m}\right.$ at $\left.20^{\circ} \mathrm{C}\right)$

$\theta=$ contact angle of the fluid onto the liquid (assumed to be 0 degrees)

$\rho=$ density of the fluid $\left(998 \mathrm{~kg} / \mathrm{m}^{3}\right.$ at $\left.20^{\circ} \mathrm{C}\right)$

$\mathrm{g}=$ acceleration due to gravity $\left(9.80 \mathrm{~m} / \mathrm{s}^{2}\right)$

$r_{1}=$ radius of the fluid meniscus in one direction (m)

$r_{2}=$ radius of the fluid meniscus in the direction perpendicular to $r_{1}(m)$ 
As we assume planar fractures, $\mathrm{r}_{2}$ goes to infinity. Knowing that the fracture aperture (b) $=2 \mathrm{r}$ and the capillary pressure head is inversely proportional to $\alpha$ :

$$
\alpha=\frac{1}{\psi_{c}}
$$

the following equation can be deduced to solve for $\alpha$ from fracture aperture:

$$
\alpha=\frac{b \rho g}{2 \tau \cos \theta}
$$

A constant value of 3.0 was used for fracture $\beta$. This number is based on the mean from a uniform distribution between the theoretical minimum and maximum $\beta$ discussed in Wilson et al. (1994).

As with the matrix characteristic curve parameters, "average" fracture characteristic curves are determined for the fractures for illustrative purposes. The mean fracture frequency for each hydrogeological unit is used to perform the calculations described above. The characteristic curves are presented in Figure 3-15. Examination of these "average" characteristic curves indicate that lateral diversion should not be expected in the fracture domain as the characteristic curves of contacting units do not intersect. Because the actual model domain is heterogeneous, there most likely are some exceptions.

\subsubsection{Fracture Network Dispersivity}

An estimate of dispersivity in fracture networks in welded units was derived from theoretical considerations for use in particle tracking simulations in the SZ. Stochastic theory of flow in heterogeneous media indicates that asymptotic macrodispersivity will be achieved after flow in the system has encountered a range of permeability values. The effective longitudinal dispersivity can be calculated from the following relationship (Gelhar and Axness, 1983):

$$
\mathrm{A}=\frac{\sigma_{\operatorname{lnk}}^{2} \lambda}{\gamma^{2}}
$$

where $\sigma_{\text {Ink }}^{2}$ is the variance of $\ln$ hydraulic conductivity (or permeability), $\lambda$ is the correlation length of conductivity, and $\gamma^{2}$ is a flow term which is approximately 1 for isotropic media. There are several assumptions with this relationship, including statistical stationarity of the hydraulic conductivity field, isotropic hydraulic conductivity, an exponential model of spatial correlation, and lognormal distribution of conductivity. It has been estimated that a travel distance of 10 to 100 times the asymptotic dispersivity is required to reach asymptotic dispersive behavior (Gelhar et al., 1979). 


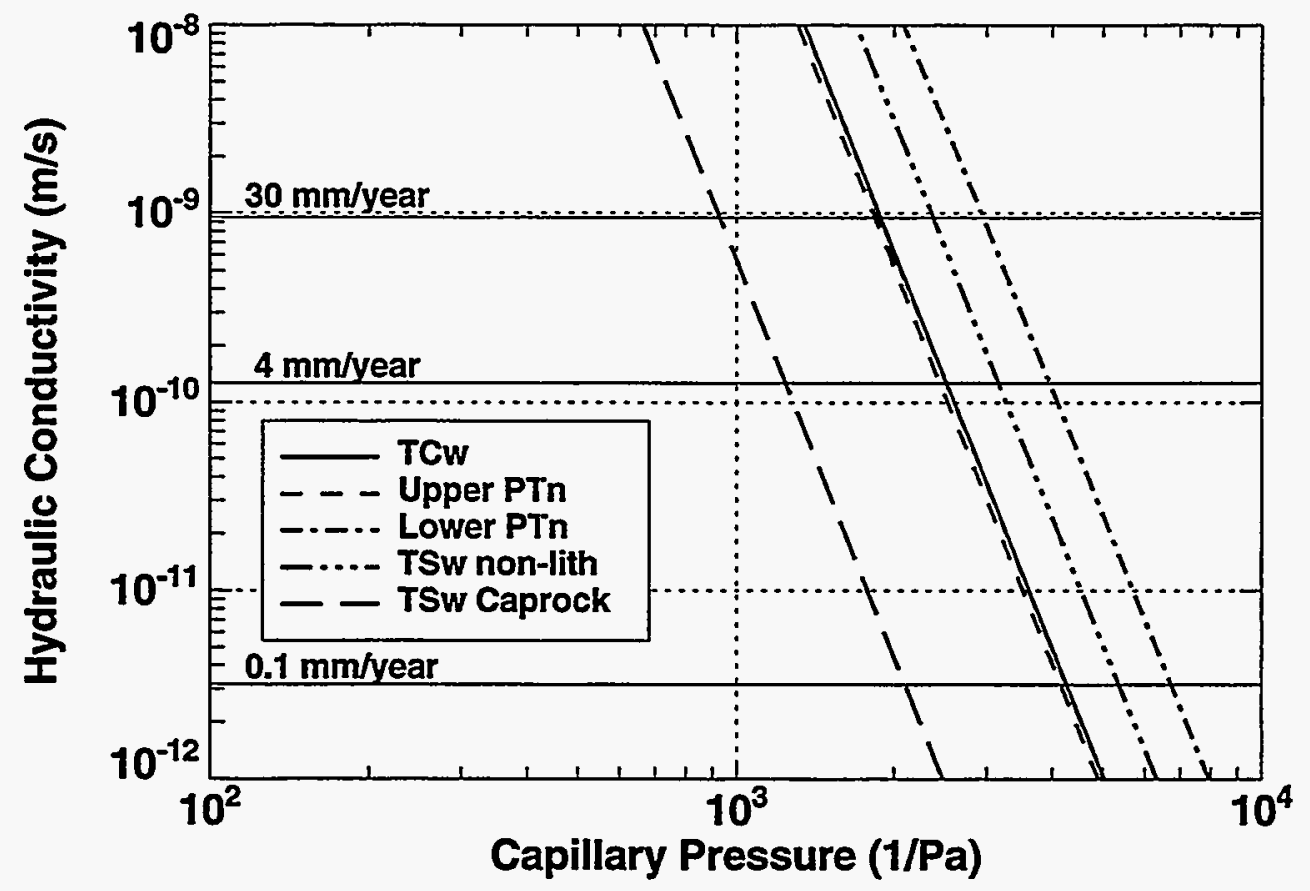

Figure 3-15 Unsaturated characteristic curves for the TCw, PTn and TSw using mean fracture van Genuchten parameters used in GWTT-95 calculations. Note that the curves of units that are in contact with each other do not intersect indicating that lateral diversion would not occur in the fracture domain above or below the PTn in a system with homogeneous units.

Air permeability data from three boreholes (UZ-16, NRG-6, and NRG-7) were used to determine the spatial continuity and variance of air permeability in the Topopah Spring welded unit $\left(\mathrm{TSw}^{19}\right)$. These in situ pneumatic tests are assumed to have measured principally the fracture network permeability (LeCain and Walker, 1994). A probability plot and experimental variogram of air permeability are shown for UZ-16 in Figure 3-16. The approximately linear probability plot of $\log$ permeability indicates a lognormal distribution as required by the theoretical expression in equation (3-7). The variogram shows a range of approximately $30 \mathrm{~m}$ for a spherical variogram model. This value corresponds to a correlation length of $10 \mathrm{~m}$ for the exponential variogram model specified in the theoretical relationship in equation (3-7). It should be noted that this value for the correlation length is a rough approximation. Variograms for the other boreholes are less distinct, but are not inconsistent with the same value of spatial correlation.

19. LeCain, G., written communications, 1995. 

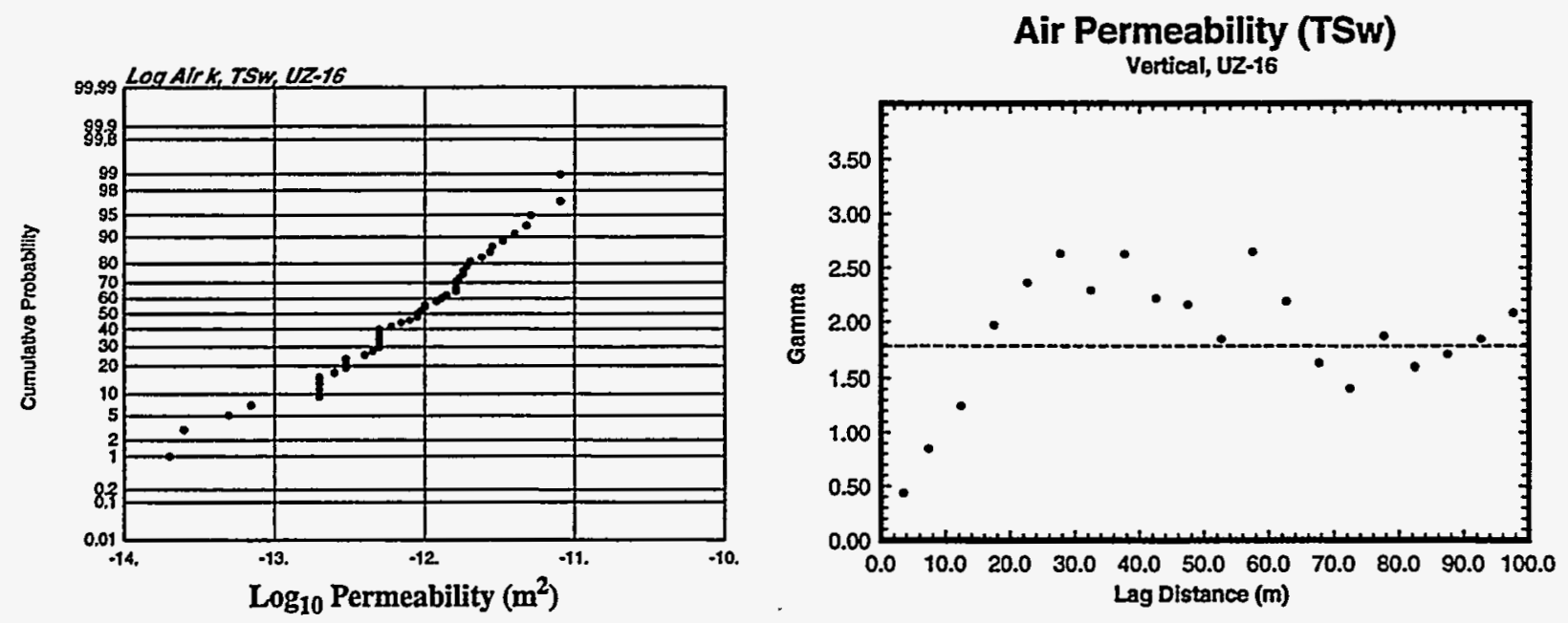

Figure 3-16 Log probability plot and variogram of air permeability measurements in borehole UZ-16.

Using the variance of log transformed permeability and the value of correlation length estimated from the variograms, an estimate of the asymptotic macrodispersivity can be calculated. The results for the three boreholes are presented in Table 3-8. These values of dispersivity generally fall within the range of experimental results (Gelhar et al., 1985).

Table 3-8: Parameters derived from air permeability data.

\begin{tabular}{|l|c|c|c|}
\hline \multicolumn{1}{|c|}{ Borehole } & $\sigma_{\text {Ink }}^{2}$ & $\lambda(\mathrm{m})$ & $\mathrm{A}(\mathrm{m})$ \\
\hline \hline NRG-6 & 1.57 & 10 & 16 \\
\hline NRG-7 & 0.75 & 10 & 7.5 \\
\hline UZ-16 & 1.78 & 10 & 18 \\
\hline
\end{tabular}

Use of the asymptotic macrodispersivity in modeling groundwater flow is only valid for flow distances greater than the minimum required to attain asymptotic behavior. Using the "rule of thumb" of 10 to 100 times the macrodispersivity for this minimum travel distance yields a range of $75 \mathrm{~m}$ to $1800 \mathrm{~m}$. This indicates that use of the asymptotic dispersivity is probably valid for modeling flow in the saturated zone for GWTT (travel distance of at least $5 \mathrm{~km}$ ). Flow in fracture networks in the unsaturated zone may not, however, reach asymptotic behavior within the travel distance of a few hundred meters to the water table.

It should be noted that this analysis of dispersivity does not include processes that occur at smaller and larger scales. There is variability in fracture aperture, on the scale of a single fracture, which leads to additional dispersion of flow. Since the air permeability measurements interrogate 
a relatively large volume of rock, there is variability in the permeability field on smaller scales which is not measured and causes more dispersion also. The effects of discrete structural features (generally on a larger scale than the air permeability testing) on the fracture permeability field are not addressed in the stochastic approach and also cause additional dispersion.

\subsection{Geostatistical Simulation of Hydrologic Properties in the Unsaturated Zone}

\subsubsection{Link to Geologic Framework Model}

Geostatistical simulation has been employed in previous GWTT studies to develop stochastic realizations of rock properties (Arnold et al., 1995). Previous efforts at geostatistical modeling have employed a two-step approach. In the first step, the stratigraphic sequence was divided into several different rock types and the spatial location of these rock types was simulated in a two or three-dimensional domain through indicator simulation (Rautman, 1994; Robey, 1994). In the second step, hydrologic properties were simulated within the indicator representation of the geologic units. In these two-step approaches to simulation, the sparse nature of the conditioning data allowed stochastic artifacts that did not fit the conceptual model of the geology (e.g., small zones of non-welded material appearing in the middle of a known thick, welded sequence). Ad hoc post processing of these simulations allowed for correction of these artifacts, but a more objective geologic basis for creating rock property simulations was desired.

For the 1995 iteration of GWTT calculations, rock property models were required along four cross-sections bisecting the areal extent of the proposed repository (Figure 3-1). The crosssections were selected to provide a representative sampling of stratigraphy, fault conditions and infiltration rates within the area of the proposed repository. The sensitivity studies discussed later in this paper (Section 4.5) are drawn from modeling done on cross-section AA (Figure 3-1) between the water table and the topographic surface. For this reason, the following discussion uses cross-section AA as an example of rock property modeling techniques that were used for all cross-sections. The general approach to the 1995 calculations is similar to that used in previous years; however, the spatial variability of rock properties was modeled directly, rather than using the two-step approach employed previously. Additionally, a dual continuum (matrix and fracture) conceptual model of the mountain has been used in the flow modeling rather than the previously used equivalent continuum conceptual model.

Direct simulation of properties requires conditioning information in addition to measured property data from the available boreholes. This additional constraining information is provided by the geologic framework conceptual model of Yucca Mountain. For the GWTT-95 calculations, software was written which would allow direct coupling of the geostatistical simulation process with an interpretation of the geology at the site (Cromer and Rautman ${ }^{20}$ ). This software is known as the GSLIB-LYNX integration module (GLINTMOD) and it provides a link between the

20. Cromer, M.V. and C.A. Rautman (in press), Use of Stratigraphic Models as Soft Information to Constrain Stochastic Modeling of Rock Properties: Development of the GSLIB-LYNX Integration Module, SAND95-2338, Sandia National Laboratories, Albuquerque, NM. 
sequential gaussian simulation program SGSIM from the GSLIB software library (Deutsch and Journel, 1992) and the digital geologic framework modeling software $\mathbf{L Y N X}^{21}$. The link between the two software packages allows a location being simulated in the SGSIM coordinate system to access the geologic framework model and determine the expected value of the property being simulated within the specific hydrogeological unit. In the current version of GLINTMOD, information from the geologic framework model is used in the geostatistical simulation when the number of conditioning points found within the local simulation search neighborhood is less than a user-specified minimum. In these cases, the mean property value for the relevant hydrogeological unit is determined from the geologic framework model and passed back to SGSIM. This mean is then used to center the posterior cumulative distribution function (cdf) used in the Monte Carlo simulation. The geologic framework model is called often in the early stages of the simulation when there are few previously simulated nodes and then, as the simulation domain is populated, additional points are conditioned upon previously simulated nodes.

\subsubsection{Gaussian Simulation}

\subsubsection{Matrix Porosity}

In any geostatistical modeling exercise, the amount and type of spatial correlation needs to be quantified. This quantification is usually accomplished through variogram modeling. A key parameter in a variogram model is the distance (range) over which properties are spatially correlated. At this time, there has been no systematic study of the range of spatial correlation both parallel and perpendicular to stratification within different lithologic units for different rock properties at Yucca Mountain. A number of studies have examined one or two properties across units in the vertical direction. Other studies have examined horizontal correlation in a single unit. The results of these studies are summarized in Table 3-9. Table 3-9 is a very high-level summary and for more information the reader is referred to the listed references. As seen in Table 3-9, there is a high amount of variability in estimates of the spatial correlation for different properties at Yucca Mountain.

The geostatistical modeling done with GLINTMOD for GWTT-95 uses a single variogram model with a specified range and parallel vs. perpendicular to stratification anisotropy to simulate porosity across all hydrogeological units. The variogram used in GLINTMOD for the simulation of matrix porosity is a spherical model with a parallel range of $460 \mathrm{~m}$ and a perpendicular range of $4.6 \mathrm{~m}$. These values are within the wide range of values shown in Table 3-9.

Constraining the geostatistical simulations with geologic information through the GLINTMOD routine requires other considerations related to the range of correlation. The geologic framework model at each cross-section contains gently dipping strata with undulations in the contacts between strata (Figure 3-3 and Figure 3-4). Geostatistical theory is based on spatial correlation as measured in a straight line between two points. Stratigraphic undulations and abrupt discontinuities, such as faults with significant offset, are not modeled well through geostatistical techniques. In order to better retain these geologic features, the parallel and perpendicular

21. Lynx Geosysytems, Vancouver, B.C., Canada 
Table 3-9: Reported ranges of spatial correlation. Spherical models were used in all studies.

\begin{tabular}{|l|l|l|l|l|l|}
\hline $\begin{array}{c}\text { Lithologic } \\
\text { Unit }\end{array}$ & \multicolumn{1}{|c|}{$\begin{array}{c}\text { Parallel } \\
\text { Range (m) }\end{array}$} & $\begin{array}{l}\text { Perpendicular } \\
\text { Range (m) }\end{array}$ & Property & \multicolumn{1}{|c|}{$\begin{array}{l}\text { Sample } \\
\text { Domain }\end{array}$} & Reference \\
\hline \hline TCw (base) & $>1300$ & $5-10$ & $\begin{array}{l}\text { porosity } \\
\text { and Ksat }\end{array}$ & $\begin{array}{l}\text { transects on } \\
30 \times 1300 m \\
\text { outcrop }\end{array}$ & $\begin{array}{l}\text { Istok, et al., } \\
1994\end{array}$ \\
\hline $\begin{array}{l}\text { TCw } \\
\text { (upper) }\end{array}$ & $0.1-0.2$ & $0.1-0.2$ & $\begin{array}{l}\text { permeabil- } \\
\text { ity }\end{array}$ & $\begin{array}{l}\text { 1x1x1m } \\
\text { block }\end{array}$ & $\begin{array}{l}\text { Tidwell, } \\
1994\end{array}$ \\
\hline $\begin{array}{l}\text { TCw } \\
\text { through CH }\end{array}$ & N/A & $8-90$ & $\begin{array}{l}\text { porosity } \\
\text { and Ksat }\end{array}$ & $\begin{array}{l}5 \text { outcrop } \\
\text { transects } \\
\text { (25-315m) }\end{array}$ & $\begin{array}{l}\text { McKenna } \\
\text { and Raut- } \\
\text { man, 1995 }\end{array}$ \\
\hline CH & 900 & $60-225$ & $\begin{array}{l}\text { porosity, } \\
\text { Ksat, bulk } \\
\text { density and } \\
\text { permeabil- } \\
\text { ity }\end{array}$ & $\begin{array}{l}\text { outcrop } \\
\text { transects } \\
\text { and drill } \\
\text { holes }\end{array}$ & $\begin{array}{l}\text { Rautman, } \\
1991\end{array}$ \\
\hline $\begin{array}{l}\text { TCw } \\
\text { through BF }\end{array}$ & $2000-5000$ & $100-250$ & porosity & $\begin{array}{l}\text { borehole } \\
\text { data (verti- } \\
\text { cal); model- } \\
\text { ing study } \\
\text { (horizontal) }\end{array}$ & $\begin{array}{l}\text { Rautman, } \\
1995\end{array}$ \\
\hline
\end{tabular}

correlation ranges are set to the low side of the values shown in Table 3-9. The relatively short parallel range and the corresponding limits on the search neighborhood require the geostatistical simulator to obtain data from the geologic framework model on a more frequent basis than if the ranges were longer. The more frequent references to the geologic framework model cause the simulations to be more tightly constrained to the features in it.

Figures 3-17 and 3-18 show a portion of section AA along the Solitario Canyon Fault as it appears in the geologic framework model and as simulated with several different correlation lengths. The distinction between the two sides of the fault deteriorates as the spatial correlation range increases. At a range length of $2000 \mathrm{~m}$ (Figure 3-18 (b)), the vitrophyre units (dark blue) continue across the fault plane before pinching out. In the same figure, the green and orange colors representing the porosity of the Calico Hills Unit continue across to the west side of the Solitario Canyon Fault. Additionally, as the correlation range increases, the distinction between the lithophysal and non-lithophysal portions of the TSw blurs. This lack of resolution is caused by the undulations in the boundaries of these units being modeled with a large correlation length as measured along a straight-line distance. These decreases in resolution are caused by fewer references to the geologic framework model during the simulations. The relative numbers of 


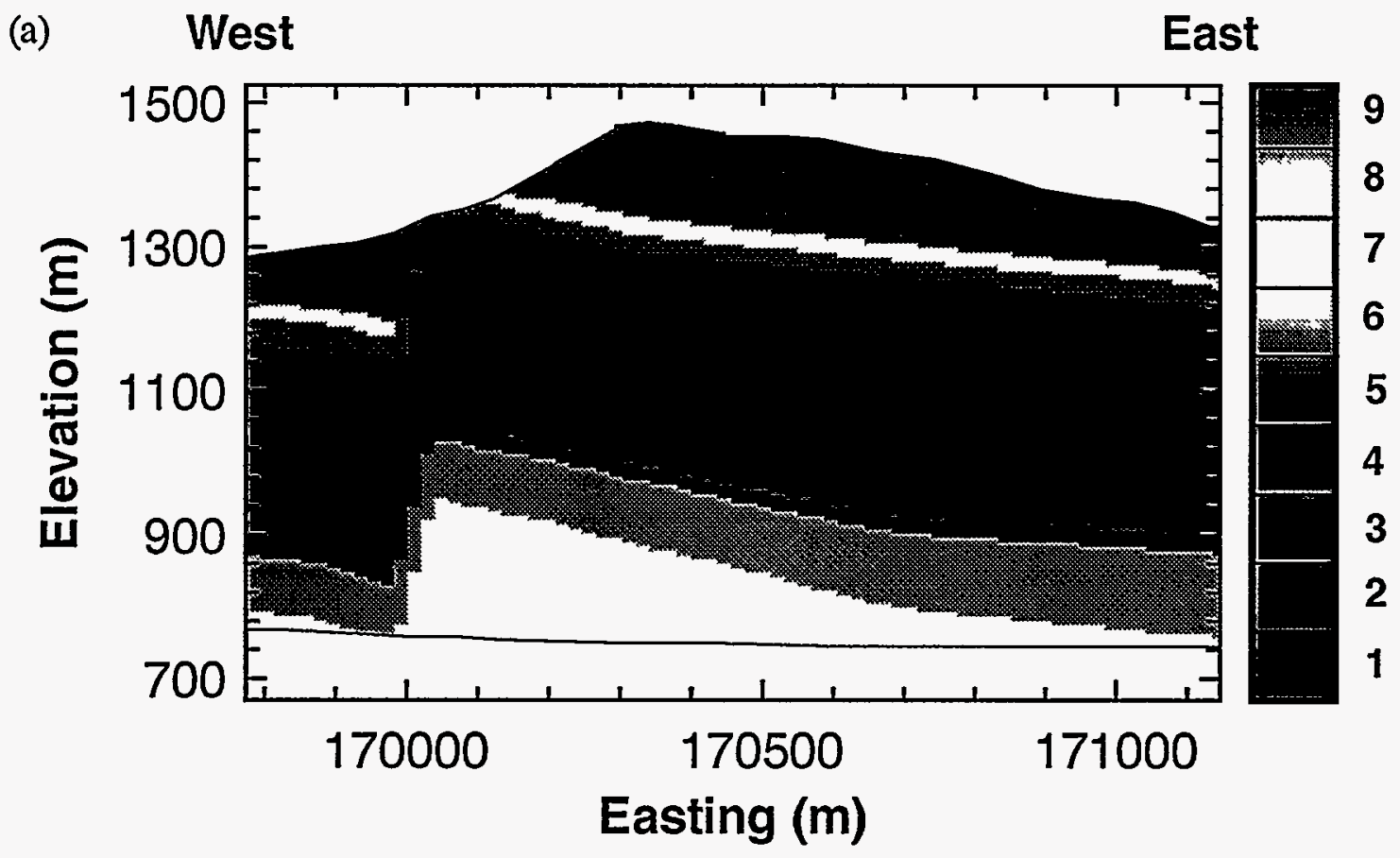

(b)

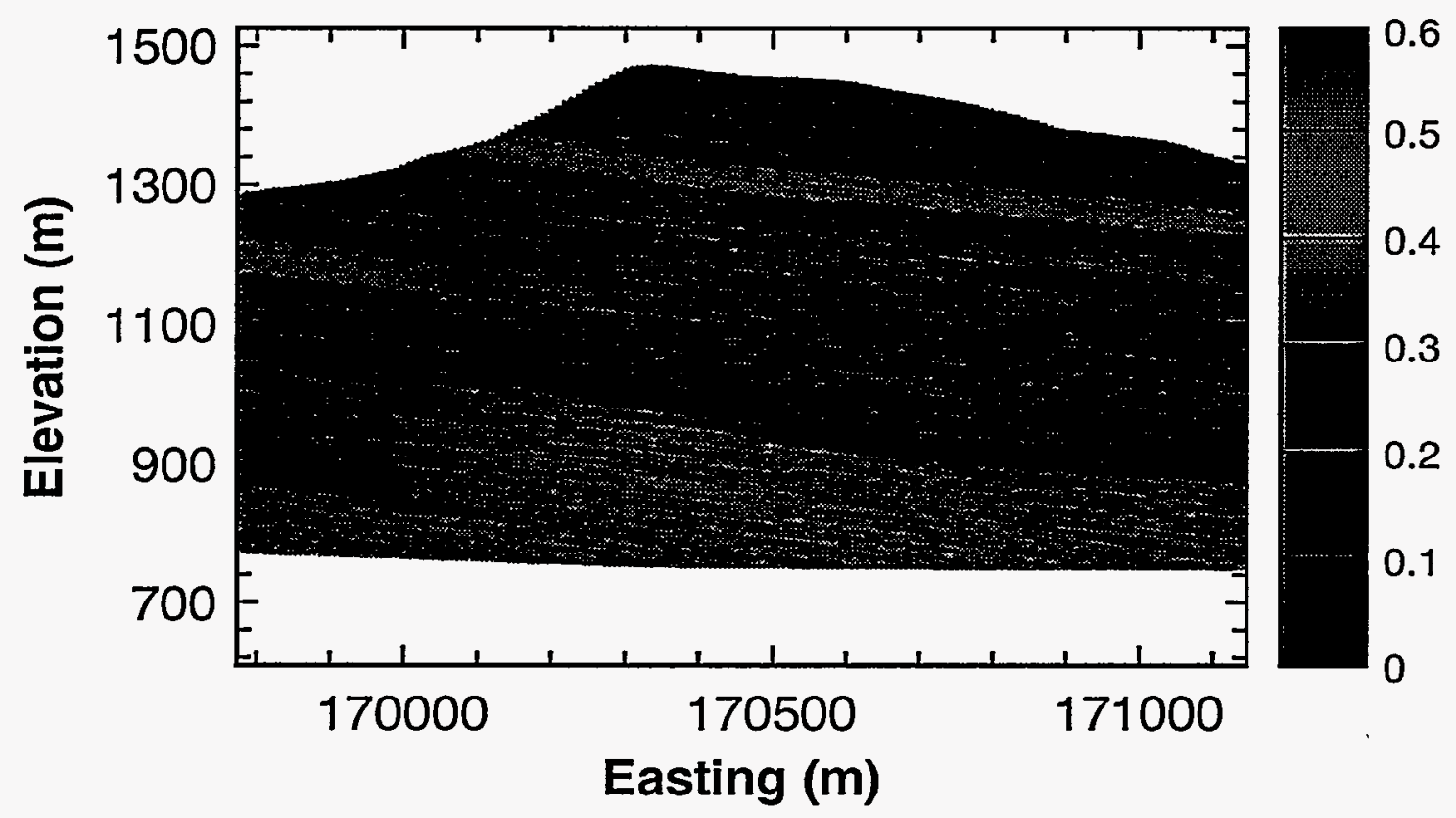

Figure 3-17 Resolution of the Solitario Canyon Fault at different ranges of spatial correlation. The fault as modeled in the deterministic framework model is shown in (a). Geostatistical simulation of porosity using GLINTMOD using a range of $460 \mathrm{~m}$ (b). The perpendicular to parallel anisotropy ratio is held at 0.01 for all realizations. 
(a)

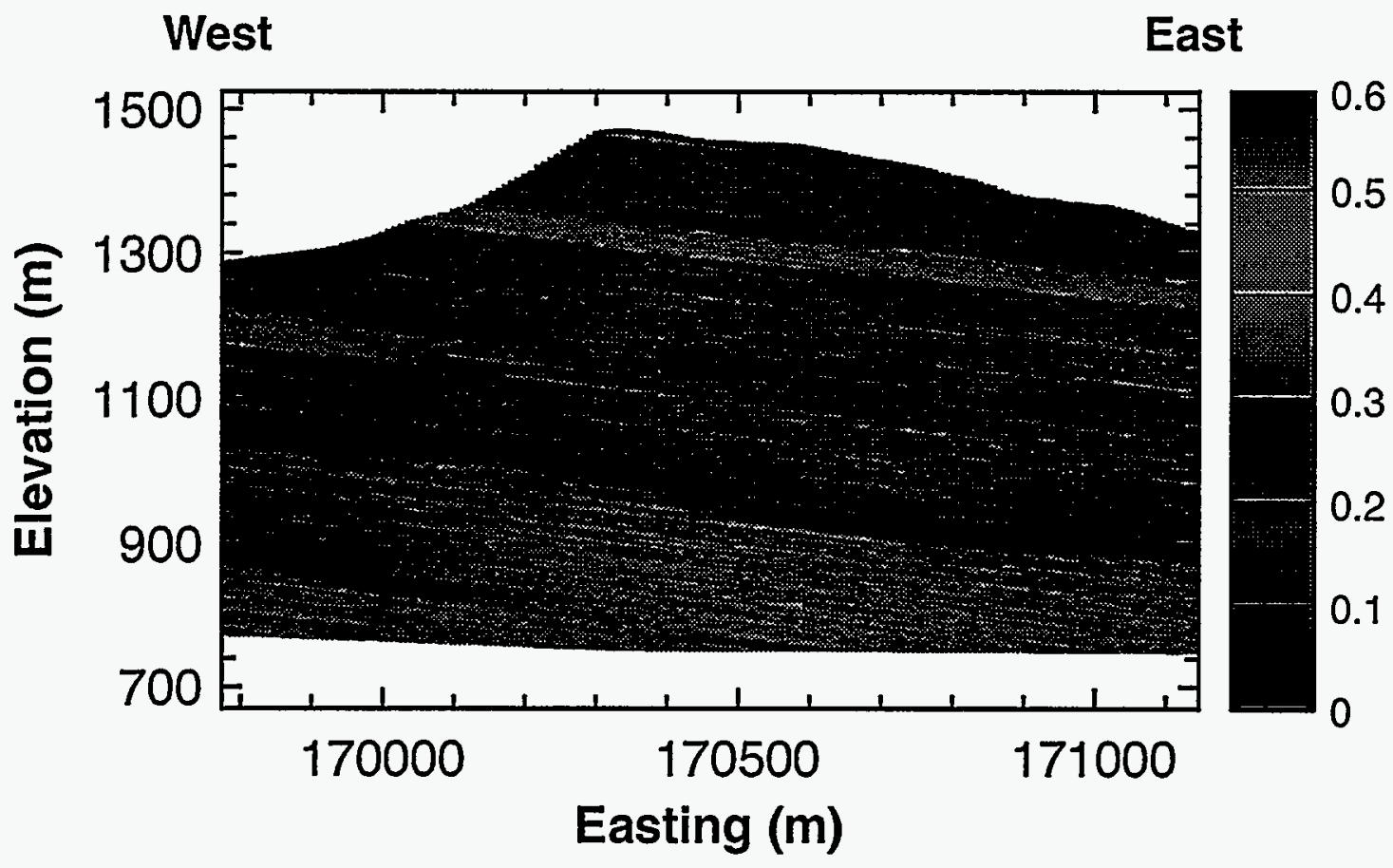

(b)

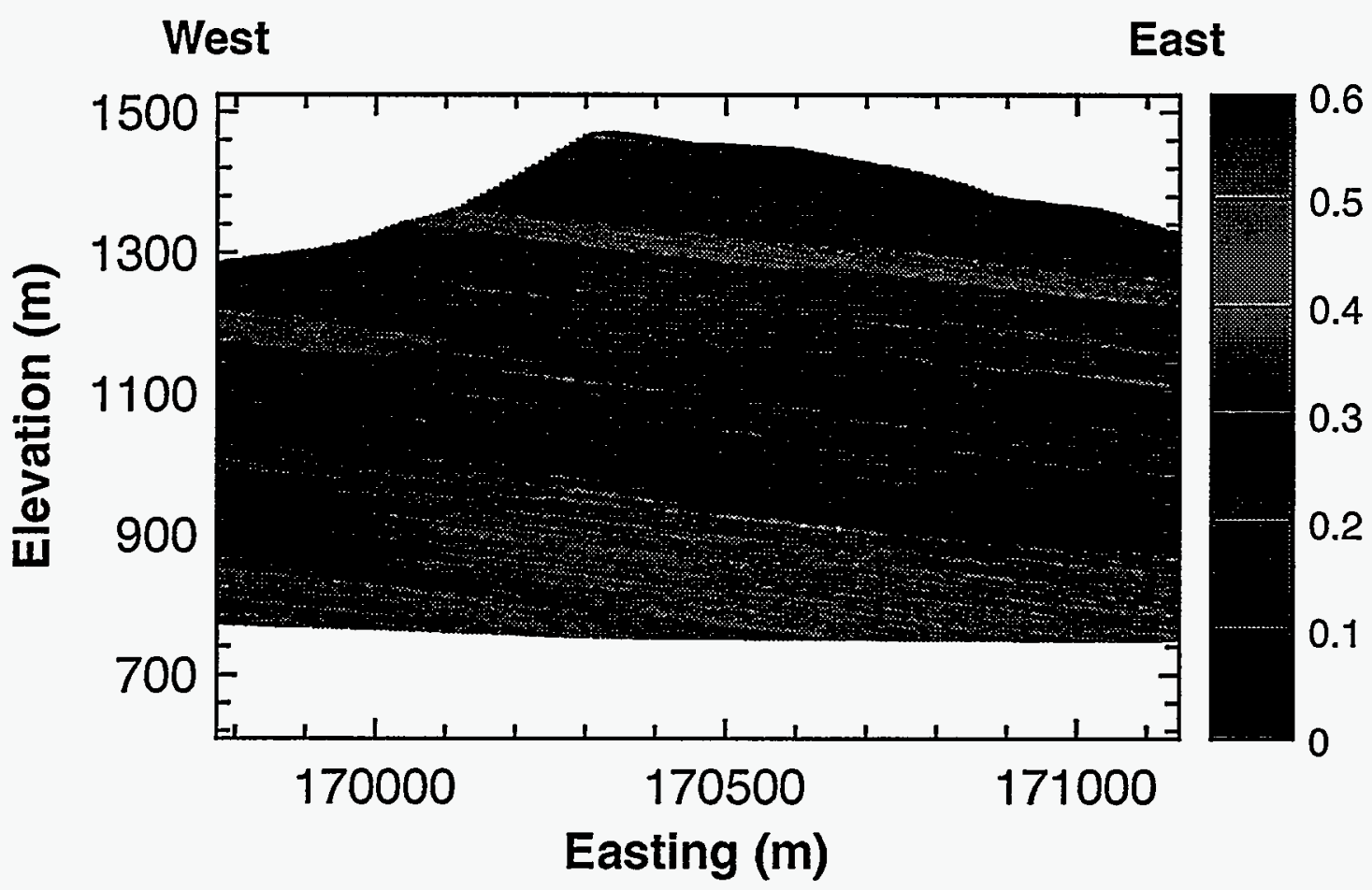

Figure 3-18 Resolution of the Solitario Canyon Fault at different ranges of spatial correlation. Porosity simulations created with a range $=1000 \mathrm{~m}$ (a) and $2000 \mathrm{~m}$ (b). The perpendicular to parallel anisotropy ratio is held at 0.01 for all realizations. 
references to the geologic framework model for the different correlation lengths are shown in Figure 3-19. As seen in Figure 3-19, even with a range of $460 \mathrm{~m}$, the number of calls to the framework model is less than five percent of the total number of simulated points.

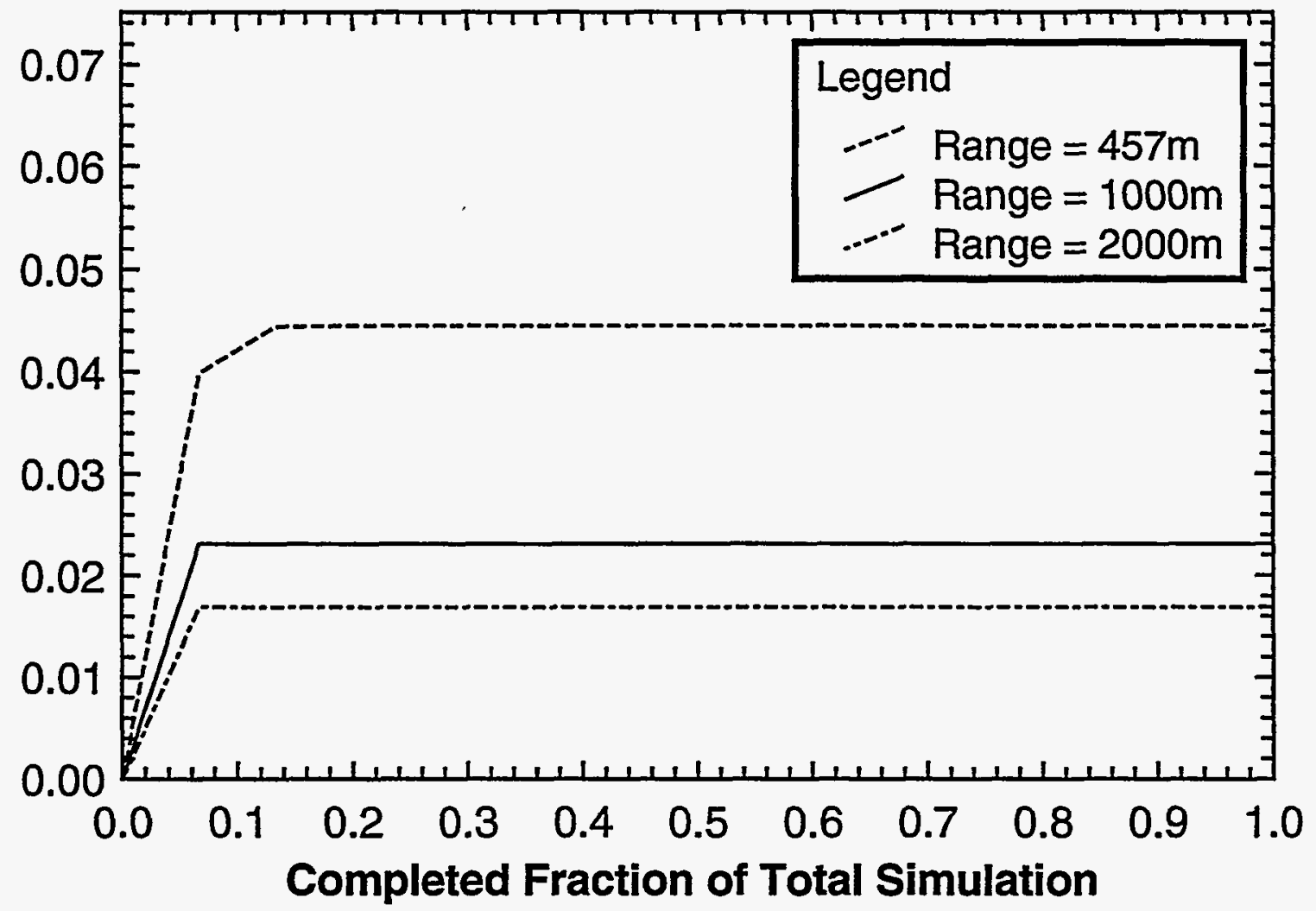

Figure 3-19 The relative number of references to the geologic framework model for simulation of porosity along section AA corresponding to the images in Figures 3-17 and 3-18.

At this time, GLINTMOD uses a separate mean porosity value for each hydrogeological unit in the domain and a single standard deviation of porosity for all units. The use of a single level of variability for all units is reasonable given the similarity of the standard deviations shown in Table 3-1. The value of the standard deviation is chosen with respect to how the simulation of porosity values is accomplished in normal-score space. The porosity conditioning data across all hydrogeological units (Figure 3-20) are transformed from their original values to a standard 
normal distribution using a normal-score transform (Deutsch and Journel, 1992). The new distribution with a mean of zero and a variance of 1.0 , is a transform of all conditioning data across hydrogeological units.

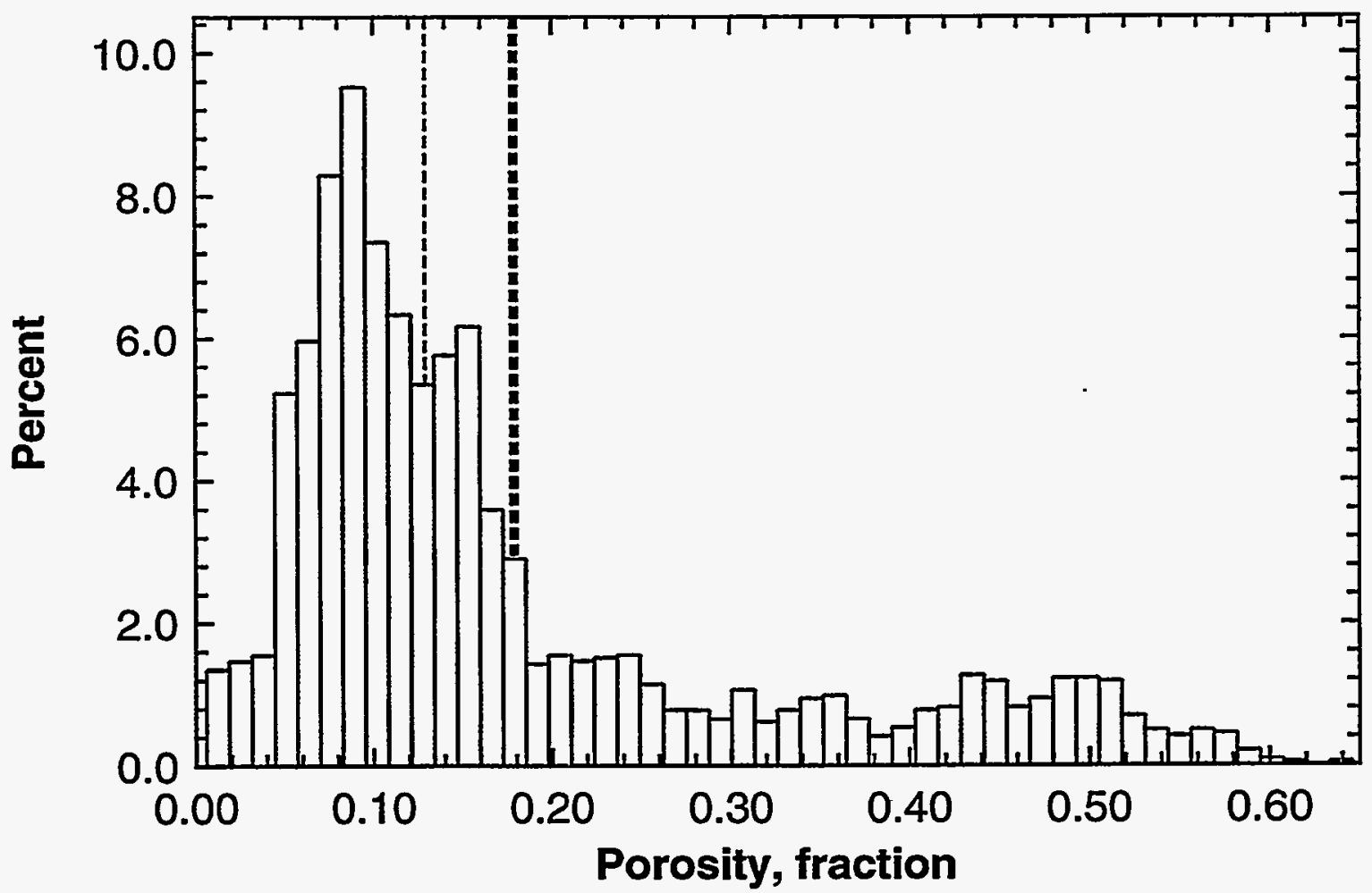

Figure 3-20 Distribution of the matrix porosity conditioning data. The thick dashed line denotes the mean and the thin dashed line denotes the median.

The overall standard deviation of the porosity conditioning data is 0.139 . From Table $3-1$, the standard deviations of each hydrogeological unit range from 0.04 to 0.10 or from $29 \%$ to $72 \%$ of the global standard deviation. On average, the standard deviation of any hydrogeological unit is $44 \%$ of the global standard deviation (0.06) and this value is used in GLINTMOD for the simulation of porosity in all units.

Matrix porosity simulations (Figure 3-21) are created using porosity data from boreholes shown in Figure 3-1 and summarized in Table 3-1 as conditioning data and the deterministic interpretation of the geology along each cross-section (e.g., Figure 3-3). The discretization of the geostatistical simulation domain varies between cross-sections and is described in Table 3-10. 


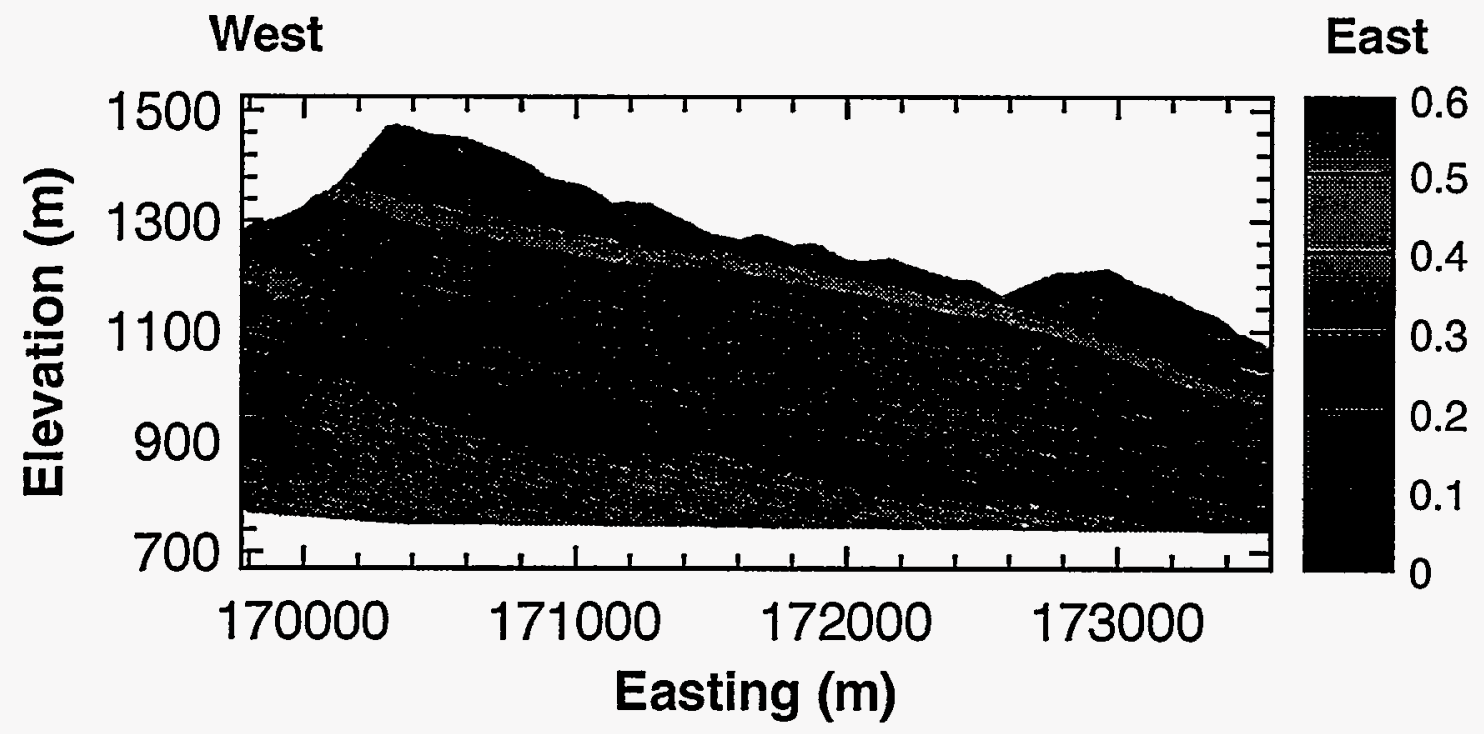

Figure 3-21 Realization number one of porosity along cross-section AA.

Table 3-10: Discretization of cross-sections for geostatistical simulation.

\begin{tabular}{|c|c|c|c|c|c|}
\hline $\begin{array}{c}\text { Cross- } \\
\text { Section }\end{array}$ & $\mathrm{nx}$ & $\begin{array}{c}\text { delta-x } \\
(\mathrm{m})\end{array}$ & $\mathrm{nz}$ & $\begin{array}{c}\text { delta-z } \\
(\mathrm{m})\end{array}$ & total $\mathrm{n}$ \\
\hline \hline $\mathrm{AA}$ & 414 & 9.14 & 1000 & 0.91 & 414,000 \\
\hline $\mathrm{BB}$ & 440 & 9.14 & 1000 & 0.91 & 440,000 \\
\hline $\mathrm{CC}$ & 400 & 13.72 & 778 & 1.37 & 311,200 \\
\hline $\mathrm{DD}$ & 330 & 13.72 & 778 & 1.37 & 256,740 \\
\hline
\end{tabular}

\subsubsection{Coregionalization of Matrix Saturated Hydraulic Conductivity}

Linear coregionalization is a process by which geostatistical simulations of correlated rock properties can be created. This technique is felt to be well suited to the data available at Yucca Mountain and is employed for the generation of saturated hydraulic conductivity from porosity fields for GWTT-95 calculations. 
The ultimate goal of the simulation process in this study is to generate geostatistical realizations of multiple properties which honor the univariate statistics for each property, the covariances between properties and the spatial statistics of each property as well as the spatial cross-covariances between properties. One way to accomplish this goal is cosimulation (Deutsch and Journel, 1992). Cosimulation requires the statistical moments of each property, the variogram of each property and the cross-variogram between all pairs of properties as input. Additionally, co-simulation requires that one property be oversampled with respect to another and that the samples of the two properties be collocated. This presents a problem at Yucca Mountain, as there are often not enough data of a given property to adequately define a variogram, let alone a crossvariogram between that property and another.

Previous attempts to generate realizations of multiple properties at Yucca Mountain have relied upon geostatistically simulating a primary property (usually porosity) and using that as a surrogate for secondary properties (e.g., saturated hydraulic conductivity). The secondary property was obtained from the primary property simulation by applying the appropriate regression relationship to each simulated location of the primary property. This approach has produced unsatisfactory results when the regression relationship contains a large error component (a relatively low coefficient of determination, $\mathrm{R}^{2}$ ). The large error component in the regression relationship produces a spatially random, uncorrelated field of the secondary variable superimposed upon the primary variable field. Ignoring the error term in the regression implies an unrealistic 1:1 correlation between the properties.

The technique described herein, linear coregionalization, lies somewhere between true cosimulation, where the information contained on the primary variable informs and constrains the simulation of the secondary variable, and the previously applied regression techniques. Linear coregionalization provides a means of reproducing the observed, or assumed, variograms, crossvariograms and regression relationships between properties in multiple simulations.

The mathematics behind linear coregionalization are described in Journel and Huijbregts (1978) and Luster (1985). The following description is similar to that found in Luster (1985) and is given for two correlated properties. However, there is no theoretical limit on the number of correlated properties that can be simulated. Two basic constructs are at the basis of coregionalization. The first is the variance-covariance matrix from traditional statistics:

$$
\left[\begin{array}{ll}
\mathrm{C}_{11} & \mathrm{C}_{12} \\
\mathrm{C}_{21} & \mathrm{C}_{22}
\end{array}\right]
$$

where $C$ denotes variance and the subscripts refer to.two different properties, $Z_{1}$ and $Z_{2}$. The final goal of coregionalization is to produce realizations of the two properties which honor the variancecovariance matrix as derived from the available data. If the distributions are relatively normal to begin with, it is possible to scale them to standard normal distributions. After this scaling, the diagonal terms in the variance-covariance matrix are 1.0 and the off diagonals are now equal to the correlation coefficient between the two properties. The second construct is the relation between covariance and the correlation coefficient between the two properties: 


$$
r=\frac{C_{12}}{\sqrt{C_{11} C_{22}}}
$$

The correlation coefficient, $r$, can be obtained from the regression relationship between the properties and the variances of each property are obtained from the data.

Spatial correlation of each property and of the combination of the properties can be represented as:

$$
\begin{aligned}
\gamma_{1}(\mathrm{~h}) & =\mathrm{CO}_{1}+\mathrm{Cl}_{1} \mathrm{Sph}_{\mathrm{a}}(\mathrm{h}) \\
\gamma_{2}(\mathrm{~h}) & =\mathrm{CO}_{2}+\mathrm{Cl}_{2} \mathrm{Sph}_{\mathrm{a}}(\mathrm{h}) \\
\gamma_{12}(\mathrm{~h}) & =\mathrm{CO}_{12}+\mathrm{Cl}_{12} \mathrm{Sph}_{\mathrm{a}}(\mathrm{h})
\end{aligned}
$$

where $C O$ denotes the nugget, $C l$ is the sill and $S p h_{a}$ refers to a spherical semivariogram model with a range of $a$ and $C O+C I=1.0$. The subscripts refer to two different properties being modeled. The spherical model is chosen here as an example; other valid, positive definite semivariogram models could also be used.

The objective now is to obtain a simulation of both property $Z_{1}$ and $Z_{2}$ that fits the variogram models specified above. To do this, two new variables are defined: $Y_{1}$ and $Y_{2}$. Both of these variables have zero mean and covariance functions $K_{1}(h)$ and $K_{2}(h)$ and they are independent of each other (i.e., $K_{12}(h)=0$ for all $h$ ). Linear combinations of these two new variables can be used to define the original variables:

$$
\begin{aligned}
& Z_{1}(x)=a_{11} Y_{1}(x)+a_{12} Y_{2}(x) \\
& Z_{2}(x)=a_{21} Y_{1}(x)+a_{22} Y_{2}(x)
\end{aligned}
$$

Because the two $Y$ variables are independent, the covariance functions of the $Z$ s are given as:

$$
\begin{gathered}
\mathrm{C}_{1}(\mathrm{~h})=\mathrm{a}^{2}{ }_{11} \mathrm{~K}_{1}(\mathrm{~h})+\mathrm{a}^{2}{ }_{12} \mathrm{~K}_{2}(\mathrm{~h}) \\
\mathrm{C}_{2}(\mathrm{~h})=\mathrm{a}^{2}{ }_{21} \mathrm{~K}_{1}(\mathrm{~h})+\mathrm{a}^{2}{ }_{22} \mathrm{~K}_{2}(\mathrm{~h}) \\
\mathrm{C}_{12}(\mathrm{~h})=\mathrm{a}_{11} \mathrm{a}_{21} \mathrm{~K}_{1}(\mathrm{~h})+\mathrm{a}_{12} \mathrm{a}_{22} \mathrm{~K}_{2}(\mathrm{~h})
\end{gathered}
$$

These equations provide a means by which two independent realizations of $Y_{1}$ and $Y_{2}$ can be linearly combined through the coefficients of the $A$ matrix to provide realizations of $Z_{1}$ and $Z_{2}$ which satisfy the variance-covariance matrix as described above.

The solution for the coefficients of the $\mathbf{A}$ matrix is non-unique, as there are only three equations for the known values $C_{1}, C_{2}$ and $C_{12}$. One of the coefficients is usually set to zero (Luster, 1985) which sets one of the $Z$ 's equal to one of the $Y$ 's: 


$$
\begin{gathered}
Z_{1}(x)=Y_{1}(x) \\
Z_{2}(x)=a_{21} Y_{1}(x)+a_{22} Y_{2}(x)
\end{gathered}
$$

The final variances and covariances of the $Z$ variables are given as:

$$
\begin{gathered}
\mathrm{C}_{1}(\mathrm{~h})=\mathrm{K}_{1}(\mathrm{~h}) \\
\mathrm{C}_{2}(\mathrm{~h})=\mathrm{a}^{2}{ }_{21} \mathrm{~K}_{1}(\mathrm{~h}) \mathrm{a}^{2}{ }_{22} \mathrm{~K}_{2}(\mathrm{~h}) \\
\mathrm{C}_{12}(\mathrm{~h})=\mathrm{a}_{21} \mathrm{~K}_{1}(\mathrm{~h})
\end{gathered}
$$

The above expressions for $Z_{1}(x)$ and $Z_{2}(x)$ in terms of $Y_{1}(x)$ and $Y_{2}(x)$ are one possible linear model for the coregionalization of $Z_{1}(x)$ and $Z_{2}(x)$.

The model of linear coregionalization produces realizations of multiple properties that honor the regression relationship between any two properties. At Yucca Mountain, there are different regression relationships between properties within different portions of the mountain. An obvious example is the different relationship between matrix porosity and saturated hydraulic conductivity within the zeolitized portion of the mountain as compared to the non-zeolitized portion of the mountain. These different relationships are shown in Figure 3-6. In order to honor these different relationships, simulated locations within the zeolitized portion of each crosssection do not use the linear coregionalization method, but are modeled with the regression relationship derived from the field data (Figure 3-6). This relationship is modeled with the linear regression equation:

$$
\text { Ksat }=b_{0}+b_{1}(\phi)+\varepsilon
$$

where $b_{0}$ is the intercept, $b_{1}$ is the slope and $\varepsilon$ are normally distributed errors about the regression line modeled in accordance with the observed coefficient of determination.

Rather than producing two, independent, unconditional realizations which can be transformed into the appropriate properties, one realization (porosity) is conditional to the available data including the framework model as described above. This conditioning of one property follows the approach taken in a previous unsaturated zone study (Desbarats, 1995). A second realization is created to be transformed into the Ksat values. This realization is standard normal (mean $=0$, std. dev. $=1$ ) and unconditional and is shown in Figure 3-22. The realization shown in Figure 3-22 was made with the same variogram parameters as the realizations in Figure 3-21, but with a different seed for the random number generator and without any conditioning data.

These two realizations now need to be combined through the linear coregionalization process to produce realizations of porosity and Ksat which honor the observed correlation between the two properties. After the realizations have been transformed (in this case the porosity realization remains the same by choosing $a_{12}$ equal to zero), they are converted from standard 
This page intentionally left blank.

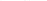




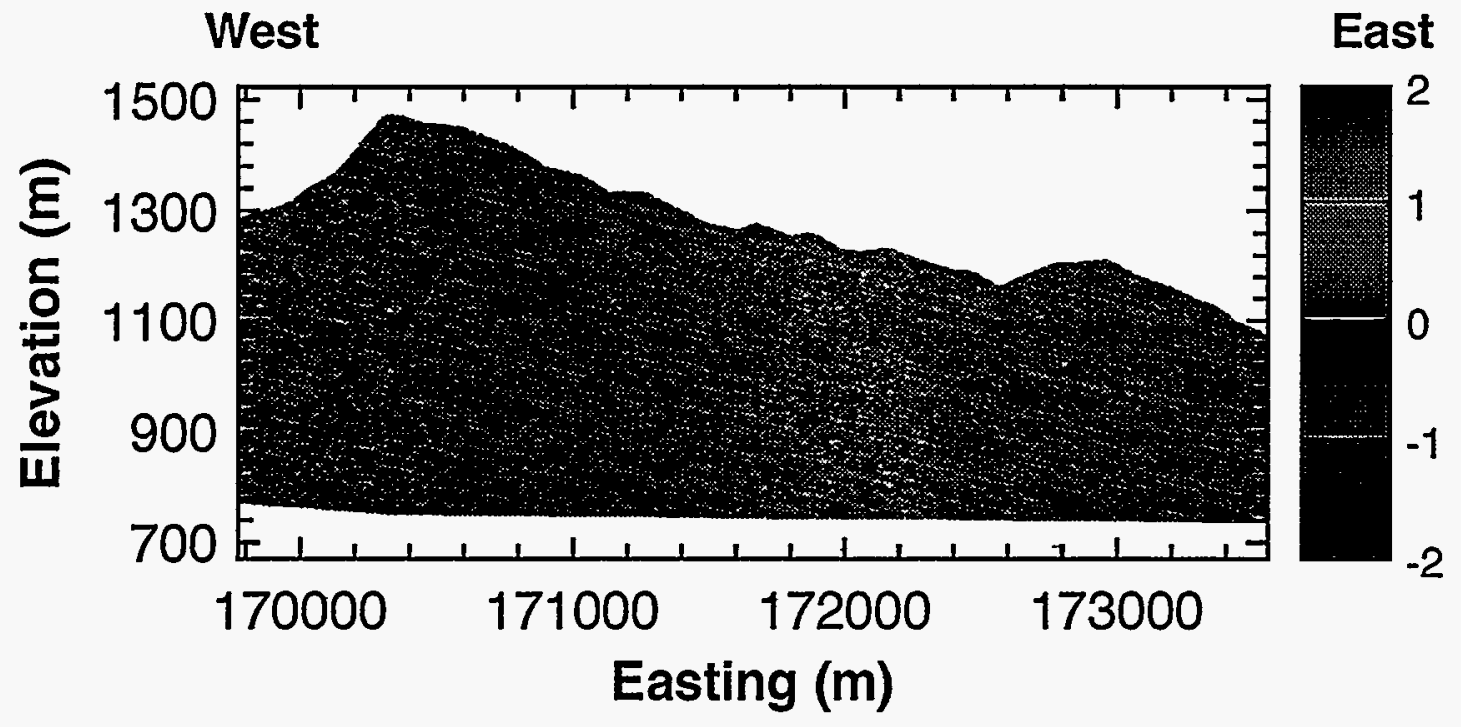

Figure 3-22 Unconditional, standard-normal realization number 1 at cross-section AA used in the coregionalization process.

normal back to the appropriate mean and variance by multiplying each value in the realization by the specified standard deviation and adding the mean. The resulting coregionalized realizations are shown in Figure 3-21 (porosity has remained unchanged) and Figure 3-23.

The linear coregionalization model shown here offers an efficient way to generate correlated fields of parameters which all retain the specified variogram and the observed correlation between the two properties. This technique is valid for any number of parameters (only 2 are discussed here) and any value of correlation between parameters (high, low, positive or negative), thus it could have other applications at Yucca Mountain (e.g., thermal conductivities). Also, using the linear coregionalization model allows the use of the GLINTMOD software without any further modification to that code.

A constraint on the linear coregionalization model is the requirement that variograms and cross-variograms of all simulated properties have the same model type, structure and range. For the case of porosity and Ksat, there are not enough Ksat data to confirm nor deny this assumption; however, it is possible to conceive of the two properties having different correlation lengths. The model of linear coregionalization does allow for each variable to have a different nugget effect. Compared to true cosimulation, linear coregionalization does not exploit the covariance by using 


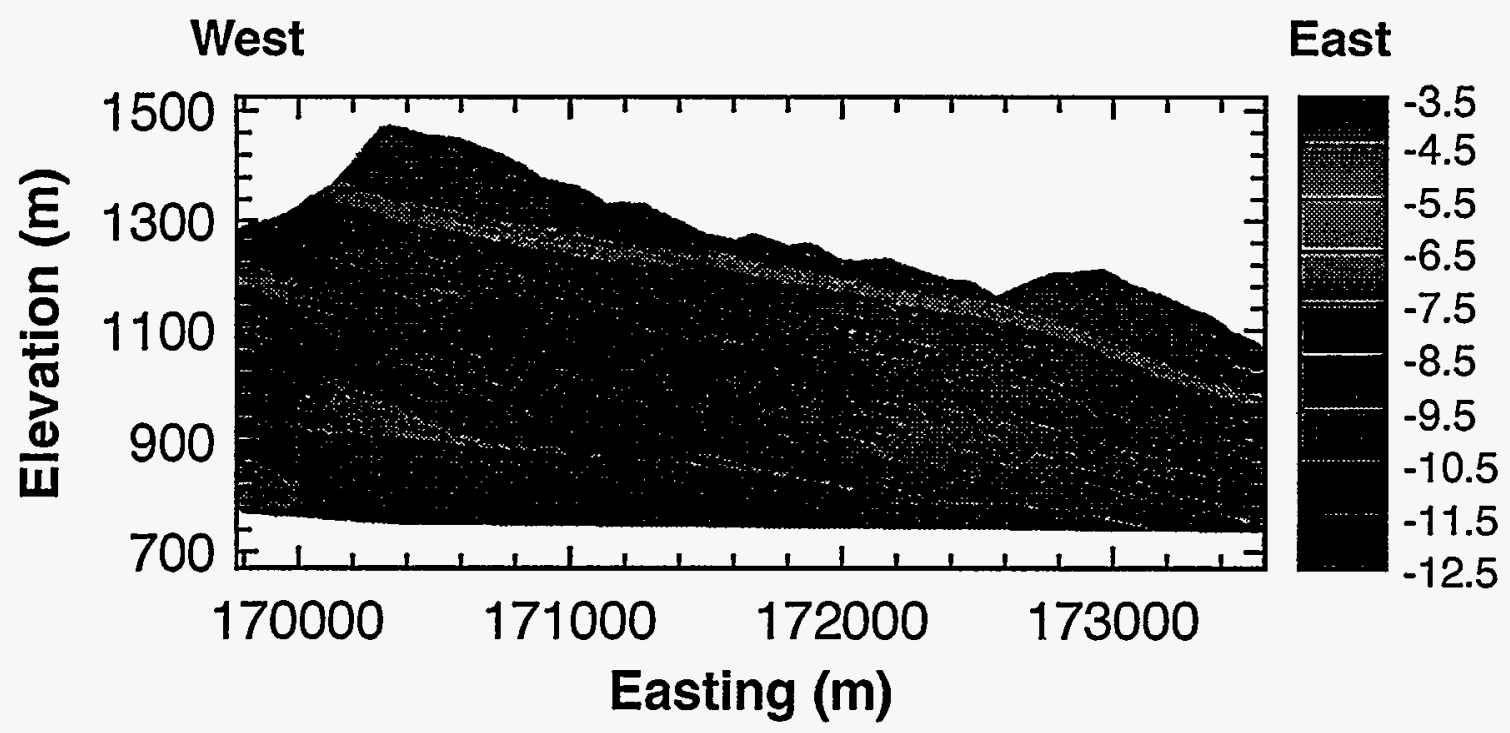

Figure 3-23 Realization number 1 of section AA for $\log _{10}$ matrix saturated hydraulic conductivity $(\mathrm{m} / \mathrm{s})$. This realization was produced through the model of linear coregionalization with porosity. Note the relatively lower (dark blue) conductivities in the zeolitized zone at the base of the section.

the oversampled parameter (porosity in this case) to inform and constrain the simulations of the other parameter(s). For cosimulating porosity and Ksat this is irrelevant at this time as there are not enough Ksat data to do true cosimulation.

\subsubsection{Fracture Frequency}

Frequency of cooling fractures are largely a function of the lithology; more welded units exhibit higher fracture frequencies. Mean values of fracture frequency for the nine hydrogeological units are given in Table 3-6. The data distribution is shown in Figure 3-24. The data represent measurements of fracture frequency from approximately 550 ten-foot-long drill cores. These measurements are adjusted to account for partial recovery of the core and fracture orientation relative to the vertical boreholes using equation 3.1 .

The simulation of fracture frequency is done in GLINTMOD using the same variogram parameters and discretization as used for simulating matrix porosity. The only exception is that several of the units have a non-zero probability that a ten foot long core would be unfractured. These probabilities are also shown in Table 3-6. The GLINTMOD software was modified to accommodate these probabilities. The mean fracture frequency used in the simulations for each unit is taken from the values in Table 3-6 and the standard deviation is equal to the global observed fracture frequency standard deviation ( $0.45 \log$ fractures/10 feet). For every simulated 


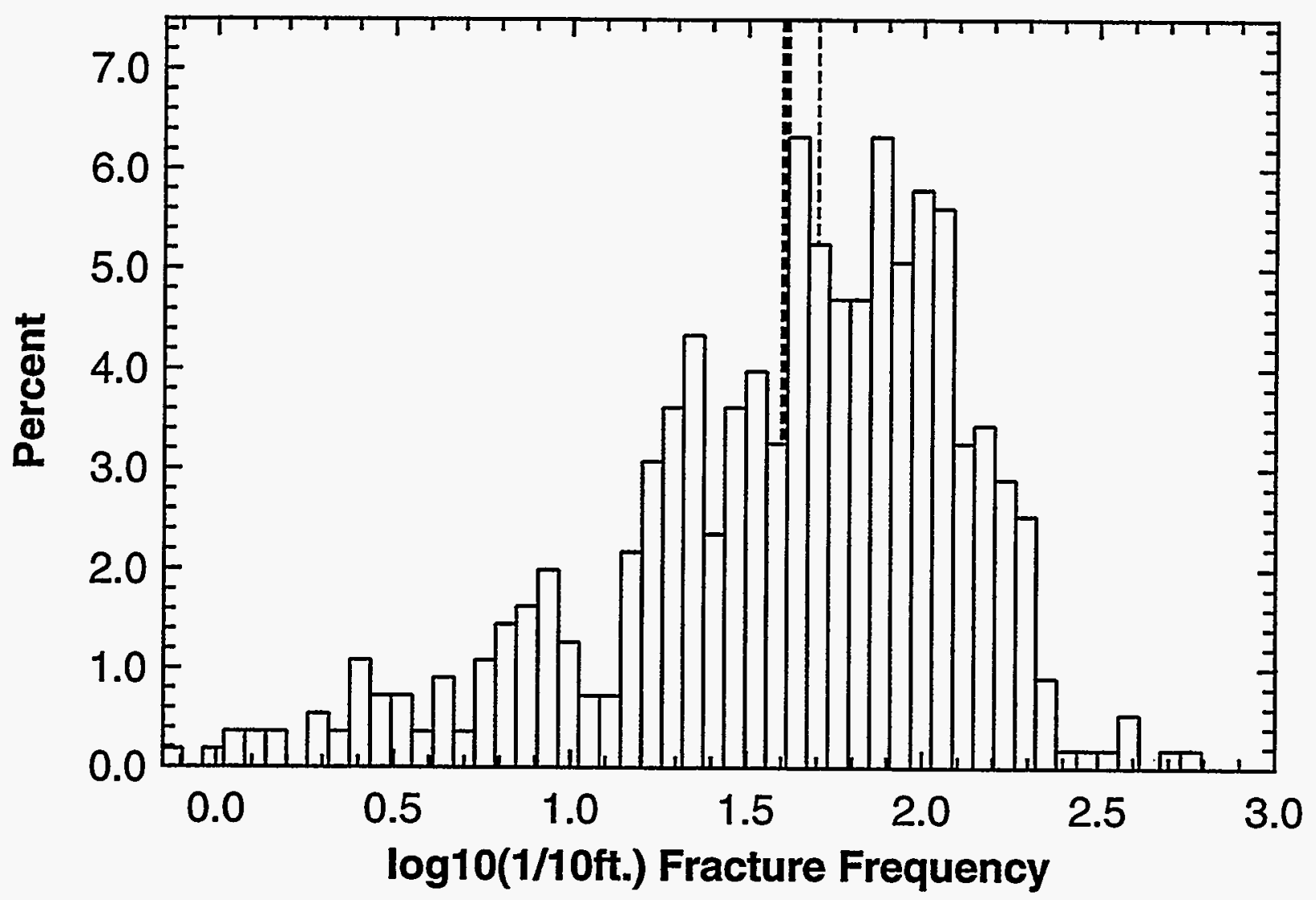

Figure 3-24 Distribution of fracture frequency conditioning data in $\log _{10}(1 / 10 \mathrm{feet})$. The thick dashed line denotes the mean value and the thin dashed line denotes the median value.

location, there is a probability that the fracture frequency ( $1 / 10$ feet) will be zero. If the random number drawn is below that probability, the fracture frequency at that location is arbitrarily set to 0.01 (100 $\mathrm{m}$ fracture spacing), otherwise the frequency is simulated using the standard GLINTMOD approach.

\subsubsection{Indicator Simulation of Tectonic Fracture Zones}

Vertical zones of relatively higher fracture frequency that cut across lithologic layering include the large scale faults that bound the potential repository block and smaller faults throughout Yucca Mountain. The locations of these zones are simulated using an indicator algorithm (SISIMPDF; Deutsch and Journel, 1992). Large scale fault zones are viewed as deterministic features and are located in the simulation domain with conditioning data (labelled fault zones in Figure 3-25). The smaller features are considered as randomly located features and are produced by the indicator simulation. The area within the higher frequency fracture zones is 
Solitario Canyon

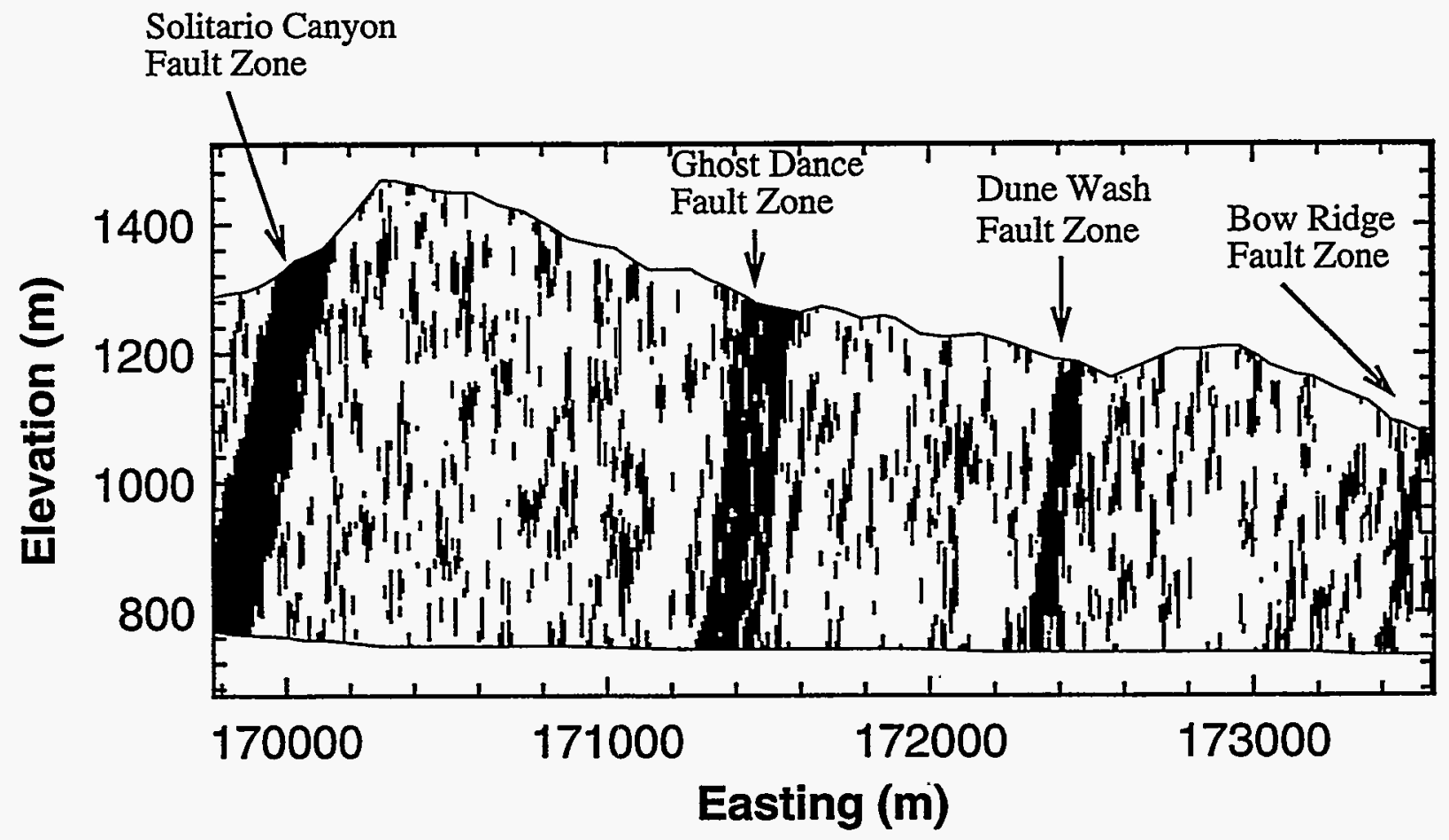

Figure 3-25 Realization number 1 at section AA of the high fracture frequency zones created with indicator geostatistical simulation. The high fracture frequency zones are black and the areas of background fracture frequency are white. The mapped, deterministic fault features are labelled.

20 percent of the total domain for cross-sections $\mathrm{AA}, \mathrm{BB}$ and $\mathrm{CC}$. The high fracture frequency zones comprise 30 percent of section DD to reflect the proximity of the cross-section to the Drill Hole Wash fault zone.

Realizations created by the two fracture models are merged to create a single representation of fracture frequency that is a combination of cooling and tectonic fractures. Field studies have shown that fracture frequency within a fault zone is sharply reduced in the high porosity, nonwelded units of the Paint Brush Group and fracture frequency is also relatively low in the Calico Hills nonwelded unit (Scott et al., 1983). These observations have led to the development of the relationship between porosity and the effect of tectonically induced fractures shown in Figure 3-26. The relationship in Figure 3-26 is used to determine a factor by which the cooling fracture frequency, or background frequency, is multiplied within the high frequency zones. Determination of the multiplication factor as a function of porosity forces the tectonic fractures to be more frequent in the welded zones and by stopping the slope in Figure 3-26 at a 


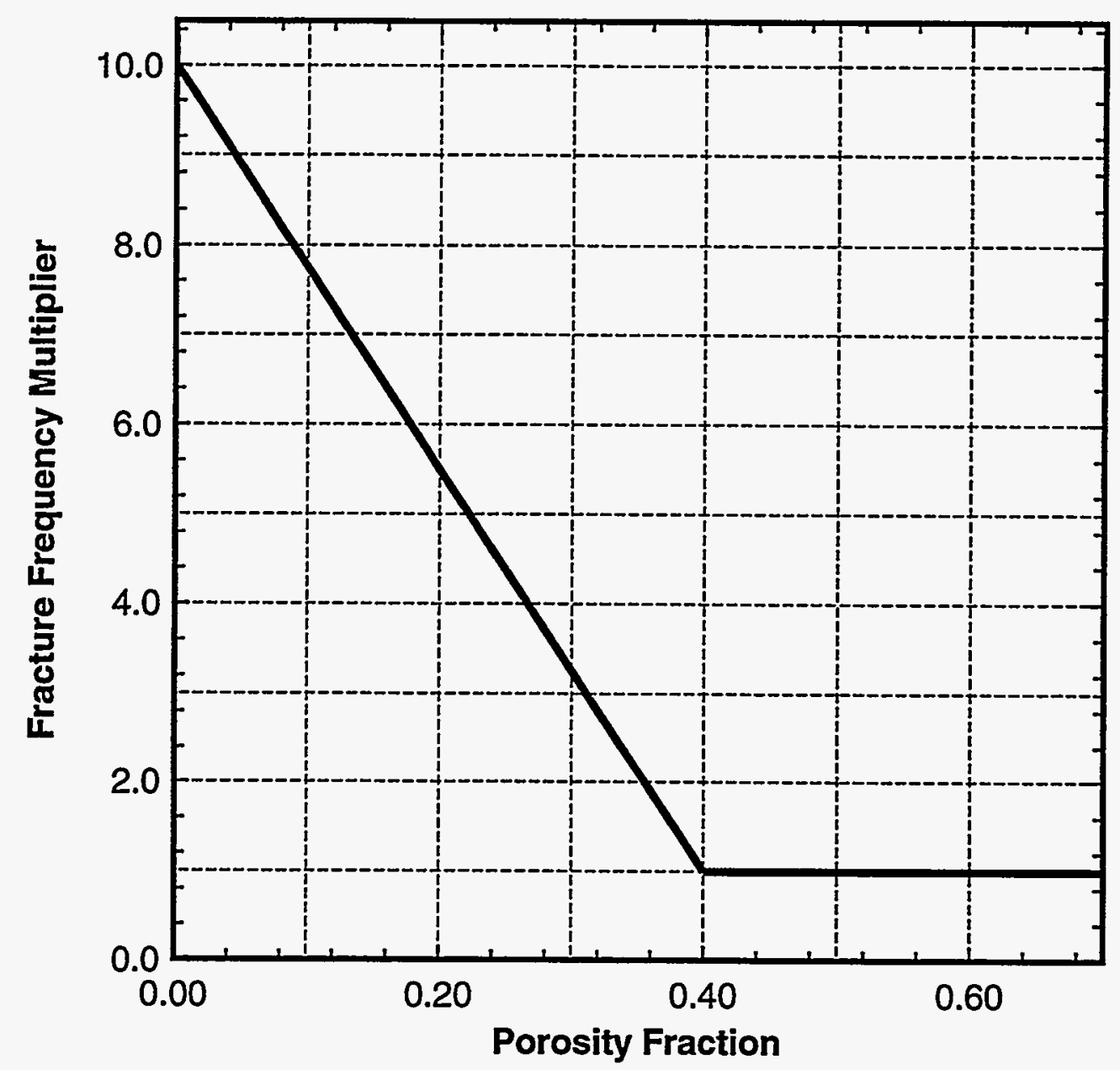

Figure 3-26 Empirically derived relationship between matrix porosity and the fracture frequency multiplier.

porosity fraction of 0.40 , the fracture frequency in nonwelded units in the Paintbrush Tuff remains unaffected by tectonic fracturing (Figure 3-27). The essentially unfractured Paintbrush Tuff nonwelded units are consistent with field observations (Scott and Castellanos, 1984).

\subsubsection{Upscaling of Hydrologic Properties}

The volume of the computational elements within TOUGH2 contain 10's to 100's of geostatistical simulation elements. The geostatistical elements have values that are representative of the properties as measured in core plugs or in 3 meter core barrel sampling of fracture frequency. In order that the simulated properties accurately represent flow and transport at the computational element scale, upscaling is required. 
This page intentionally left blank.

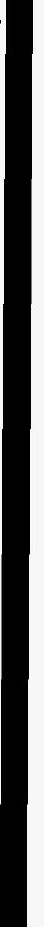




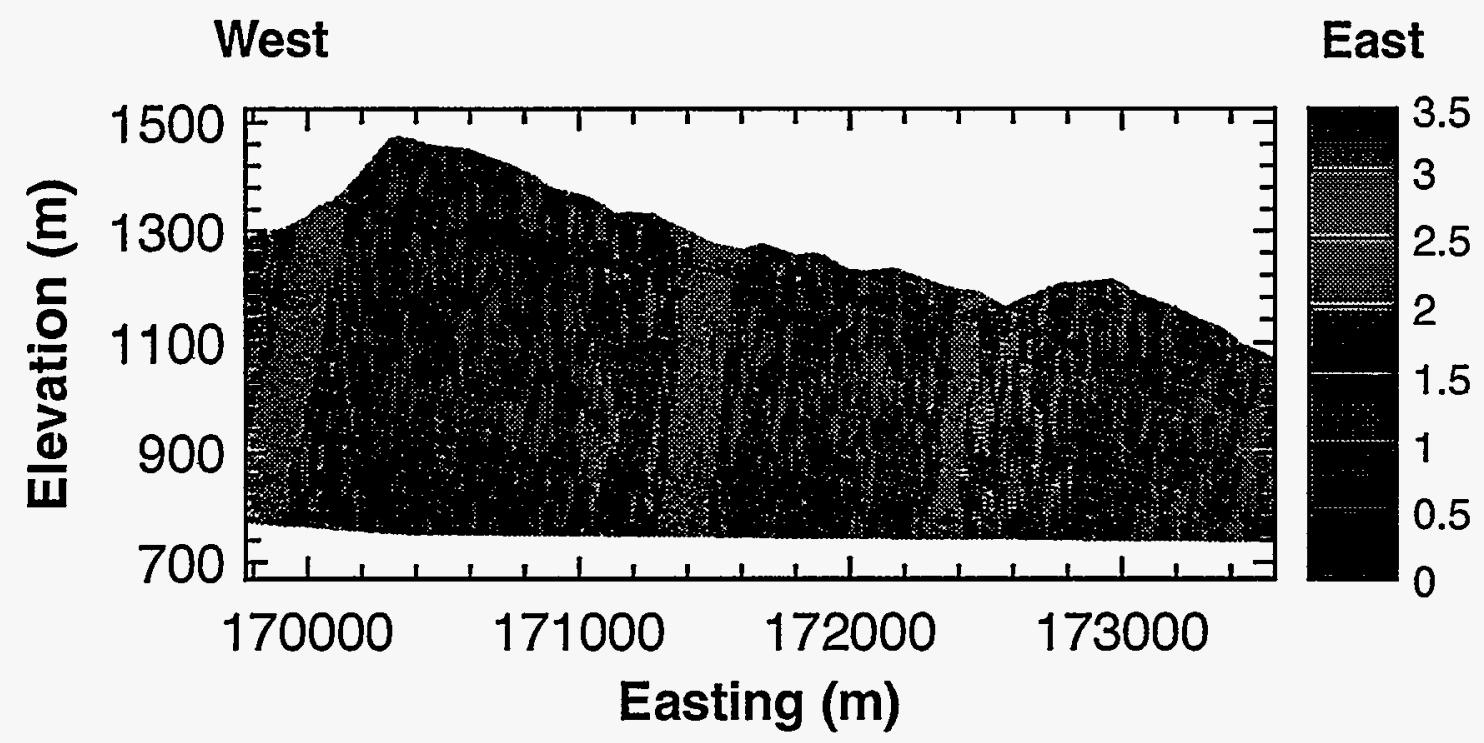

Figure 3-27 Realization number 1 at cross-section $A A$, of $\log _{10}$ (1/10feet) fracture frequency derived by combining realizations of cooling fractures with tectonically induced fracturing through the relationship defined in Figure 3-26.

Software has been written to read in geostatistical simulations of multiple properties, assuming they have been created on the same grid, along with locations of centroids for the computational grid and the polygon defining the domain of the computational grid. The properties are then upscaled to the computational grid and the input file for TOUGH2 is written for either the effective continuum or the dual permeability model.

The properties in the geostatistical simulations are upscaled to the computational grid using power-law averaging with a possible different power coefficient for each property. The power law average is defined by:

$$
\mathrm{Z}_{\mathrm{up}}=\left(\frac{1}{\mathrm{n}} \sum_{\mathrm{i}=1}^{\mathrm{n}} \mathrm{Z}_{\mathrm{i}}^{\mathrm{w}}\right)^{\frac{1}{\mathrm{w}}}
$$

where $Z$ is a variable and $n$ is the number of geostatistically simulated values falling within the boundary of the computational grid element. The power coefficient, $w$, ranges from -1.0 to 1.0. A value of -1.0 is the harmonic mean and 1.0 is the arithmetic mean. The power law equation is undefined when $w=0.0$, however through an expansion of the equation, a value of $w=0.0$ produces the geometric mean. For the GWTT-95 calculations, a power coefficient of 1.0 is used 
for matrix porosity and fracture frequency and a coefficient of -0.4 is used for saturated matrix hydraulic conductivity as determined through a series of numerical experiments (McKenna and Rautman, 1996). Power law averaging provides a fast and accurate averaging technique that is flexible enough to work with irregular grids.

The TOUGH2 computational grid for section AA is shown in Figure 3-28. The grid discretization is based on the geologic framework model for each cross-section and is designed to capture the thin units of the PTn and the basal vitrophyre of the TSw. The effect of upscaling on the property distributions is examined at cross-section AA by comparing distributions at the sample data and geostatistical simulation scales with the resulting upscaled values.

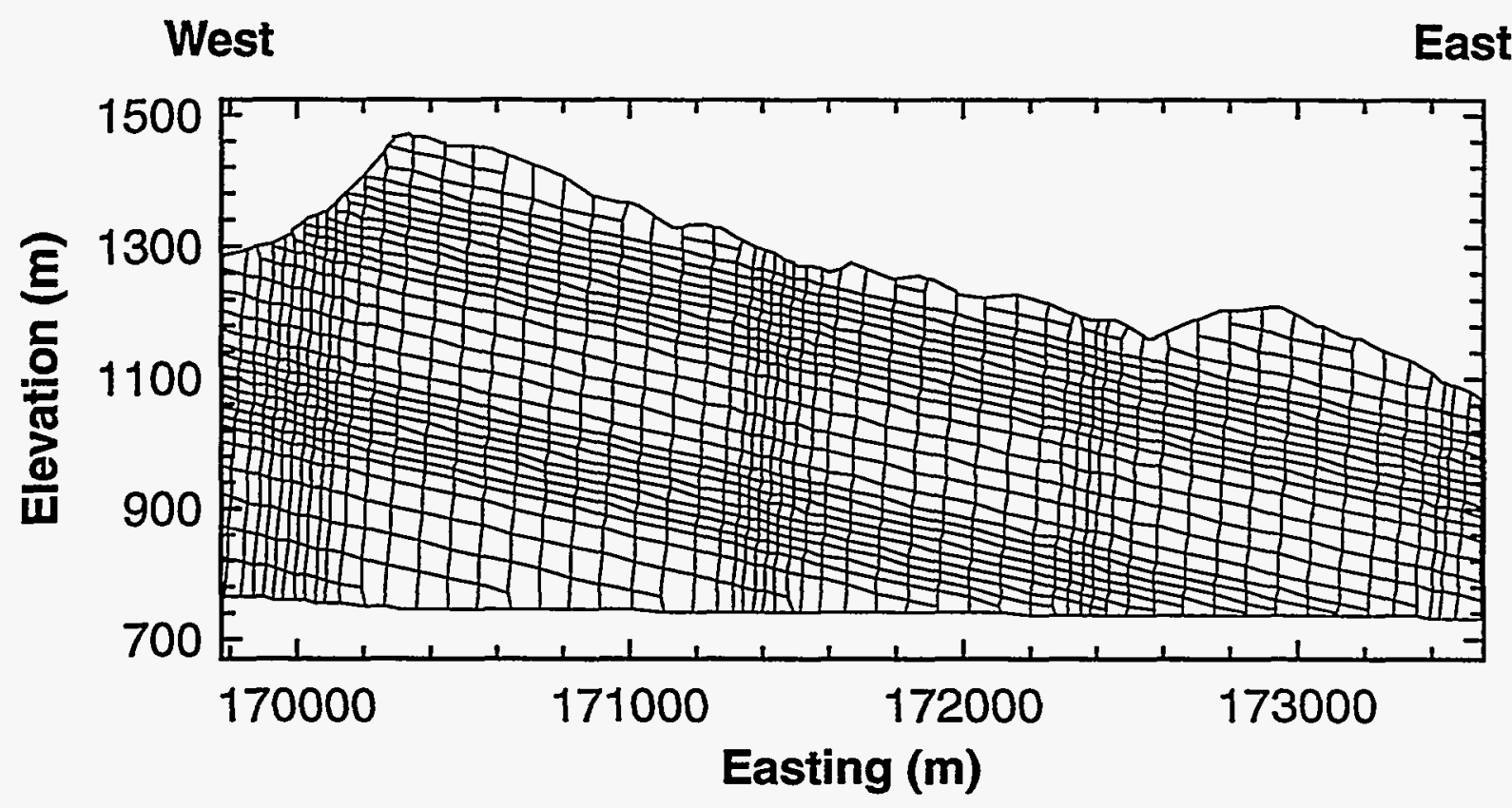

Figure 3-28 Computational grid for TOUGH2 used on cross-section AA. Properties are scaled from the geostatistical simulation scale to the computational scale.

Figure 3-29 shows the distribution of the porosity conditioning data along with the distribution of porosity for each of the 10 realizations along section AA. The averaging of geostatistically simulated values into a single value for each computational element entails a loss of information on the fine scale distribution of porosity. This loss of information is represented as a reduction in variance from the conditioning data distribution to the final upscaled distribution. The standard deviation of the porosity conditioning data is 0.139 while the standard deviation of the upscaled porosity distribution for the ten realizations averages 0.0726 , a 48 percent reduction in variability. The geometry of the hydrogeological units is retained through the scaling process as demonstrated in Figure 3-30.

Demonstrating the effects of upscaling on the distribution of Ksat is less straightforward than for porosity. Ksat values are determined stochastically for each realization through the linear coregionalization process. The simulated Ksat values are a function of the simulated porosity 


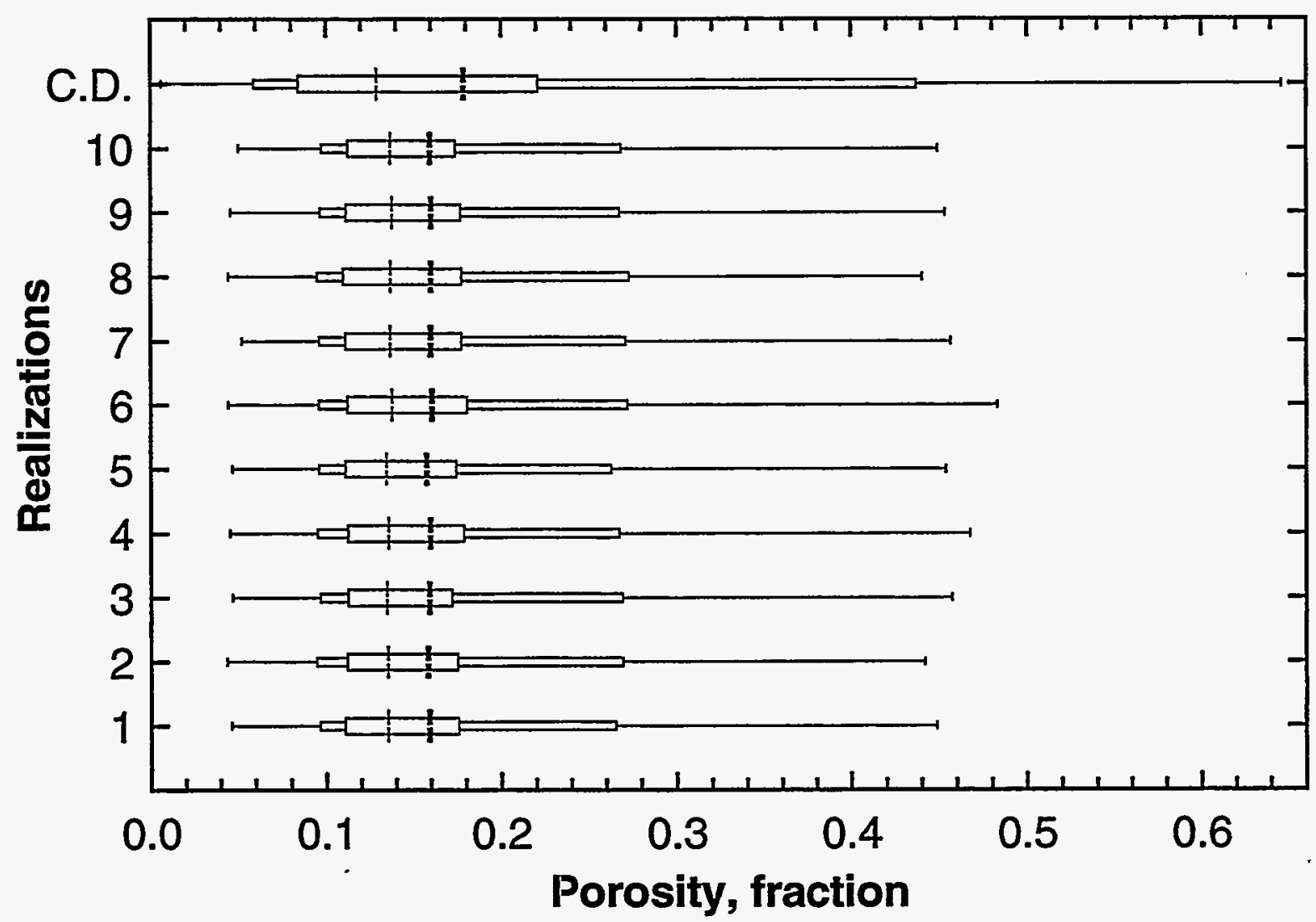

Figure 3-29 Box and whisker plots of the porosity distributions for the ten realizations after being upscaled and for the porosity conditioning data. The thick and thin dashed lines denote the mean and median respectively. The boxes define the 10th, 25th, 75th and 90th percentiles and the ends of the whiskers are the minimum and maximum values.

values and the regression relationships between the properties for both the zeolitized and nonzeolitized portions of the mountain. The effect of upscaling on the Ksat values is shown in Figure 3-31. The distribution of Ksat values for realization number one along section AA is representative of the distributions of all ten realizations and is shown prior to upscaling. The center of the Ksat distributions decreases through the upscaling process because a negative power coefficient is used in Equation 3-16. The negative power coefficient accounts for the direction of the principal-flow direction (downward) being nearly perpendicular to the stratigraphic layering.

\subsubsection{Derived Hydrologic Properties}

Properties that are not simulated are derived from the upscaled values of those properties that were simulated. In summary, the parameters that are simulated at the geostatistical scale and upscaled to the flow model scale are matrix porosity, matrix saturated hydraulic conductivity, fracture frequency without tectonic fractures, fracture frequencies with combined tectonic and 


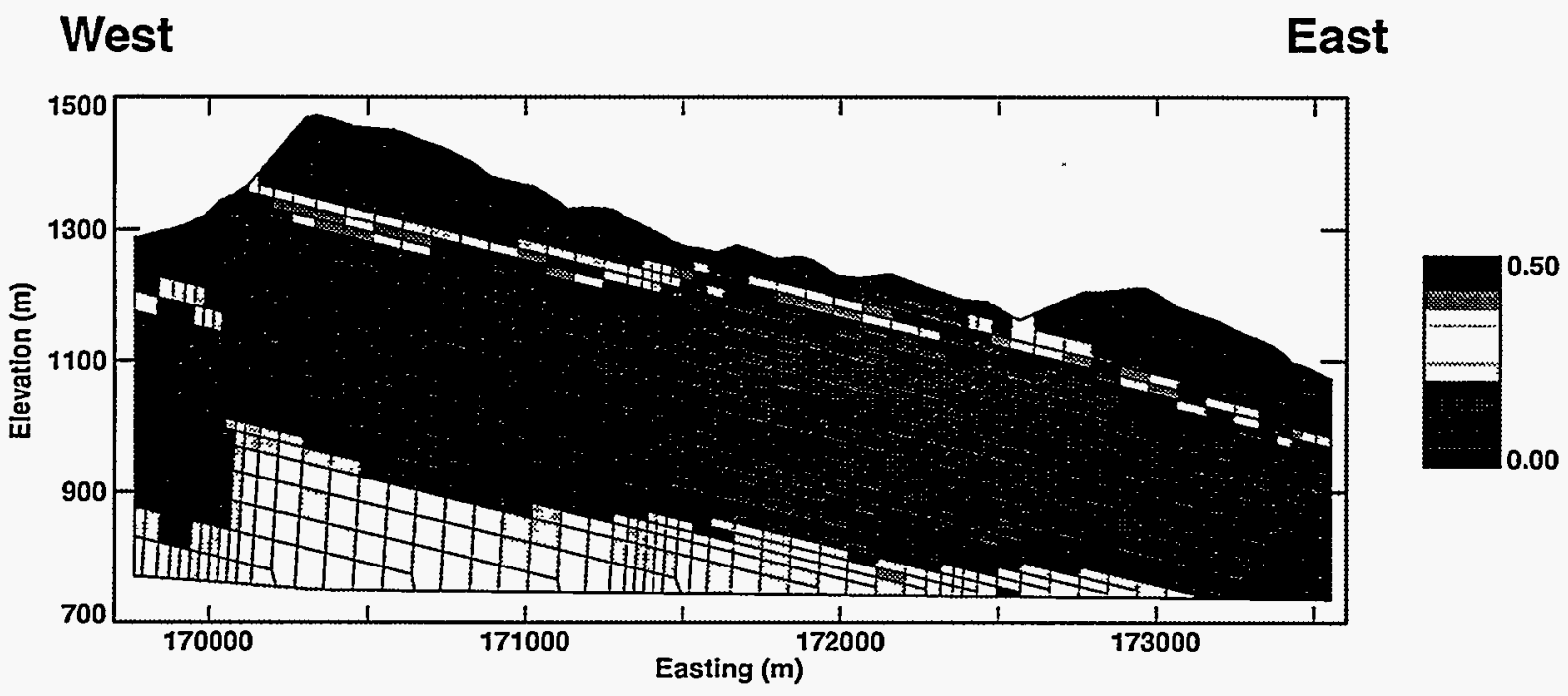

Figure 3-30 Upscaled values of porosity shown in the computational grid.

cooling fractures. Table 3-11 summarizes how the other parameters necessary for the flow model calculations are derived, either through regression or direct relationships with know parameters. It should be noted that fracture frequencies simulated before the tectonic fracture are simulated are used to calculate fracture aperture. This was done so that the fracture aperture would not be lower in the fault zones and zones of higher fracture frequency that the other zones, thus creating an artificially low fracture permeability in these zones. Matrix $\alpha$ and fracture porosity are both derived through regression relationships without modeling the error of the regression. This is justified because it is believed that the variability of the parameters due to uncertainty is represented sufficiently in the geostatistical simulations of porosity and linear coregionalization of saturated hydraulic conductivity which remain geologically viable. The values of $\beta$ for both the fractures and the matrix are set to constants as discussed in Sections 3.2.1.3 and 3.2.2.4.

The values of fracture permeability used in the flow model are plotted in Figure 3-31. A qualitative comparison of these fracture permeability values in the TSw generated by geostatistical simulation to the distribution of air permeability shown in Figure 3-16 indicates that the fracture permeabilities used in this study are about one order of magnitude higher than measured values. This discrepancy may be due to sampling bias in the air permeability measurements caused by the inability to obtain measurements in highly fractured zones of the boreholes. 


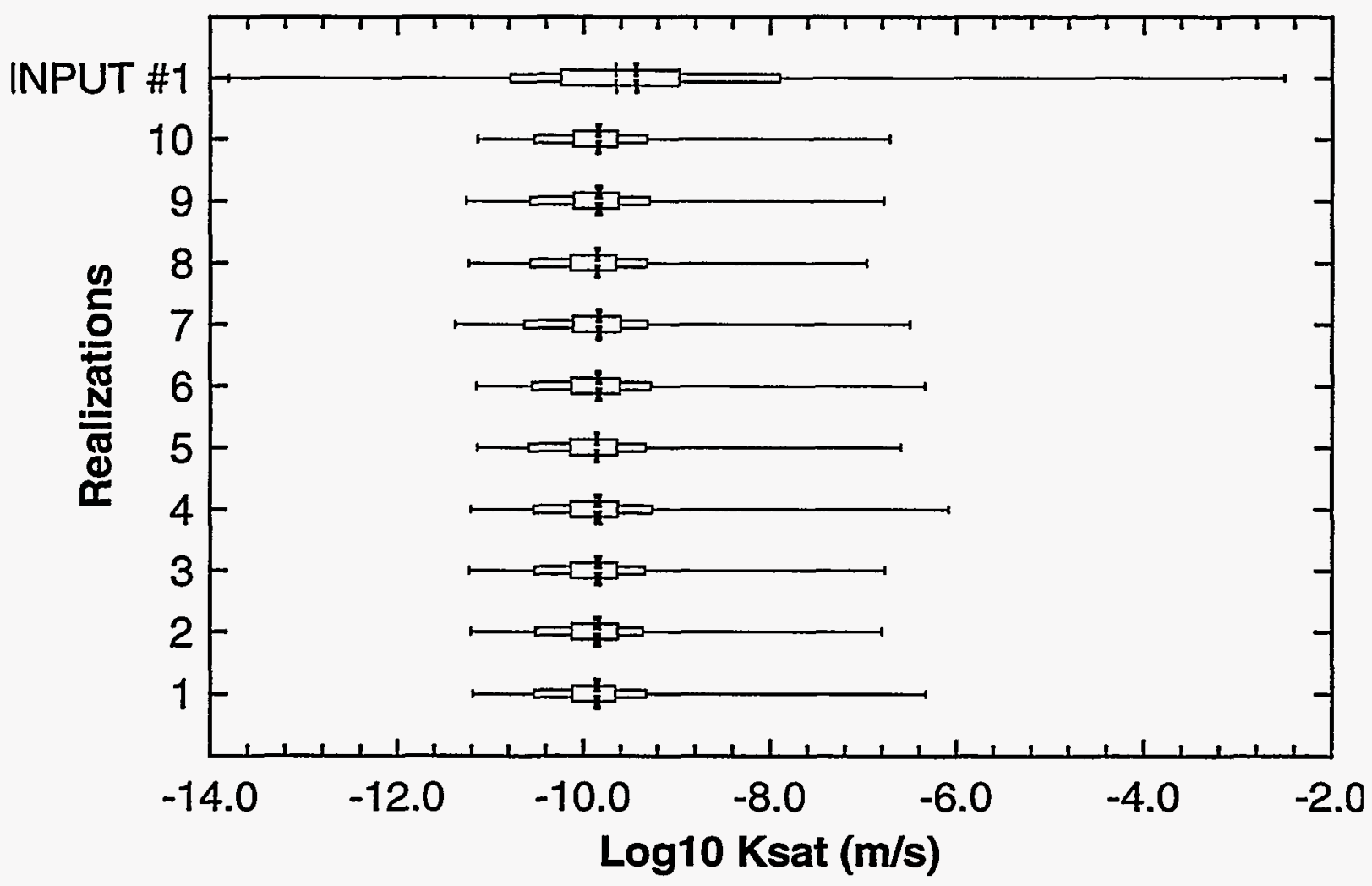

Figure 3-31 Box and whisker plots of the distribution of Ksat for the ten realizations of cross-section AA after upscaling and the geostatistically simulated values for realization number one (INPUT \#1). The thick and thin dashed lines denote the mean and median respectively. The boxes define the 10th, 25th, 75th and 90 th percentiles and the ends of the whiskers are the minimum and maximum values.

\subsection{Summary}

\subsubsection{Evaluation of Rock Properties Models}

The geostatistical simulations are evaluated in a number of ways. The conceptual model of porosity being controlled by the degree of welding is checked against borehole data. The general trend of porosity in the vertical direction has been documented from samples obtained in a number of boreholes. The porosity profile in borehole SD-9 (Figure 3-33(a)) are representative of those across the site. The highest porosity values occur in the PTn2 hydrogeological unit near the top of the stratigraphic section. The lowest porosities occur in the vitrophyre units at the top and bottom of the Topopah Spring welded unit. There is a slight decrease in porosity from top to bottom of the Topopah Spring unit through the repeated sequence of nonlithophysal and 
This page intentionally left blank. 


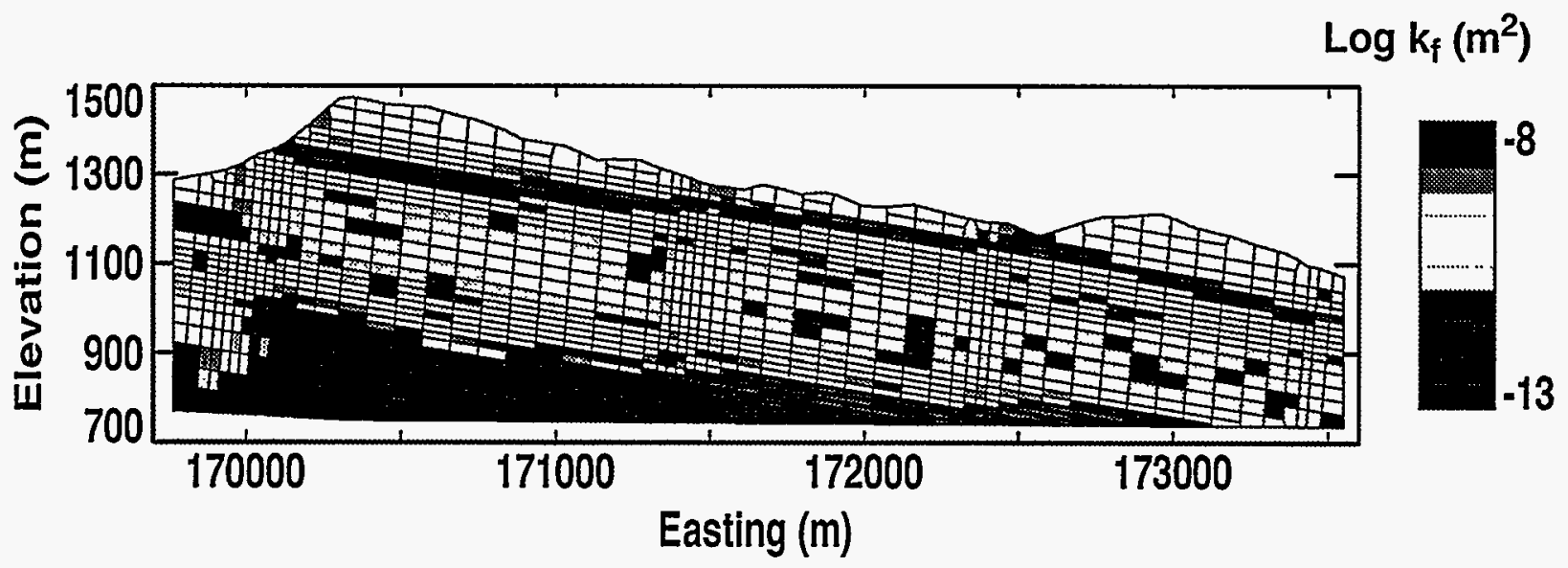

Figure 3-32 Fracture permeability shown in the computational grid for cross-section AA, realization 1 .

Table 3-11: Summary of derivation of parameters at the flow model scale.

\begin{tabular}{|c|c|l|}
\hline Derived Parameter & Derived From & \multicolumn{1}{|c|}{ Method } \\
\hline \hline matrix $\alpha$ & $\begin{array}{c}\text { matrix saturated } \\
\text { hydraulic } \\
\text { conductivity }\end{array}$ & regression (see Figure 3-10) \\
\hline fracture porosity & matrix porosity & $\begin{array}{l}\text { regression (see Figure 3-14) } \\
\text { note that due to the different distributions of } \\
\text { hydrogeological units in the different cross- } \\
\text { sections, multipliers of the regression relationship } \\
\text { were used to maintain a reasonable fracture } \\
\text { aperture distribution (see Section 3.2.2.2) }\end{array}$ \\
\hline fracture aperture & $\begin{array}{c}\text { fracture porosity } \\
\text { and fracture } \\
\text { frequency }\end{array}$ & $\begin{array}{l}\text { direct relationship (see equation 3-2) } \\
\text { note that the fracture frequencies used in these } \\
\text { calculation are those simulated without the } \\
\text { tectonic fracture zones }\end{array}$ \\
\hline $\begin{array}{c}\text { fracture } \\
\text { permeability }\end{array}$ & $\begin{array}{c}\text { fracture frequency } \\
\text { and aperture }\end{array}$ & direct relationship (see equation 3-3) \\
\hline fracture $\alpha$ & fracture aperture & direct relationship (see equation 3-6) \\
\hline
\end{tabular}


lithophysal hydrogeological units (Figure 3-33(a)). Porosity as a function of elevation is extracted from four vertical transects along cross-section AA (Figure 3-21). The trends in these transects (Figure 3-33, (b) through (e)) are compared to the data from borehole SD-9 (Figure 3-33 (a)).

The high porosity of the PTn2 unit and the low porosity of the vitrophyre are reproduced in most profiles. The trend of slightly decreasing porosity values from top to bottom of the Topopah Spring unit is seen in SD-9 (Figure 3-33 (a)) and is well defined in profiles $\mathrm{c}$ and d. This trend is less defined in profiles $b$ and $e$. The generally random appearance of profile $b$ in Figure 333 is due to its location within the Solitario Canyon Fault Zone. The high variability of porosity along profile $b$ is caused by the transect sampling porosity values from unfaulted rock on both sides of the fault zone.

To determine whether or not the GLINTMOD software is able to reproduce the measured mean and variability of porosity within the hydrogeological units, 200 data points were extracted from each hydrogeological unit from the realization of porosity shown in Figure 3-21. The means and standard deviations of the extracted data are shown in Table 3-12. Due to the limited extent of the Prow Pass/Bullfrog welded hydrogeological unit, it was not possible to extract a representative porosity sample from this unit. The means and standard deviations (with the exceptions of units 1 to 3 ) of the simulated hydrogeological units (Table 3-12) compare favorably with the observed statistics for each hydrogeological unit shown in Table 3-1.

Table 3-12: Porosity means and standard deviations for the hydrogeological units as extracted from porosity realization number one of cross-section AA. It was not possible to extract a representative sample from unit \#5 due to its limited extent in cross-section AA. For comparison, the measured porosity means and standard deviations shown in Table 3-1 are repeated here.

\begin{tabular}{|l|c|c|c|c|}
\hline \multirow{2}{*}{$\begin{array}{c}\text { Hydrogeological Unit } \\
\text { Name and Number }\end{array}$} & \multicolumn{2}{|c|}{ Modeled Parameters } & \multicolumn{2}{c|}{ Measured Parameters } \\
\cline { 2 - 5 } & $\begin{array}{c}\text { Mean } \\
\text { Porosity }\end{array}$ & $\begin{array}{c}\text { Porosity } \\
\text { Standard } \\
\text { Deviation }\end{array}$ & $\begin{array}{c}\text { Mean } \\
\text { Porosity }\end{array}$ & $\begin{array}{c}\text { Porosity } \\
\text { Standard } \\
\text { Deviation }\end{array}$ \\
\hline \hline Vitrophyre (1) & 0.06 & 0.01 & 0.05 & 0.05 \\
\hline
\end{tabular}



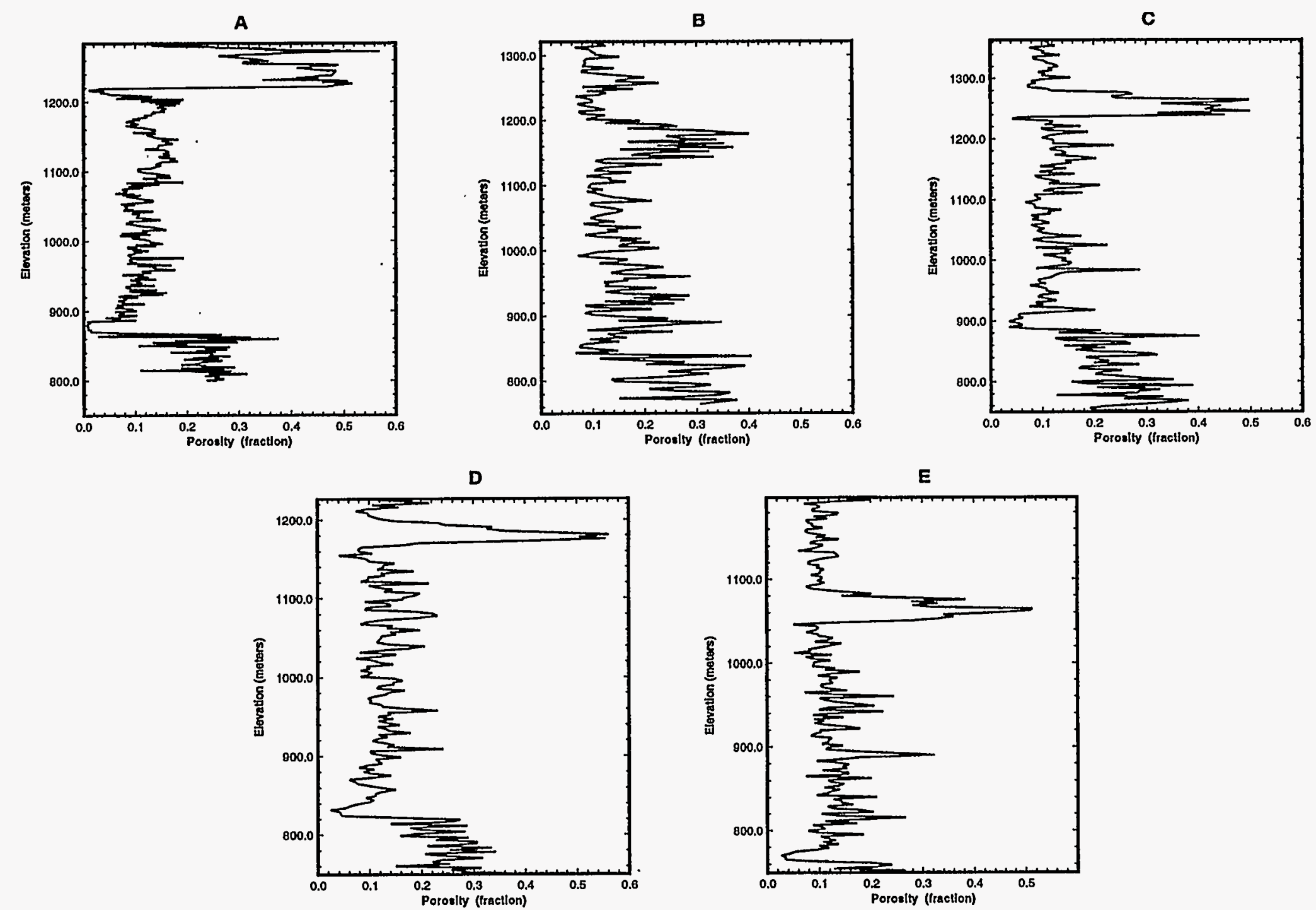

Figure 3-33 Comparison of porosity trends in boring SD-9 (A) with four vertical profiles taken along cross-section AA from porosity realization number one. The profiles were extracted at the easting coordinates: $170,000 \mathrm{~m}(\mathrm{~B})$, $171,000 \mathrm{~m}(\mathrm{C}), 172,000 \mathrm{~m}(\mathrm{D})$ and $173,000 \mathrm{~m}(\mathrm{E})$. 
Table 3-12: Porosity means and standard deviations for the hydrogeological units as extracted from porosity realization number one of cross-section AA. It was not possible to extract a representative sample from unit \#5 due to its limited extent in cross-section AA. For comparison, the measured porosity means and standard deviations shown in Table 3-1 are repeated here.

\begin{tabular}{|c|c|c|c|c|}
\hline \multirow[b]{2}{*}{$\begin{array}{l}\text { Hydrogeological Unit } \\
\text { Name and Number }\end{array}$} & \multicolumn{2}{|c|}{ Modeled Parameters } & \multicolumn{2}{|c|}{ Measured Parameters } \\
\hline & $\begin{array}{l}\text { Mean } \\
\text { Porosity }\end{array}$ & $\begin{array}{c}\text { Porosity } \\
\text { Standard } \\
\text { Deviation }\end{array}$ & $\begin{array}{l}\text { Mean } \\
\text { Porosity }\end{array}$ & $\begin{array}{c}\text { Porosity } \\
\text { Standard } \\
\text { Deviation }\end{array}$ \\
\hline Tiva Cliff welded (2) & 0.10 & 0.02 & 0.09 & 0.07 \\
\hline TSw non-lithophysal (3) & 0.11 & 0.02 & 0.10 & 0.04 \\
\hline TSw lithophysal (4) & 0.14 & 0.04 & 0.13 & 0.04 \\
\hline PP-BF welded (5) & NA & NA & 0.15 & 0.04 \\
\hline Calico Hills (6) & 0.26 & 0.07 & 0.23 & 0.07 \\
\hline PP-BF non-welded (7) & 0.26 & 0.08 & 0.25 & 0.06 \\
\hline $\operatorname{PTn} \# 2$ (8) & 0.29 & 0.07 & 0.28 & 0.10 \\
\hline PTn \#1 (9) & 0.41 & 0.08 & 0.46 & 0.08 \\
\hline
\end{tabular}

The relationship between porosity and Ksat modeled through both linear coregionalization and a linear regression model is checked for both the zeolitized and non-zeolitized portions of the cross-section. Figure 3-34 shows the regression relationships for the simulated properties. The regression relationships honor the equations derived from the sample data. The simulated results for the zeolitized portion of the cross-section have a higher coefficient of determination than the measured data (Figure 3-6). This higher degree of correlation in the simulated results relative to the measured data, is attributed to a lack of outlier points in the simulated values and an imposed gaussian distribution of errors about the regression line compared to non-gaussian errors in the field data. The regression relation from the simulated values also extends to higher porosity values than the regression from the field data. This extension is an artifact of the modeling process, in that porosity values are simulated independently of the Ksat values. The field data reflect an upper limit for porosity in the neighborhood of 35 percent for the zeolitized units (Figure 3-6). As seen in Figure 3-33b, the majority of the porosity values are below 35 percent. However, porosity values above 35 percent are simulated in the zeolitized zone and then the regression model is applied to them to determine a value of Ksat. The use of a cosimulation algorithm instead of linear coregionalization would correct this problem and keep the simulated results closer to the measured results.

The conceptual models of fracturing and faulting are evaluated by visual inspection of the resulting simulations. Examination of Figure 3-27 shows the deterministically modeled faults to be in the correct locations with the correct dips and widths. The randomly simulated high fracture 
(a)

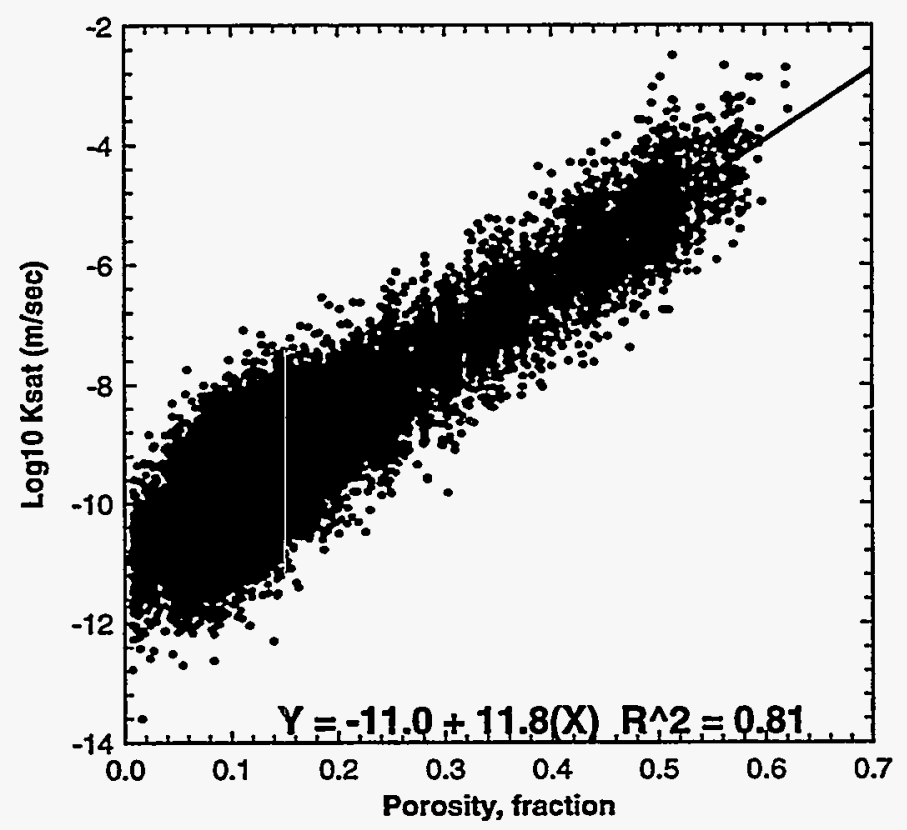

(b)

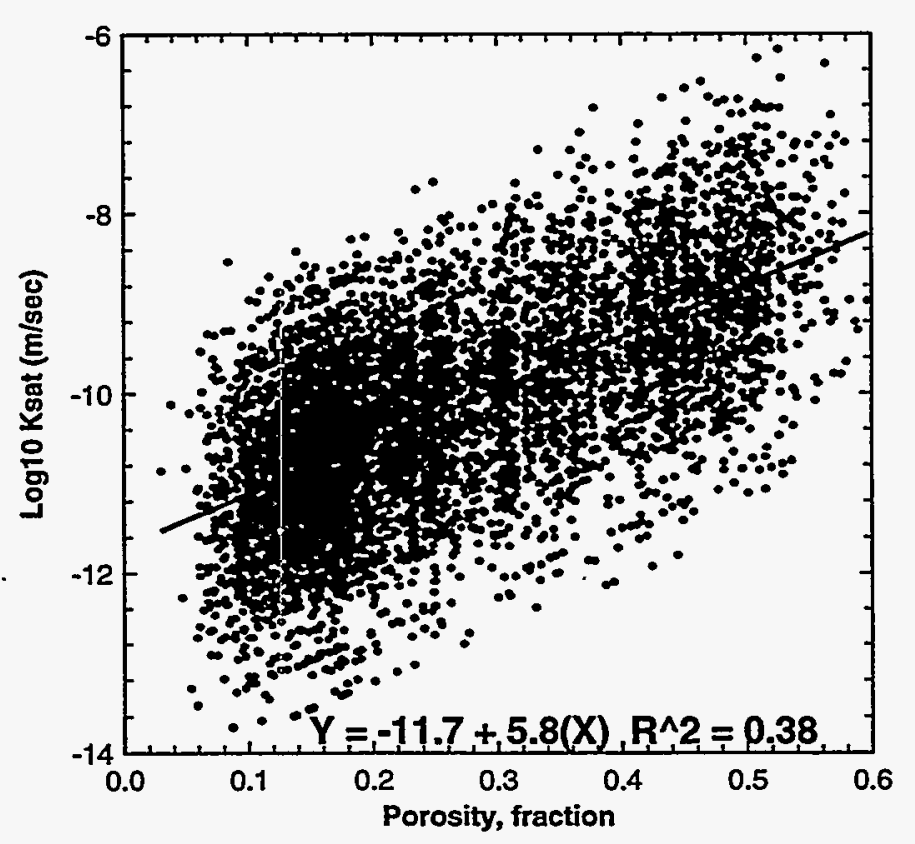

Figure 3-34 Regression relationship between simulated matrix porosity and Ksat for the non-zeolitized (a) and zeolitized (b) portions of the mountain resulting from the linear coregionalization and the regression models applied to the matrix porosity realization shown in Figure 3-6. 
frequency zones are of the prescribed length, orientation and frequency and the fracture frequency within the zones is sharply reduced across the PTn2 and Calico Hills hydrogeological units (Figure 3-27).

The end result of the geostatistical simulation process is to transfer the uncertainty in the spatial distribution of rock properties that results from limited sampling through a transfer function (here, an unsaturated flow model), to a distribution of travel times. The modeled level of uncertainty is evaluated qualitatively by noting that an expectation map of any simulated property should approach the image of the deterministic geologic model (Figure 3-3(a) for cross-section $\mathrm{AA})$ as the number of realizations used to create the expectation map increases. A porosity expectation map created by averaging the porosity values from 10 realizations on a element by element basis is shown in Figure 3-35. This map exhibits strong similarity to the deterministic conceptual model in Figure 3-3 (a) in terms of the location and geometry of the hydrogeological units.

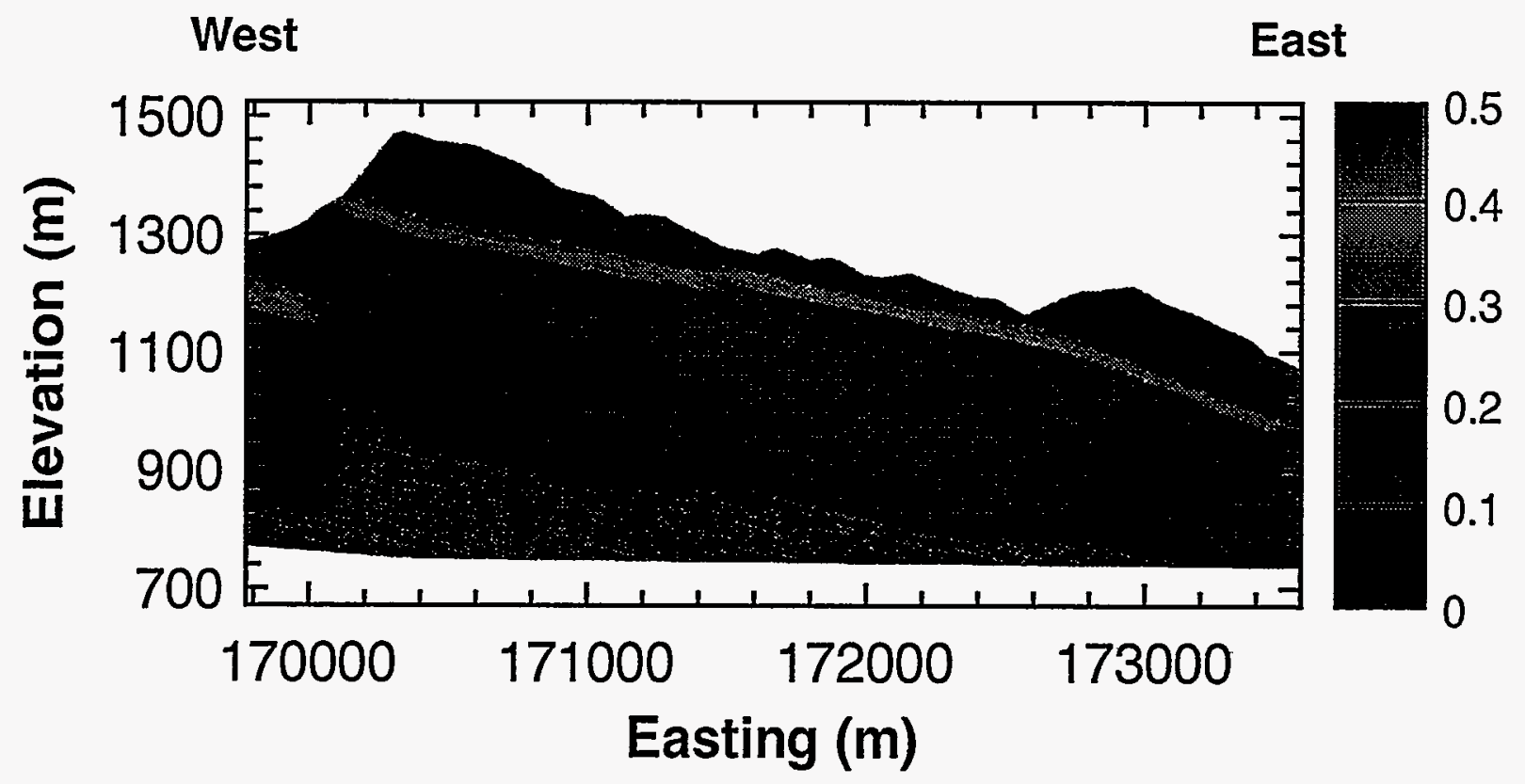

Figure 3-35 Expectation map of matrix porosity created by averaging the porosity on a pixel by pixel basis across 10 realizations created for cross-section AA. Compare the location and geometry of the regions of similar porosity to the locations and geometry of the hydrogeological units in Figure 3-3 (a).

When all of the simulated rock properties are upscaled and implemented into the flow model TOUGH2 (Pruess, 1991) along with measured and inferred boundary conditions, resulting matrix saturations can be compared with measured saturations and the variability in modeled saturations between realizations can be assessed. This assessment is accomplished and discussed in Chapter 4. 


\subsubsection{Discussion}

In addition to conditioning rock property data, conceptual models of the geology and stratigraphy of a site can be used to guide the geostatistical simulation process. In this situation, the geostatistician must act as the intermediary between the field geologist and the groundwater flow modeler. The conceptual models developed in the field must be retained through the modeling and upscaling process as they are turned into numerical representations of site conditions.

Results herein indicate that conceptual models derived from site investigations and geologic interpretation can be readily incorporated into a stochastic modeling exercise. By coupling the simulation of porosity with a geologic framework model, the resulting realizations reflect the stratigraphic control of porosity and, on average, reproduce the geologic framework model. Combining gaussian simulation with linear coregionalization has reproduced the measured relationship between porosity and Ksat across the model domain. A further combination of gaussian simulation, conditioned to the geologic framework model, and indicator simulation produced stochastic realizations of stratigraphically controlled fracture frequency overprinted by high fracture frequency zones resulting from tectonic activity.

In a stochastic modeling exercise, it is the random component of the process that gives the results their variability. The true amount of spatial variability is never known, but can only be estimated. Evaluation of whether or not the actual variability is being estimated accurately can be made by comparing measured and modeled results. In this study, the vertical profiles extracted from a simulation of porosity indicate that the modeling process is correctly representing the vertical trends of the measured porosity. Additionally, the global mean and standard deviation of porosity within hydrogeological units matches those parameters as measured in the field. 
This page intentionally left blank. 


\section{CHAPTER 4 \\ Numerical Flow Modeling in the Unsaturated Zone}

The conceptual model of groundwater flow in the unsaturated zone, as described in Section 2.4 of this report, is implemented for analysis of groundwater travel time using numerical methods. This chapter contains a description of the implementation of the relevant boundary conditions and governing equations of unsaturated flow. The simulated results of these flow calculations are presented and compared to borehole observations. The particle tracking method used to determine the distribution of travel times from the flow simulations is described and the results are documented. Finally, sensitivity analyses are presented in an attempt to delineate important uncertainties in the analysis and to illustrate potentially significant processes.

\subsection{Boundary Conditions}

The four two-dimensional cross-sections introduced in the previous chapter maintain similar boundary conditions. Laterally, the domains are bounded by no-flow conditions. On the bottom of each domain, the grid is bounded by a row of saturated elements (i.e. the water table). Finally, the top row of elements of each cross-section is specified with a spatially non-uniform infiltration (generation) rate (in the dual permeability model (DKM) simulations, the infiltration is applied only to the fracture elements). The infiltration rates used in this study are based on distributions calculated by Hudson and Flint ${ }^{22}$ for the Yucca Mountain site. Note that the infiltration rates used in these analyses were taken from Hudson and Flint ${ }^{23}$ while the report was still in review. Therefore, values in the published version of Hudson and Flint ${ }^{24}$ may differ somewhat from those used in these analyses. Their average annual infiltration rate is nearly two orders of magnitude larger than previous estimates ( $>20 \mathrm{~mm} / \mathrm{year}$ ), and the authors caution that the absolute values of these estimates are preliminary. They mention that the methods used were appropriate for estimating shallow infiltration, but potential processes (such as evaporation below depths of $2 \mathrm{~m}$ ) affecting the net infiltration may not have been included. As a result, their infiltration distribution is reduced by two orders of magnitude as a base case in this study, but their spatial distribution of relative infiltration is retained. The decision to reduce the infiltration rate by this amount is based on sensitivity studies (Section 4.5.1). Figures 4-1 a to d show each crosssection and the corresponding infiltration distribution that was applied to the top row of elements. The units are presented in $\mathrm{mm} / \mathrm{year}$, but the infiltration rates were converted to $\mathrm{kg} / \mathrm{sec}$ for use in the numerical simulations using the density of water at $20^{\circ} \mathrm{C}$ and the horizontal cross-sectional area of each element in the top row.

22. see footnote 2 , page 9 .

23. Ibid.

24. Ibid. 


\subsection{Numerical Simulations of Flow}

In previous sections, the development of necessary hydrologic model parameters and boundary conditions has been described so that numerical simulations of flow through the UZ can be performed. Each of the four two-dimensional cross-sections of interest has been discretized, and properties have been simulated and upscaled so that each element in the computational grid contains its own unique set of hydrologic properties and characteristic curves. This section presents the governing equations that are implemented in the numerical simulations, as well as other relevant issues pertaining to the modeling efforts.

\subsubsection{Governing Equations}

The system under consideration consists of isothermal, unsaturated groundwater flow in a heterogeneous, fractured domain. The gas phase is assumed to be passive with a constant pressure throughout the domain so that only the liquid-phase is considered in the flow equations ${ }^{25}$. The governing equations for this system follow from conservation of mass:

$$
\frac{\partial}{\partial t}(\rho S \phi)+\nabla \bullet q=0
$$

and Darcy's law:

$$
q=-\frac{k k_{r}}{\mu} \rho\left(\nabla P_{c}-\rho g\right)
$$

where $q$ is the Darcy flux, $\rho$ is the liquid density, $S$ is the liquid saturation, $\phi$ is the porosity, $k$ is the permeability, $k_{r}$ is the relative permeability, $\mu$ is the liquid viscosity, $P_{c}$ is the capillary pressure, and $g$ is the gravitational acceleration. Combination of equations (4-1) and (42) yields the transient form of Richards equation (Freeze and Cherry, 1979). Equation (4-1) is a highly non-linear partial differential equation because of the dependence of the relative permeability and saturation on capillary pressure. Solution of equation (4-1) in a completely heterogeneous fractured domain can be numerically difficult, but prior analyses have demonstrated the implementation of this formulation for problems similar to this study (Ho et al., 1995).

Because the computational domains are comprised of both fracture and matrix materials, the implementation of parameters in equations (4-1) and (4-2) depends on the conceptual model of flow through the fractured rock. In the equivalent continuum model (ECM) (refer to Section 2.3), the material parameters in equations (4-1) and (4-2) are comprised of both fracture and matrix properties for a given computational element assuming pressure equilibrium between the fracture and matrix:

25. Sensitivity analyses comparing two-phase and single-phase numerical simulations of infiltration systems similar to those presented in this study showed little differences in the resulting saturation and flux profiles (Ho et al., 1995). However, computational times were significantly reduced by using the singlephase model. 
West

East
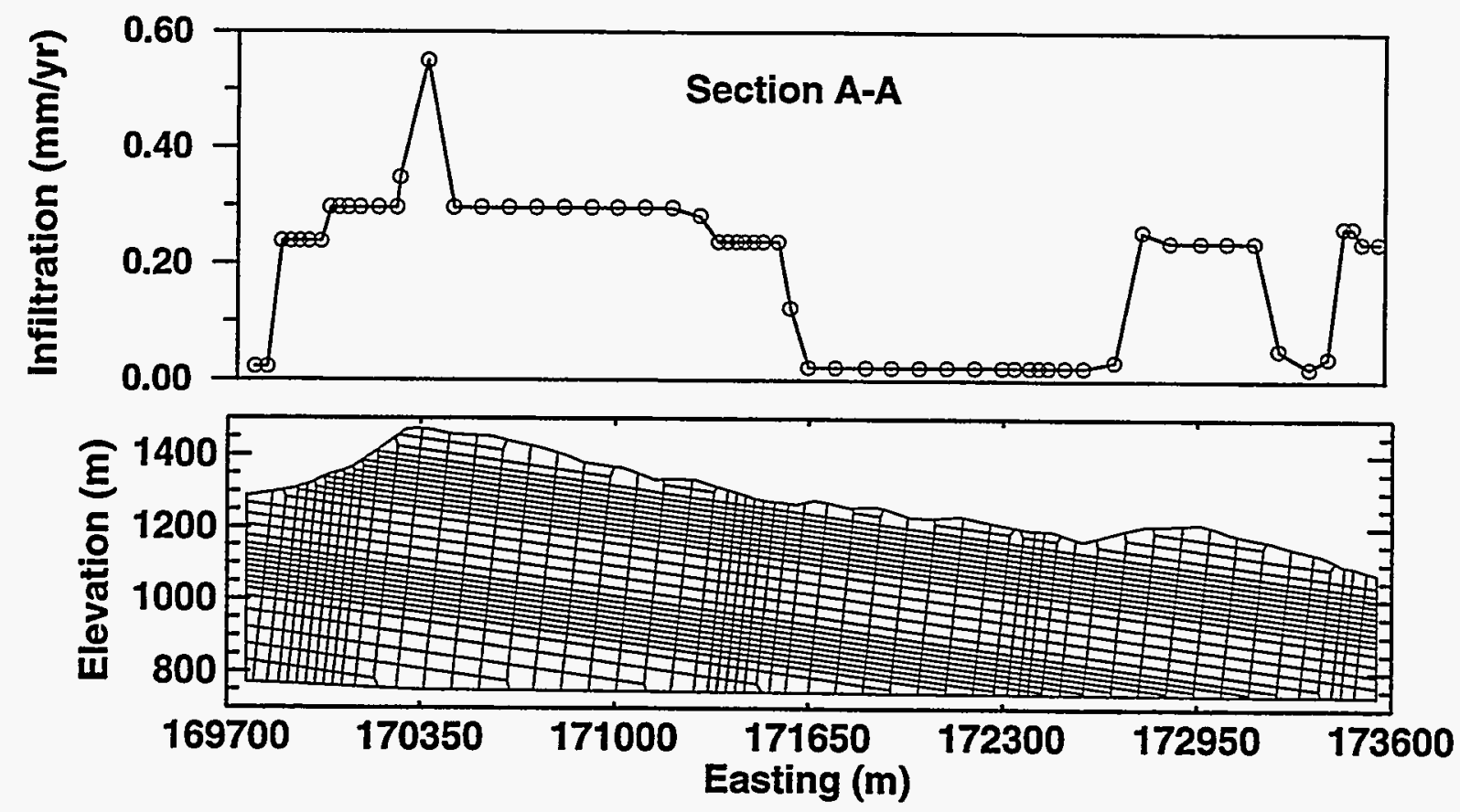

(a)

West

East
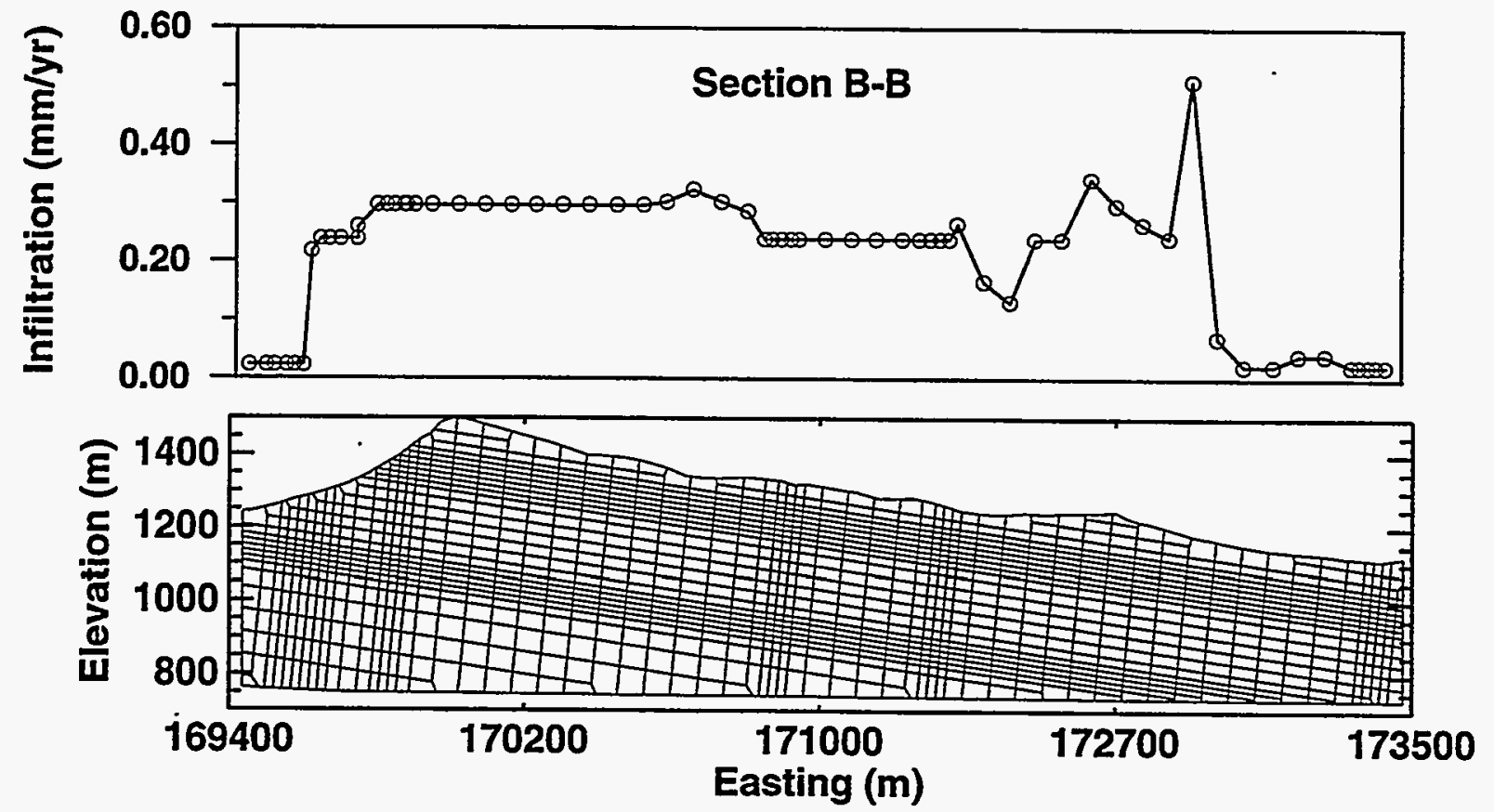

(b)

Figure 4-1 $a$ and $b$ Infiltration rates and corresponding numerical grids for a) crosssection A-A and b) cross-section B-B. 


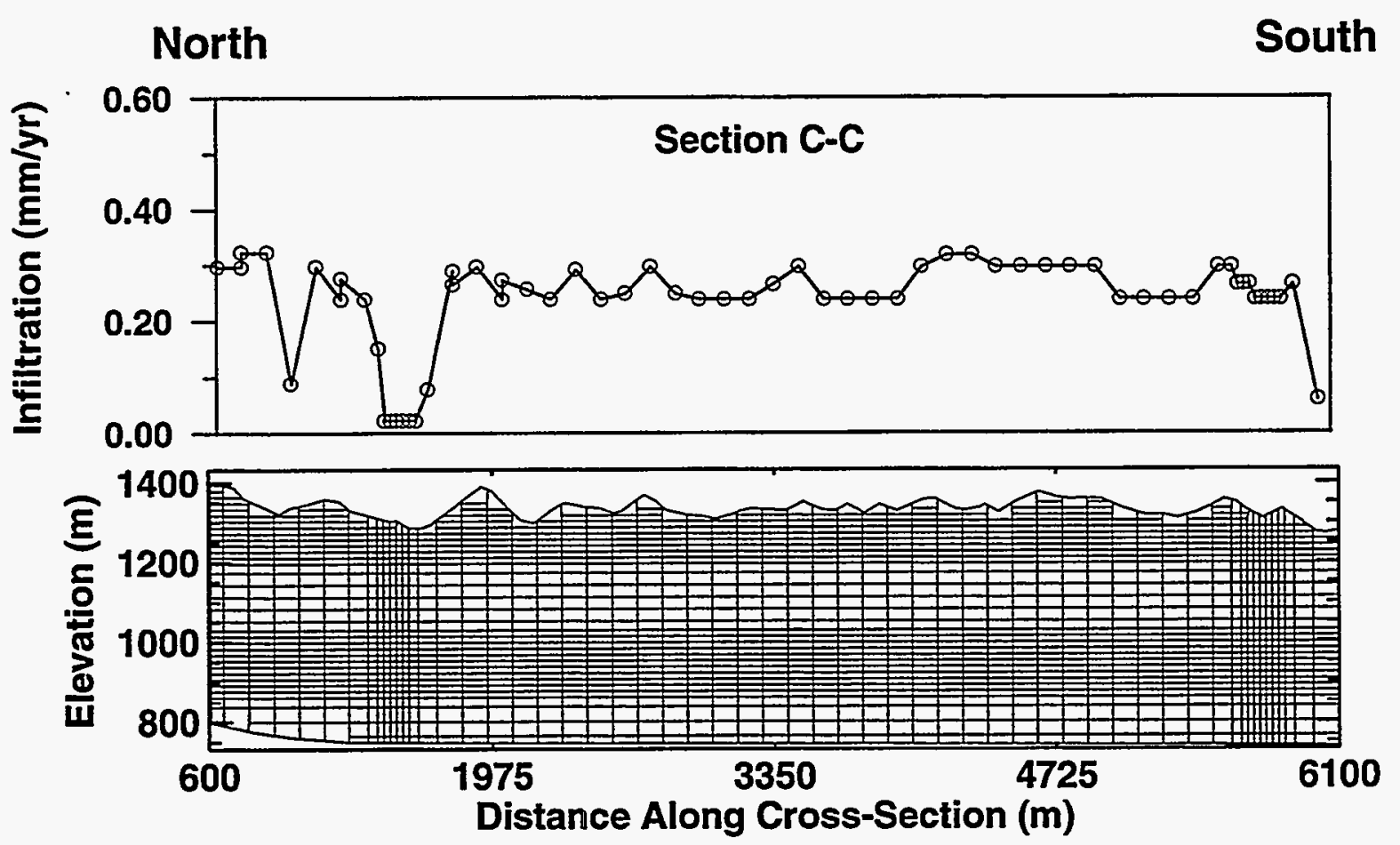

(c)

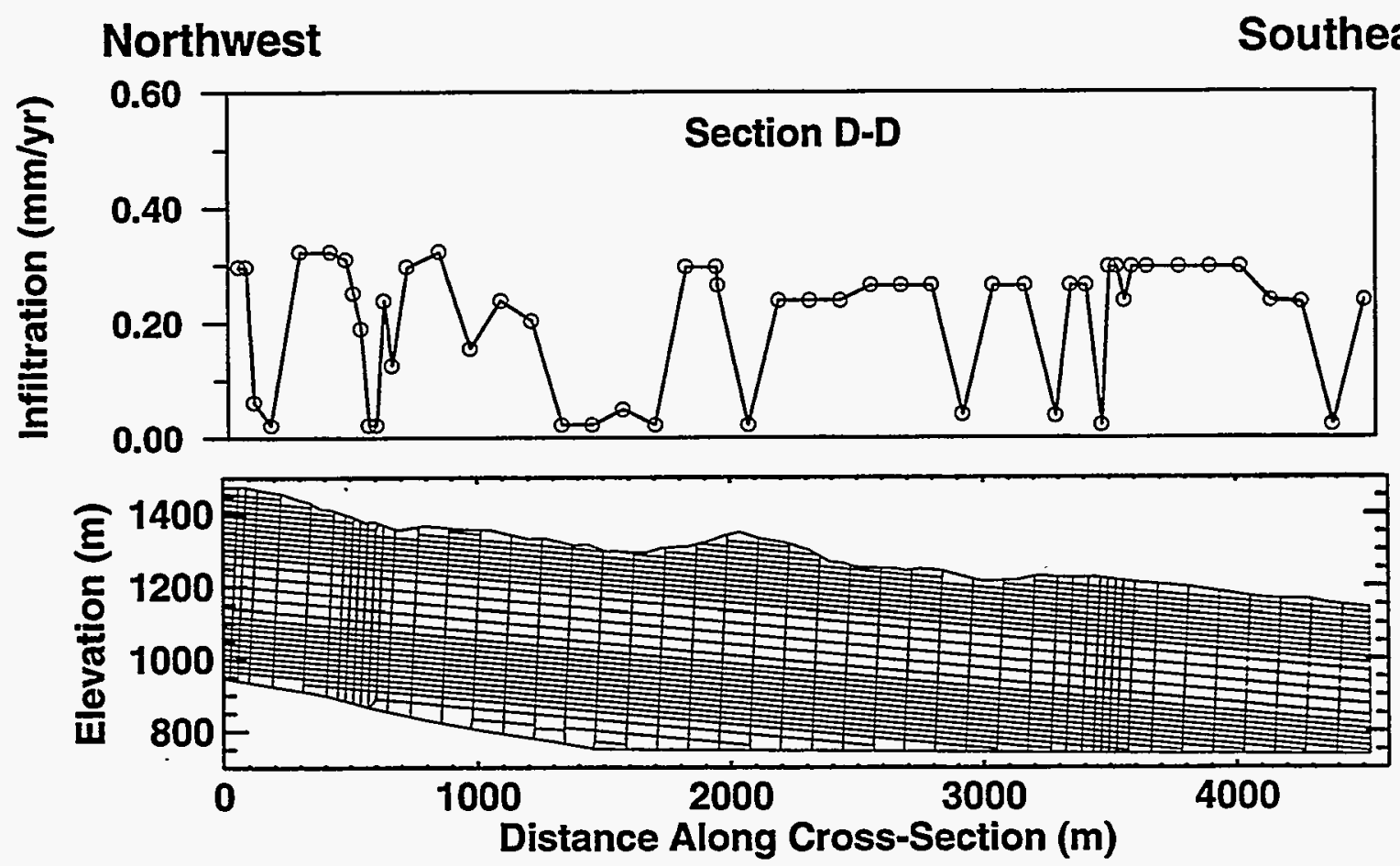

(d)

Figure 4-1 $\mathrm{c}$ and $\mathrm{d}$ Infiltration rates and corresponding numerical grids for $\mathrm{c}$ ) crosssection C-C and d) cross-section D-D. 


$$
\begin{gathered}
\phi=\phi_{f}+\left(1-\phi_{f}\right) \phi_{m} \\
S=\frac{S_{f} \phi_{f}+S_{m}\left(1-\phi_{f}\right) \phi_{m}}{\phi_{f}+\left(1-\phi_{f}\right) \phi_{m}} \\
k=k_{m}\left(1-\phi_{f}\right)+k_{f} \phi_{f}
\end{gathered}
$$

where subscript $m$ and $f$ denote the matrix and fracture materials, respectively. Since unsaturated flow is being considered, equations (4-3) - (4-5) must be used in conjunction with expressions relating liquid saturations and relative permeabilities to capillary pressure. The van Genuchten characteristic functions are used for this purpose:

$$
\begin{gathered}
S_{j}=S_{r, j}+\left(1-S_{r, j}\right)\left(1+\left|\alpha_{j} P_{c}\right|^{\beta_{j}}\right)^{-\lambda} \\
k_{r, j}=\frac{\left(1-\left|\alpha_{j} P_{c}\right|^{\beta_{j}-1}\left(1+\left|\alpha_{j} P_{c}\right|^{\beta_{j}}\right)^{-\lambda_{j}}\right)^{2}}{\left(1+\left|\alpha_{j} P_{c}\right|^{\beta_{j}}\right)^{\lambda_{j} / 2}}
\end{gathered}
$$

where

$$
\lambda_{j}=1-\frac{1}{\beta_{j}}
$$

and

$$
j=\text { matrix or fracture }
$$

Equations (4-6) and (4-7) are applicable for both the matrix and fracture materials (as denoted by the subscript $j$ ) where $\alpha$ and $\beta$ are van Genuchten characteristic parameters. In the ECM, the same capillary pressure exists in both the fracture and matrix materials in a given computational element. For a given $P_{c}$ (from equation (4-2)), equation (4-6) is used to determine the matrix and fracture saturations for the ECM. Equation (4-4) is then used to obtain an effective saturation for equation (4-1). Equation (4-7) is used to obtain the matrix and fracture relative permeabilities corresponding to the given $P_{c}$, which are combined to yield an effective relative permeability for use in equation (4-2):

$$
k_{r}=\frac{k_{r, m} k_{m}\left(1-\phi_{f}\right)+k_{r, f} k_{f} \phi_{f}}{k}
$$

Equations (4-3) to (4-8) describe how the material parameters are combined in the ECM for implementation into the governing equations (4-1) and (4-2). The end result is a computational domain that behaves mathematically as if it were a single porous medium. Note, however, that equations (4-3) to (4-8) can be used to back-calculate the saturations and fluxes associated with the matrix and fractures once the effective saturations and fluxes are determined from equations (4-1) and (4-2).

Groundwater flow pore velocity is calculated from the Darcy velocity as follows: 


$$
v_{j}=\frac{q_{j}}{\theta_{j}-\theta_{\text {resid }}}
$$

where:

$$
\theta_{j}=S_{j} \phi_{j}
$$

and $\theta_{\text {resid }}$ is the residual saturation. Use of equation 4-9 implies that the liquid held as residual saturation is immobile and does not participate in flow.

In the DKM, the fracture and matrix domains are modeled separately. Therefore, the parameters associated with equations (4-1) and (4-2) correspond directly to either a fracture or matrix property, rather than an effective parameter as used in the ECM. The flow between fracture and matrix elements in the DKM is modeled with Darcy's law (equation (4-2)) as shown in Figure 2-3, where the matrix properties are used for the mobility terms. The connection area between the fracture and matrix elements is calculated based on the geometry of a uni-directional set of fractures embedded in the matrix. The geometric connection area between a fracture and matrix element is calculated as twice the grid volume times the fracture frequency within that grid (Ho et al., 1995 (Appendix B)). However, because of small-scale processes discussed in section 2.3 , the geometric connection area between the fractures and matrix is reduced by three orders of magnitude as a base-case in this study. In addition, the calculated distance between adjoining fracture and matrix elements is based on methods described by Pruess (1983) and Warren and Root (1963). Since only one element is used to describe the matrix, the distance, $d$, used in the equation for flow between fractures and matrix (Figure 2-3) is determined such that the quasisteady flux is comparable to the flux between a fracture and a continuous matrix unit. The distance, $d$, is calculated as the fracture spacing divided by six (Ho et al., 1995 (Appendix B)) for each grid.

Conceptually, the DKM formulation is more straightforward with regards to the implementation of the governing equations. However, this requires that the computational mesh used by the DKM contains two elements (a fracture and a matrix element) for every ECM element, which increases the computational burden of the solution.

\subsubsection{Numerical Grid}

A numerical grid is created for each of the four cross-sections described in Chapter 3 (Figure 4-1a to 4-1d). The grids are refined in areas of "interesting" geologic features such as the PTn, basal vitrophyre, and faults. The size of each element in the unrefined areas is roughly 50 meters high by 100 meters wide. The number of elements in each grid is summarized in Table 4-1.

The DKM contains twice as many elements as the corresponding ECM, but the refinement remains the same. To understand this concept, consider two identical meshes overlaid on top of each other. One of the meshes represents the matrix continuum, while the superposed mesh 
Table 4-1: Number of elements in each computational grid.

\begin{tabular}{ccccc}
\hline & \multicolumn{4}{c}{ Cross-Section } \\
& A-A & B-B & C-C & D-D \\
\hline ECM & 1441 & 1350 & 1700 & 1342 \\
DKM & 2882 & 2700 & 3400 & 2684 \\
\hline
\end{tabular}

represents the fracture continuum. For each element location, the matrix element is connected to its corresponding fracture element. The coupling depends on parameters such as fracture spacing and connection area (described in Section 2.3.2 and Ho et al., 1995 (Appendix B)).

\subsubsection{Numerical Solution Using TOUGH2}

The numerical code TOUGH2 (SNL software quality assurance configuration management version 3.1) is used in this study based on preliminary assessments by $\mathrm{Ho}$ et al. (1995). TOUGH2 (Transport of Unsaturated Groundwater and Heat) simulates transient, multiphase, non-isothermal flow in one, two, or three-dimensions using the integral finite difference method (Pruess, 1987; Pruess, 1991). In the integral finite difference method, no reference is made to a global coordinate system, so volume elements of arbitrary size and shape can be used. TOUGH2 incorporates equation-of-state modules (used interchangeably) that govern more specific features of the system, such as the single-phase flow module used in this study (EOS9).

The governing equations are solved throughout the entire computational domain using an iterative conjugate gradient solver. Since TOUGH2 does not implement a steady-state solution per se, steady-state simulations are achieved by running the code until the total outflow of water at the water table boundary equals the total infiltration as specified along the top row of elements. The initial conditions used for these steady-state runs were set to a constant saturation of 0.5 . Because these initial conditions are drier in most areas than the steady-state solution, the flux at the lower boundary should increase with time, achieving equality with the infiltration flux only when steady-state flow conditions are reached. Generally speaking, steady-state conditions are reached within a simulated time of $10^{8}$ years. The actual duration of these simulations corresponds to a clock time on the order of one hour for the DKM simulations and on the order of several minutes for the ECM simulations using a SUN SPARC10 machine.

Ten realizations are simulated using the DKM for each of the four cross-sections introduced in Chapter 3. Steady-state conditions are achieved in each case, yielding steady fracture and matrix saturations and pore velocities that are used in the calculation of groundwater travel times. In addition, the ECM model is used as a basis for comparison with the DKM in several of the simulations. These and other sensitivity analyses are presented in Section 4.5. 


\subsection{Flow Modeling Results and Discussion}

Several parameters are examined in order to interpret the flow model results: matrix and fracture saturations within the cross-section and flow velocities in the matrix and fractures.

Patterns of these parameters are of interest as well as how much an individual parameter varies from one geostatistical realization to another. In addition, as a method to determine that flow modeling results are reasonable, comparisons are made between measured saturations in drillhole core samples and simulated saturations in vertical columns of elements in the cross-sections where the cross-section is near a borehole.

\subsubsection{Matrix Saturations}

Similar trends in matrix saturations for the 10 realizations of each of the 4 cross-sections are as follows (Figure 4-2a to 4-2d):

1) Saturations in the PTn are generally lower than other units, with values ranging from approximately 0.1 to 0.5 .

2) Saturations are generally lower in areas under the washes, where the infiltration is lower.

3) Areas where the non-welded Calico Hills and Prow Pass units are zeolitized are nearly fully saturated $(>0.9)$.

4) Vitrophyres generally show high saturations relative to other, higher porosity units.

The following interpretations can be made for these observations. The lower saturations in the PTn are commonly observed and can be explained by the larger pore spaces in the nonwelded unit. The higher saturations in the zeolitized areas are not explained by a decreased pore space but instead by decreased hydraulic conductivity, that inhibits the flow of water through these areas. The high matrix saturations in the vitrophyres are explained by the decreased pore space (low porosity).

Matrix saturations of two cross-sections are compared where the two cross-sections intersect (Figure 4-3). There are a total of four intersections, with cross-section C-C intersecting the three other cross-sections and cross-section A-A intersecting D-D (Figure 3-1). This comparison of saturations is made to examine self-consistency within the GWTT-95 procedure and to examine the variation in saturations of the 10 realizations along a one dimensional column. In general, the saturations at the intersection of any two cross-sections match very well. The largest differences exist at the intersection of cross-sections AA and DD at elevations between 900 and $1100 \mathrm{~m}$. There are two possible explanations for the lower saturations simulated in crosssection AA. First, examination of Figure 4-2a shows that the PTn is two elements thick along the column, where as for cross-section DD (Figure 4-2d), the PTn in only one element thick. The differences must be due to the different resolutions of the grids for the two cross-sections. It is, therefore, possible that more water is imbibed into the matrix in the PTn in cross-section AA (due to the layer being thicker), leading to lower saturations in the underlying units. Examination of 

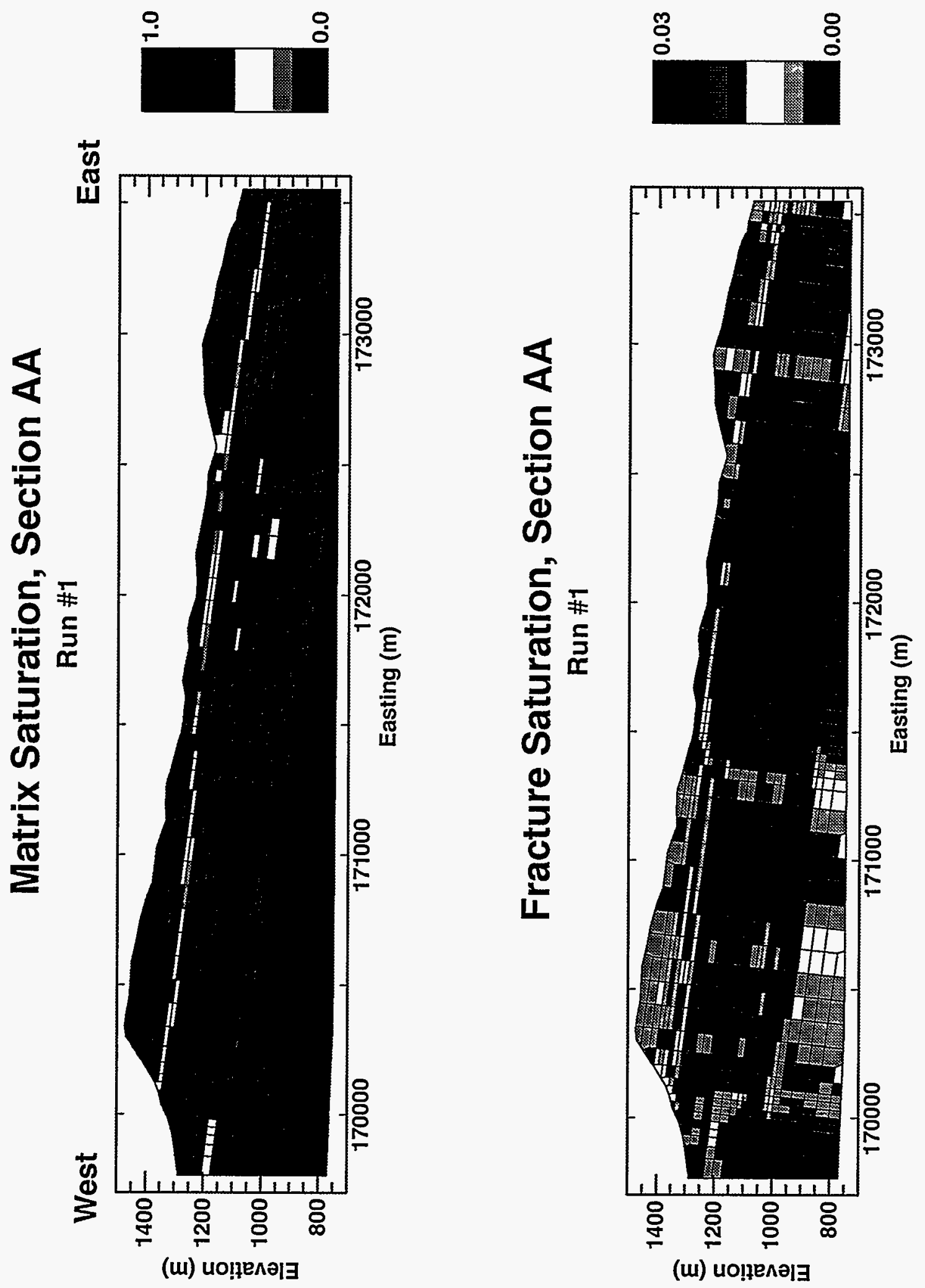

Figure 4-2a Matrix and fracture saturations for cross-section AA, realization 1, dual permeability model. 

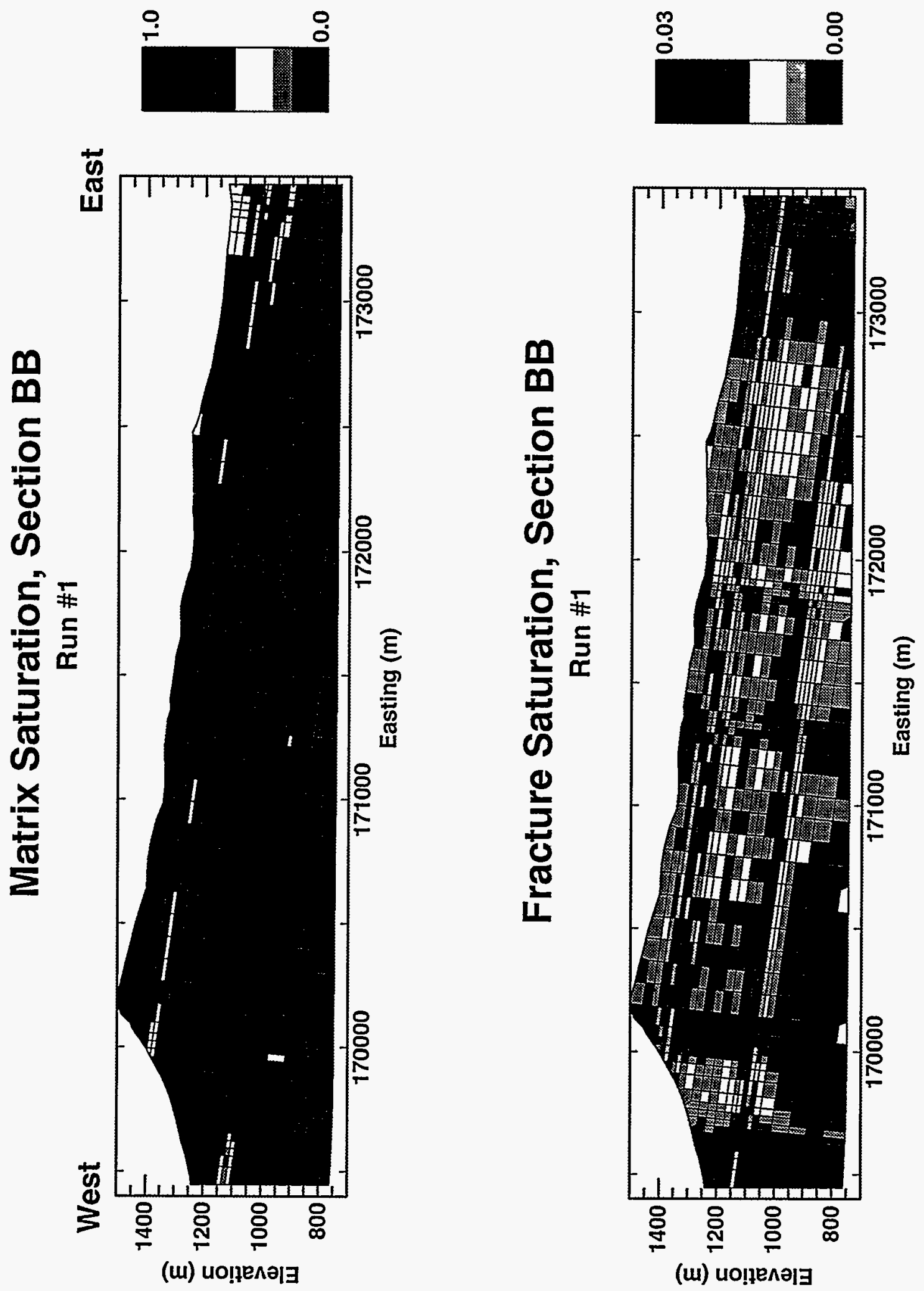

Figure 4-2b Matrix and fracture saturations for cross-section BB, realization 1, dual permeability model. 


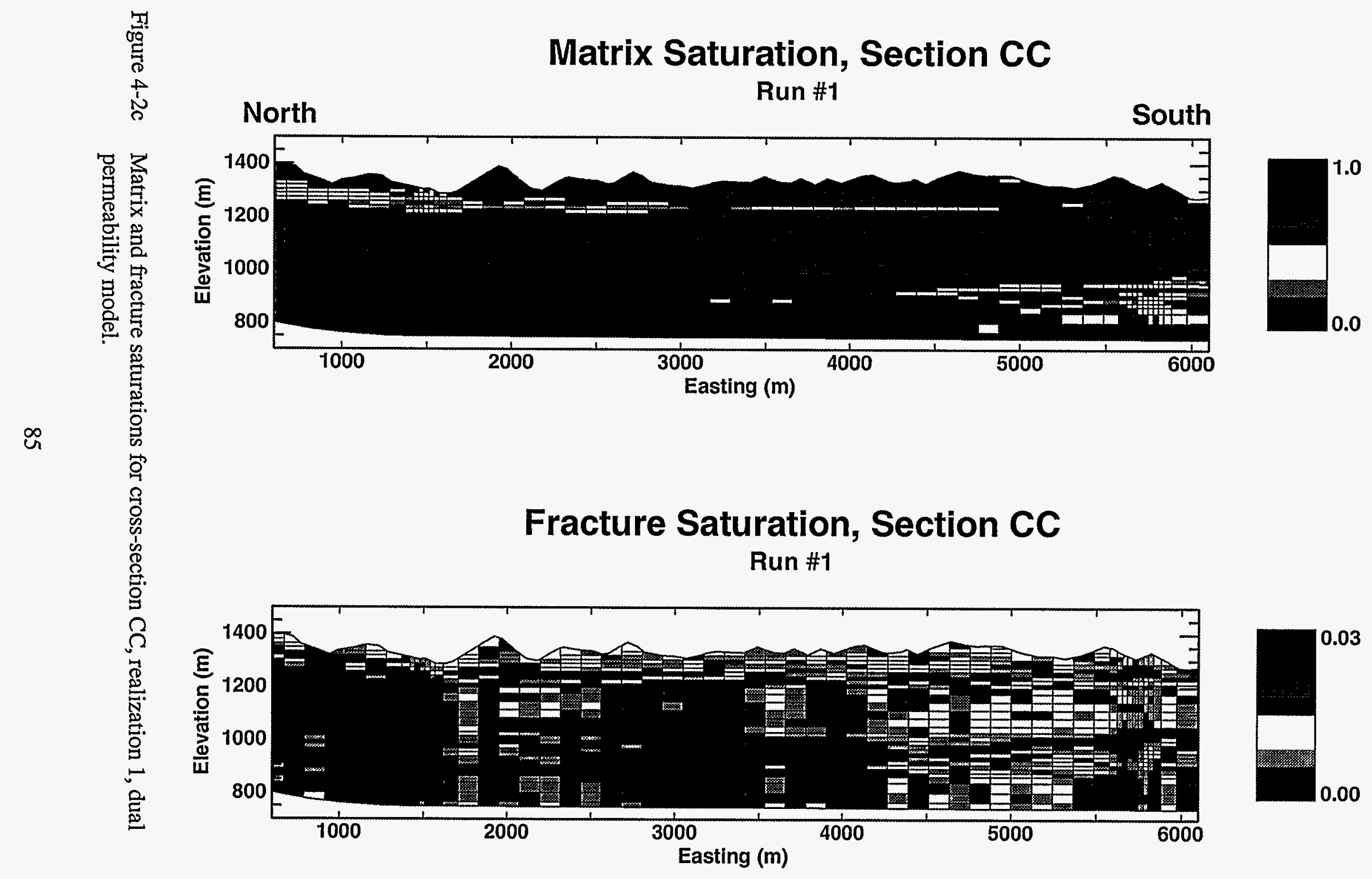




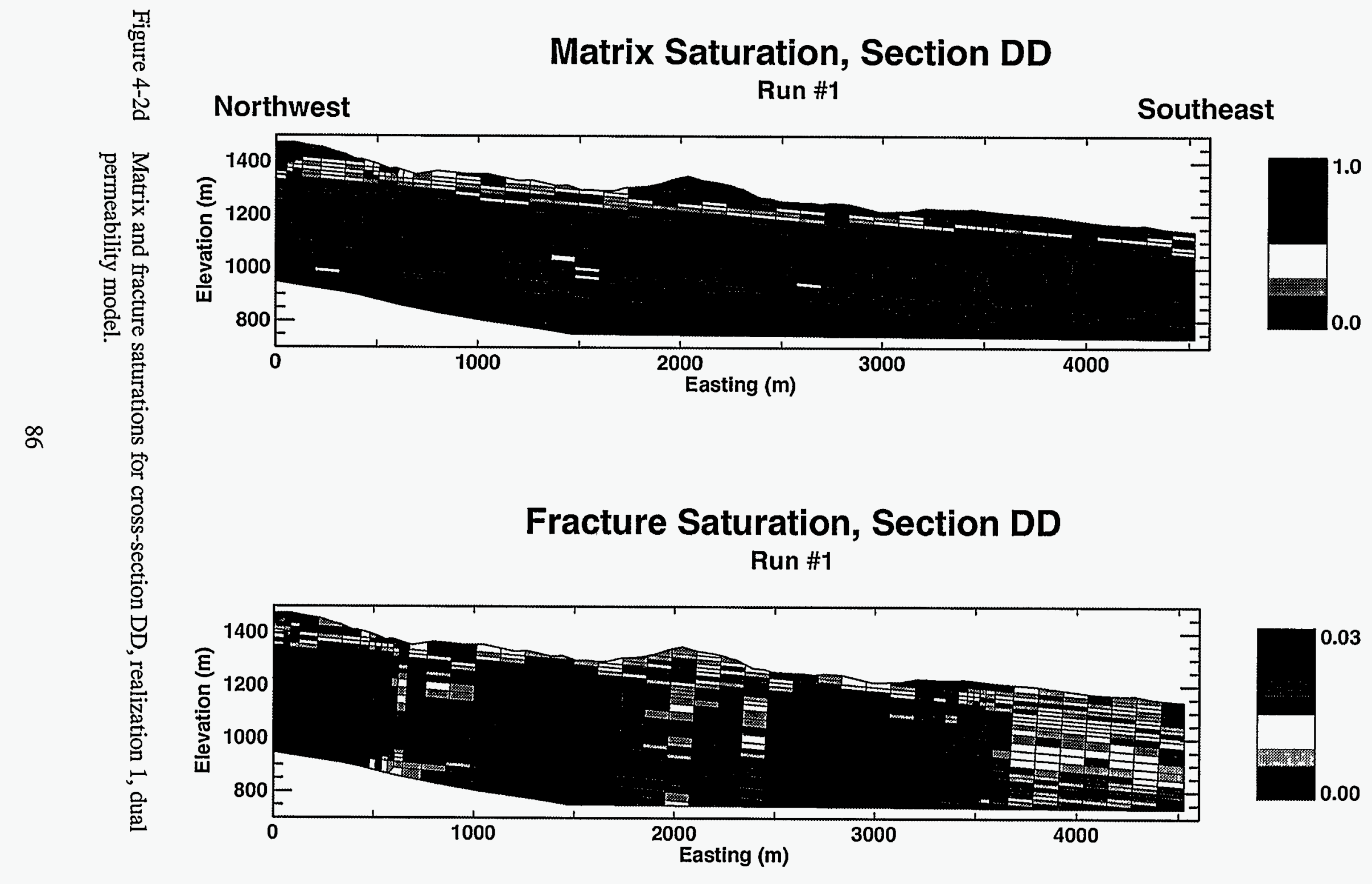


the infiltration flux for the two cross-sections (Figure 4-1) also shows that the infiltration is slightly higher at the location of the column of elements for cross-section DD (due to the interpolation) which could lead to higher saturations.

Other differences are observed at the intersection of cross-sections $\mathrm{BB}$ and $\mathrm{CC}$ at an elevation of approximately $800 \mathrm{~m}$ where the saturations simulated in cross-section BB are lower than those simulated in cross-section CC. Differences in the saturations can be explained by examining the saturated hydraulic conductivity in the elements where the differences are noted. The element in cross-section BB overlaps a portion of both the zeolitized Calico Hills and the non-zeolitized Prow Pass. This overlap results in an intermediate hydraulic conductivity between the two units being simulated for that element. This intermediate hydraulic conductivity is therefore higher than that simulated for the equivalent element in cross-section $\mathrm{CC}$ which resides more fully in the Calico Hills zeolitized unit. The higher simulated hydraulic conductivity in cross-section BB in turn results in a lower modeled saturation for that element.

A similar interpretation can explain the differences in saturations observed at intersection of cross-sections AA and CC at an elevation of approximately $1220 \mathrm{~m}$ (Figure 4-3). In this case the given elements have the properties of the PTn. Saturations simulated in cross-section CC are generally lower than those simulated in section AA. Examination of the porosity for the corresponding elements for the two cross-sections shows a higher porosity for cross-section CC. This element must almost fully contain the PTn 1 unit, which has a mean porosity of 0.46 , whereas the corresponding elements in cross-section AA overlaps either the PTn2 (the fifth element from the top) or TSw (6th element from the top) resulting in a lower porosity. The higher the porosity in the non-welded units the lower the saturation due to the increased pore space, thus explaining the discrepancies in saturations. Both of these cases of discrepancies in saturations between cross-section can most likely be resolved by using a finer grid. However, as in most locations the saturations match closely, the grid size seems adequate for simulating saturations.

As a method to evaluate whether the simulated matrix saturation values are reasonable, these saturations are compared to saturations measured in boreholes (Figure 4-4a to 4-4b). It should be noted that the core measurements are made on core plug samples on the order of $40 \mathrm{~cm}^{3}$ whereas the modeled saturations are simulated on grid blocks on the order of $1000 \mathrm{~m}^{2}$. Thus, this discrepancy in scale makes the comparisons less straight forward and accounts for the large variation in core measurement values. The boreholes with available saturation data that are closest to the individual cross-section locations are used. On cross-section AA, data from SD-9 (located approximately $600 \mathrm{~m}$ north of the cross-section) are used for comparison. Likewise, on cross-section BB data from UZ-16 (located approximately $150 \mathrm{~m}$ south of the cross-section) and SD-12 (located approximately $200 \mathrm{~m}$ north of the cross-section) are used. SD-9 and SD-12 intersect cross-section CC and NRG-6, NRG-7 and UZ-14 are used for comparisons in crosssection DD. Figure 3-1 presents the locations of the borings relative to the cross-sections.

GWTT-95 simulations are able to match measured core data saturations relatively closely (Figure 4-4a to 4-4b). Saturations in the Calico Hills non-welded, non-zeolitized units are well matched as shown in the comparison of the SD-12 data to the simulated saturations of crosssection CC and to a lesser extent, cross-section BB. The lower saturations in the PTn are also well matched by the simulated saturations of the four cross-sections. The large differences are in 
Intersection of Section A-A and C-C

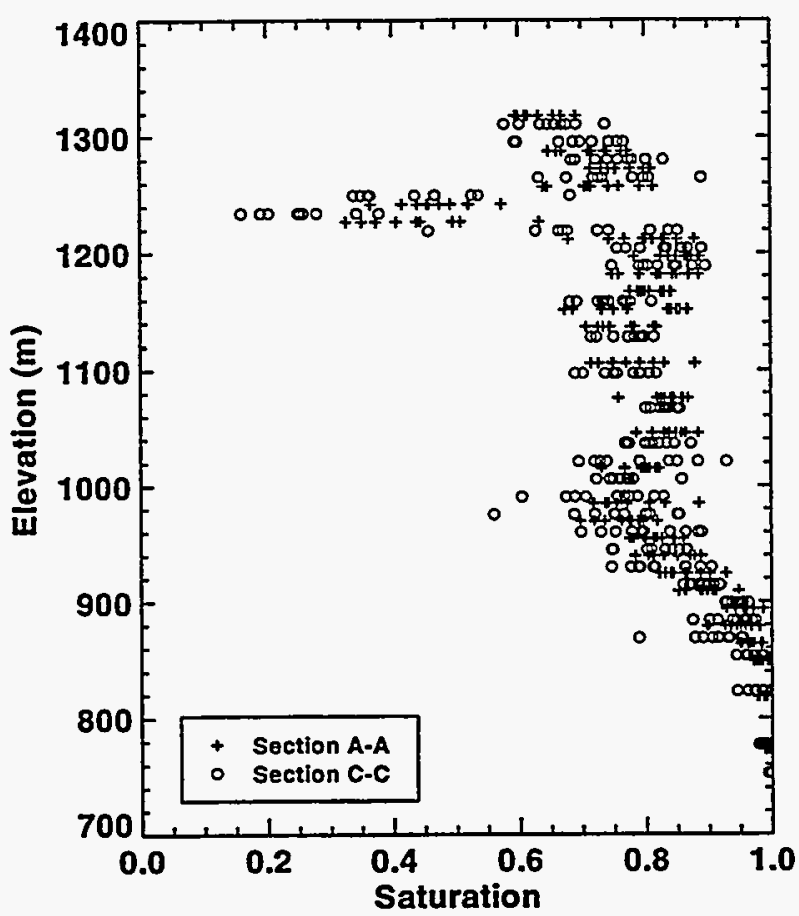

Intersection of Section B-B and C-C

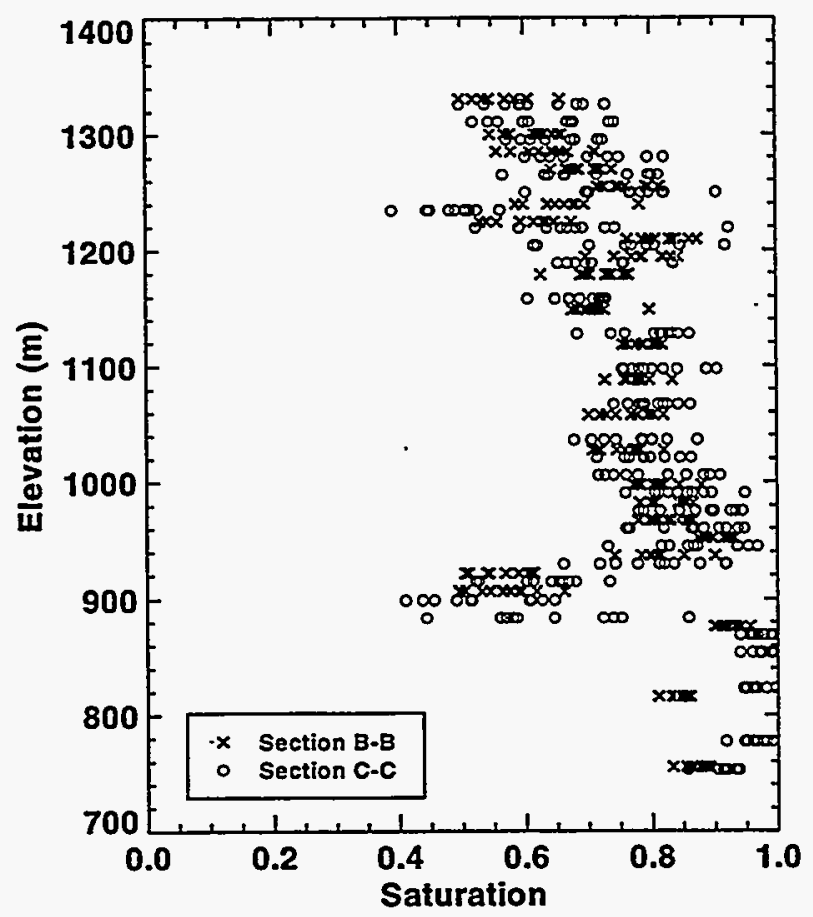

Intersection of Section A-A and D-D

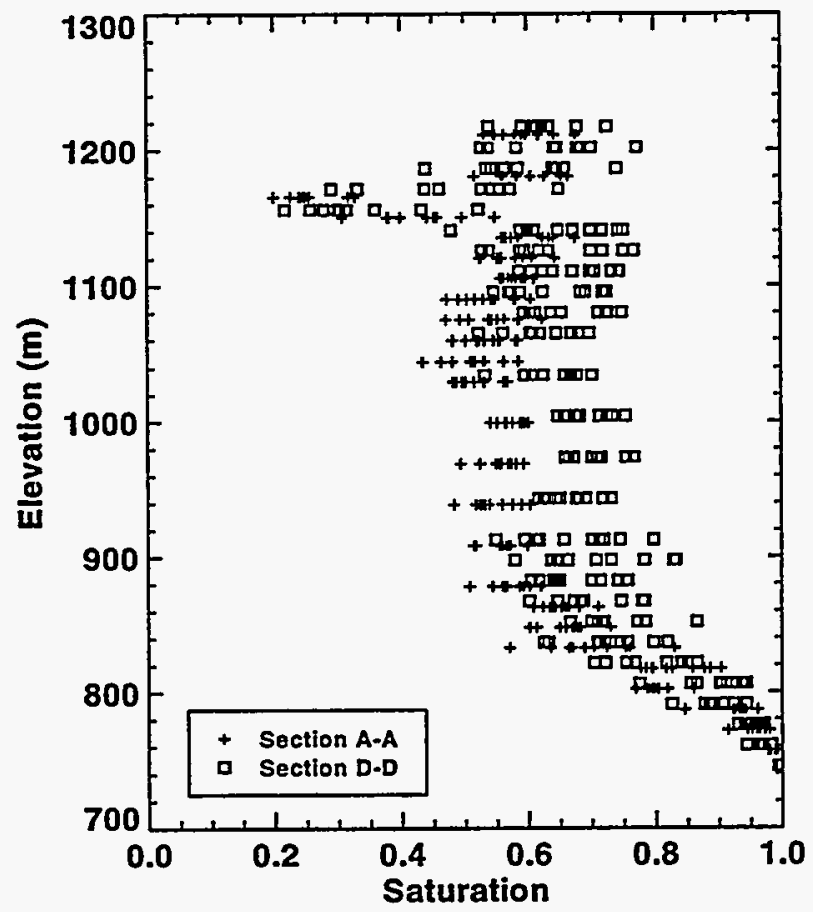

Intersection of Section C-C and D-D

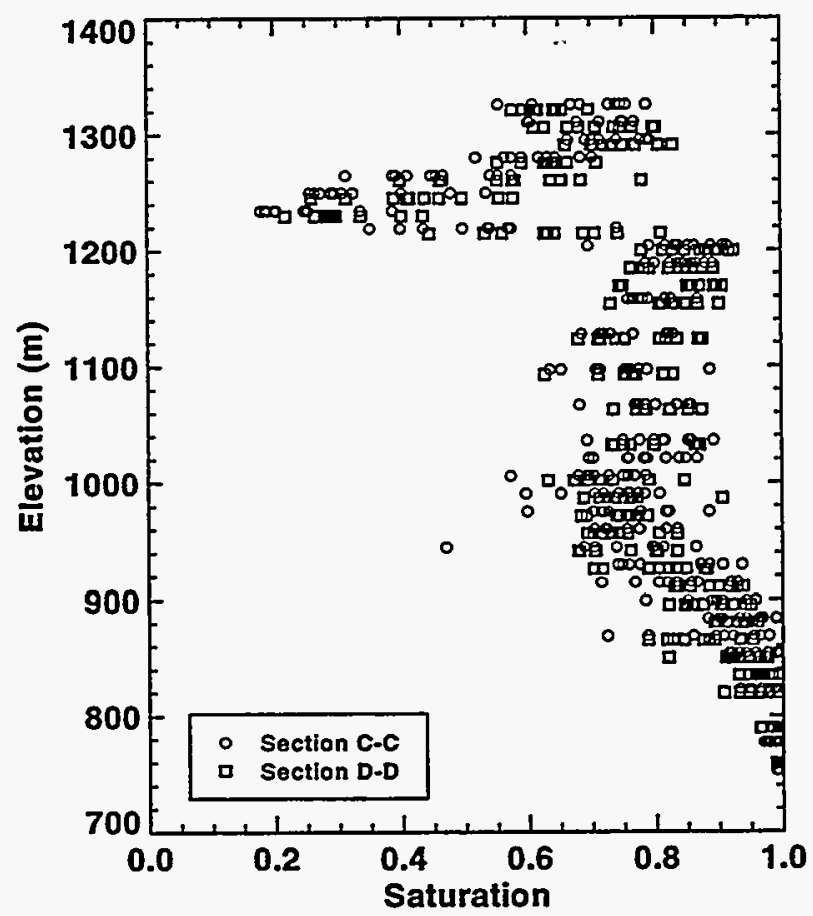

Figure 4-3 Simulated matrix saturations with depth at two columns of elements at the intersection of two cross-sections. 
Cross-Section A-A

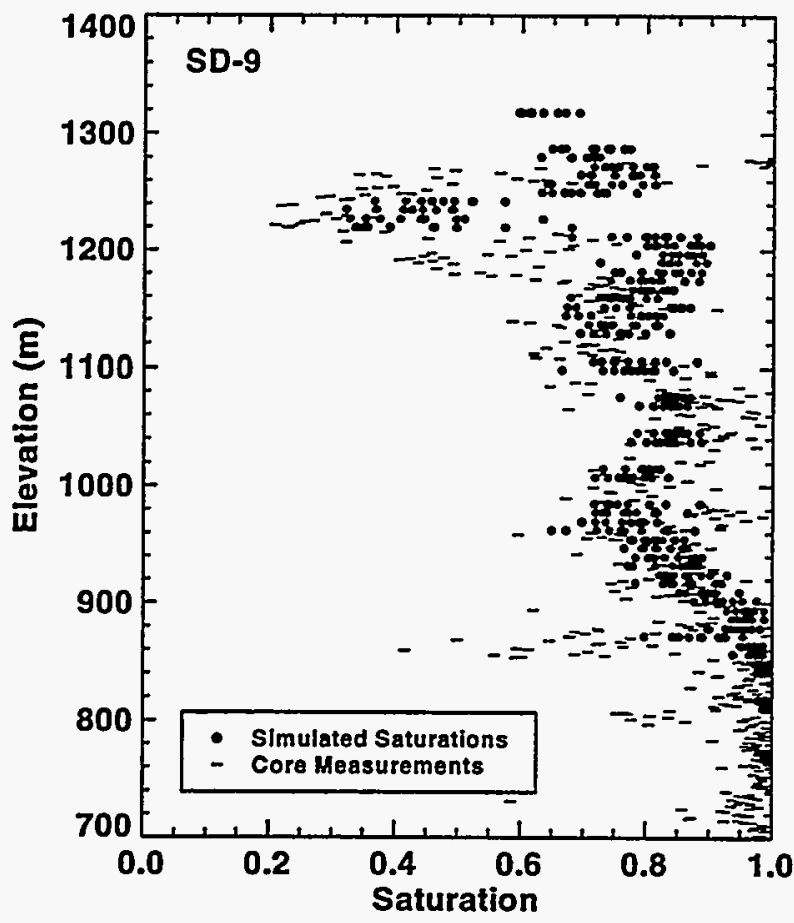

Cross-Section D-D

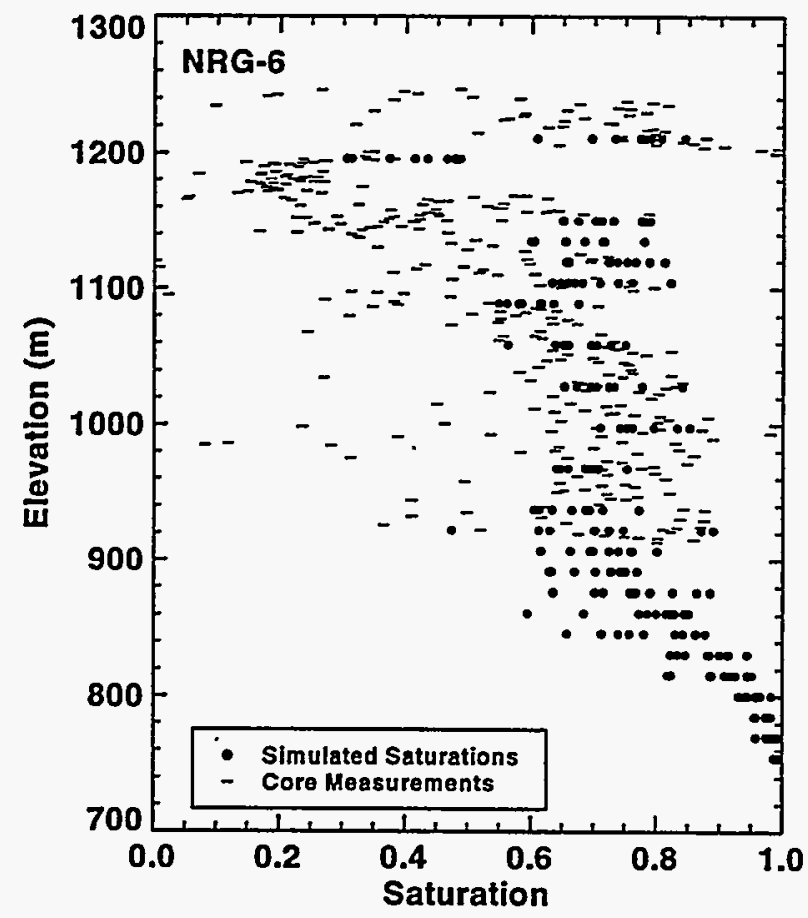

Cross-Section D-D

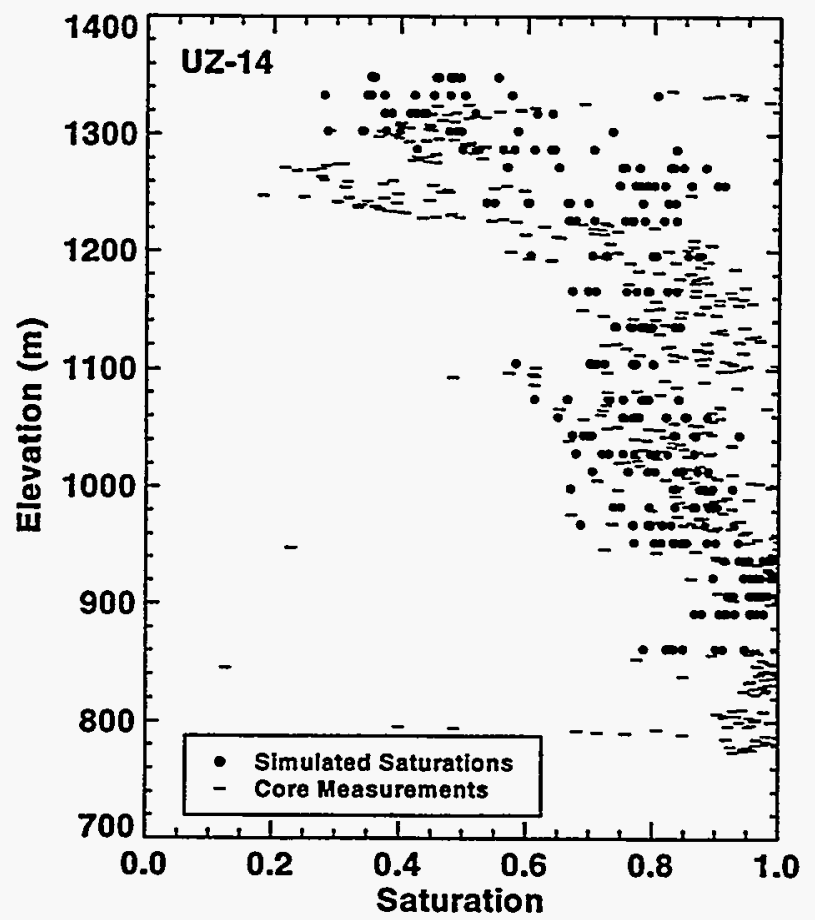

Cross-Section D-D

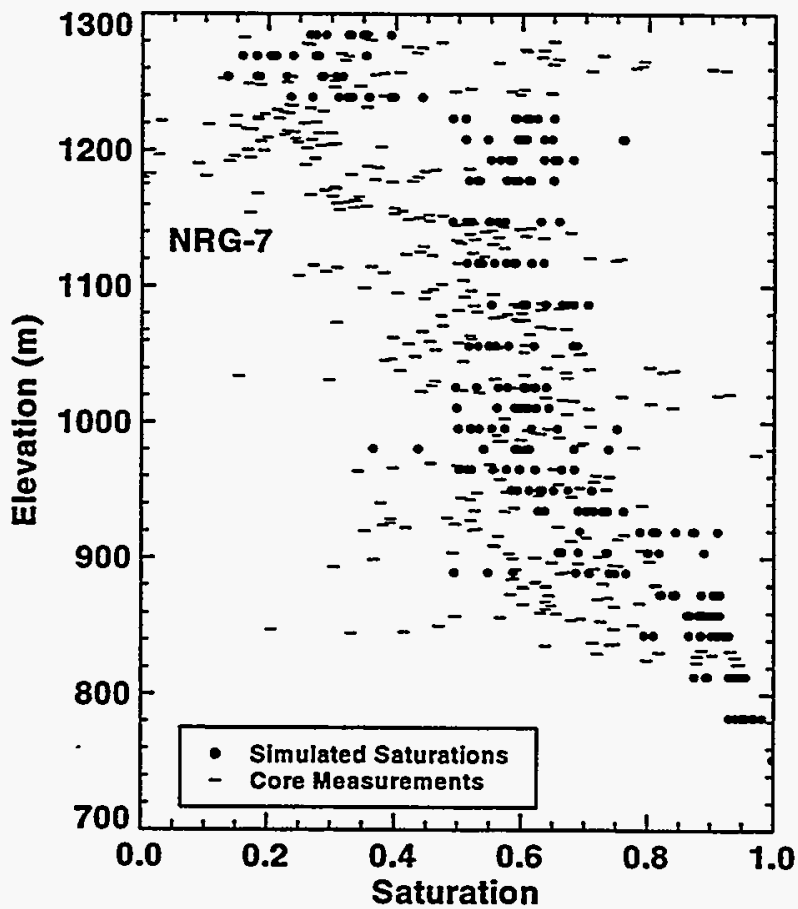

Figure 4-4a Comparisons of simulated matrix saturations to saturation measurements made from core samples from boreholes close to cross-sections AA and DD. 
Cross-Section B-B

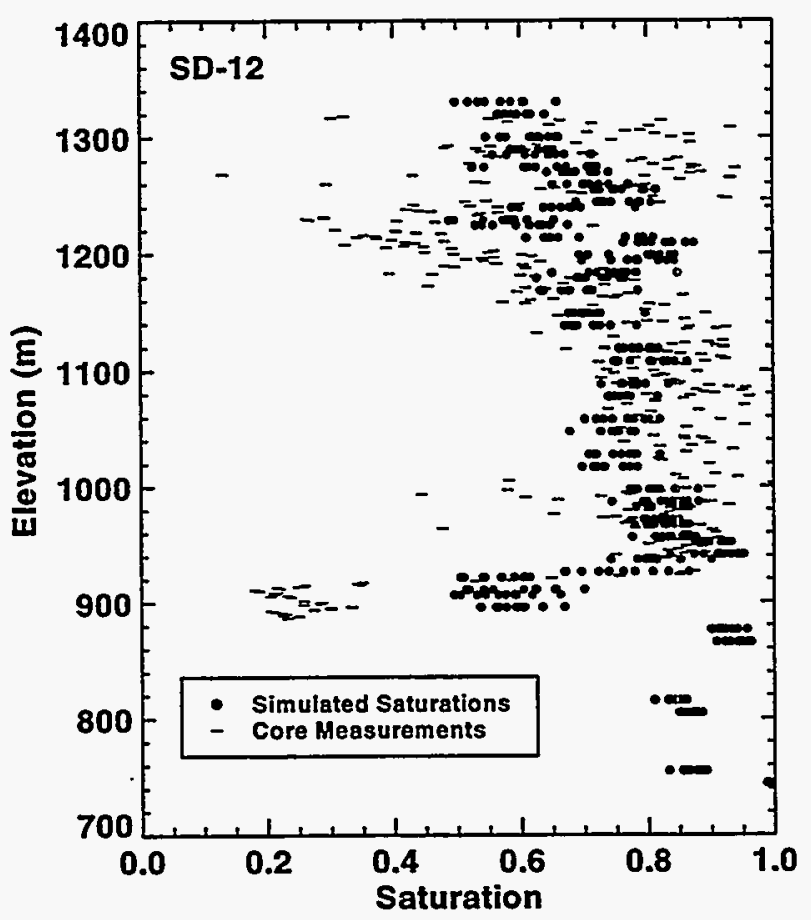

Cross-Section C-C

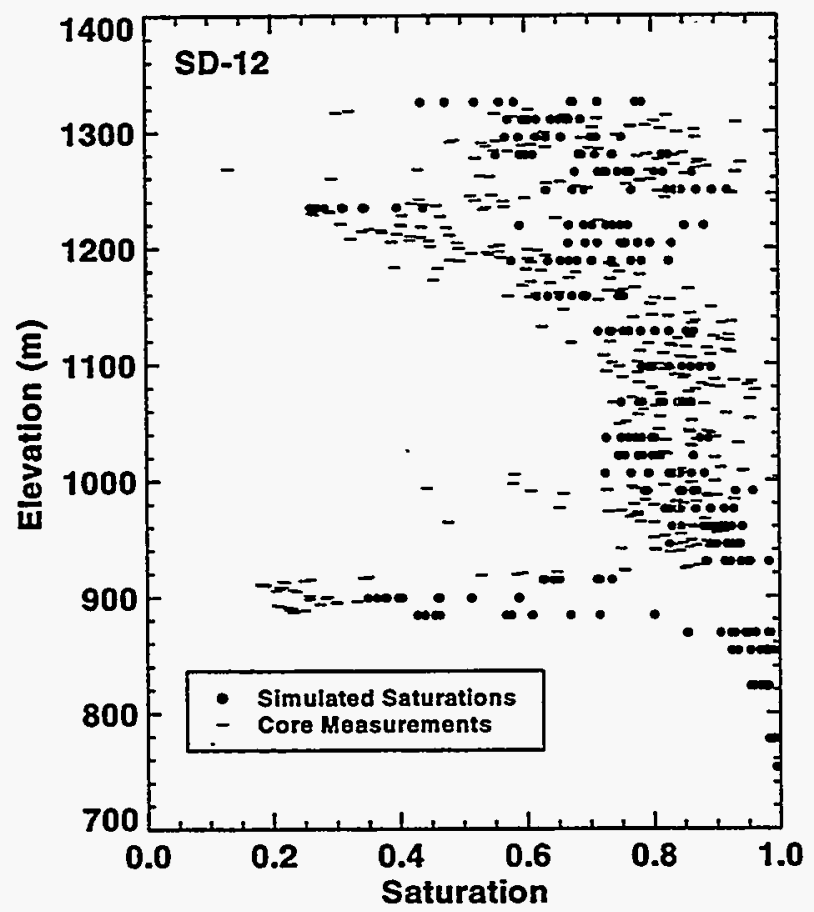

Cross-Section B-B

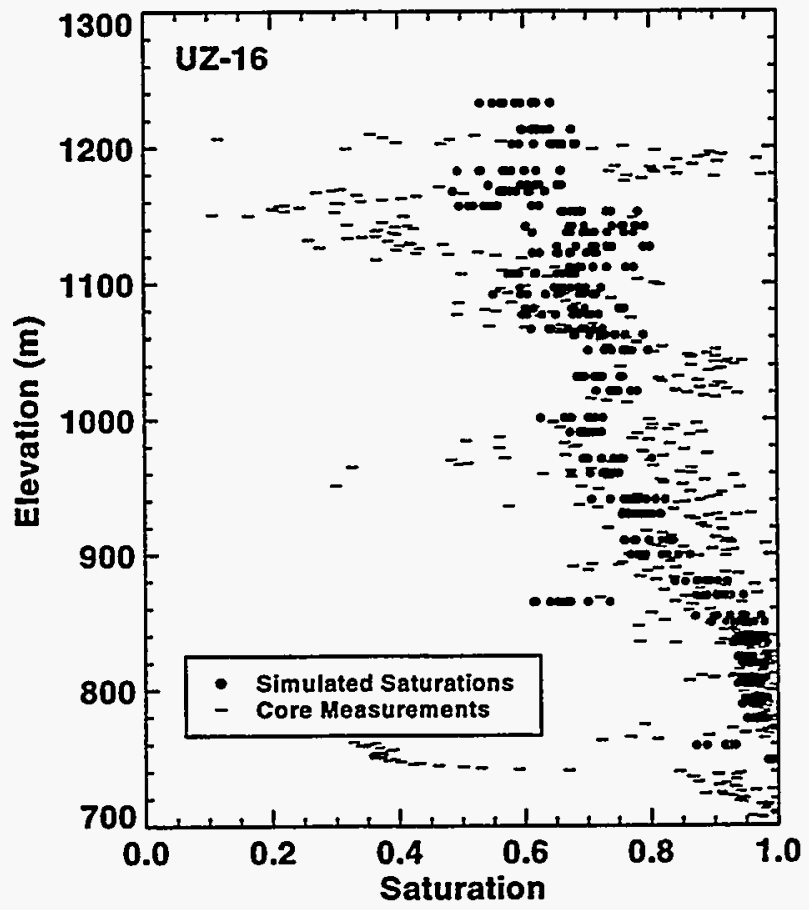

Cross-Section C-C

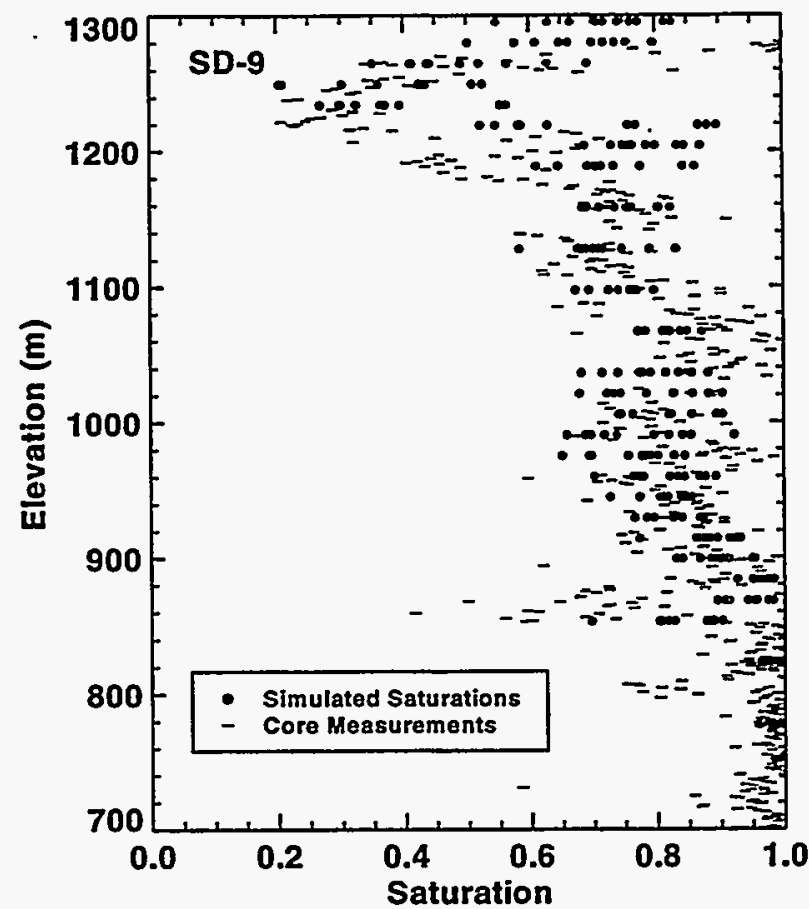

Figure 4-4b Comparisons of simulated matrix saturations to saturation measurements made from core samples from boreholes close to cross-sections BB and CC. 
the TCw. The difference can be explained by the use of the DKM for GWTT-95 (also see Section 4.5.7). Infiltration is initiated only into the fracture domain and much of the fluid remains in the fractures as it flows through the TCw. It is not until the fluid enters the PTn that much of it is imbibed into the matrix. To better match the saturations in the TCW using the DKM some of the infiltration could be introduced into the matrix domain along the upper boundary.

Also of interest, when interpreting the matrix saturation results is how much the simulated saturations will vary among the geostatistical simulations. In other words, how much do the simulated saturations vary due to uncertainty in the hydrogeological parameters? Examination of Figures 4-3, 4-4a and 4-4b show a range of 5-25\% in simulated saturations among geostatistical realizations. On average there appears to be a range of approximately $10 \%$. It is expected that this amount of variation should significantly affect the flow through the system and, therefore, the resulting particle travel times.

\subsubsection{Fracture Saturations}

Saturation in the fractures are much lower than in the matrix with ranges from the residual saturation of 0.0001 to 0.03 (Figure 4-2a to 4-2d). Zones of relatively lower fracture saturations correspond to those of relatively lower matrix saturations and areas of lower infiltration. The fracture domain is generally less saturated in vitrophyres and more saturated in the PTn. These results are most likely due to the inverse relationship between pore space and saturation as the vitrophyres have a relatively high fracture porosity and the PTn a relatively low fracture porosity. The random areas of higher fracture frequency (see Section 3.3.3) do not have a great effect on the fracture saturations.

\subsubsection{Flow Velocities}

Flow velocities in both the matrix and fractures are predominantly vertical in the four cross-sections (Figure 4-5a to 4-5b). The magnitude of the Darcy velocities ranges from approximately $10^{-13}$ to $10^{-9} \mathrm{~m} / \mathrm{s}$ in the matrix domain with the majority of the area having a velocity of between $10^{-12}$ and $10^{-10} \mathrm{~m} / \mathrm{s}$ (Figure 4-6a to 4-6b). Velocities are lower where infiltration is low. In the fracture domain velocities range from approximately $10^{-13}$ to $10^{-5} \mathrm{~m} / \mathrm{s}$ with the majority of the area having a velocity of between $10^{-11}$ and $10^{-6} \mathrm{~m} / \mathrm{s}$. Again, velocities are lower where infiltration is low. The velocities also tend to decrease with depth. Based on a comparison between the range of Darcy velocities in the fracture and matrix domains, in the majority of elements fluid flow in the fracture domain is faster than in that of the matrix. These observations-are confirmed with the particle tracking (see Section 4.4).

Through most of the system there is flow from the fractures to the matrix (Figures 4-7a to 4-7b). Water is simulated to flow from the matrix to the fractures mostly near the water table or in the zeolitic regions where matrix is generally close to full saturation. These observations are not surprising as it is only where the matrix is almost completely saturated that the capillary pressure decreases to below that of the fractures. The simulated relative flux through the fracture and matrix continua is shown in Figure 4-8. A very high fraction of the flow occurs in the fractures in 


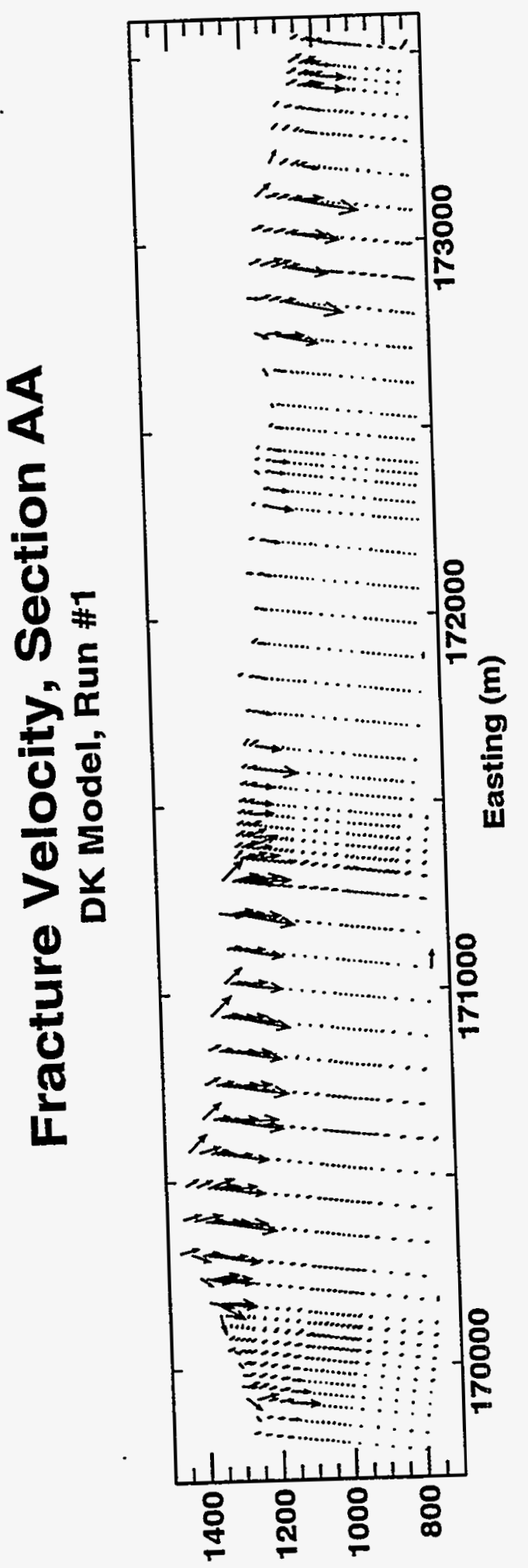

(u) Uoำen키코

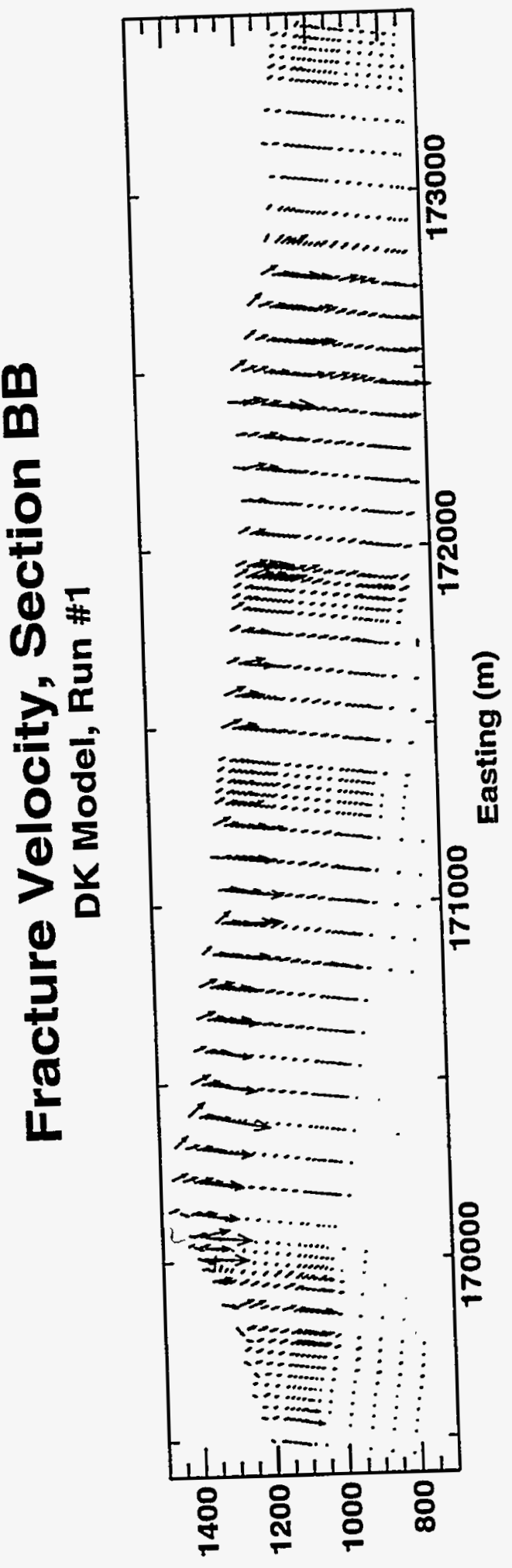

(u) Uоำยก키

Figure 4-5a Velocity vectors of flow through the fracture continuum for cross-sections $\mathrm{AA}$ and $\mathrm{BB}$, realization 1, dual permeability model. All vectors in Figure 4-5 (cross-sections $\mathrm{AA}, \mathrm{BB}, \mathrm{CC}$, and DD) have the same scale. 


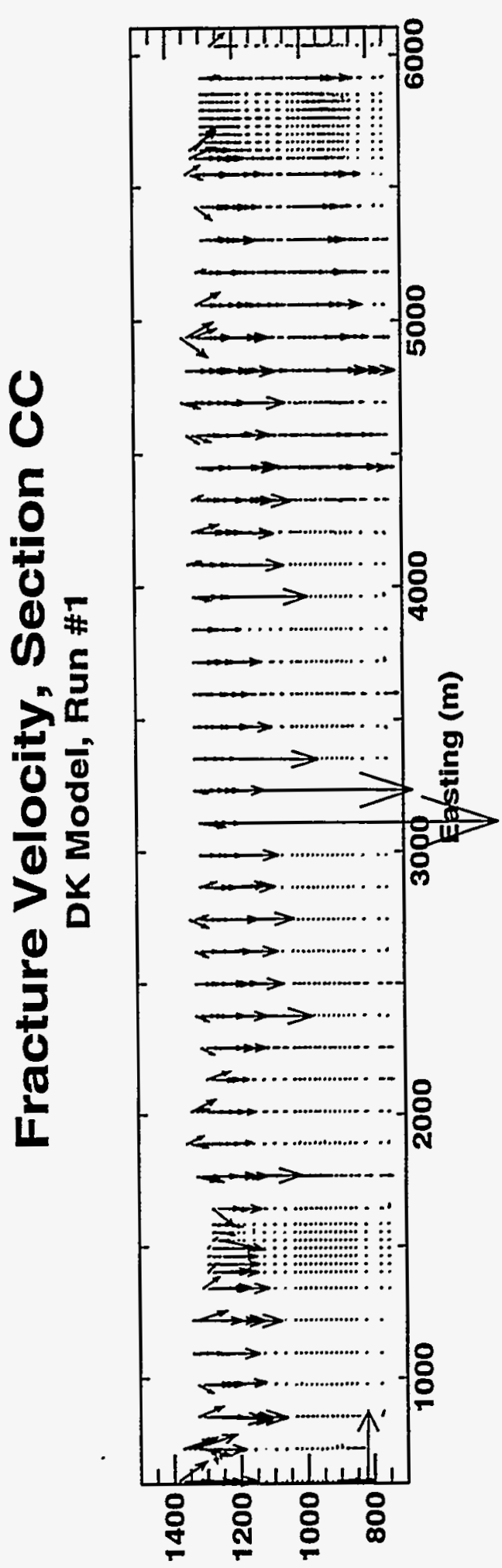

(w) นо!̣еләәコ

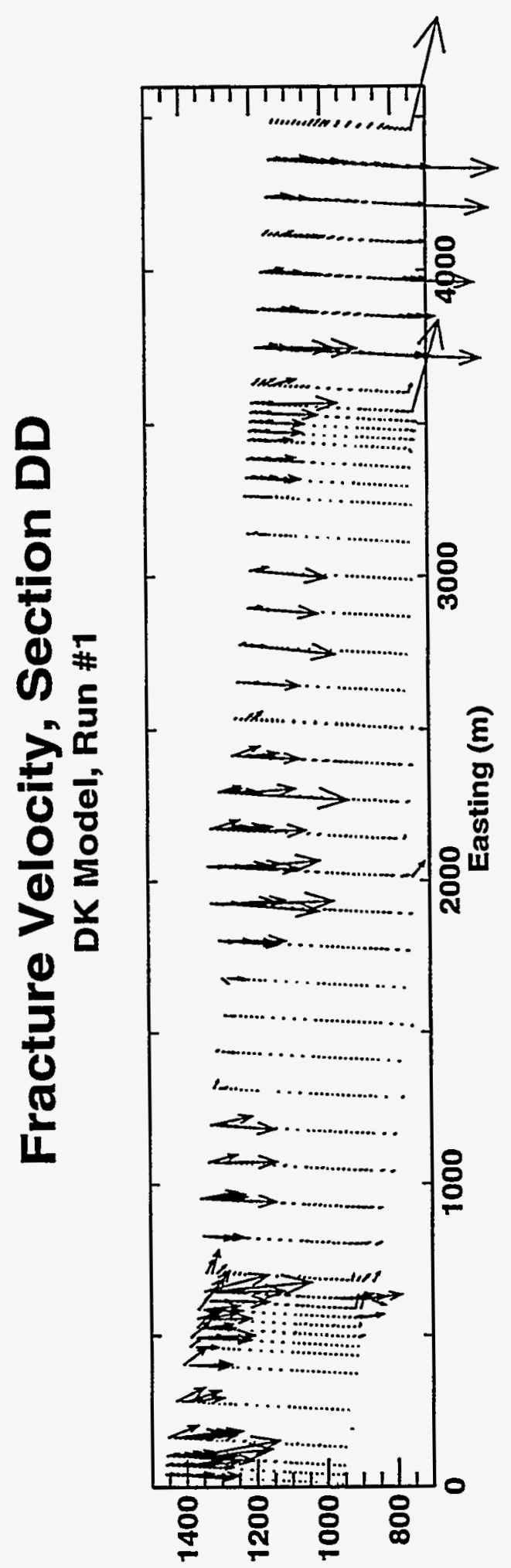

(u) uo!̣enәəコ

Figure 4-5b Velocity vectors of flow through the fracture continuum for cross-sections $\mathrm{CC}$ and DD, realization 1, dual permeability model. All vectors in Figure 4-5 (cross-sections AA, BB, CC, and DD) have the same scale. 

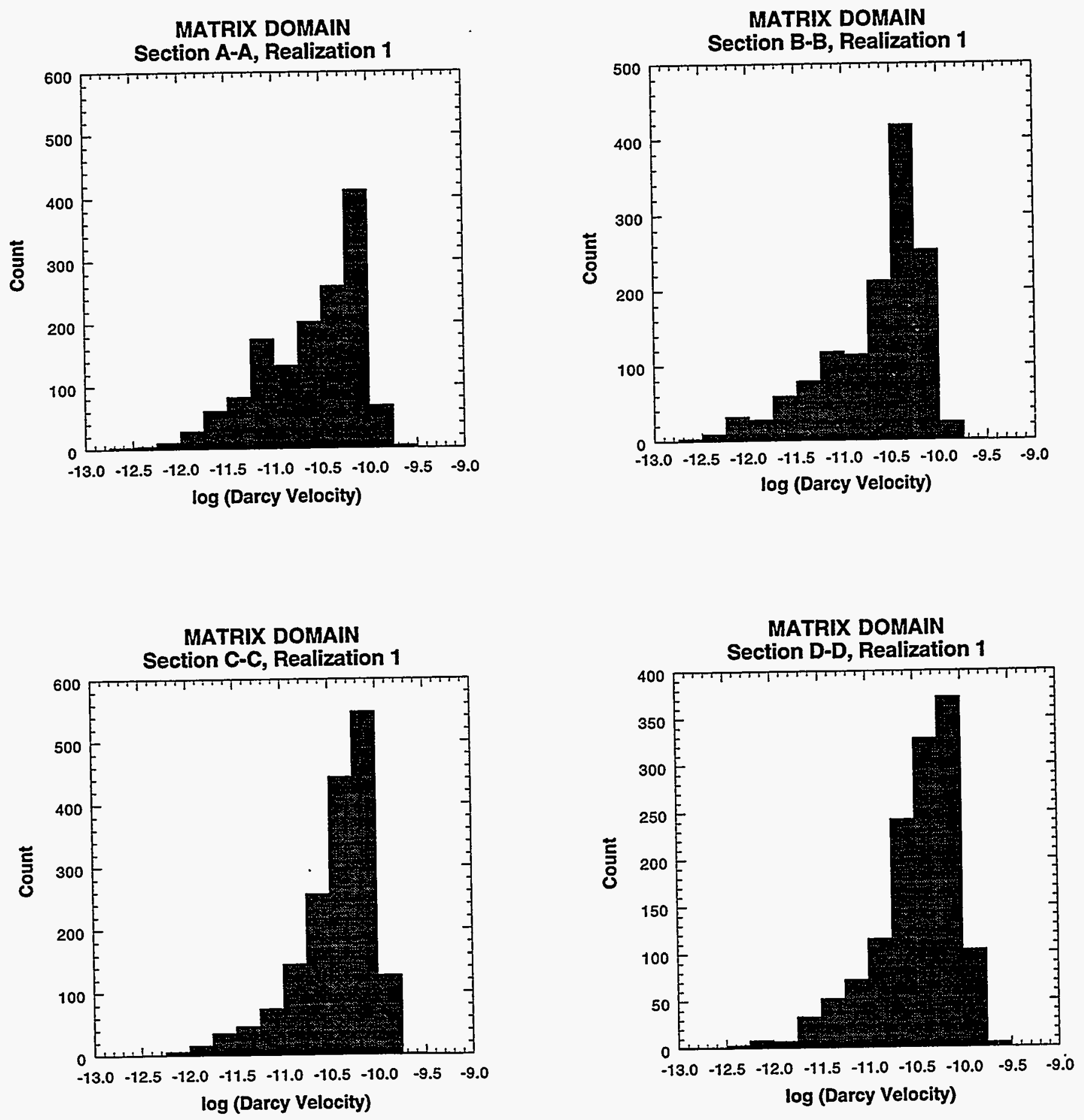

Figure 4-6a Distribution of Darcy velocities through the matrix domain for one realization of all four cross-sections. 
FRACTURE DOMAIN

Section A-A, Realization 1

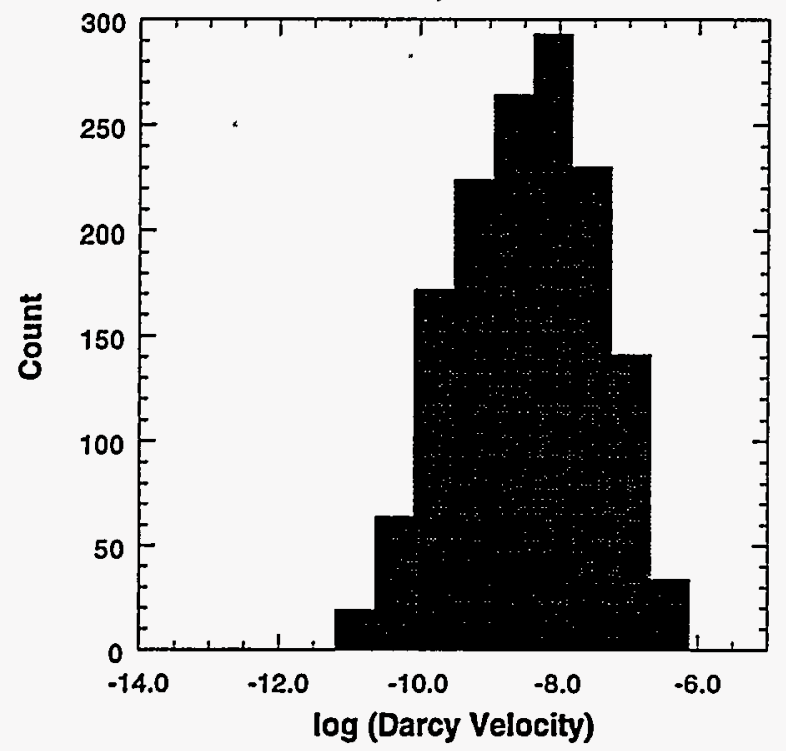

FRACTURE DOMAIN

Section C.C, Realization 1

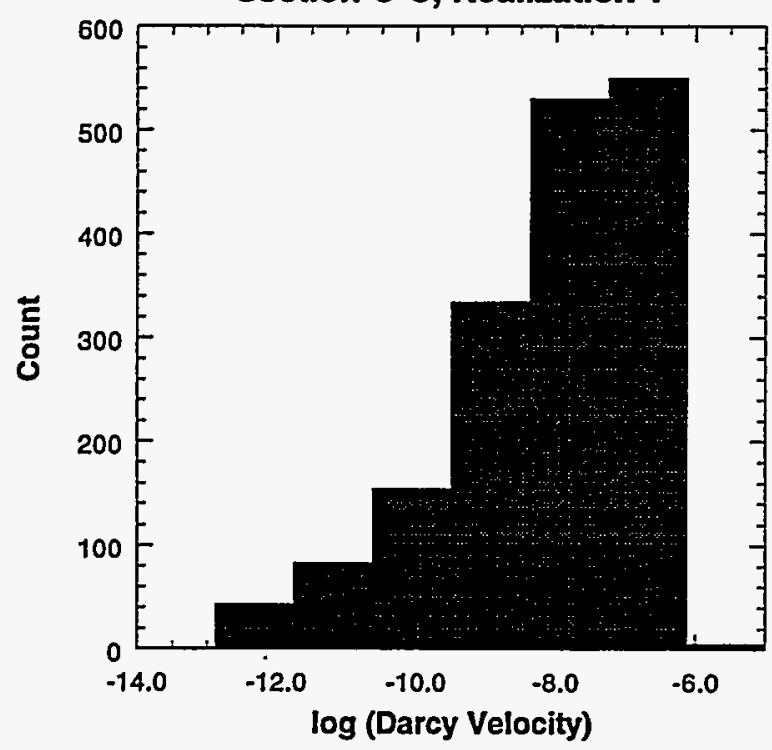

FRACTURE DOMAIN

Section B-B, Realization 1

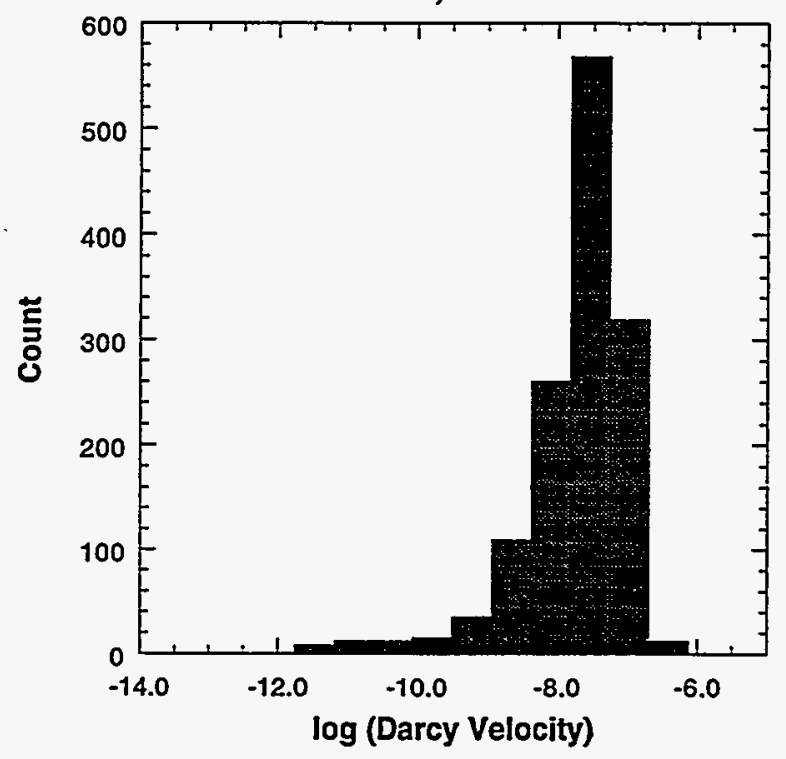

FRACTURE DOMAIN Section D-D, Realization 1

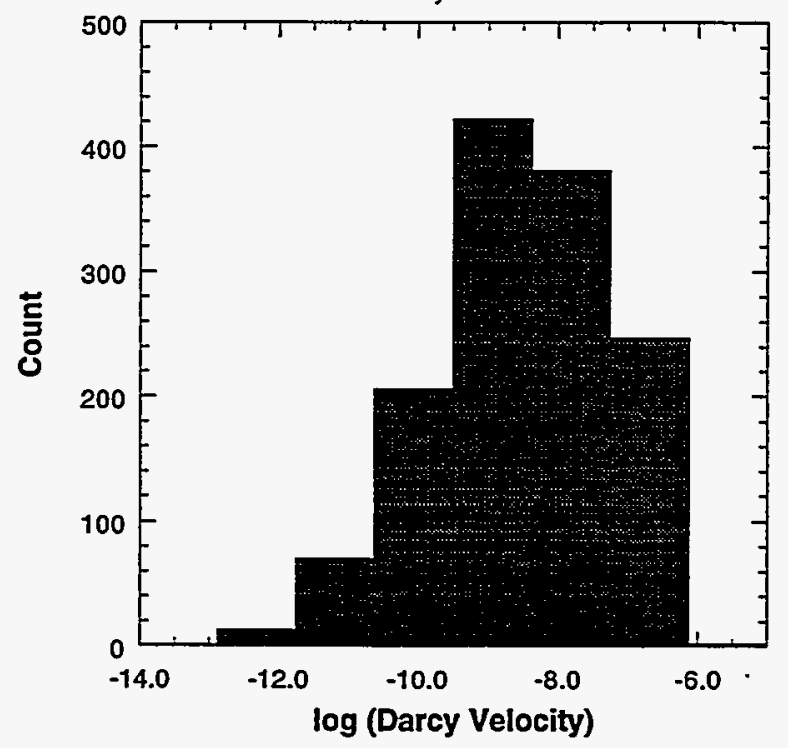

Figure 4-6b Distribution of Darcy velocities through the fracture domain for one realization of all four cross-sections. 

Section AA, DK Model, Run \#1

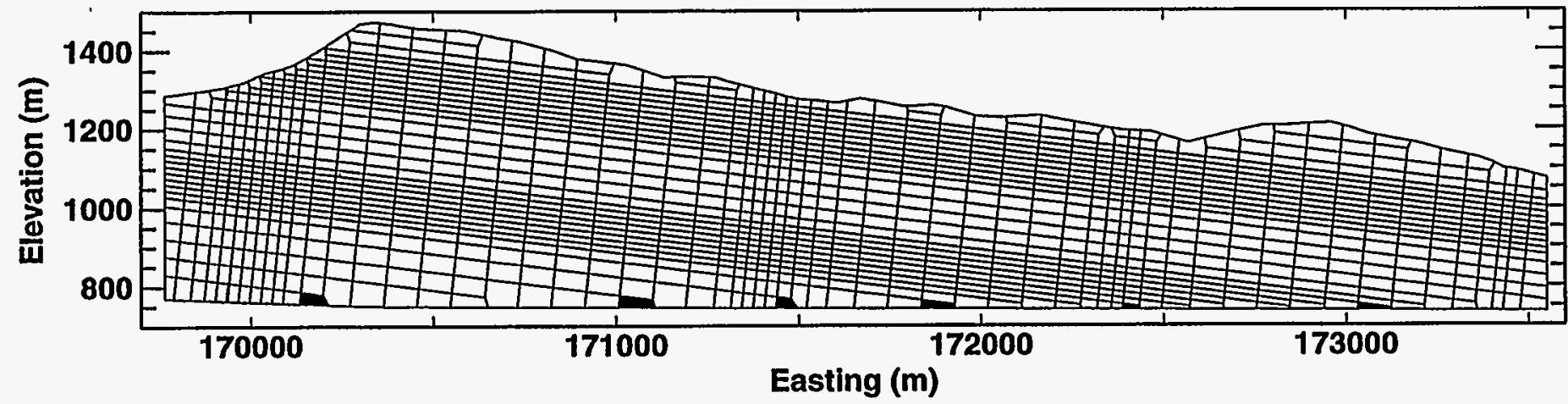

$\square$ Fracture to Matrix

Matrix to Fracture

\section{Flow Between Fractures and Matrix} Section BB, DK Model, Run \#1

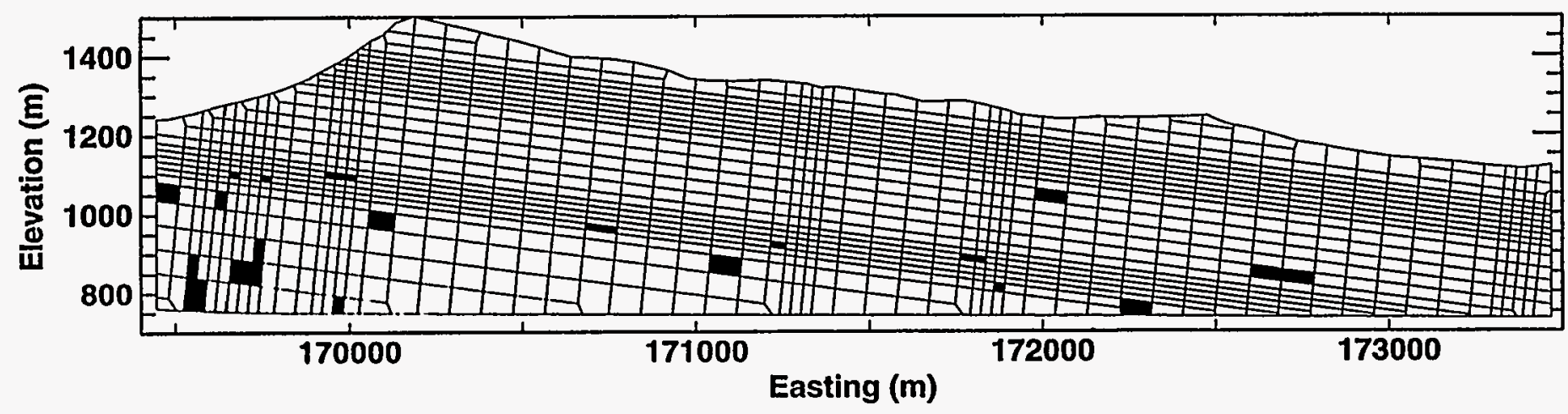

$\square$ Fracture to Matrix

Matrix to Fracture 

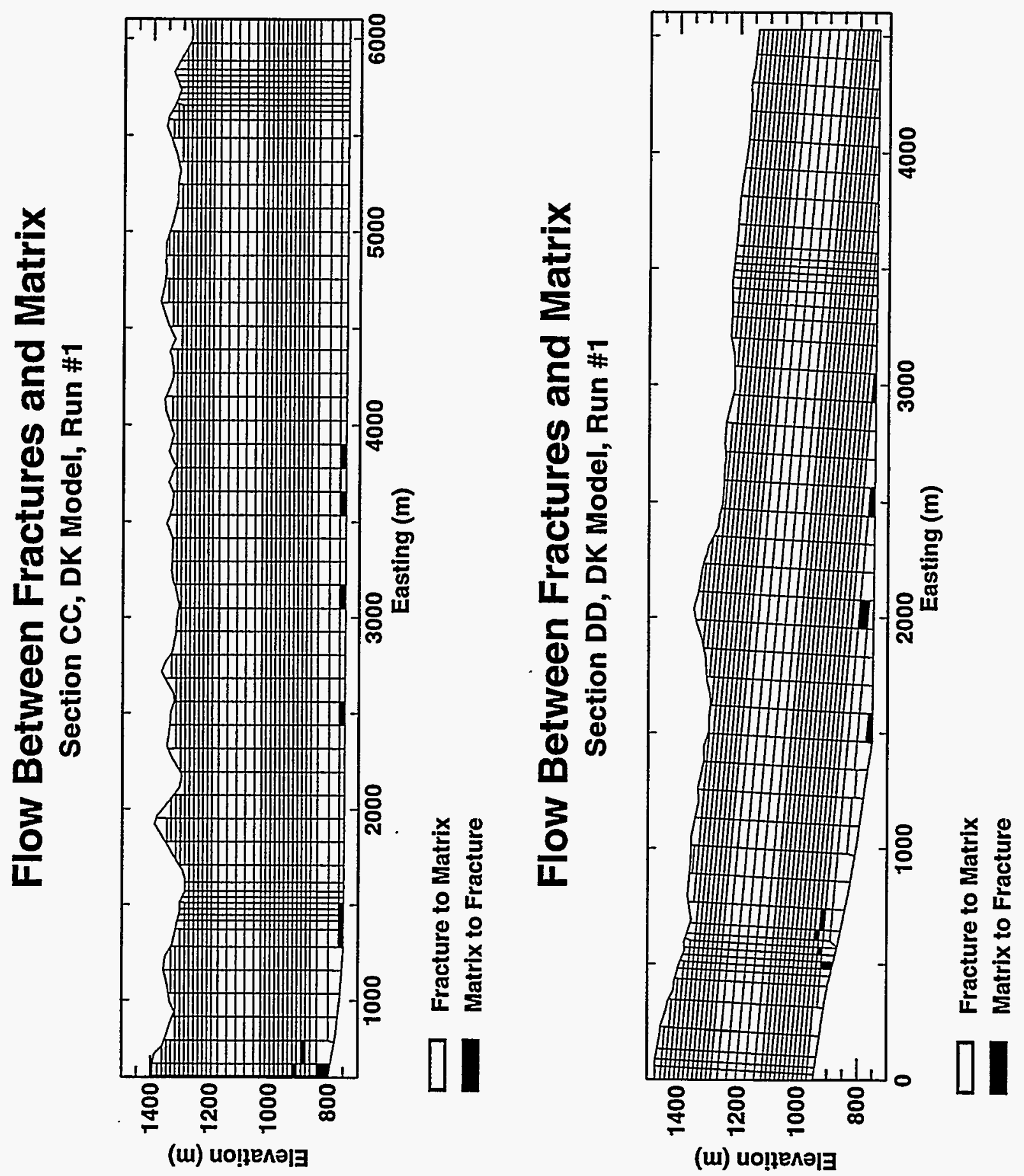

Figure 4-7b Flow between matrix and fracture continuums for cross-sections $\mathrm{CC}$ and $\mathrm{DD}$, realization 1 , dual permeability model. 
This page intentionally left blank. 
the $\mathrm{TCW}$ unit and the zeolitized regions. The fraction of flow in fractures in the area below the region of low infiltration in the center of cross-section AA is significantly lower than in the rest of the section.

\section{fracture flux/total flux}

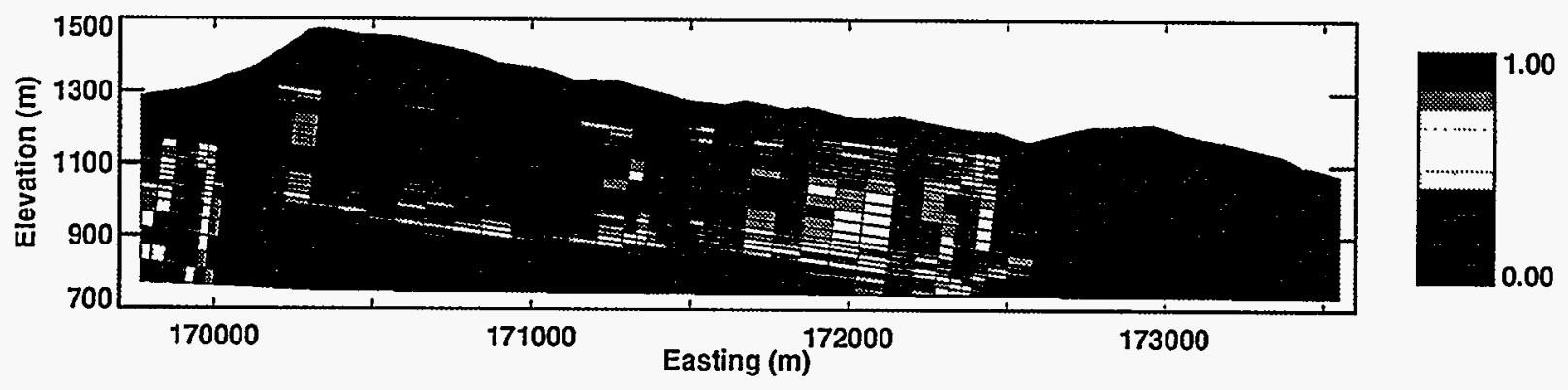

Figure 4-8 Ratio of fracture flux to total flux in the flow grid for the dual permeability simulation on cross-section AA, realization 1.

\subsection{Particle Tracking}

A particle tracking algorithm is employed to simulate the movement of water particles in the steady-state flow field in the unsaturated zone calculated by TOUGH2. Multiple particles are tracked from the repository horizon in each of the cross-sections to the water table and the travel time for each particle is noted. The particle tracking method is consistent with the dualpermeability conceptual model of flow, in that particles may be moved by flow in the fracture continuum or by flow in the matrix. A conservative (in the sense of repository performance) approximation of the interaction of groundwater flow between fractures and matrix is used to determine in which continuum the particle is moved.

\subsubsection{Particle Transport Processes}

Water particles are transported by advection and hydrodynamic dispersion in the liquid phase. Vapor phase movement of water particles is not considered. Steady state advection occurs at the local fluid velocity in either the fracture or matrix continuum. Dispersion of particles occurs by a random Fickian process due to heterogeneity of the media at the sub-numericalelement scale and due to molecular diffusion.

Advective movement of particles occurs at the faster of the velocities in the fracture or matrix continua. The particle is thus retained in the fracture continuum as long as the fracture flow velocity exceeds the corresponding matrix flow velocity at the current location of the particle. If the particle is flowing at the matrix flow velocity it is immediately transferred to the fracture continuum if the fracture flow velocity becomes greater. This simplified, but 
conservative, conceptualization of fracture-matrix interaction calculates the fastest pathways in the unsaturated flow system. However, it does not simulate the complete distribution of particle travel times within the flow field, which should be considered when interpreting the results of the simulations.

\subsubsection{Implementation of Particle Tracking}

Particle tracking is performed within the steady-state unsaturated flow field calculated by TOUGH2. A post-processing procedure is used to calculate the average groundwater velocity for both fractures and matrix within each cell in the TOUGH2 mesh. These average velocities are based on the geometry of the mesh cell, porosities, the fluxes at element faces, and the principle of conservation of fluid mass (see Section 4.2.1).

The particle tracking method used is based on the semianalytical method of Lu (1994). A code based on this method was obtained from the USGS and modified for use with dual continuum flow simulations and to simulate dispersion. Because the numerical mesh used in TOUGH2 flow simulations is unstructured and the semianalytical method of advective particle tracking requires fluid velocities on a regular, orthogonal grid, the method first calculates groundwater velocities on a regular grid by interpolation. The interpolation technique uses the nearest simulated velocities in each of the quadrants surrounding the point to be estimated and calculates the velocity by inverse-distance-squared interpolation. This interpolation step has the disadvantage of possibly "averaging out" anomalously high or low values of velocity.

The process of hydrodynamic dispersion is simulated in the particle tracking procedure by the random-walk method. Following each time step the particle is displaced a random distance in both the longitudinal and transverse directions. The additional displacement is randomly drawn from a Gaussian distribution with the variance defined as:

$$
\sigma^{2}=2\left(v \alpha_{i}+D_{m}\right) \Delta t
$$

where $v$ is pore velocity, $\alpha_{i}$ is the dispersivity (longitudinal or transverse); $D_{m}$ is the effective coefficient of molecular diffusion, and $\Delta t$ is the time step. It is assumed that the dispersive process is the same in both the fracture and matrix continua and due to lack of data, the same values of $\alpha$ and $D_{m}$ are used in the fracture and matrix domains. A value of $9.3 \times 10^{-5} \mathrm{~cm}^{2} / \mathrm{s}$ is used for the effective coefficient of molecular diffusion. Longitudinal dispersivity is $10 \mathrm{~m}$ and transverse dispersivity is $1 \mathrm{~m}$ for all $\mathrm{UZ}$ simulations. This value for longitudinal dispersivity is less than the average theoretical value calculated for the fracture network for the TSw (see Section 3.2.2.5) because asymptotic macrodispersive behavior would probably not be achieved over the distance from the repository to the water table. The value of $10 \mathrm{~m}$ for longitudinal dispersivity in the matrix is probably excessive, but the focus of the groundwater travel time analysis is on the fast-path flow that occurs in the fracture continuum. 


\subsubsection{Particle Tracking Results}

Particle tracking results for the UZ flow simulations are summarized in Figures 4-9a to 49d. Each figure shows the infiltration rate applied to the upper boundary of the flow model, an example of the particle paths in one realization of the material properties for the cross-section, and the distribution of particle travel times for the ten realizations of the cross-section. The spatial distribution of infiltration for each cross-section reflects the trend of lower infiltration in washes and higher infiltration on side slopes and ridge tops (see Section 4.1). Particle paths are primarily vertical downward in each of the cross-sections, with some minor lateral advection near the water table. The random dispersive component of particle movement is also apparent in the plots of particle paths. Movement of particles at fracture flow velocities is dominant in most regions traversed by particles in the examples shown for the four cross-sections. There are some areas apparent in the four cross-sections (shown as red pathlines) in which matrix flow velocities exceed fracture flow velocities.

The distributions of particle travel times from the repository horizon to the water table are shown for all ten realizations of each cross-section in Figures 4-9a to 4-9d. There are relatively broad distributions of travel times for any given location along the cross-sections as well as considerable variability in travel times along the lengths of the cross-sections. Random dispersion of particle movement and differing material properties in the multiple geostatistical realizations of the system both contribute to the variability of travel times at a given location. The travel times for any given realization are plotted as a single color/symbol on the graphs in Figures 4-9a to 49d. Careful examination of these figures shows that there is generally greater variability in travel time among geostatistical realizations than there is due to dispersion within a single realization. There is a general inverse correlation between infiltration and average travel time, as expected. Particles which experience significant periods of flow at matrix flow velocities have much longer travel times, as shown in Figure 4-9d for cross-section DD.

Most simulated particle travel times along the four cross-sections exceed 1,000 years. A few particles at the southeastern end of cross-section DD travel to the water table in less than 1,000 years. A significant minority of particles have travel times of less than 1,000 years along the western portion of cross-section AA, the eastern and central portions of cross-section $B B$, and the northern portion of cross-section CC. Most of the particles at the southern end of crosssection CC travel to the water table in less than 1,000 years. The relatively fast movement of particles calculated for the southern part of cross-section CC is apparently the result of somewhat higher infiltration along this part of the cross-section coupled to significant thinning of the PTn unit in this area (see Section 3.1.1). The thickness of the PTn unit has a significant impact on the flux (and flow velocities) within the fracture continuum in the underlying TSw unit because of the diversion of flow from fractures to matrix that occurs in the PTn. Thinning of the PTn across the southern end of the repository could thus have an impact on the amount of fracture flow sustained from the surface to the repository horizon and below, resulting in relatively higher fracture flow velocities and shorter travel times. 

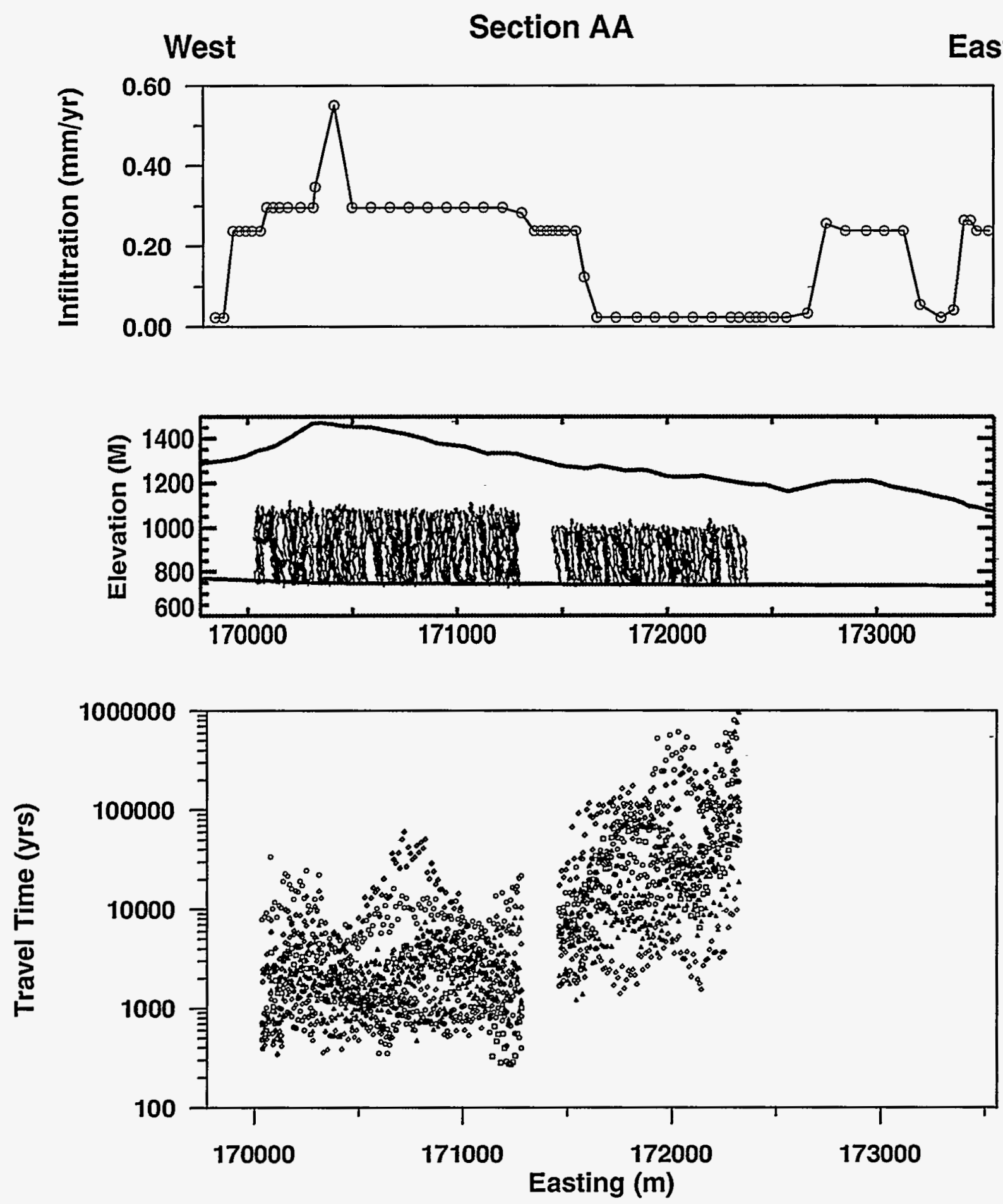

Figure 4-9a Infiltration, simulated particle paths and groundwater travel times along crosssection AA. Particle paths for a single realization of material properties are plotted in the middle figure. Portions of particle paths in which particles are advected at fracture flow velocities are plotted in blue and where particles are advected at matrix flow velocities paths are plotted in red. Travel times are plotted in the lower graph for all ten realizations. 

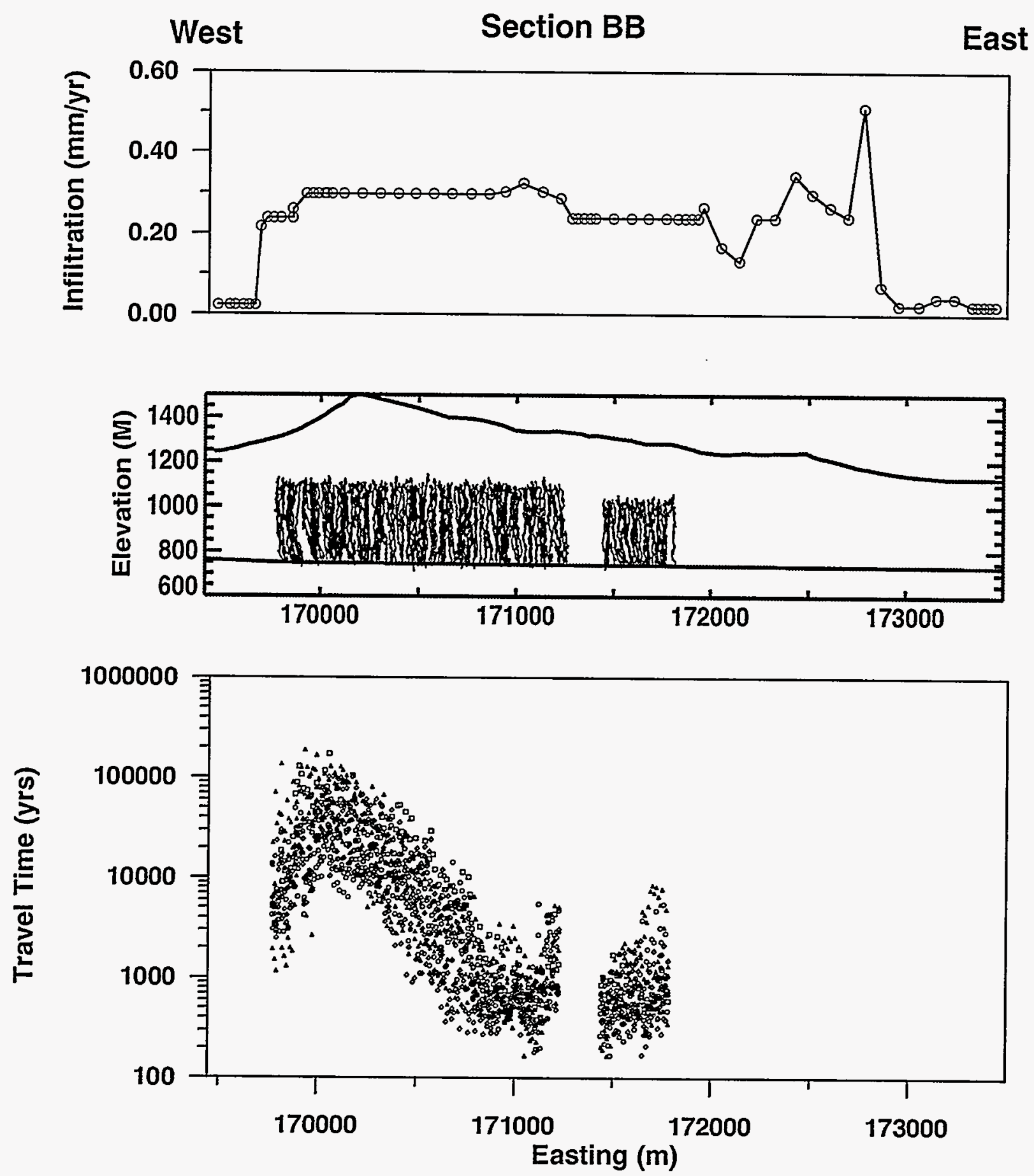

Figure 4-9b Infiltration, simulated particle paths and groundwater travel times along crosssection BB. Particle paths for a single realization of material properties are plotted in the middle figure. Portions of particle paths in which particles are advected at fracture flow velocities are plotted in blue and where particles are advected at matrix flow velocities paths are plotted in red. Travel times are plotted in the lower graph for all ten realizations. 

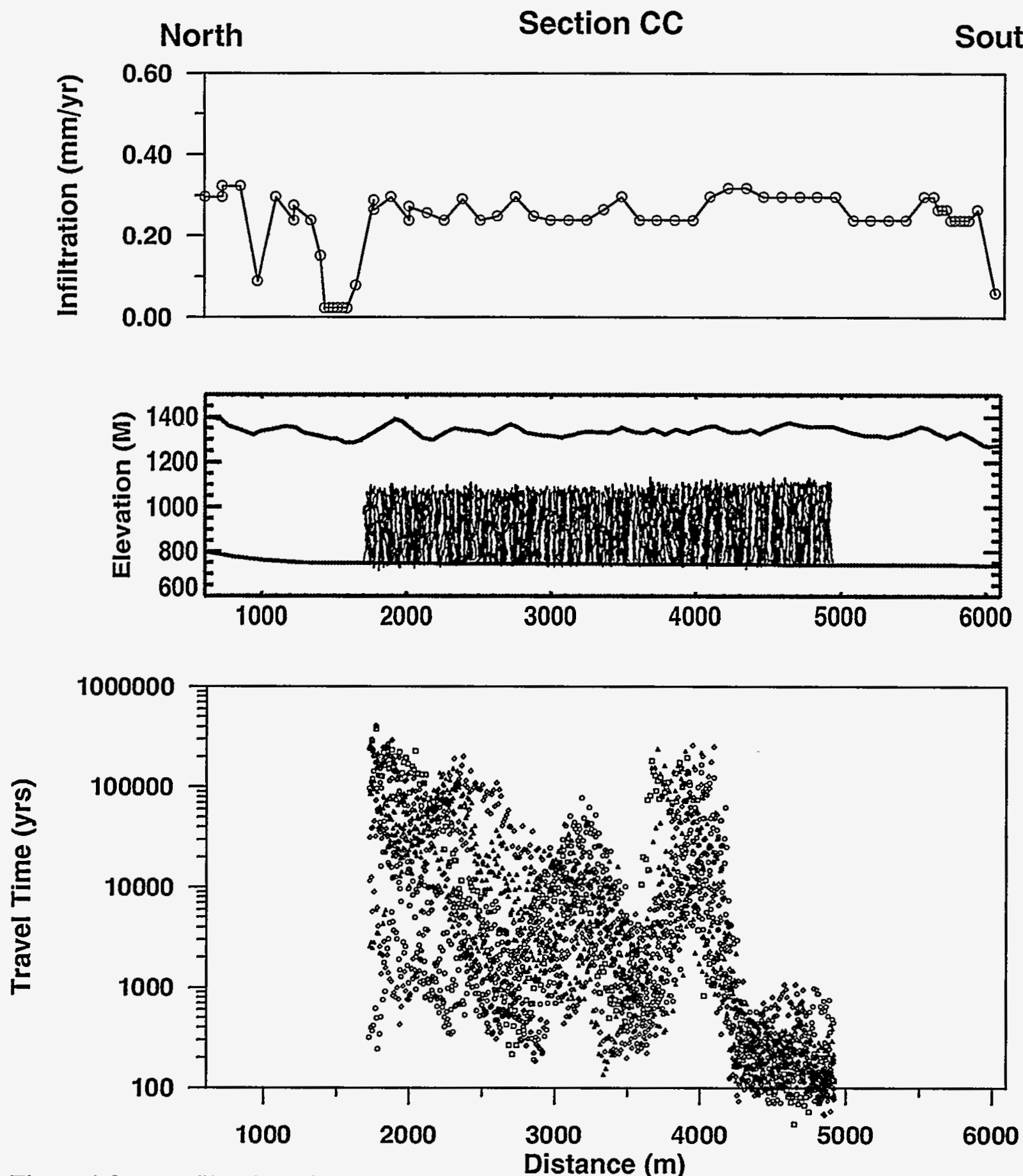

Figure 4-9c Infiltration, simulated particle paths and groundwater travel times along crosssection CC. Particle paths for a single realization of material properties are plotted in the middle figure. Portions of particle paths in which particles are advected at fracture flow velocities are plotted in blue and where particles are advected at matrix flow velocities paths are plotted in red. Travel times are plotted in the lower graph for all ten realizations. 

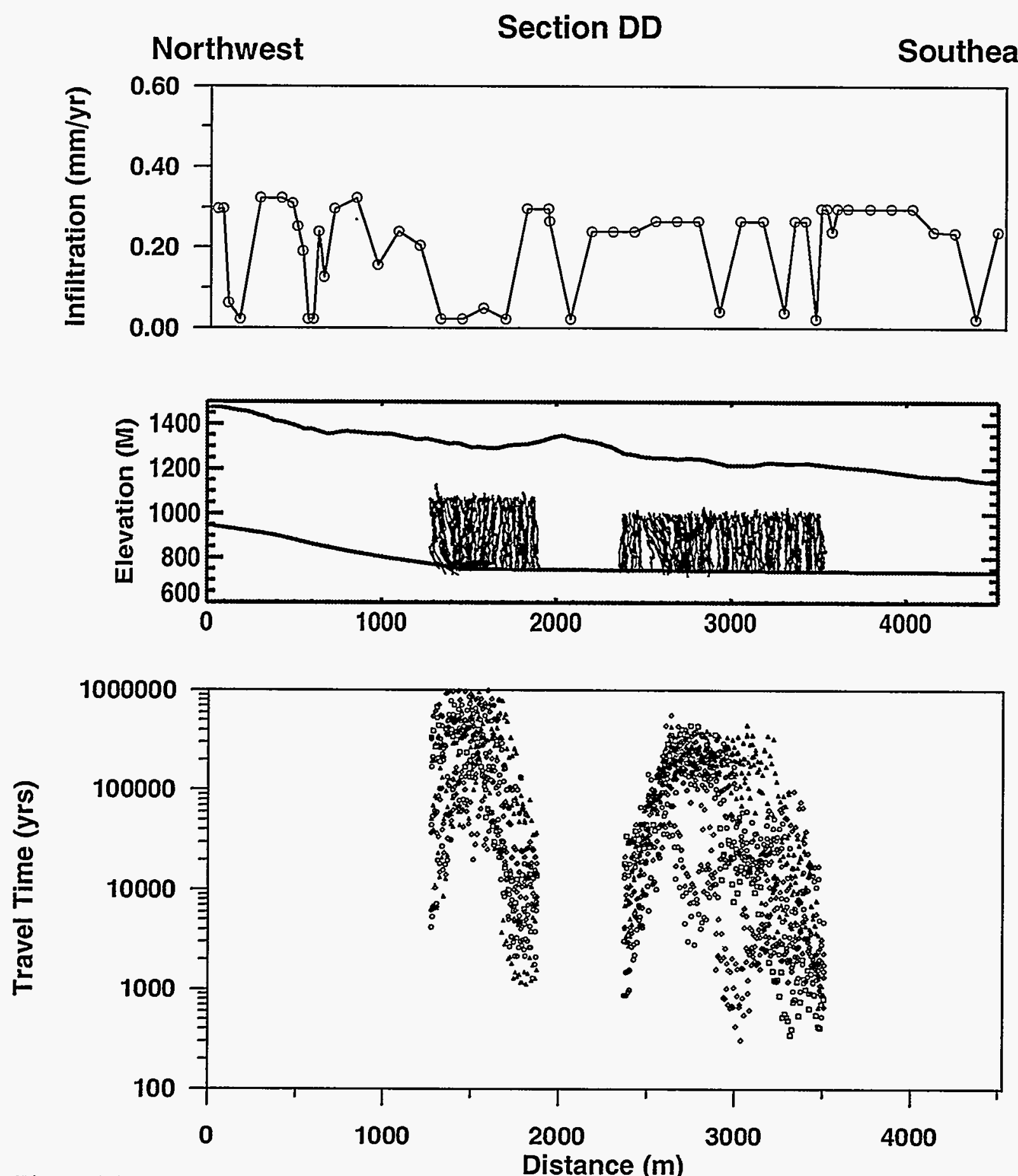

Figure 4-9d Infiltration, simulated particle paths and groundwater travel times along crosssection DD. Particle paths for a single realization of material properties are plotted in the middle figure. Portions of particle paths in which particles are advected at fracture flow velocities are plotted in blue and where particles are advected at matrix flow velocities paths are plotted in red. Travel times are plotted in the lower graph for all ten realizations. 


\subsection{Sensitivity Analysis}

Sensitivity analyses have been conducted for one geostatistical realization of one crosssection for the purpose of 1) evaluating the influence of hydrological parameters which have some uncertainty on flow simulations (infiltration, fracture-matrix connectivity, fracture frequency, fracture residual saturation, zeolitic hydrologic properties, and matrix air entry pressure or van Genuchten $\alpha$ ); 2) evaluating the modeling techniques and assumptions by varying the grid of the system; 3) examining the conceptual models of flow by altering the numerical implementation of the conceptual model (dual permeability model (DKM) and the equivalent continuum model (ECM)); and 4) examining the effects of a transient flow pulse.

Explanations for how individual analyses were performed are given in each section below. All analyses, except where noted, are on cross-section AA, realization 1. Boundary conditions, except where noted, are the same as described in Section 4.1. Also, except where noted, the dualpermeability model is used. Particles are tracked without dispersion (see Section 4.4). In most cases, the results are presented in two manners. Matrix saturations are evaluated by comparing the simulated saturation from two columns of elements to those measured from core from SD-9. Fracture saturations are not shown, but in most cases are directly correlated to matrix saturations (see Section 4.3.2). Velocity vectors are also not shown in most cases, but are evaluated through interpretations of the particle travel-time data.

\subsubsection{Infiltration Flux}

Shallow infiltration into the bedrock at Yucca Mountain is still under study. Preliminary estimates of infiltration through the different hydrostratigraphic units, regardless of locations were determined as a first iteration (Flint and Flint, 1994). More recent estimates are based on a multiple regression using precipitation, physiographic location (channel terrace, footslope, sideslope, ridge) and thickness of alluvium (greater or less than $3 \mathrm{~m}$ ) as the variables (Hudson and

Flint ${ }^{26}$ ). Based on this regression, which has a low $\mathrm{R}^{2}$ of 0.27 , average infiltration over the study area is $24 \mathrm{~mm} / \mathrm{yr}$. Over the extent of cross-section A-A the average infiltration rate is $19 \mathrm{~mm} / \mathrm{yr}$. The infiltration rates used in these analyses were taken from Hudson and Flint ${ }^{27}$ while the report was still in review and the published version may report infiltration rates somewhat different from those used in these analyses. This average rate of infiltration is much higher than has been estimated previously, thus sensitivity studies are conducted to see how a change in infiltration rate affects the saturation profiles, fluid-velocity distributions, and particle travel times through the cross-section. This section presents the work done to come to the decision to use infiltration fluxes two orders of magnitude lower than those reported in Hudson and Flint ${ }^{28}$ (Section 4.1). Saturations are compared with the original estimation of the infiltration (mean $=19 \mathrm{~mm} / \mathrm{yr}$ ), an order of magnitude lower infiltration (mean $=1.9 \mathrm{~mm} / \mathrm{yr}$ ) and two orders of magnitude lower infiltration (mean $=0.19 \mathrm{~mm} / \mathrm{yr}$ ). The base case is, therefore, a mean infiltration of $0.19 \mathrm{~mm} / \mathrm{yr}$ with the higher two infiltration rates used in the sensitivity studies.

26. see footnote 2 , page 9 .

27. Ibid.

28. Ibid. 
Matrix saturations are clearly significantly higher in the cases with the higher infiltration rates (Figure 4-10). The matrix in the units stratigraphically below the PTn are almost completely saturated in the case where the mean saturation is $19 \mathrm{~mm} / \mathrm{yr}$ and greater than $90 \%$ saturated where the mean infiltration is $1.9 \mathrm{~mm} / \mathrm{yr}$. The best match (of the three mean infiltration rates considered) to the measured saturations from core from SD- 9 is for the base case (mean infiltration $=0.19$ $\mathrm{mm} / \mathrm{yr}$ ) (Figure 4-10). Fracture saturations are also significantly increased with the higher infiltration fluxes (not shown).

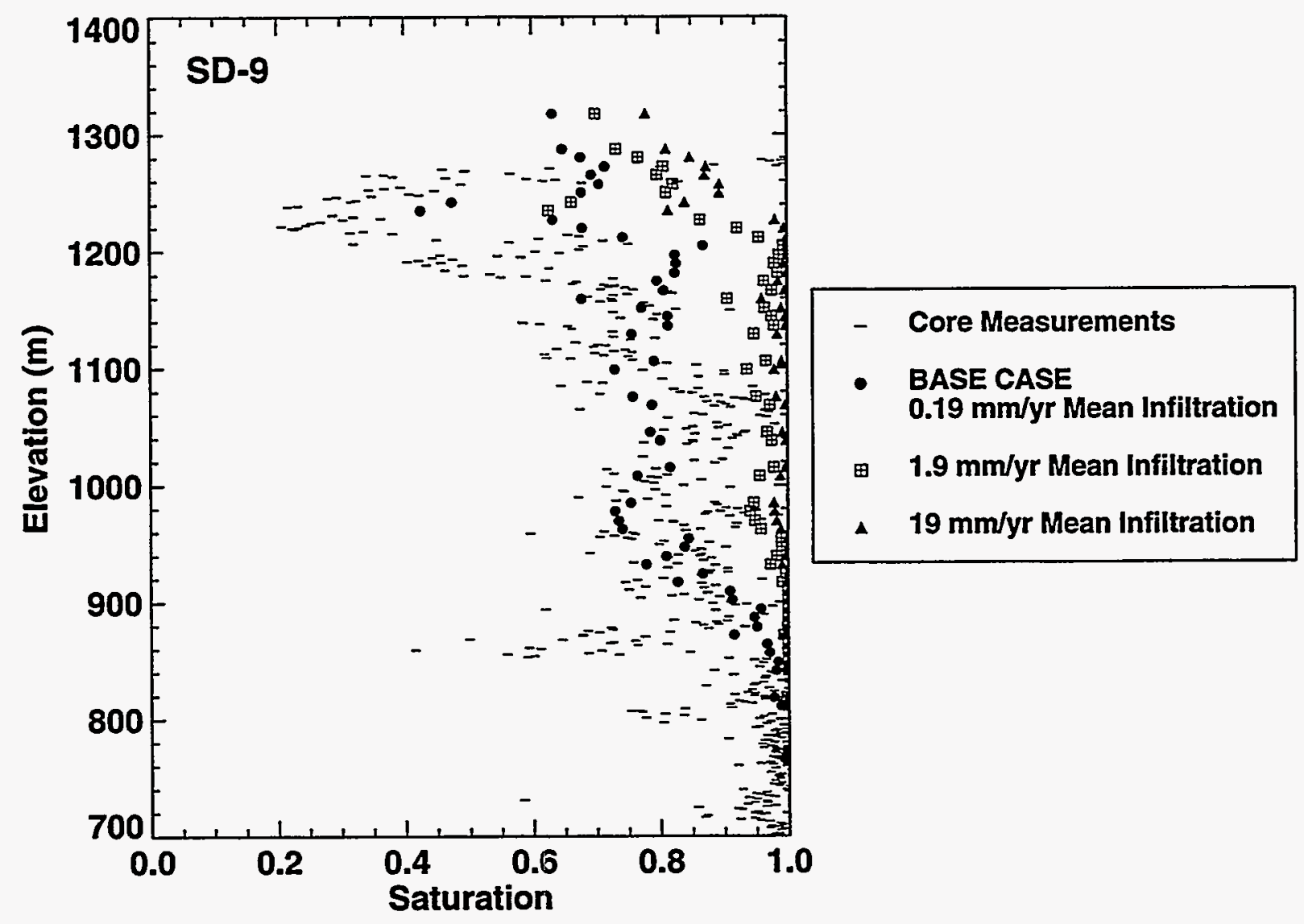

Figure 4-10 Comparison of simulated saturations using different spatially variable infiltration rates to measured core saturations from SD-9.

The differences in infiltration rate also have a strong effect on groundwater flow velocities and, therefore, particle travel times. Particle travel times decrease by more than an order of magnitude when the mean infiltration is increased from 0.19 to $1.9 \mathrm{~mm} / \mathrm{year}$ and again by approximately an order of magnitude when the mean infiltration is increased from 1.9 to $19 \mathrm{~mm} /$ year (Figure 4-11). In summary, infiltration rate clearly has a strong control on both saturations and flow velocities of the system. 


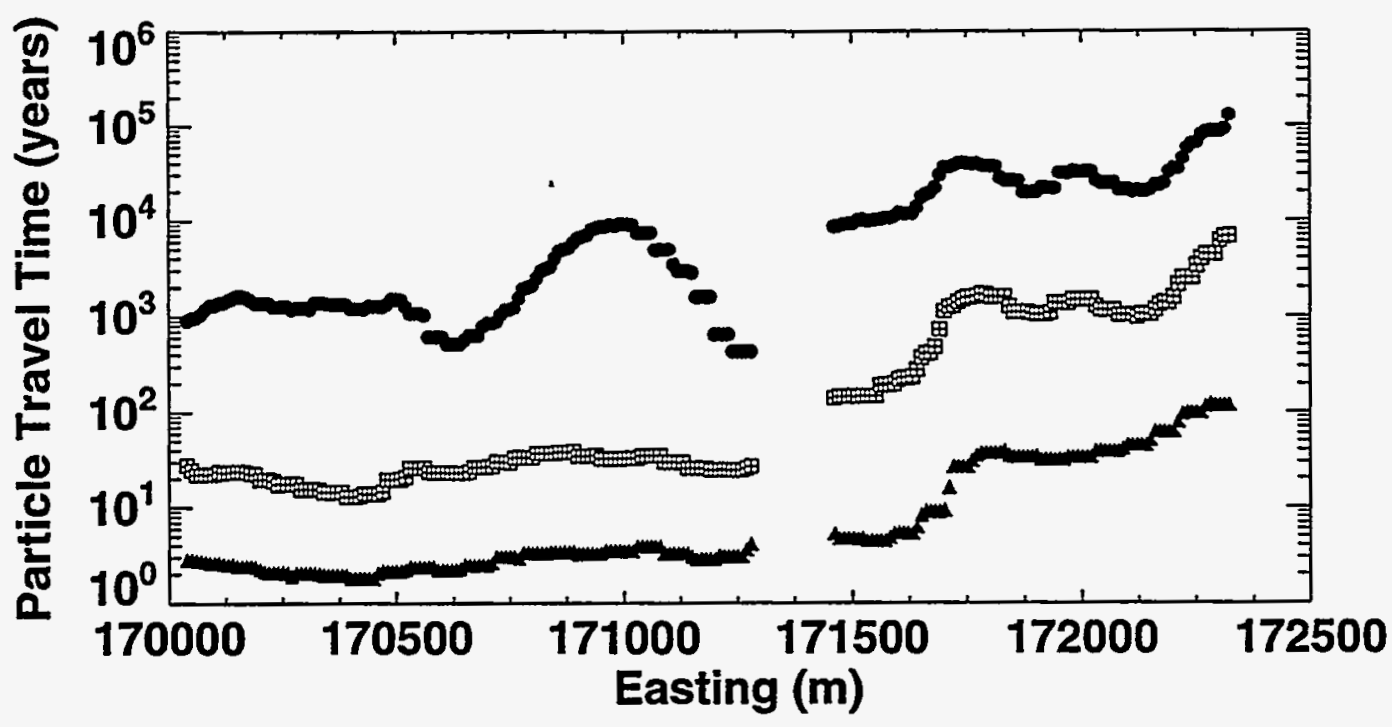

- base CASE $0.19 \mathrm{~mm} / \mathrm{yr}$ Mean Infiltration

ד $1.9 \mathrm{~mm} / \mathrm{yr}$ Mean Infiltration

$1 \quad 19 \mathrm{~mm} / \mathrm{yr}$ Mean Infiltration

Figure 4-11 Particle travel times along cross-section AA for flow simulations using different spatially variable infiltration rates.

\subsubsection{Fracture-Matrix Connectivity Scaling}

Fracture-matrix connectivity is another parameter for which there is uncertainty. For the GWTT-95 calculations using the DKM, the fracture-matrix connectivity is set to be three orders of magnitude less than full geometric connection. This reduction is justified for two reasons. First, water is not flowing through every fracture at Yucca Mountain. Second, on the surface of a fracture with water flowing through it, due to fingering and fracture coating, it is unlikely that the entire surface will be wetted (Glass et al., ${ }^{29}$ Chekuri et al., 1994). Clearly, there is a high degree of uncertainty in what proportion of the fracture domain will be wetted. Of interest in reducing the fracture-matrix connectivity is whether in-situ matrix saturations could be matched in a manner different than reducing the infiltration rate from its original estimation (Section 4.5.1). The influence of fracture-matrix connectivity on the flow velocities and therefore particle travel times is also of interest. The sensitivity of this fracture-matrix connectivity is tested with two additional calculations: one reducing the full connection by 4 orders of magnitude, and a second

29. Glass, R. J., M. J. Nicholl, and V. C. Tidwell. in press. Challenging and Improving Conceptual Models for Isothermal Flow in Unsaturated, Fractured Rock through Exploration of Small-Scale Processes. SAND95-1824. Albuquerque, NM: Sandia National Laboratories. 
using a 5-order-of-magnitude reduction. Each fracture matrix-connectivity (reduced by 3, 4, and 5 orders of magnitude) is run at two different infiltration rates (mean infiltration $=0.19$ and 1.9 $\mathrm{mm} / \mathrm{yr}$ ). The base case is the simulation using a mean infiltration of $0.19 \mathrm{~mm} / \mathrm{yr}$ and a 3 order of magnitude fracture-matrix reduction.

In-situ saturations measured from core samples from SD-9 can be matched by inversely adjusting fracture-matrix connectivity and infiltration flux. It can be seen that with both a 3-orderof-magnitude fracture matrix connectivity reduction and a mean infiltration rate of $0.19 \mathrm{~mm} / \mathrm{yr}$ and a 5-order-of-magnitude fracture matrix connectivity reduction and a mean infiltration rate of $1.9 \mathrm{~mm} / \mathrm{yr}$ the in-situ saturations are equally well matched (Figure 4-12). These results are significant in that they demonstrate that using matrix saturations as a calibration measure can lead to non-unique solutions. As expected, matrix saturations decrease with a decrease in fracturematrix connectivity due to less interaction between the fracture and matrix domain (Figure 4-13). Remember that infiltration along the upper boundary is introduced into the fracture domain. Thus, with this decrease in fracture-matrix interactions, the water is more likely to stay in the fracture domain.

Reduction of fracture-matrix connectivity has a stronger effect on the particle travel times at lower infiltration rates (Figure 4-14). At both mean infiltration rates of 0.19 and $1.9 \mathrm{~mm} / \mathrm{yr}$ reducing the fracture matrix-connectivity leads to a decrease in particle travel times; the reductions are more dramatic at the lower infiltration rate. Note that in the eastern portion of the cross-section the infiltration rates are lower, yielding larger differences in particle travel times in this section for the case with a mean infiltration rate of $1.9 \mathrm{~mm} / \mathrm{yr}$. As stated above, using saturations as a calibration measure leads to non-unique solutions, and in the cases demonstrating this (3 order of magnitude fracture-matrix connectivity and $0.19 \mathrm{~mm} / \mathrm{yr}$ mean infiltration rate and 5 order of magnitude fracture-matrix connectivity and $1.9 \mathrm{~mm} / \mathrm{yr}$ mean infiltration rate) the resulting simulations lead to two order of magnitude different particle travel times (Figure 415).

\subsubsection{Fracture Frequency}

Interpretation of fracture frequency data from core logs is somewhat subjective. In Section 3.2.2.1 a correction for the increased likelihood of a vertical borehole intersecting more flat lying fractures is described in which the dip of the fracture is taken into account. For the GWTT-95 data a $10^{\circ}$ dip interval is used (see equation 3-1). However, due to uncertainty in the dip measurements, it is possible that using a $30^{\circ}$ interval would be more representative of the accuracy of the fracture dip data. For this reason, fracture frequency data are re-corrected using the same methods described in Section 3.2.2.1 only using $30^{\circ}$ dip intervals. Equation 3-1 therefore becomes:

$$
\begin{aligned}
& \text { Corrected Fracture } \#=\frac{\# \text { of Fractures with dips between } 0 \text { and } 30^{\circ}}{\cos 15}+ \\
& \frac{\# \text { of Fractures with dips between } 30 \text { and } 60^{\circ}}{\cos 45}+\frac{\# \text { of Fractures with dips between } 60 \text { and } 90^{\circ}}{\cos 75}
\end{aligned}
$$




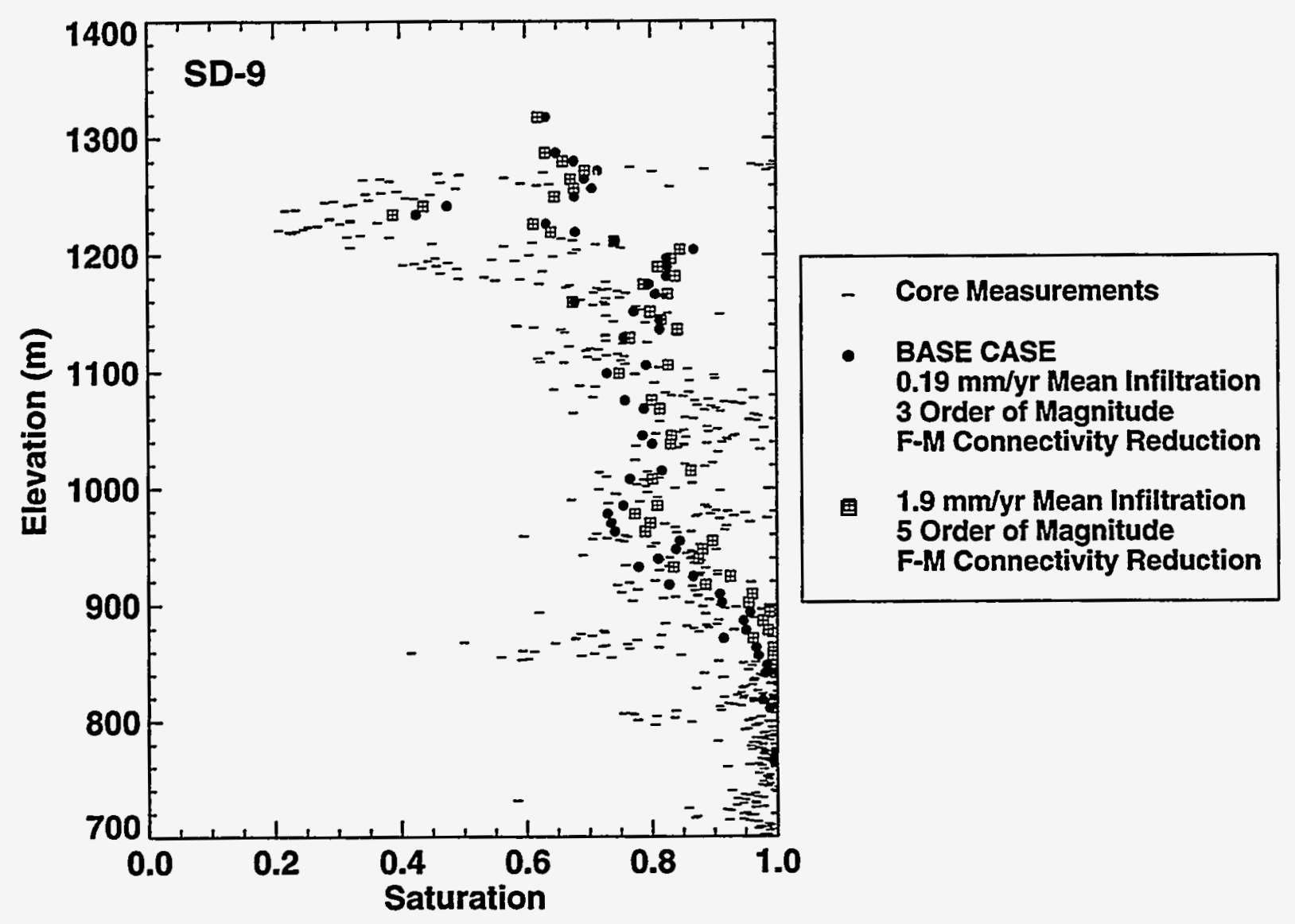

Figure 4-12 Comparison of simulated saturations to measured core saturations from SD-9 using a 3 order of magnitude and 5 order of magnitude reduction to full fracturematrix connection and a mean infiltration rate of 0.19 and $1.9 \mathrm{~mm} / \mathrm{yrm}$ respectively. Note that both simulated saturation profiles match the in-situ saturations comparably well.

Corrected fracture frequencies are re-calculated and new mean fracture frequencies are calculated for each unit (Table 4-2). A new geostatistical fracture frequency field is generated using these re-corrected fracture frequencies for the means and conditioning data. Examination of re-corrected NRG-6 borehole fracture frequency data show that the resultant fracture frequencies are less than the data used in the GWTT-95 analyses, on average, by a factor of approximately 1.6 (Figure 4-16).

Calculated fracture porosity is also dependent on fracture frequency (see Section 3.2.2.2). Therefore, a new regression relationship between matrix and fracture porosity was constructed and used in this sensitivity analyses (Figure 4-17).

While not directly used in the flow model, fracture frequency is used to calculated fracture permeability, a model input parameter (equation 3-3). Also, because fracture frequency is used to generate the relationship between matrix and fracture porosity, changing the fracture frequency 
(a)

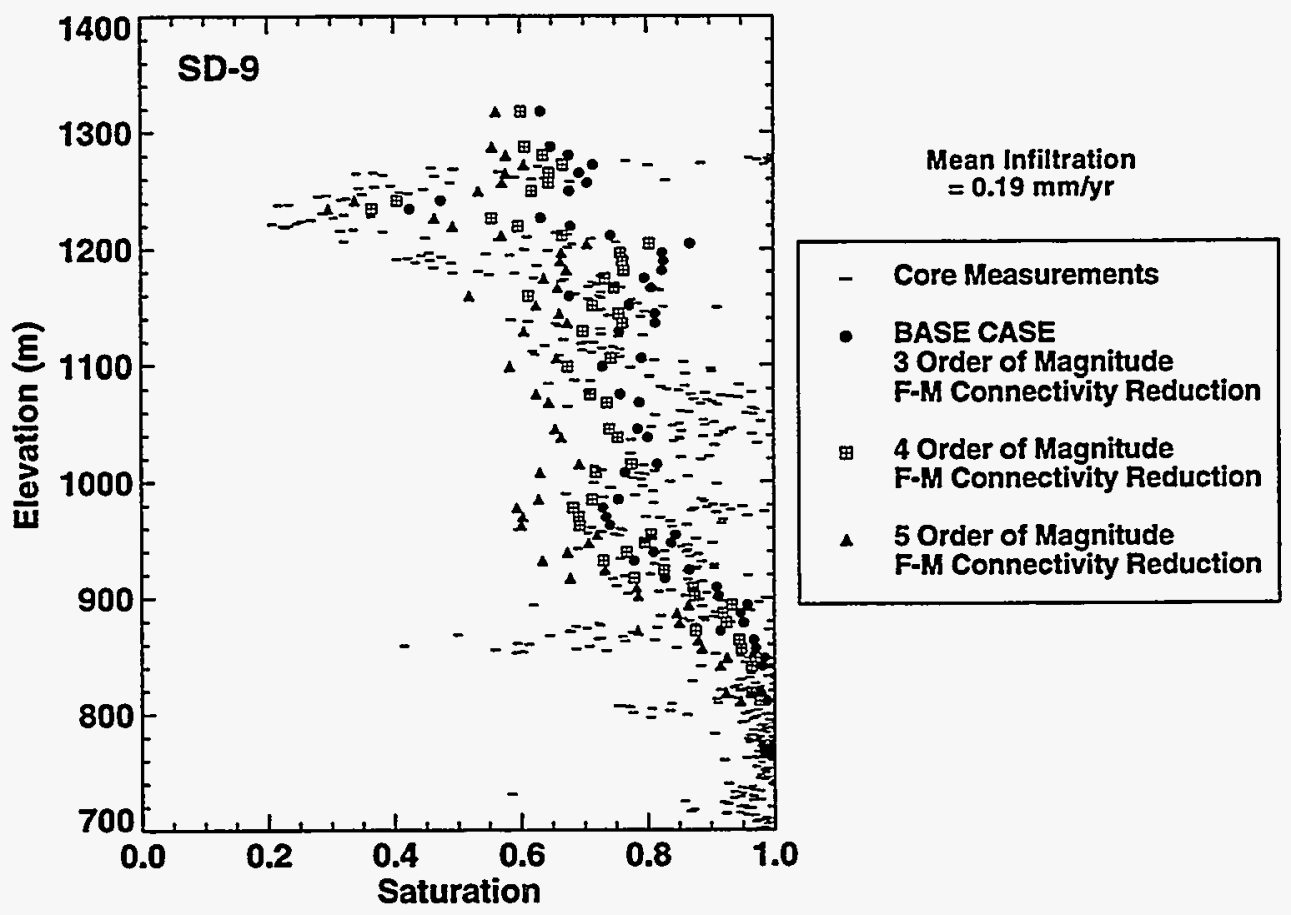

(b)

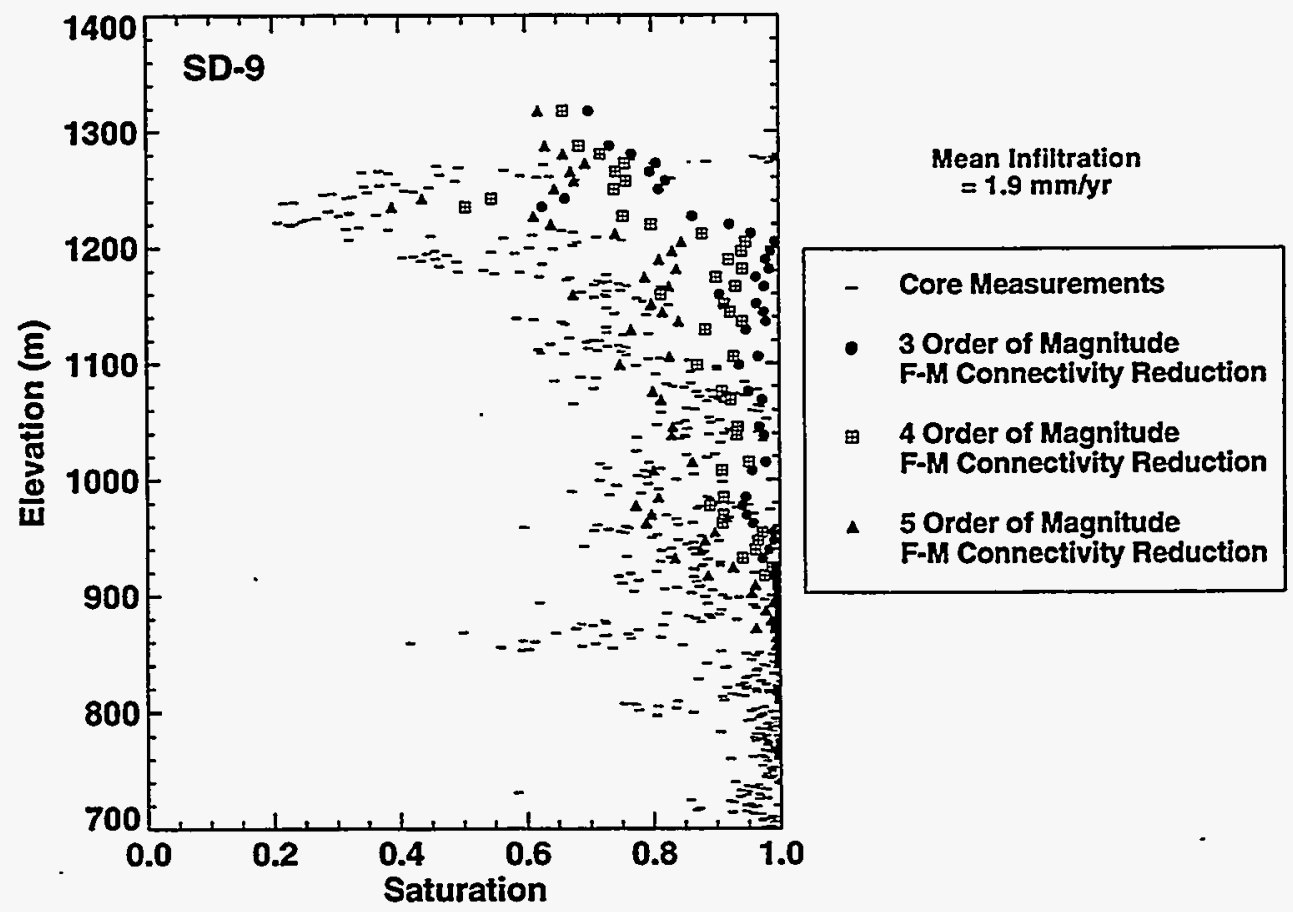

Figure 4-13 Comparison of simulated saturations to measured core saturations from SD-9 using different reductions to full fracture-matrix connection for a mean infiltration rate of (a) $0.19 \mathrm{~mm} / \mathrm{yr}$ and (b) $1.9 \mathrm{~mm} / \mathrm{yr}$. Note the decrease in matrix saturations with an increased reduction of fracture-matrix connectivity at both infiltration rates. 

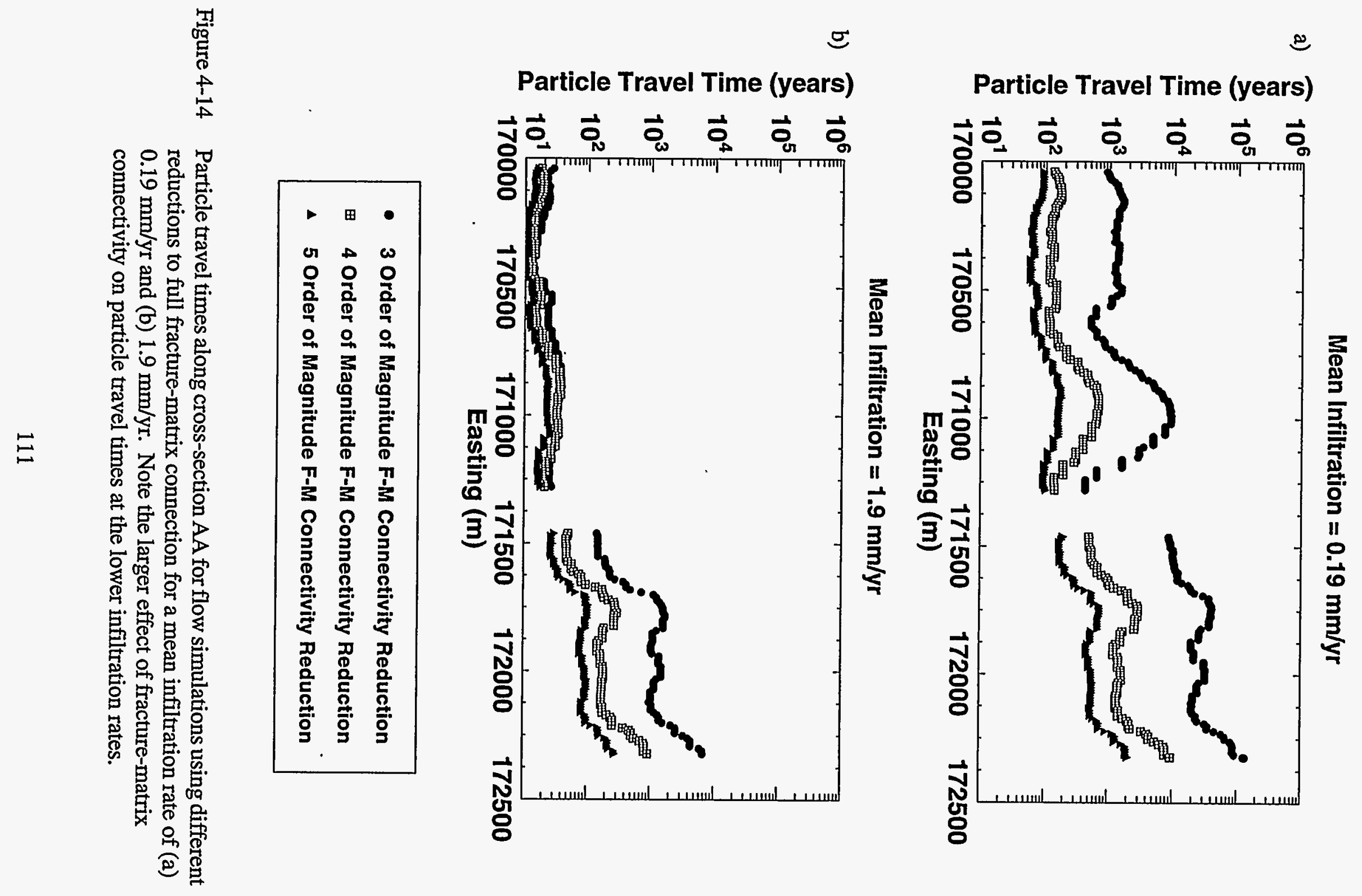


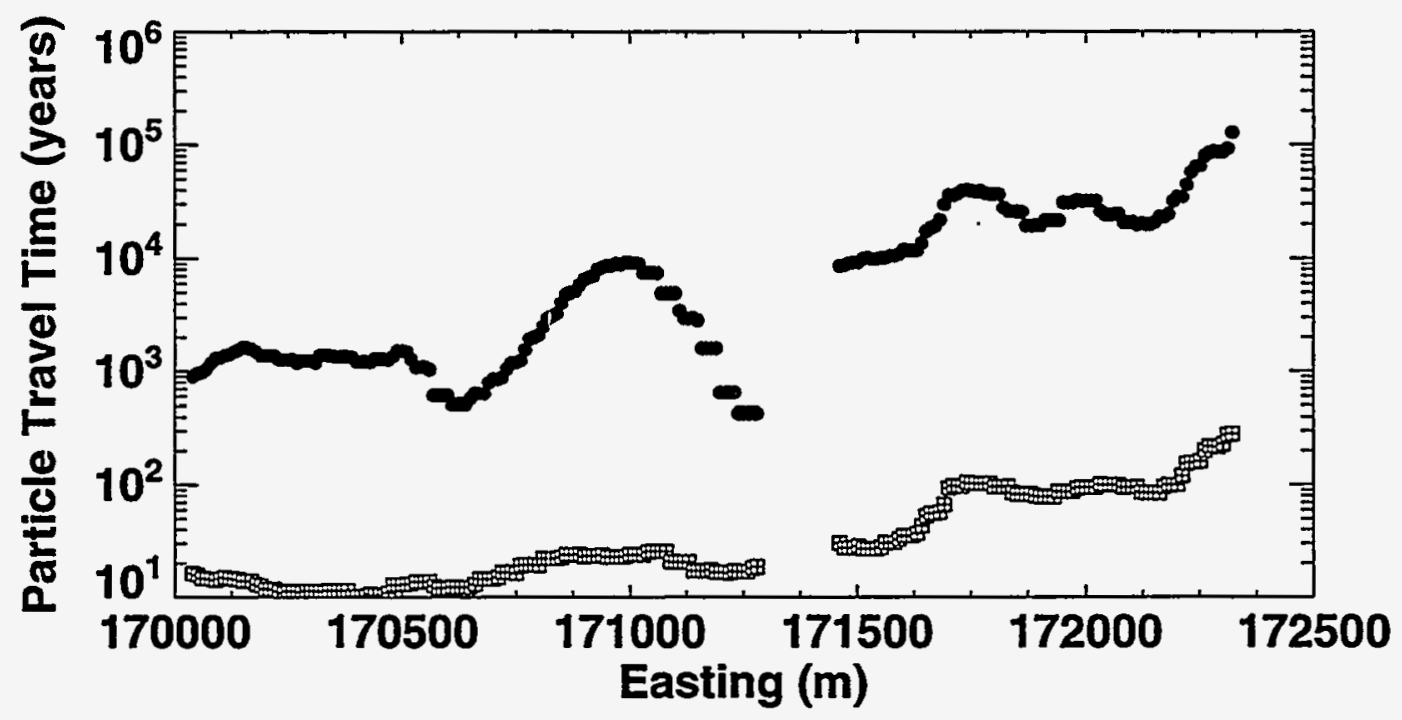

- base case

$0.19 \mathrm{~mm} / \mathrm{yr}$ Mean Infiltration

3 Order of Magnitude F-M Connectivity Reduction

\# $1.9 \mathrm{~mm} / \mathrm{yr}$ Mean Infiltration

5 Order of Magnitude F-M Connectivity Reduction

Figure 4-15 Particle travel times along cross-section AA for flow simulations using a 3 order of magnitude and 5 order of magnitude reduction to full fracture-matrix connection and a mean infiltration rate of 0.19 and $1.9 \mathrm{~mm} / \mathrm{yr}$, respectively. Note the almost two-order-of-magnitude difference in particle travel times despite the similar calculated saturations for the two simulations.

will also change fracture porosity, another input parameter. With the new regression, fracture porosity will be lower than that of the base case for the welded units, but similar to the base case for the nonwelded units. Since fracture frequency and porosity are used to calculate fracture aperture (equation 3-2), this parameter will also differ from the base case, with an increase for the nonwelded units. The effect of changing the fracture frequency on the fracture aperture is more difficult to assess for the welded units since the relative decrease in fracture frequency and fracture porosity vary from element to element. However, with just a small increase in fracture aperture the fracture permeability increases because of the aperture cubed term in equation 3-3. A decrease in fracture frequency also causes a decrease in the fracture-matrix connectivity (Section 2.3). An increase in the fracture permeability and the decrease in fracture-matrix connectivity should result in increased flow velocities. The relative influence of the fracture-matrix connectivity is evaluated by comparing the effects of changing the connectivity (Section 4.5.2) to the results of changing the fracture frequency. All simulations use a mean infiltration rate of 0.19 $\mathrm{mm} / \mathrm{yr}$. 

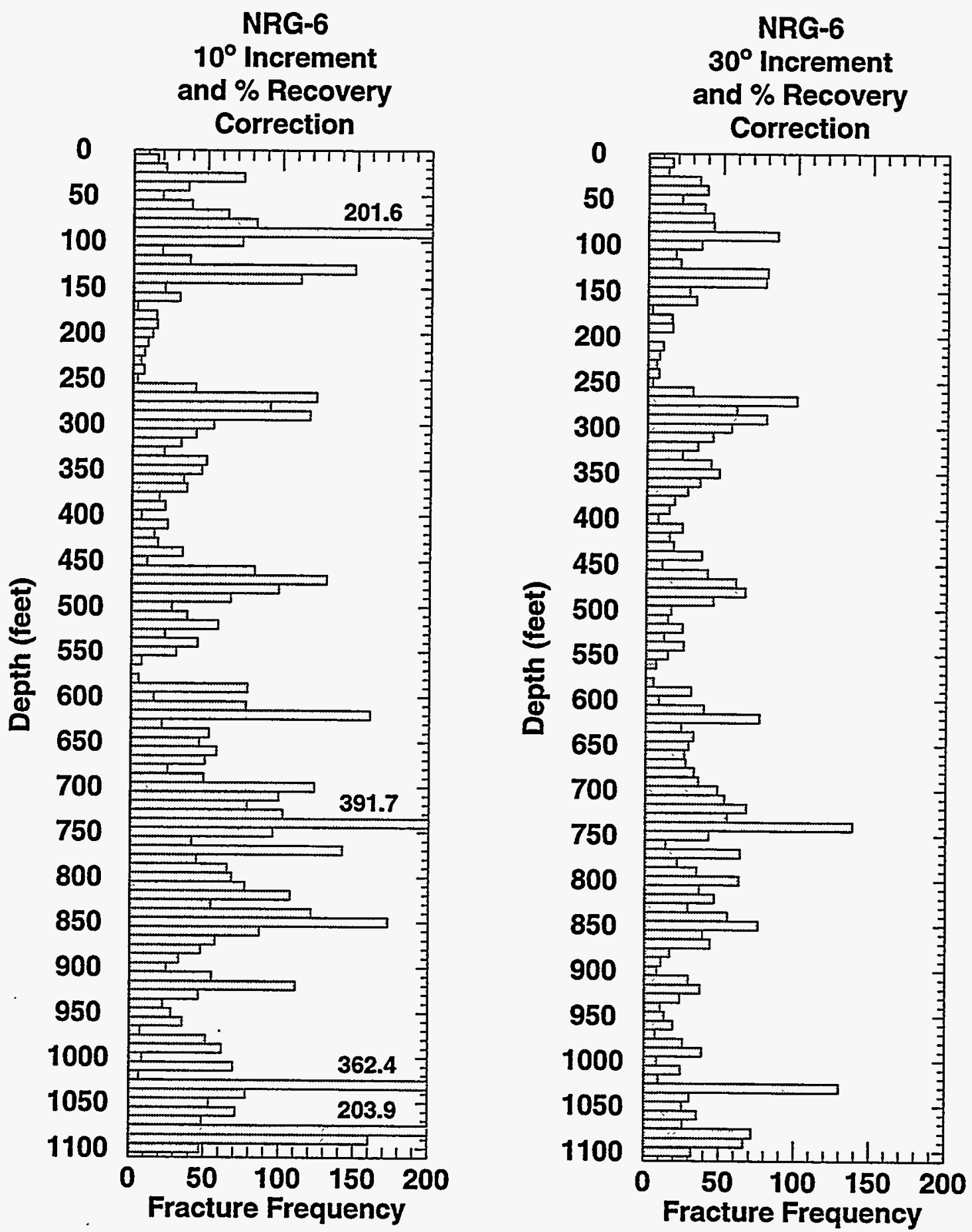

Figure 4-16 Corrected fracture frequency data from borehole NRG-6 using $10^{\circ}$ and $30^{\circ}$ increments of dip measurements for correction (see equation 3-1). 


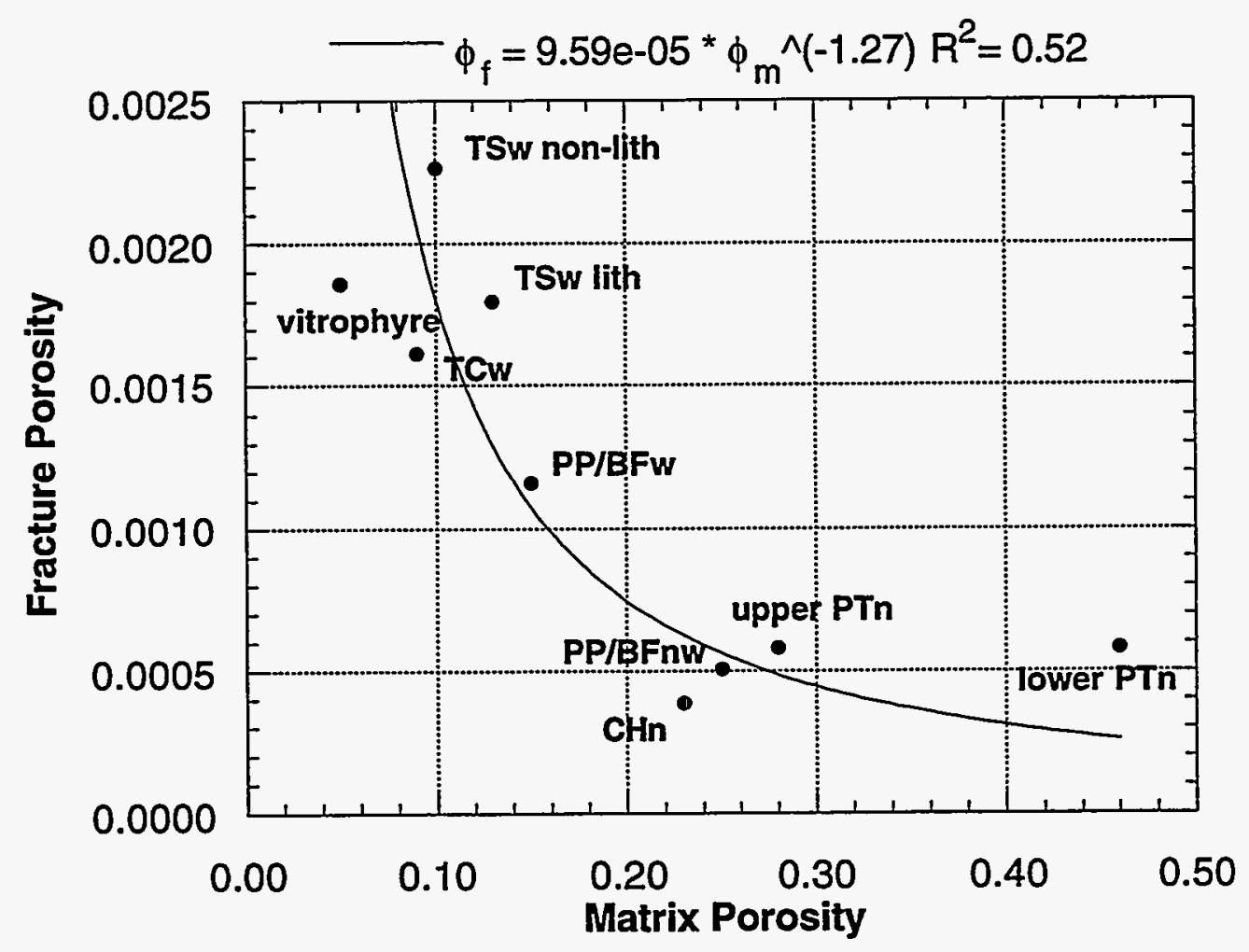

Figure 4-17 Regression relationship between.matrix and fracture porosity using fracture frequency data using $30^{\circ}$ increments of dip measurements for correction used for GWTT-95 fracture frequency sensitivity study. This regression relationship is adjusted to preserve the fracture aperture distribution, as described in the Section 3.2.2.2.

Reduction of fracture frequency decreases the simulated matrix saturation slightly more than the results of decreasing the fracture-matrix connectivity by 5 orders of magnitude (Figure 418). This decrease in matrix saturation is expected with a decrease in fracture-matrix connectivity and in increase in fracture permeability which will both decrease the likelihood of matrix imbibition. Fracture saturations increase as would be expected by decreasing the fracture-matrix connectivity (not shown). Particle travel times decrease slightly more than when the fracturematrix connectivity is directly reduced (Figure 4-19). This observation can be explained by the higher fracture saturations which would result in a higher hydraulic conductivity. .

\subsubsection{Fracture Residual Saturation}

Fracture residual saturation is a parameter for which there are not any actual measurements. It is often assumed to be 0 . Unfortunately, setting the fracture residual saturation to 0 causes numerical complications when running TOUGH2. A fracture residual saturation of 0.0001 was thought to be sufficiently low and was used for the GWTT-95 simulations. As a 


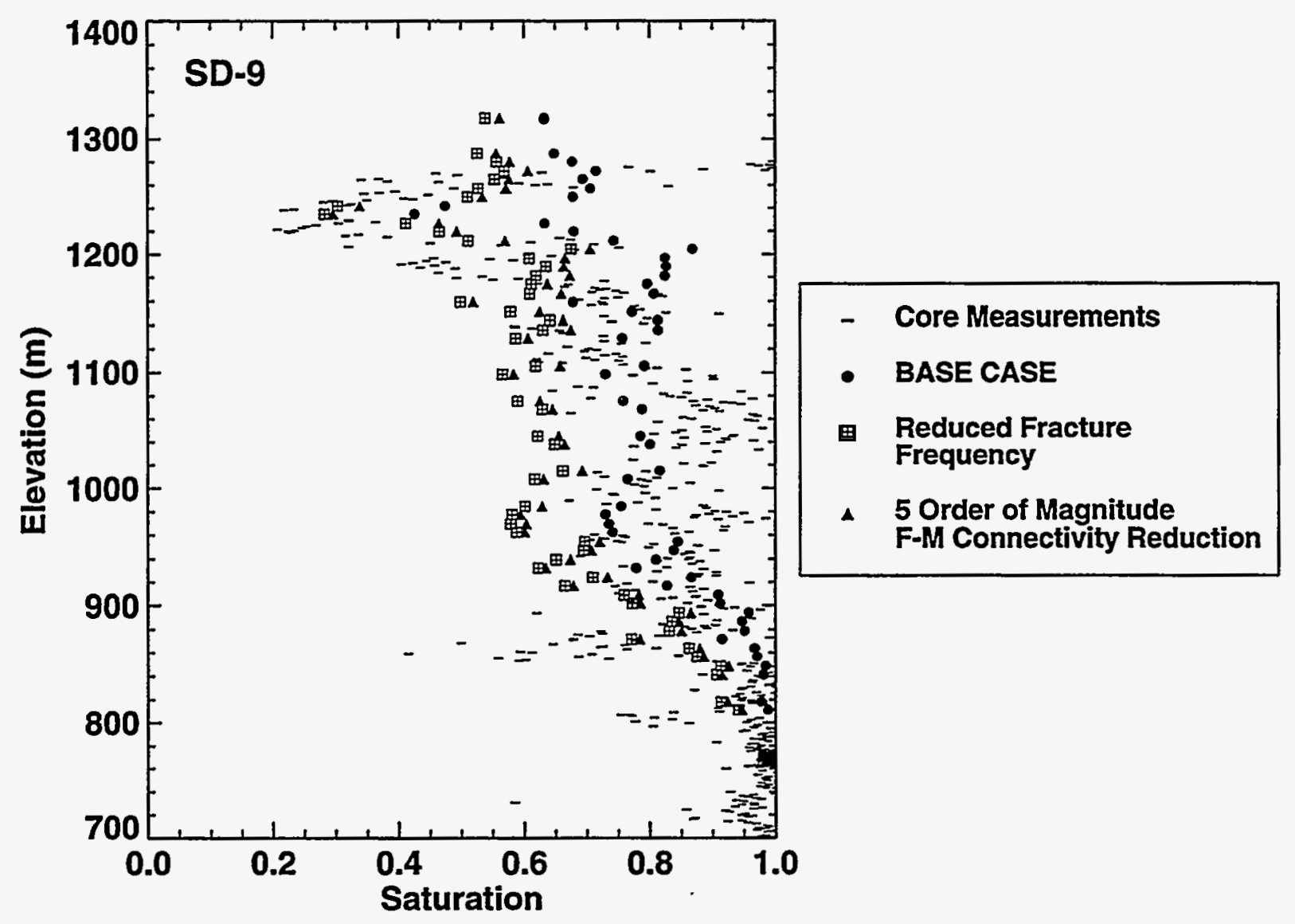

Figure 4-18 Comparison of simulated saturations to measured core saturations from SD-9 for a reduced fracture frequency. Saturations values for a reduced fracturematrix connectivity (see Section 4.5.2) are included for comparison.

Table 4-2: Mean fracture frequency and the probability of hitting a 10 foot interval without a fracture for each hydrogeological unit used for sensitivity study.

\begin{tabular}{|l|c|c|}
\hline Hydrostratigraphic Unit & $\begin{array}{c}\text { Mean Fracture } \\
\text { Frequency (1/m) }\end{array}$ & $\begin{array}{c}\text { Probability of Hitting a 10 foot } \\
\text { Interval without a Fracture }\end{array}$ \\
\hline \hline TCw & 9.00 & 0.0 \\
\hline PTn & 2.68 & 0.22 \\
\hline TSw nonlithophysal & 11.17 & 0.015 \\
\hline TSw lithophysal & 9.00 & 0.086 \\
\hline CHn & 1.95 & 0.46 \\
\hline vitrophyres & 8.28 & 0.071 \\
\hline
\end{tabular}




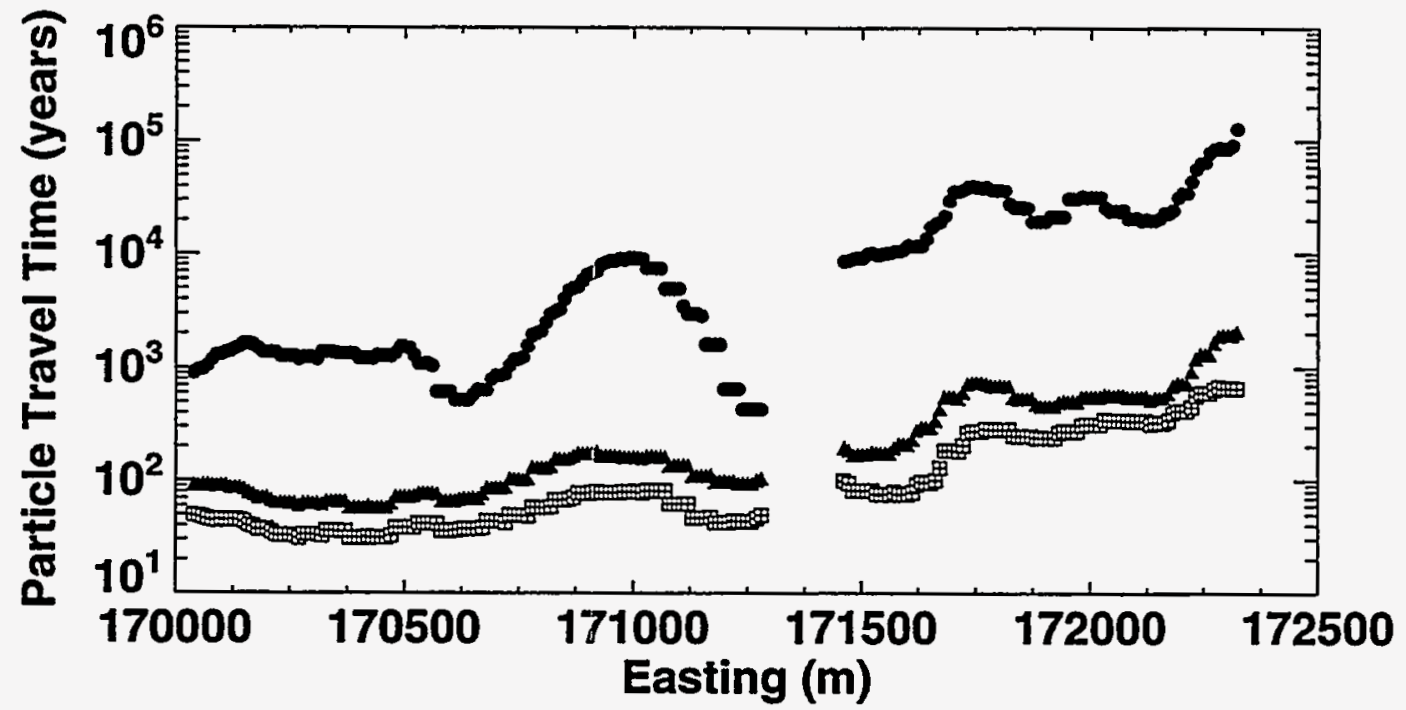

\section{- base CASE \\ 田 Reduced Fracture Frequency \\ $\Delta \quad 5$ Order of Magnitude F-M Connectivity Reduction}

Figure 4-19 Particle travel times along cross-section A-A for flow simulations using a reduced fracture frequency. Travel times for a reduced fracture-matrix connectivity (see Section 4.5.2) are included for comparison.

sensitivity study, it was decided to vary the residual saturation to see how much of an influence it has on the saturation and flow fields in the GWTT-95 studies. An alternative fracture residual saturation of 0.00001 is used in flow simulations.

With the decrease in fracture residual saturation, the fractures are allowed to dry out more. In the washes the fractures clearly dry to the maximum extent of fracture residual saturations of 0.0001 and 0.00001 . Fracture saturation profiles for simulations run with residual saturations of 0.0001 and 0.00001 are quite similar except in the wash areas and just to the west along the Ghost Dance fault. In these locations the saturations decrease to below 0.0001 when the residual saturation is set to 0.00001 . When the fracture residual saturation is set to 0.00001 the fractures in the washes eontinue to dry, but not to saturations as low as 0.00001 . 
The decrease in fracture saturations does not effect the particle travel times significantly (Figure 4-20). These results are not surprising because, even if the fracture saturations are lower when the fracture residual saturation is set to 0.0001 , remember that the calculated pore velocity assumes that the water held as residual is immobile (see equation 4-9), thus, due to the $\left(\theta-\theta_{\text {resid }}\right)$ term, the pore velocities should not differ too much for the two different residual saturations.

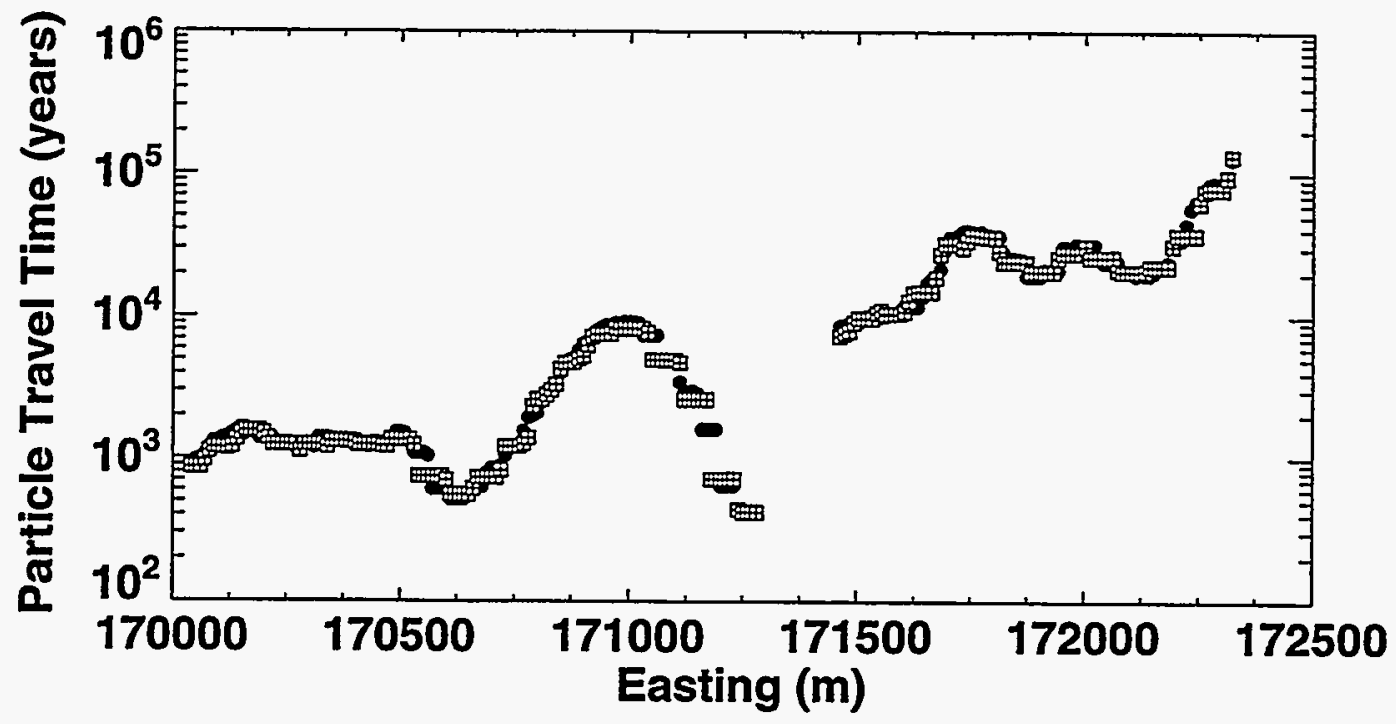

- bASE CASE

Fracture Residual Saturation $\mathbf{= 0 . 0 0 0 1}$

田 Fracture Residual Saturation $=\mathbf{0 . 0 0 0 0 1}$

Figure 4-20 Particle travel times along cross-section AA for flow simulations using different fracture residual saturations.

\subsubsection{Zeolitic Region}

Currently work is being done to delineate the regions of the Calico Hills and Prow Pass units to better determine where zeolitic alterations have occurred. As of this study, there is still uncertainty in how to demarcate these areas and the best available data were used (Section 3.1.1). The influence these zeolitic regions have on the hydrologic system is the dramatic decrease in hydraulic conductivity in these areas (Figure 3-5). However, the effect of this alteration on the flow system and the particle travel times has not been assessed. For this reason, the geostatistical 
simulation for cross-section A-A, realization 1, is re-run without delineating a zeolitic region. This change should only affect the simulated saturated hydraulic conductivities in the zeolitic regions.

Saturation profiles show a much lower saturation, relative to the base case, in the lower part of the section where the zeolitic properties have been removed (Figure 4-21). These results are not surprising; with an increased conductivity water can flow more freely and the pore spaces of the non-welded units will not fill as much (i.e. lower saturations). In addition, the fracture saturations in the zeolitic region decreased with the removal of the zeolitic region properties. This decrease is most likely due to the ability of the matrix domain to imbibe more water with the rise in hydraulic conductivity. The effect of increasing the hydraulic conductivity in the non-welded units is to increase the particle travel times, though much less than an order of magnitude (Figure 4-22). While intuitively this sounds contradictory, remember that in most cases the faster path is through the fracture domain. Thus, with the increase in matrix conductivity the fluxes in the matrix domain will increase leading to a decrease in the fluxes in the fracture domain and therefore slower particle travel times.

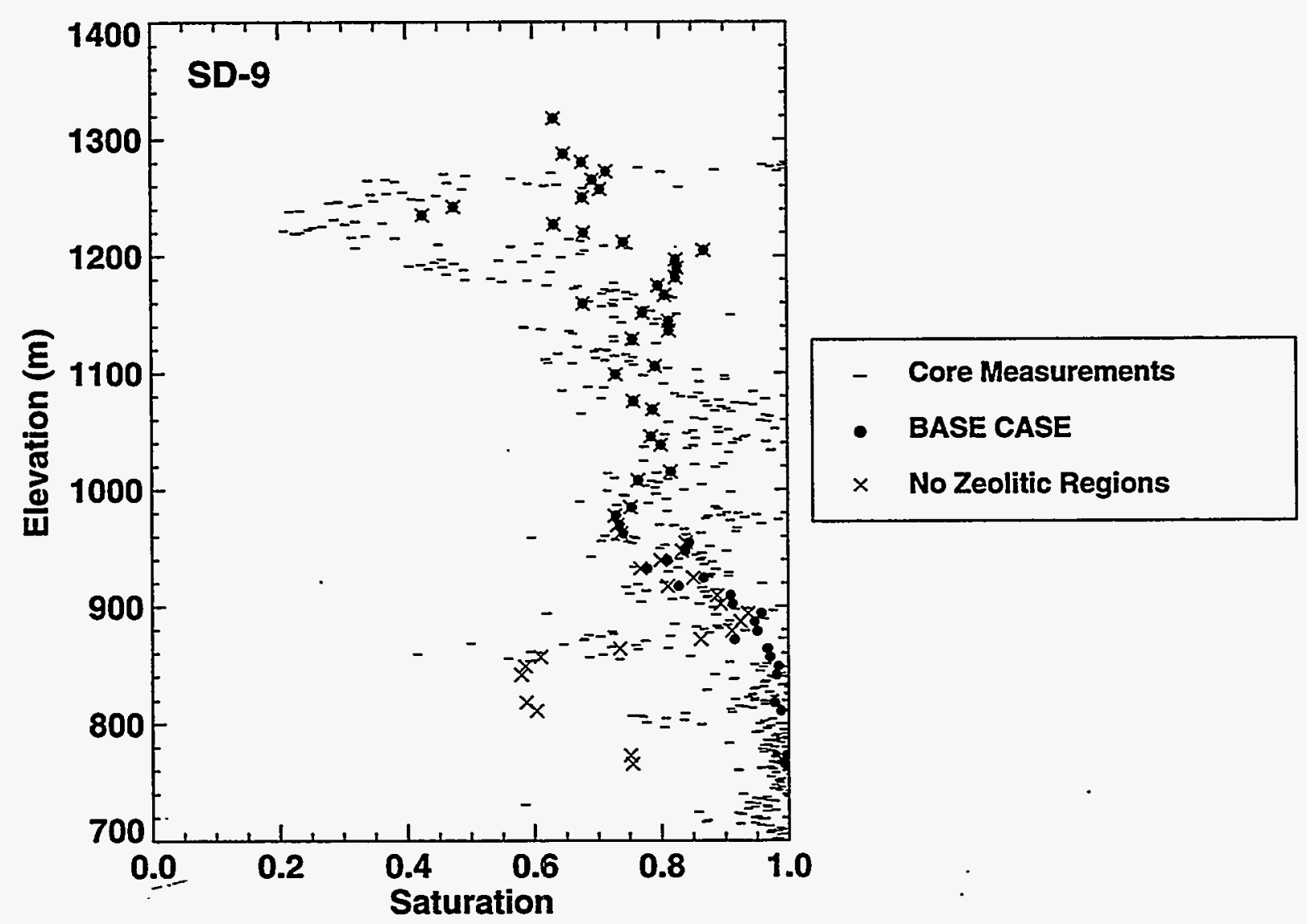

Figure 4-21 Comparison of simulated saturations to measured core saturations from SD-9 for simulations with and without the hydrologic properties of a zeolitic region. 


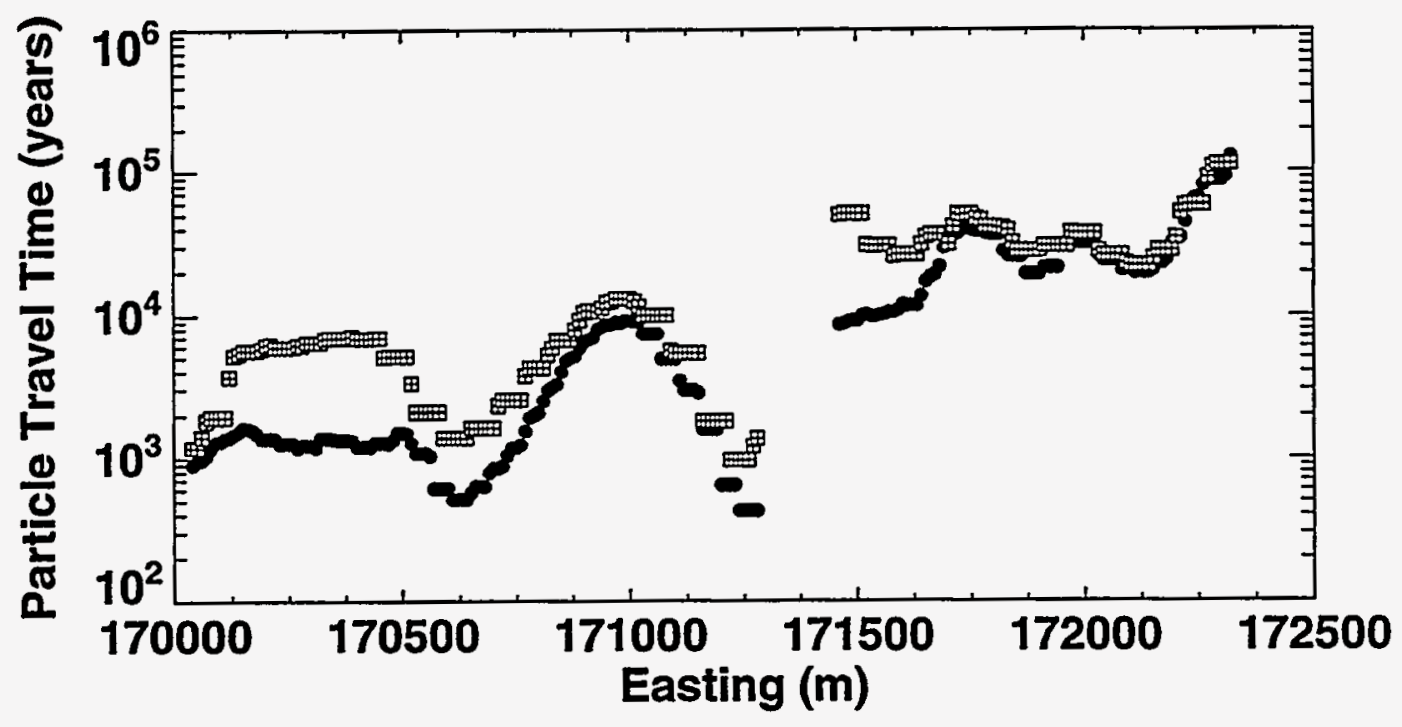

- bASE CASE

$\rightarrow$ No Zeolitic Region

Figure 4-22 Particle travel times along cross-section AA for flow simulations with and without the hydrological properties of a zeolitic region.

\subsubsection{Numerical Grid}

Modeling techniques are evaluated by refining the grid used in cross-section A-A. Only the western portion of the cross-section is refined to just east of the Ghost Dance fault. The resulting grid is refined by splitting each element in half both vertically and transversely, resulting in a grid with 3096 elements (6192 including the fracture domain for dual-permeability modeling). Geostatistically simulated properties are upscaled to the finer grid and derived properties are recalculated for each element.

In general, the matrix saturations for the two grids match very well (Figures 4-23 and 425). Simulated matrix saturations in the PTn using the finer grid better match in-situ measurements than those simulated using the coarser grid (Figure 4-25). Heterogeneity is expected to be higher in the fine grid domain, as less averaging is necessary within an element. This increased heterogeneity is manifested in the matrix saturations where there is a larger range in saturations when a finer grid is used. For example, in the TSw the matrix saturations can be seen to range from approximately 0.6 to 1 with the finer grid, but only from approximately 0.7 to 0.9 when a courser grid is used (Figure 4-23). Differences in fracture saturations are even more noticeable (Figure 4-24). The fracture domain is clearly dryer, especially under the peak, when 
the finer grid is used. Fracture saturations are also lower in the Calico Hills. Perhaps, due to the increased heterogeneity in the domain with the finer grid, more imbibition is possible, thus drying out the fractures.

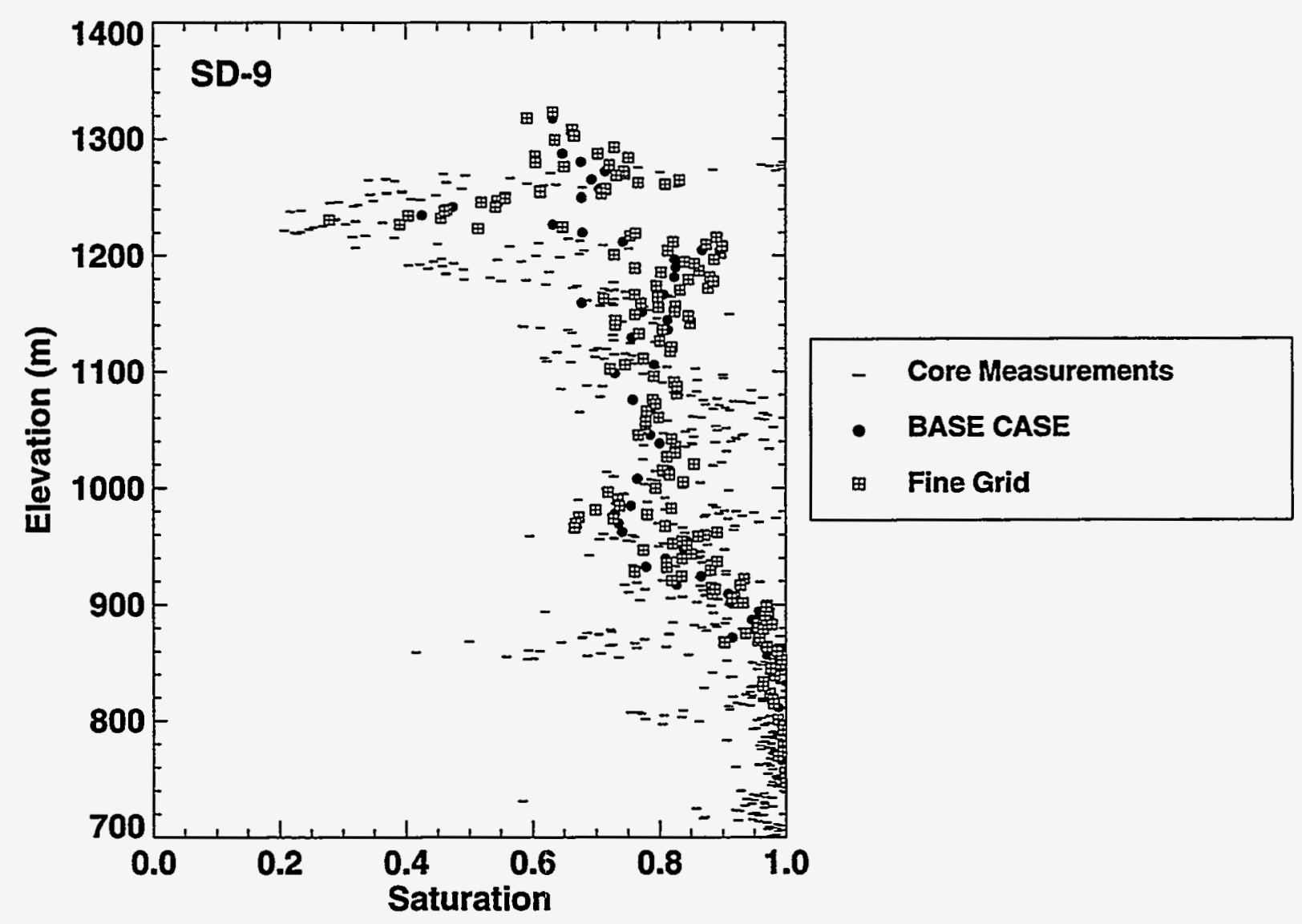

Figure 4-25 Comparison of simulated saturations to measured core saturations from SD-9 comparing the base case to a simulation using a finer grid.

Particle travel times vary from less than an order of magnitude to 2 orders of magnitude with longer travel times simulated when the finer grid is used (Figure 4-26). Differences in particle travel times can be attributed to the lower fracture saturations in the matrix domain. At the dry side of the relative permeability curve, small decreases in saturations will significantly decrease the fracture permeabilities and thus decrease the particle velocities. While flow velocities are clearly sensitive to the numerical gird, using a coarser grid results in more conservative estimates in particle travel times. 


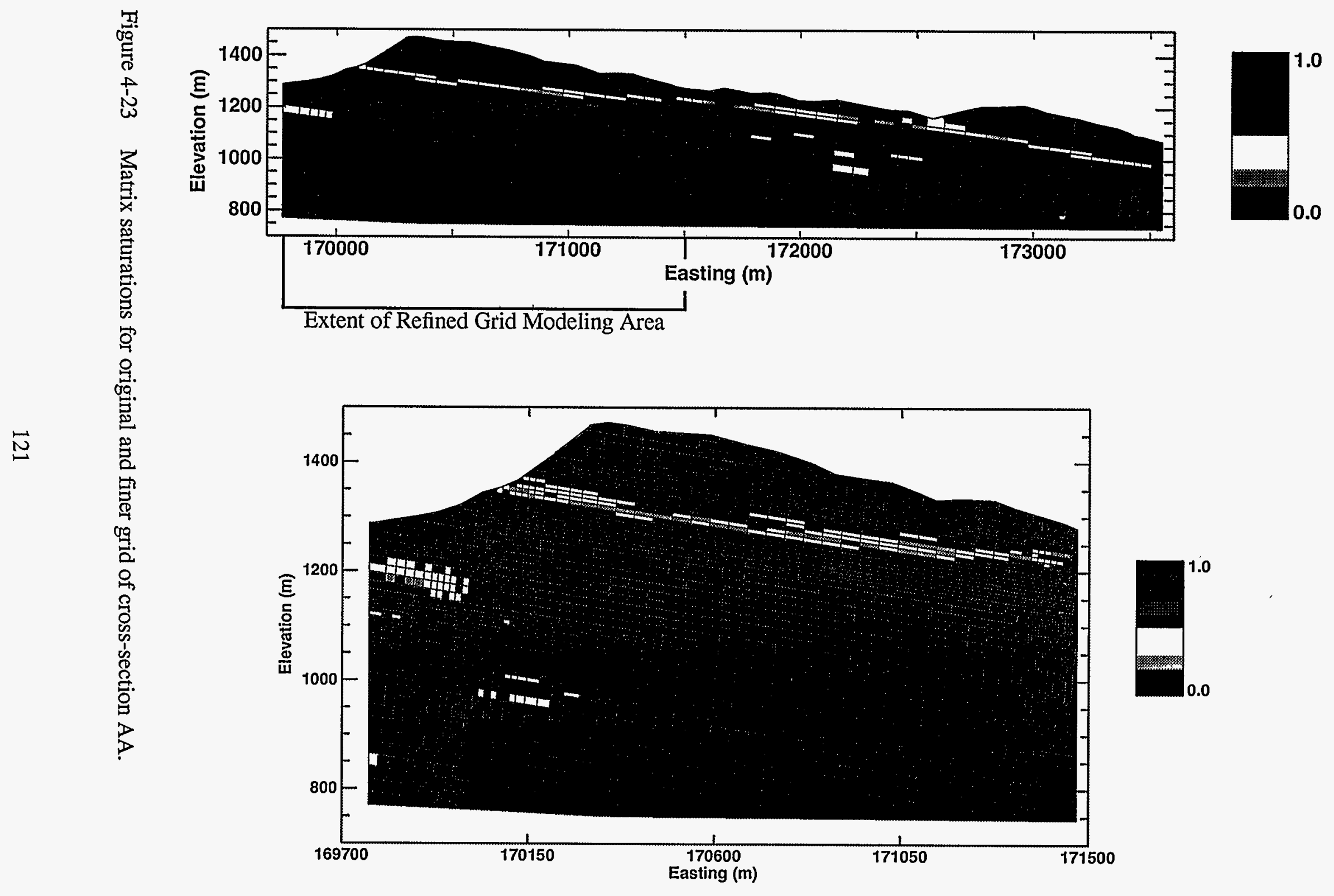



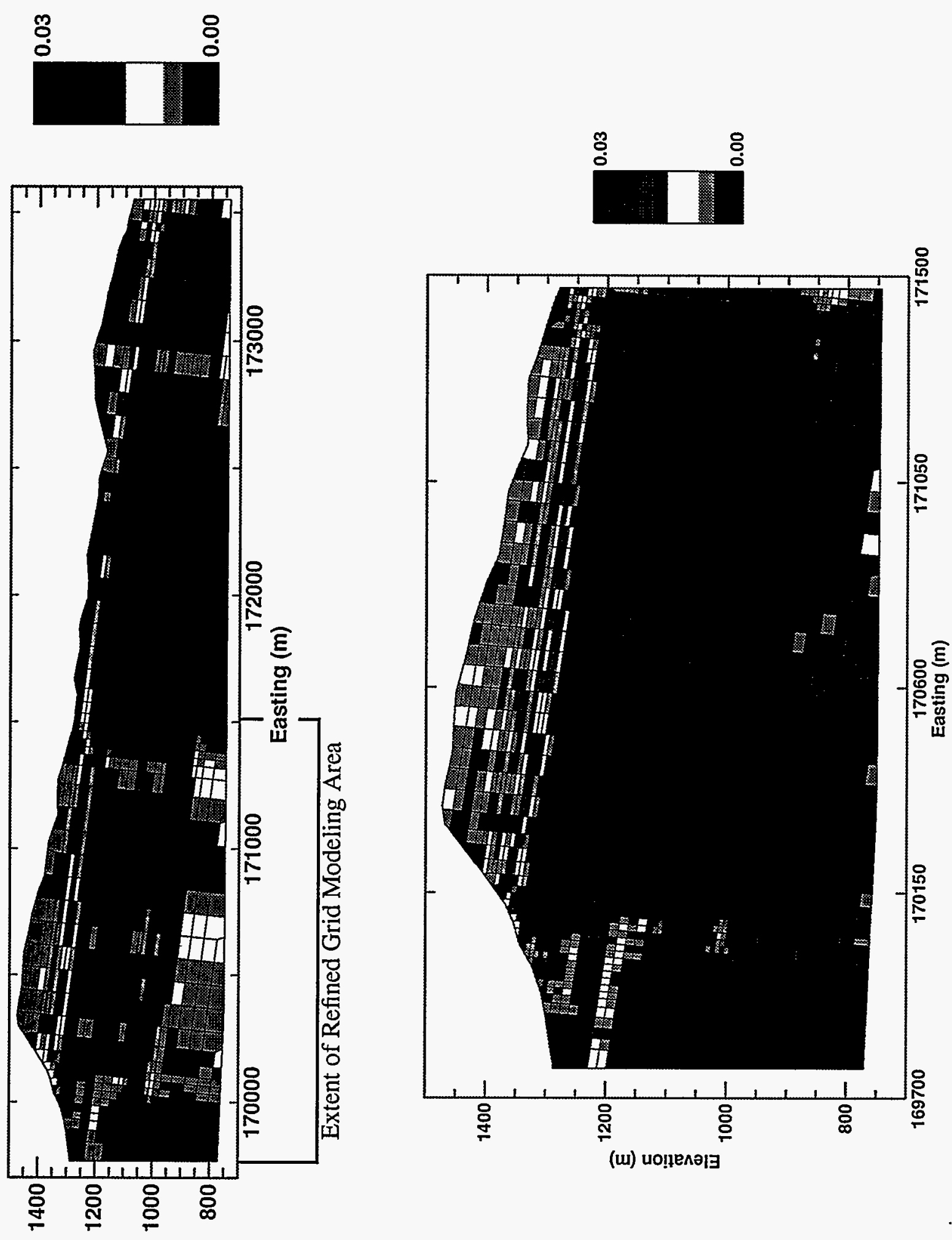

(u) นо!ฺеләㅋ

Figure 4-24 Fracture saturations for original and finer grid of cross-section AA. 


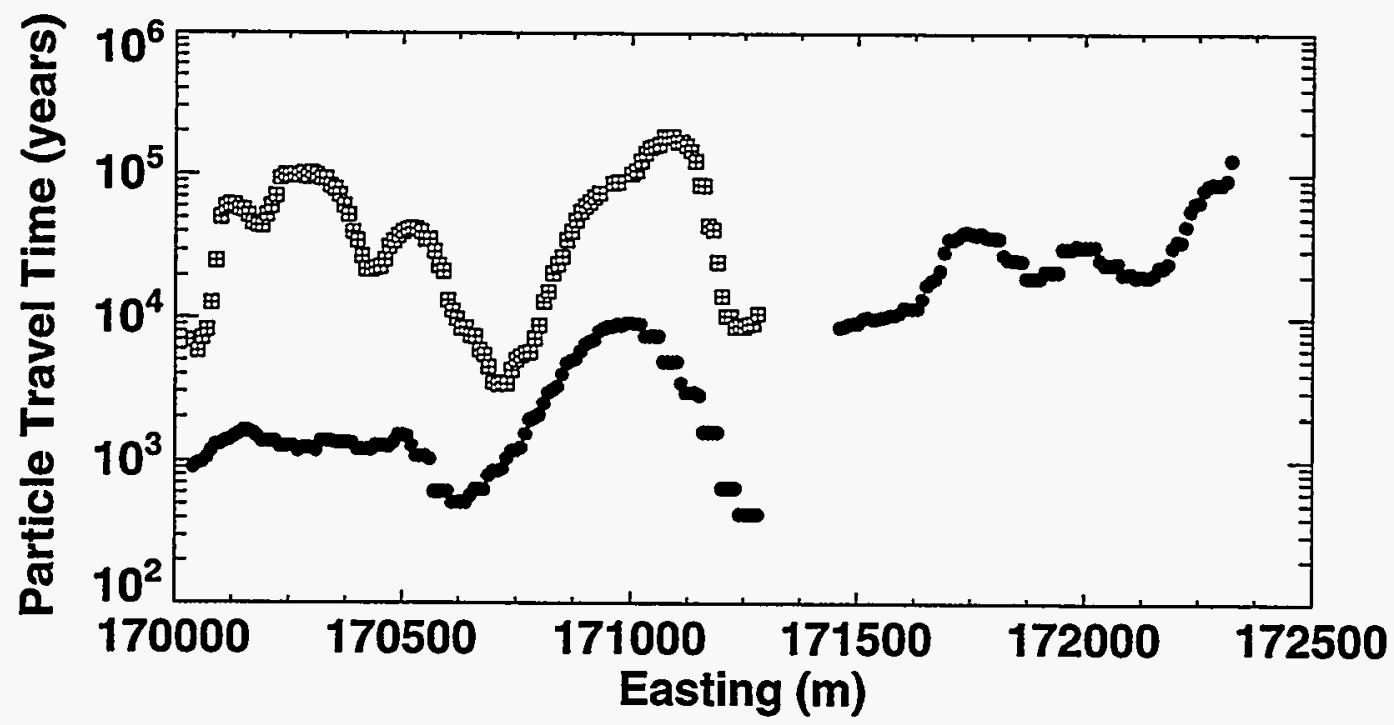

- BASE CASE
(⿴囗十) Grid

Figure 4-26 Particle travel times along cross-section AA for base case flow simulation and a simulations using a finer grid.

\subsubsection{Equivalent Continuum Model}

Previous Yucca Mountain modeling studies of unsaturated flow in fractured media have used the equivalent continuum model (ECM) (Section 2.3) (Prindle and Hopkins, 1990; Arnold et al., 1995; and Wittwer et al., 1995). To evaluate the effect of the different numerical implementation of the conceptual model, the ECM is used on the same parameter domain of Section A-A, realization 1. Although the ECM has just one combined domain, fracture and matrix saturations and fluxes are back-calculated at the end of the simulations from the separate characteristic curves.

Results demonstrate that the ECM simulated matrix saturations do not differ substantially from those generated using the DKM, except near the upper boundary (Figure 4-27). The largest differences are in the TCW; as explained previously, if more infiltration is introduced into the matrix domain of the TCw using the DKM, it would be possible to raise the saturations in this unit. Fracture saturations simulated using the ECM are lower than those simulated using the DKM (not shown). 


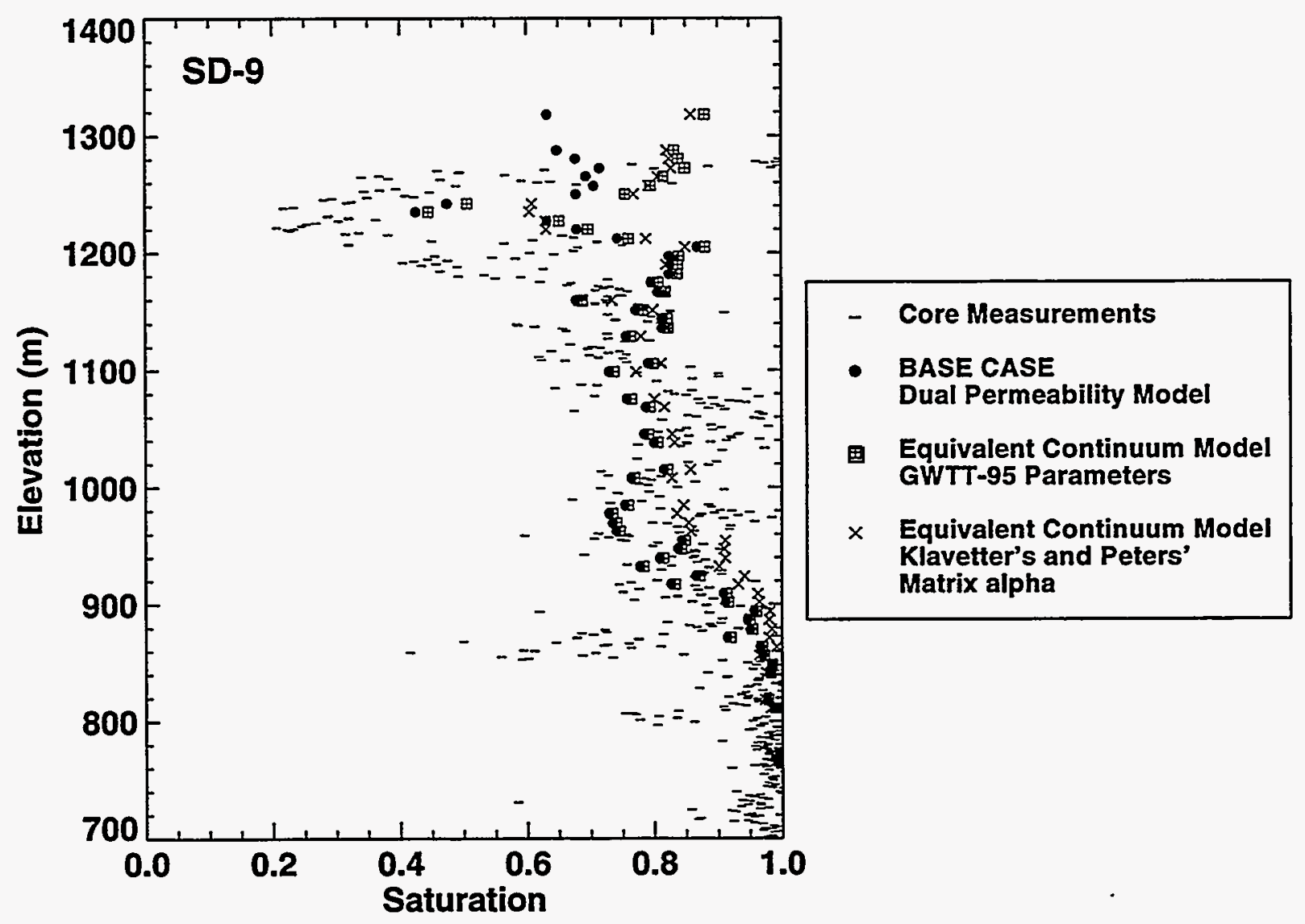

Figure 4-27 Comparison of simulated saturations to measured core saturations from SD-9 for simulations using the equivalent continuum and the dual permeability model.

Particle travel times are more than two orders of magnitude greater for the ECM than the DKM in most locations (Figure 4-28). Differences can be explained by the fluxes being higher in the fracture domain when the DKM is used; whereas, when the ECM is used the dominant fluxes are in the matrix and these are clearly less than that of the fracture domain in the DKM system.

\subsubsection{Matrix van Genuchten Parameters}

The range of matrix van Genuchten $\alpha$ used in GWTT-95 has changed significantly from previous studies (see Section 3.2.1.3) (Prindle and Hopkins, 1990, Wilson et al., 1994, Arnold et al., 1995, Wittwer et al., 1995). GWTT-95 calculations use more recent $\alpha$ values, which are thought to be more realistic because of improved measurement techniques and the inclusion of field data (Section 3.2.1.3). To evaluate the effects of the new van Genuchten $\alpha$ 's (and therefore new characteristic curves), simulations are run using the $\alpha$ values from Klavetter and Peters (1986). All other model parameters remained the same as those used in GWTT-95. A linear regression is used to determine $\alpha$ at each cell for the welded units, weighted by the relative area of that unit in the cross-section (Figure 4-29). A constant $\alpha$ of $1.6 \times 10^{-6} 1 / \mathrm{Pa}$ is used for the non- 


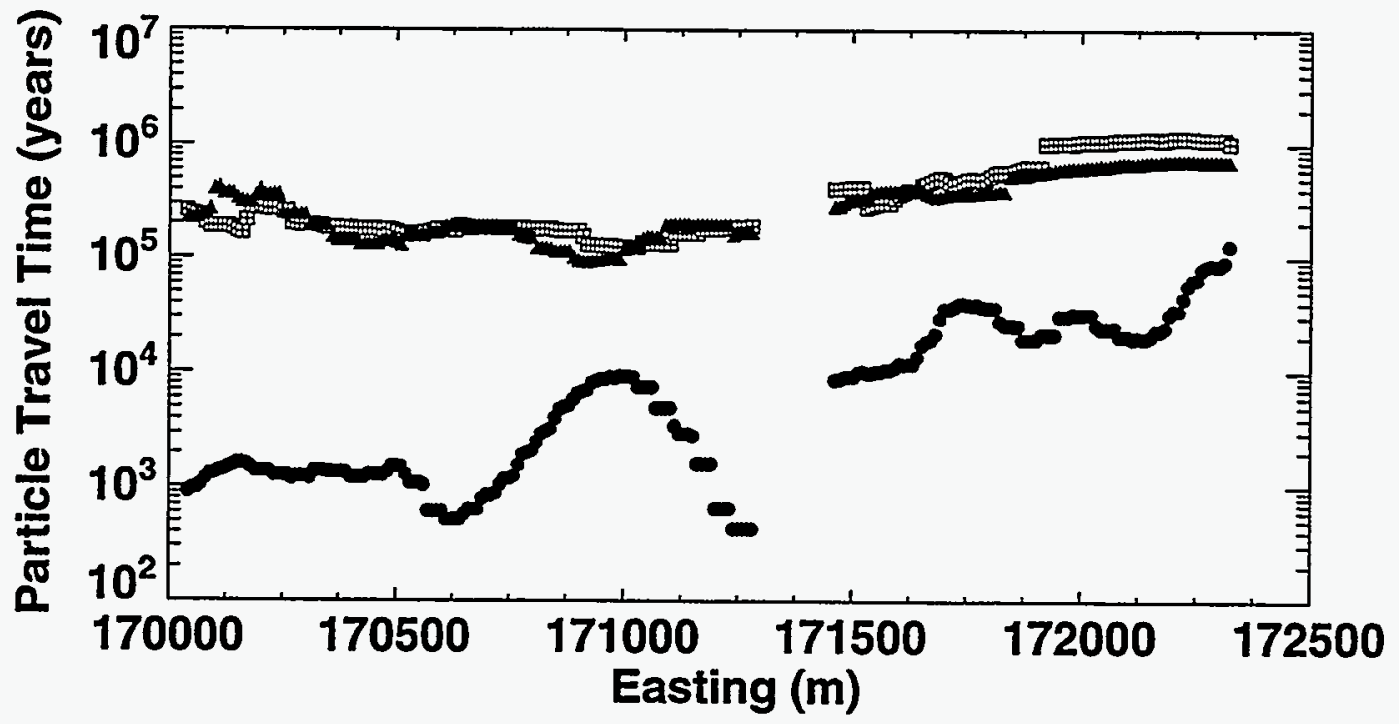

- BASE CASE

Dual Permeability Model

$\rightarrow$ Equivalent Continuum Model GWTT-95 alpha

- Equivalent Continuum Model

Klavetter and Peters (1986) alpha

Figure 4-28 Comparison of particle travel times along cross-section AA for simulations using the equivalent continuum (with two different sets of van Genuchten $\alpha$ ) and dual permeability models.

welded units. Welded and non-welded units are distinguished from each other by using a matrix porosity cut-off of 0.2 . The ECM is used in this sensitivity study to be consistent with the previous studies. Results from this simulation can be compared to those using the GWTT-95 parameters and the ECM model. Of particular interest is whether lateral diversion is observed in the Paintbrush nonwelded unit (PTn), as had been observed in other studies that used the parameters reported in Klavetter and Peters (1986) and (Prindle and Hopkins, 1990).

Comparison of matrix saturations between the two cases shows that saturations are somewhat higher when Klavetter's and Peters' $\alpha$ is used (Figure 4-27). Fracture saturations are also higher. when Klavetter's and Peters' $\alpha$ is used, most notably in the PTn. Lateral flow is observed in the PTn when the Klavetter's and Peters' $\alpha$ values are used, but the flow is primarily vertical throughout the system when the GWTT-95 parameters and the ECM are used (Figure 430). There are two factors influencing the lateral flow in the PTn. First the characteristic curves corresponding to the use of the Klavetter's and Peters' data are significantly different than that for the GWTT-95 data, so that lateral diversion can occur under drier conditions due to higher suction pressure in the PTn (Wilson, 1996). Second, the effects of using a combined fracture/matrix 


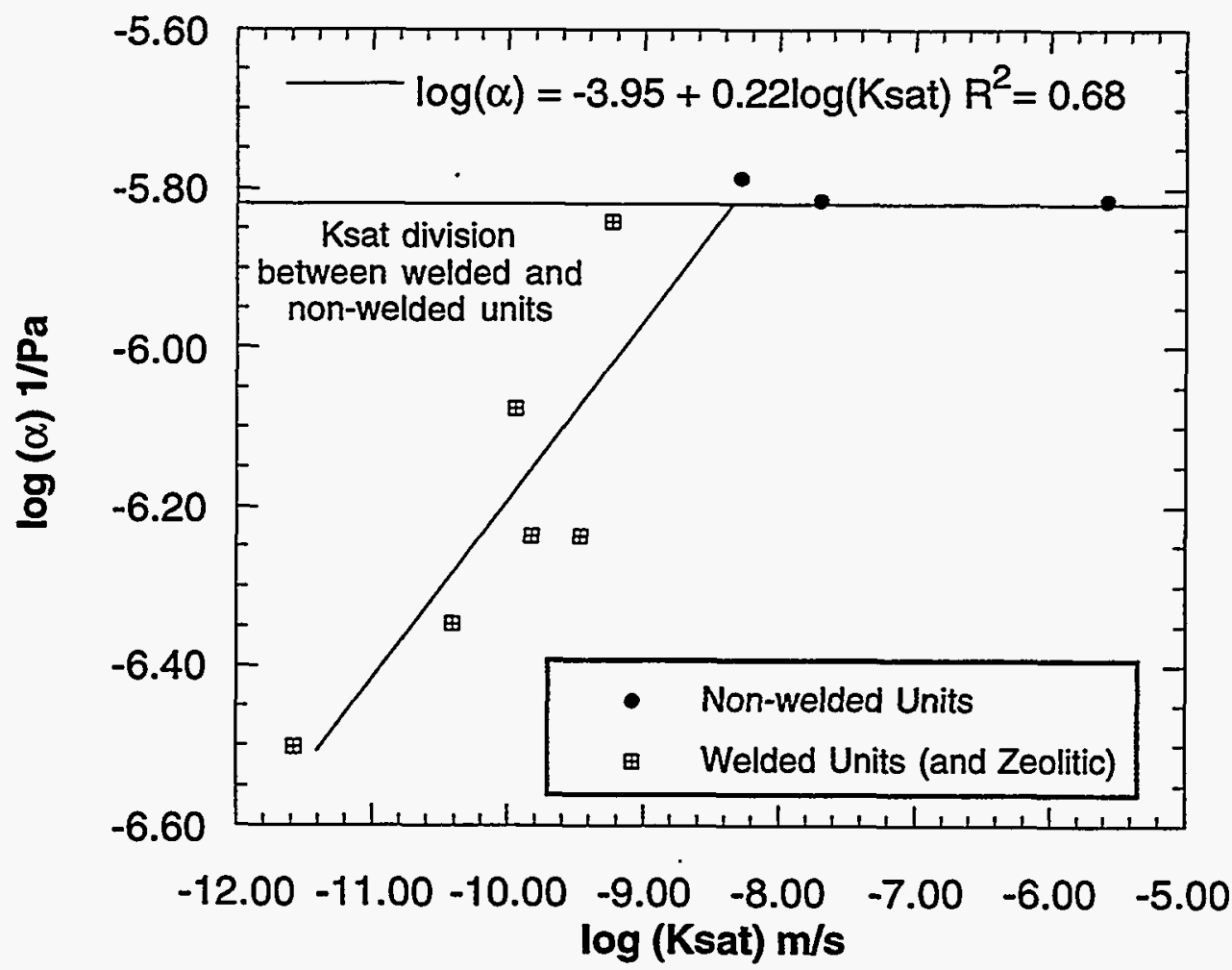

Figure 4-29 Relationship between Ksat and van Genuchten $\alpha$ used in simulating $\alpha$ 's using Klavetter's and Peters' data.

characteristic curve for the ECM significantly decreases the suction pressure in the TSw, again resulting in lateral flow in the PTn under drier conditions (Klavetter and Peters, 1986). This sensitivity study has shown that Klavetter's and Peters' van Genuchten $\alpha$ values are necessary for the water to flow laterally. It is also likely that the ECM plays a role in promoting lateral flow.

As a result of this lateral flow when the Klavetter's and Peters' $\alpha$ is used, the particle travel times increase slightly in most locations (Figure 4-28). The lateral diversion decreases the flux at the repository horizon where the particles are released. The decrease is especially noticeable under the PTn outcrop. 


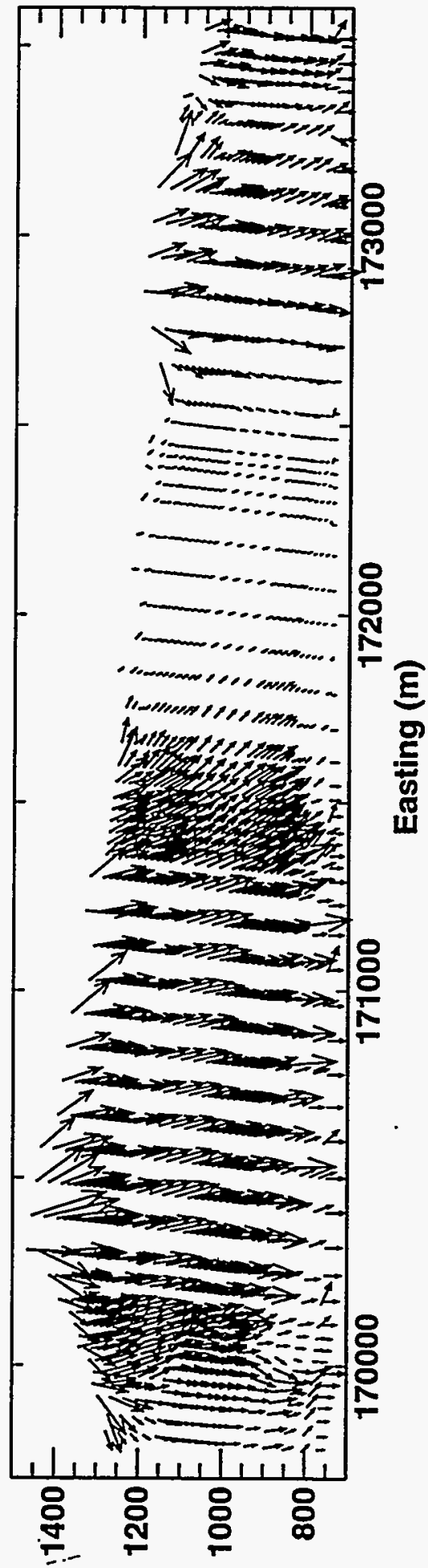

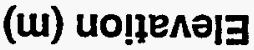

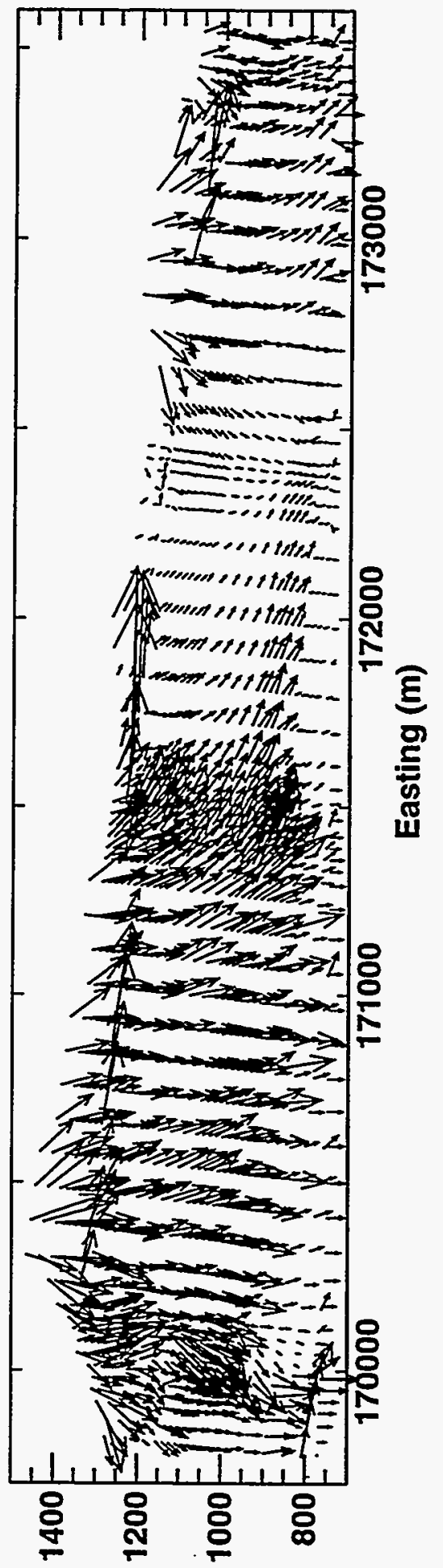

(w) Uo!̣jen키

Figure 4-30 Flow velocities through fractures for two simulations both using the equivalent continuum model, but one using GWTT-95 van Genuchten Parameters and the second using van Genuchten parameters from Klavetter and Peters (1986). 


\subsubsection{Episodic Infiltration}

Previous simulations in this study have been performed with steady infiltration distributions, primarily for convenience and a lack of dependable data on future temporal distributions of infiltration. However, episodic events that yield short pulses of high infiltration are likely to be important in producing fast-flow paths in the unsaturated zone. The potential effects of an episodic infiltration event are investigated in this section by arbitrarily increasing the baseline infiltration distribution over cross-section AA (Realization 1) for a short period of time.

The identical dual permeability model used in the previous steady-state simulations is used in this analysis. However, for one week, the infiltration (applied to the fracture continuum) is increased by nearly four orders of magnitude as shown in Figure 4-31. The total infiltration during this one-week event is approximately several centimeters of rain (i.e. a feasible amount for a series of rainstorms in a pluvial period). Following this event, the baseline infiltration distribution is reapplied for 10,000 years. The initial condition for this sequence of events is taken from the steady-state runs presented earlier.

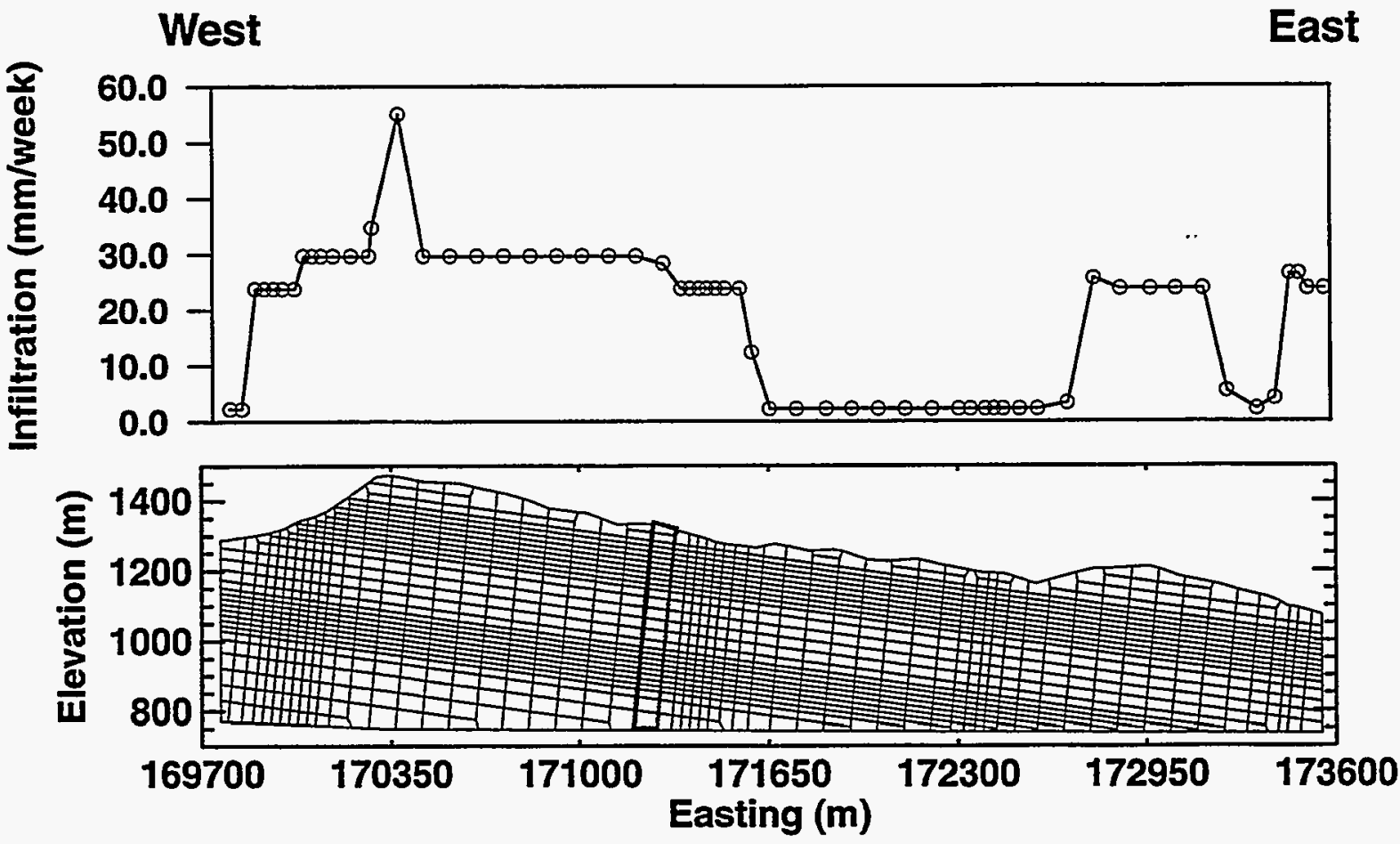

Figure 4-31 Episodic infiltration rate applied to cross-section AA for one week. The effects of the episodic event is analyzed along a single column of elements that is outlined in bold. 
The fracture saturation, matrix saturation, and fracture velocity are recorded after each time step along a nearly vertical column of elements (highlighted in Figure 4-31) to investigate the effects of the infiltration pulse with time. Figure 4-32 shows a qualitative assessment of the effect of the infiltration pulse on the fluid pore velocities in fractures (herein referred to as fracture pore velocities). Darker shades represent a greater increase in the fracture pore velocity (from the steady-state value) as a function of elevation and time. The infiltration pulse is felt strongly near the surface at very early times while the infiltration pulse is being applied ( $<1$ week). Although it is greatly attenuated after passing through the PTn, the pulse propagates to the bottom of the domain after only 30 years. This is better quantified by plotting the fracture pore velocities at four locations (the surface $(y=1,318 \mathrm{~m}), \operatorname{PTn}(y=1,242 \mathrm{~m})$, repository horizon $(y=1,106 \mathrm{~m})$, and bottom $(y=773 \mathrm{~m})$ ) as a function of time as shown in Figure 4-33. The moisture contents at these four locations are also plotted in a similar fashion in Figure 4-34. These plots indicate that this one-week infiltration event impacts the fracture pore velocity and saturation at great depths within a relatively short period of time. It is also interesting to note that the matrix saturation remains relatively unchanged during this episodic event (Figure 4-35).
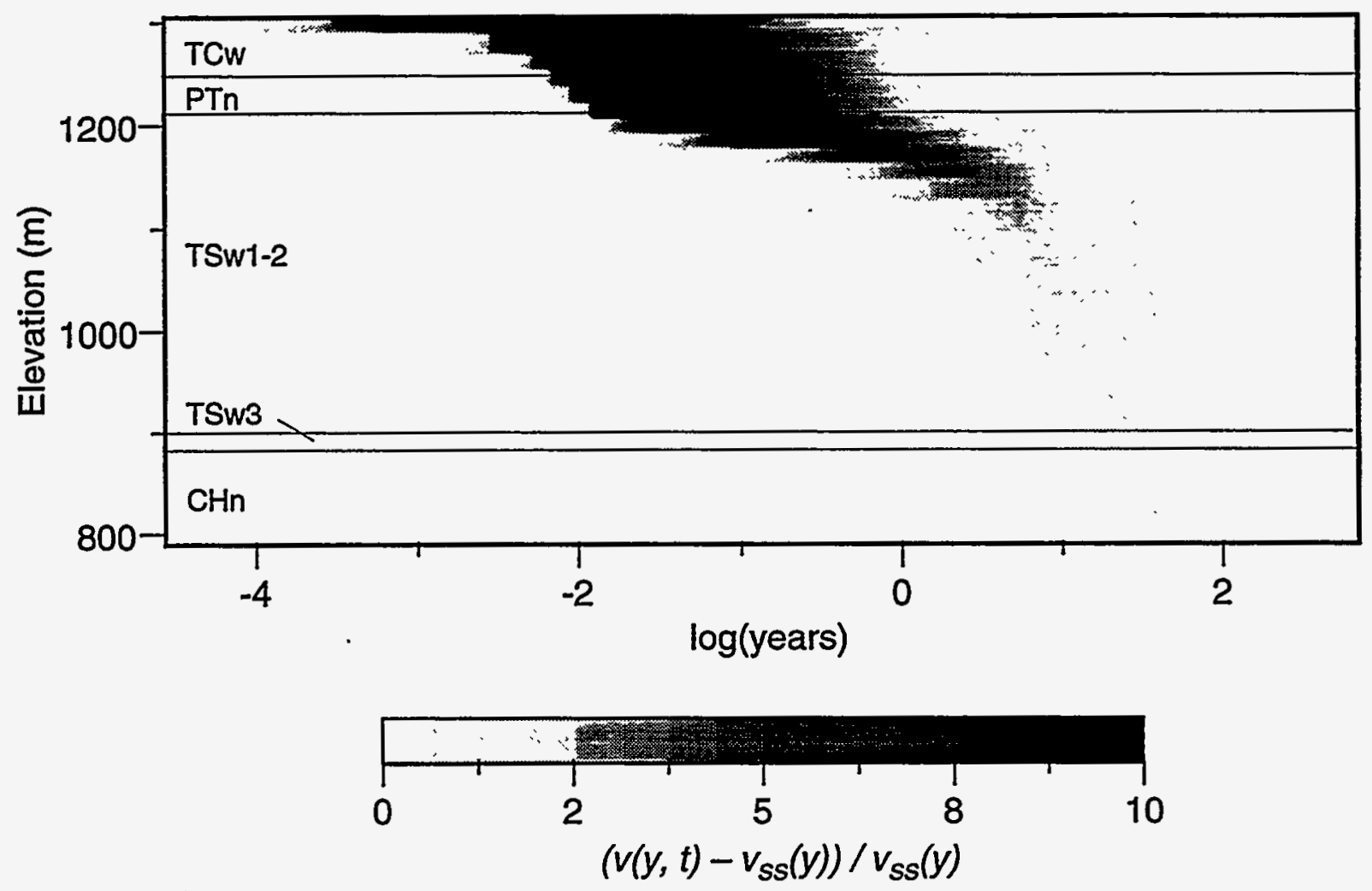

Figure 4-32 Relative increase in fracture pore velocity, $v$, over the steady-state value, $v_{s s}$, as a function of elevation and time since the initiation of the infiltration pulse (note the log scale) for one column of elements as a result of the one week episodic infiltration event. 


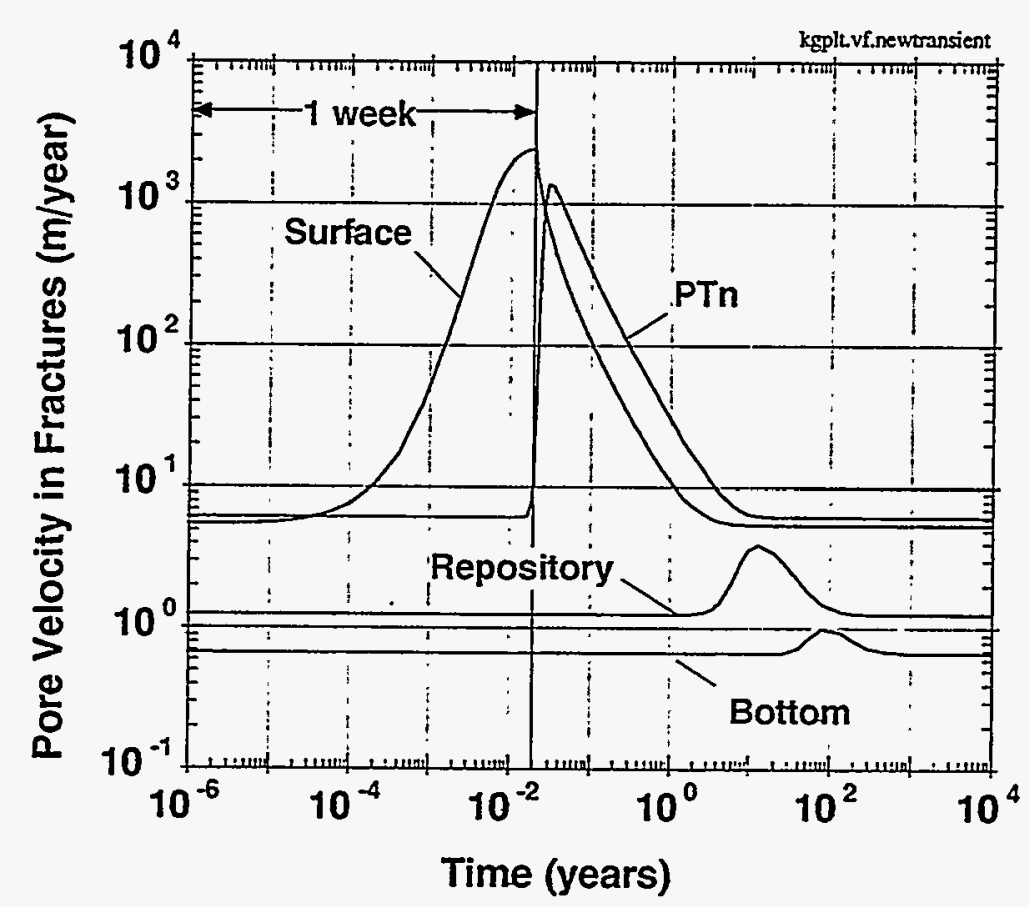

Figure 4-33 Fracture pore velocity as a function of time at four locations along a column of elements in section AA as a result of a one-week episodic infiltration pulse.

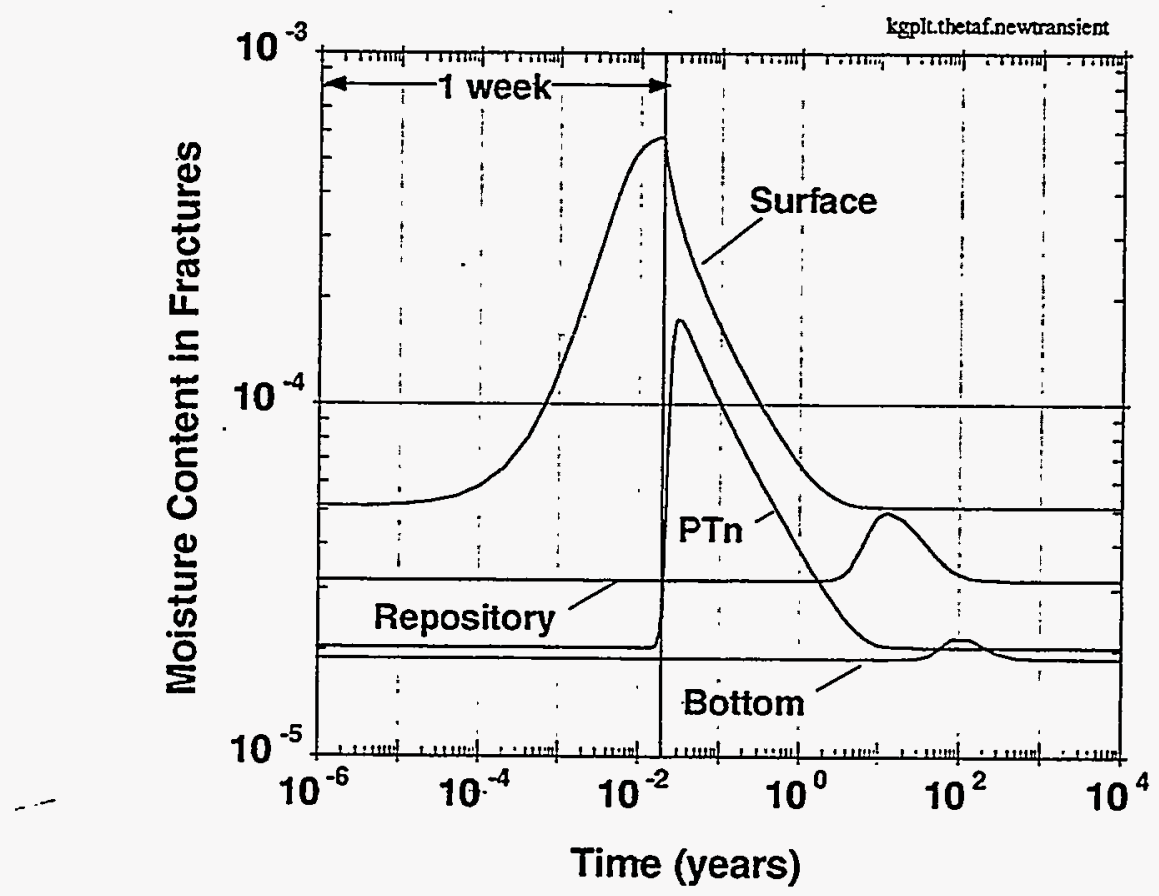

Figure 4-34 Fracture water content (liquid saturation times porosity) as a function of time at four locations along a column of elements in section AA as a result of a oneweek episodic infiltration pulse. 


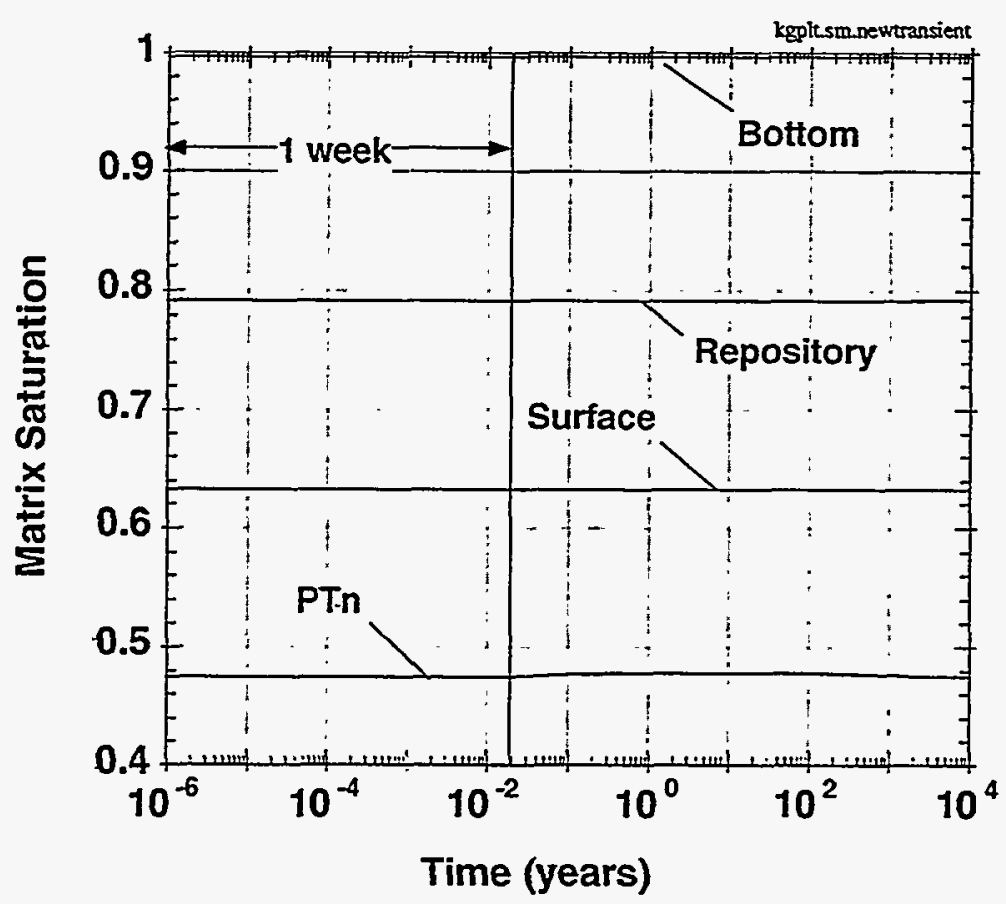

Figure 4-35 Matrix saturations as a function of time at four locations along a column of elements in section $\mathrm{AA}$ as a result of a one-week episodic infiltration pulse.

The mass of water, $m$, passing through the fractures in each of the four locations as a result of this event can be calculated in the following manner. At a given elevation, $y$, the fracture pore velocities, $v(y, t)$, (Figure 4-33) are multiplied by the corresponding fracture moisture contents, $\theta(y, t)$, (Figure 4-34) to yield a Darcy velocity, which is then integrated with respect to time ( $\Delta t=10,000$ years). To obtain the contribution of the one-week pulse only, the steady-state component is then subtracted from the previous quantity (steady-state parameters are denoted by the subscript, $s s)$. The result is then multiplied by the liquid density $\left(\rho=998 \mathrm{~kg} / \mathrm{m}^{3}\right)$ and crosssectional area of flow $\left(A \sim 76 \mathrm{~m}^{2}\right)$ to yield the mass of water that passes through the fractures at a given location as a result of the infiltration pulse.

$$
\text { - } m(y)=\rho A(y)\left(\int_{0}^{\Delta t}(v(y, t) \theta(y, t)) d t-v_{s s}(y) \theta_{s s}(y) \Delta t\right)
$$

Equation (4-13) can also be expressed as a fraction of the total mass of water introduced at the top of the column of elements by dividing equation (4-13) by the total amount of water that was infiltrated at the top of the column, $m_{g}$, during the one-week infiltration pulse $\left(m_{g}=2269 \mathrm{~kg}\right)$. 
Both the total mass of water and the fractional amount of water passing through the fractures at each of the four locations are shown in Table 4-3. Note that the fractional flux of water through the fractures as a result of the infiltration pulse is greatly attenuated below the PTn as a probable result of significant imbibition into the matrix elements in the PTn unit. Only $22 \%$ of the water introduced at the top of the boundary reaches the repository horizon through the fractures as a result of the infiltration pulse. Nevertheless, Table 4-3 shows that even a one-week infiltration pulse within a 10,000 year period can increase the amount of water flux through the fractures in the current conceptual model. Longer durations and more frequent episodes would act to increase the amount of flow through the fractures at greater depths.

Table 4-3: Total and fractional amount of water flowing through the fractures at four locations as a result of the one-week infiltration pulse.

\begin{tabular}{lcc}
\hline & $\begin{array}{c}m(y)(\mathrm{kg}) \\
\text { (Eq. (4-9)) }\end{array}$ & $m(y) / m_{g}$ \\
\hline surface $^{\dagger}(\mathrm{y}=1,318 \mathrm{~m})$ & 2,115 & 0.932 \\
PTn $(\mathrm{y}=1,242 \mathrm{~m})$ & 1,188 & 0.524 \\
repository horizon $(\mathrm{y}=1,106 \mathrm{~m})$ & 502 & 0.221 \\
bottom $(\mathrm{y}=773 \mathrm{~m})$ & 167 & 0.0737 \\
\hline
\end{tabular}

†This denotes flow from the surface element to the element directly beneath it.

Although the infiltration pulse propagates rather quickly through the system, the actual travel time of a water particle can be significantly different. The travel times of particles released from the surface, PTn, and repository horizon are investigated by tracking the movement of a particle of water as advected by the fracture pore velocity at each time step (dispersion and matrix imbibition are not included). Figures $4-36 \mathrm{a}$ and $4-36 \mathrm{~b}$ show a sequence of plots that track the movement of these particles of water. Note that the particle released at the surface "rides" with the same velocity as the infiltration pulse at very early times, but it begins to drop behind the pulse at later times. This is especially true as the infiltration pulse passes through the PTn, where significant imbibition into the matrix occurs (recall that the particles are only being tracked in the fractures). Nevertheless, the particle released at the surface reaches the PTn in less than one year, which is consistent with recent isotopic dating results that show possible evidence of bomb pulse $\left({ }^{36} \mathrm{Cl}\right)$ in the $\mathrm{PTn}{ }^{30}$. Without the infiltration pulse, simulated steady-state travel times from the surface to the PTn would exceed 10 years at that location. Similarly, these simulations indicate that a particle released at the repository horizon would travel about $100 \mathrm{~m}$ in 40 years following introduction of the pulse, but would travel about $30 \mathrm{~m}$ under steady-state conditions without the pulse.

30. Fabryka-Martin, J., personal communication, 1995. 

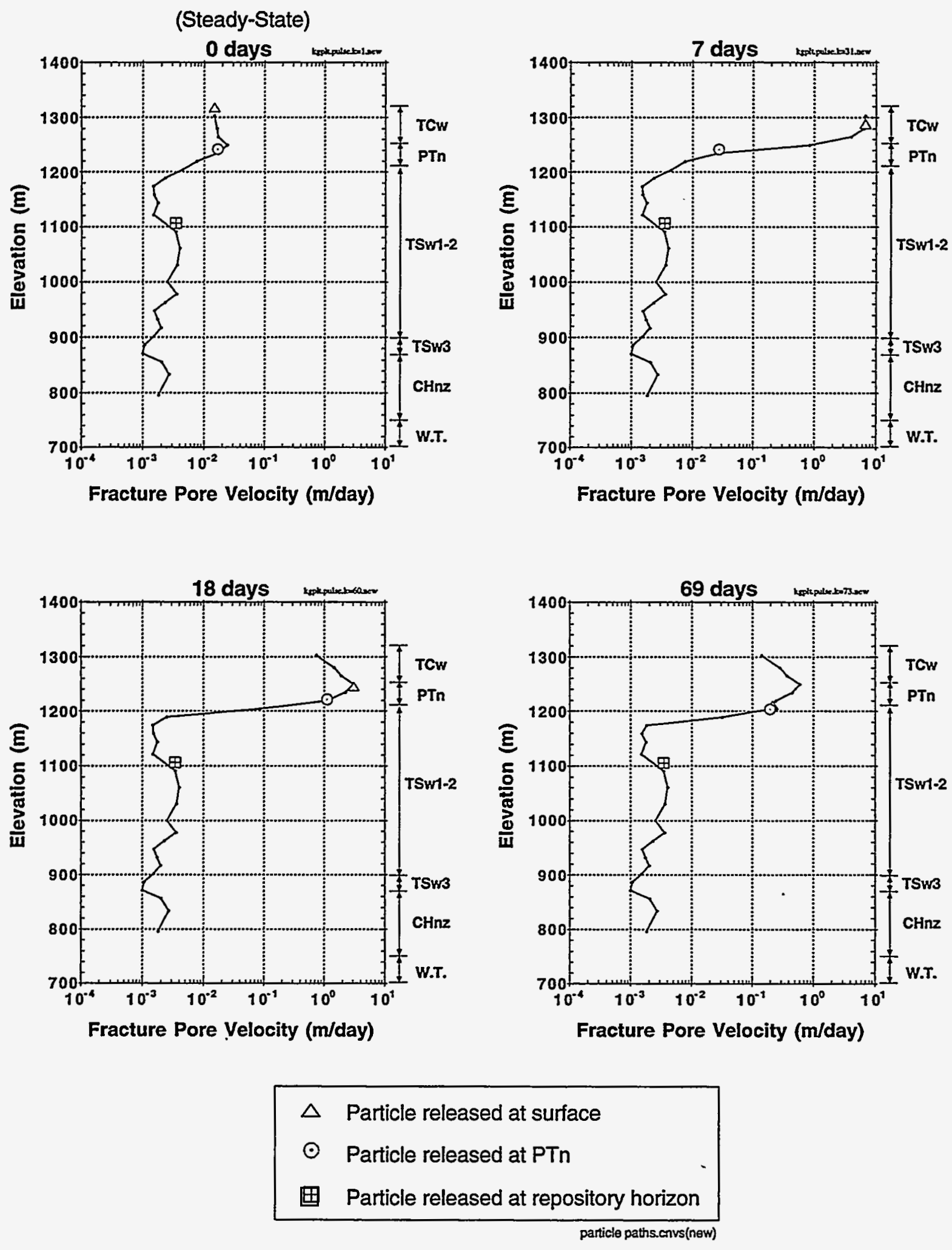

Figure 4-36a Particle movement through a single column of fracture elements as a result of a one-week infiltration pulse in section AA. The plots show the position of water particles (denoted by symbols) at various times during and following the infiltration pulse. The fracture pore velocities are also shown to indicate the effects of the infiltration pulse. 

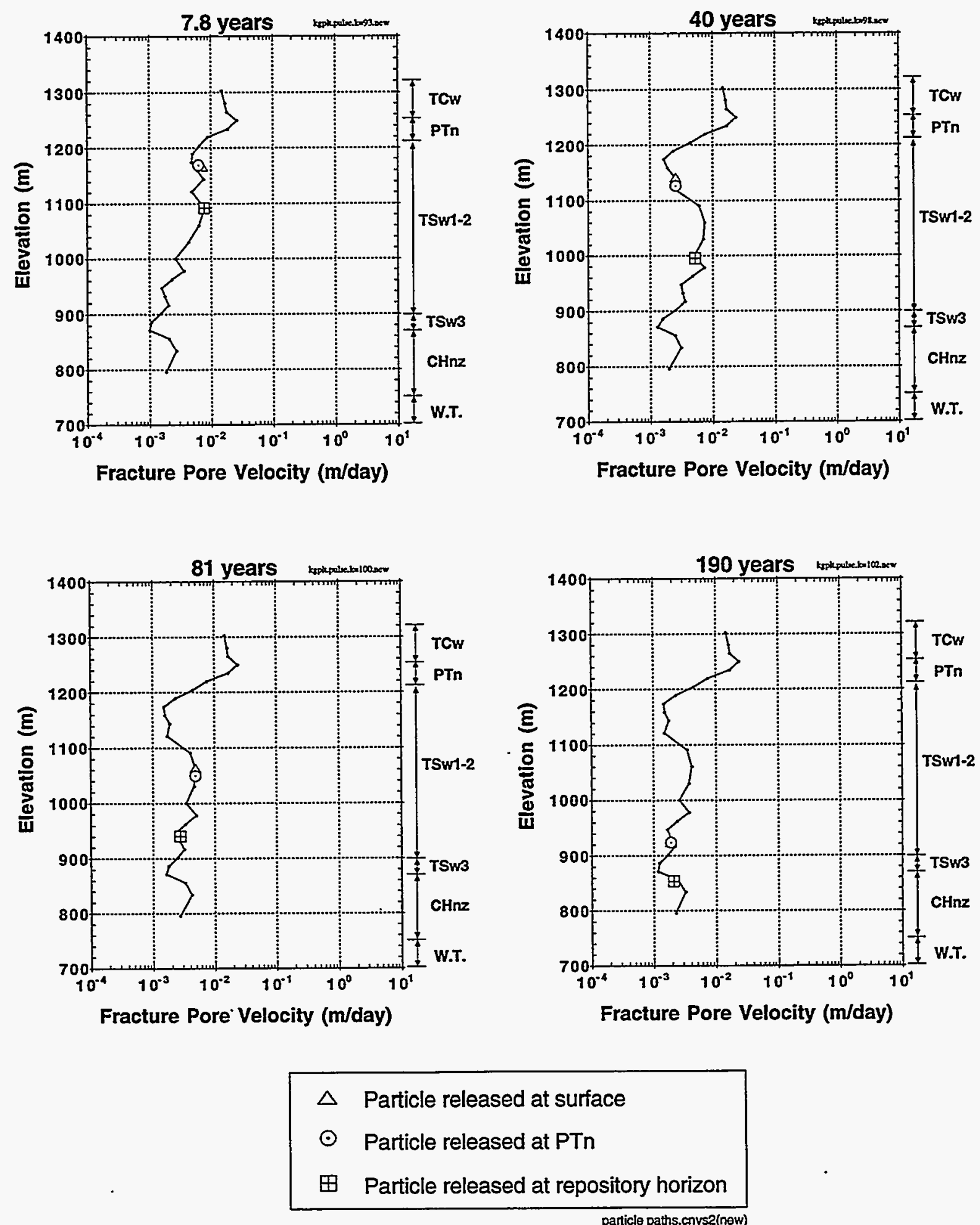

Figure 4-36b Particle movement through a single column of fracture elements as a result of a one-week infiltration pulse in section AA. The plots show the position of water particles (denoted by symbols) at various times during and following the infiltration pulse. The fracture pore velocities are also shown to indicate the effects of the infiltration pulse. 
It is important to remember that these results are from a single, one-week infiltration pulse over a ten-thousand year period. Longer durations and larger magnitudes of these episodic infiltration events could result in even larger water fluxes and faster travel times to greater depths in the fractures. In addition, the travel times in faulted regions may be faster than the travel times observed for the unfaulted column of elements chosen in this study as a result of greater fracture permeabilities. Therefore, based on the large apparent impact of these episodic infiltration events on water flux and travel times in the unsaturated zone, future investigations should attempt to consider episodic infiltration events to complement the conventional steady-state infiltration boundary. 
This page intentionally left blank. 


\section{CHAPTER 5 \\ Numerical Flow Modeling In The Saturated Zone}

In contrast to the stochastic flow modeling approach taken in the UZ analysis, a single representation of the geologic framework and homogeneous intra-unit material properties are used in the SZ modeling. This approach is taken primarily because of the relative lack of detailed and accurate information on hydrologic properties controlling flow in the $\mathrm{SZ}$ for use as conditioning data. However, random variability of flow in the SZ is incorporated through simulation of hydrodynamic dispersion in the particle tracking within the flow field.

This chapter describes the assumptions and implementation of the SZ flow model. The calibration of the flow model and conclusions reached from the calibration process are also presented. The particle tracking method, which is used to determine the distribution of groundwater travel time resulting from the simulated flow field, is described, along with conclusions regarding the spatial and temporal distributions of travel time.

\subsection{Flow Model Domain}

The areal extent of the SZ flow model is shown in Figure 5-1. The lateral boundaries of the model are extended relative to the boundaries used in Wilson et al. (1994) to further isolate the simulated flow field in the vicinity of the repository from the effects of the specified-head boundary conditions. Additional motivation for extending the boundaries include the desire to incorporate the Yucca Wash structure in the model and alignment of the eastern boundary with Forty Mile Wash. The upper $250 \mathrm{~m}$ of the SZ are contained in the flow model. The geologic framework model employed in the flow model is presented in Section 3.1.2 of this report.

\subsubsection{Hydrostratigraphic Units}

The upper portion of the SZ within the modeled area occurs within Cenozoic volcanic units of the Paintbrush Group and Crater Flat Group. Hydrostratigraphic units included in the model are the Topopah Spring Tuff, Calico Hills Formation, Prow Pass Tuff, Bullfrog Tuff, and Tram Tuff (see Table 2-1). In addition, the regions to the north of the Yucca Wash structure and to the west of Windy Wash fault are designated as separate hydrologic units. These two regions are assigned homogeneous material properties, owing to the lack of subsurface geologic data in these areas.

Hydrostratigraphic units are distinguished primarily on the basis of degree of welding and, by inference, fracture density and fracture permeability. A correlation between degree of welding (as indicated by matrix porosity) and fracture permeability is suggested by the parameter development work of this study (see Section 3.2.2.2) and by field measurements of air permeability (LeCain and Walker, 1994). Some minor adjustments to stratigraphic classification are made to honor this conceptual basis for hydrostratigraphic unit designation. For example, the 


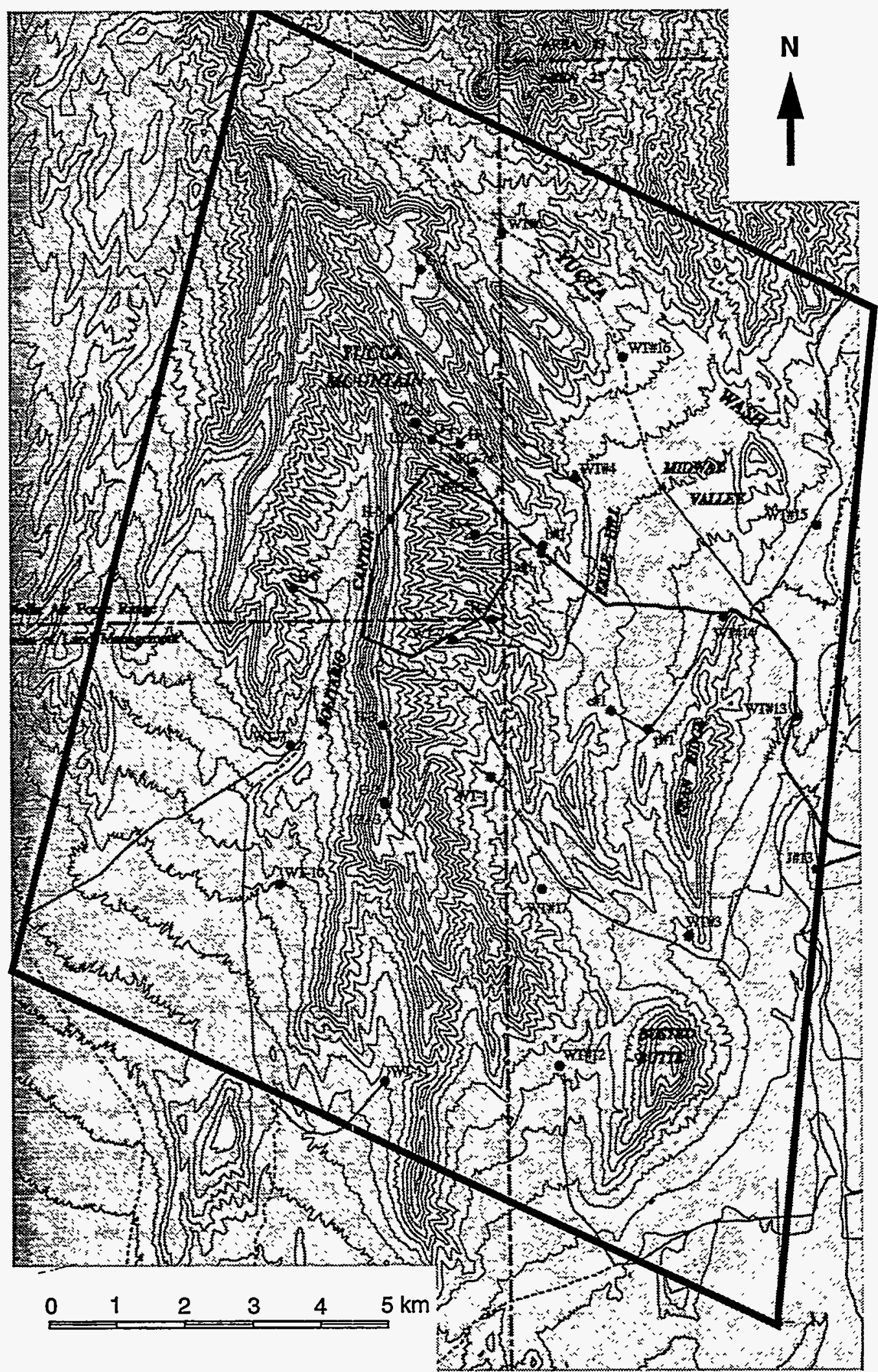

Figure 5-1 Areal extent of the saturated zone flow model (bold line) and locations of wells intersecting the water table. Contours indicate elevation of the topographic surface. Contour interval is $100 \mathrm{ft}$. 
nonwelded tuff at the base of the Topopah Spring Tuff is included in the Calico Hills Formation hydrostratigraphic unit because of its inferred hydrologic affinity with that unit. The hydrostratigraphic units employed in this study approximately correspond to those defined in Wilson et al. (1994).

\subsubsection{Boundary Conditions}

The lateral boundaries of the flow model are specified-head boundaries. The values of specified head assigned to the bounding nodes are taken, in a general sense, from the results of two-dimensional regional-scale flow modeling (Czarnecki and Waddell, 1984; D'Agnese, 1994; Faunt, 1994). Lateral boundary heads are interpolated from contour plots of head from these regional studies. Values of head at the boundaries are adjusted along the southwestern boundary of the flow model in this study to achieve reasonable calibration with some wells on the western side of the Solitario Canyon fault. The same value of hydraulic head is specified for all layers at a given location on the boundary, implying no vertical flow at the boundaries. The upper and lower surfaces are no-flow boundaries. These boundary conditions are consistent with the conceptual model of flow in the saturated zone described in Section 2.4.2.

\subsection{Numerical Simulation of Flow}

Simulation of the SZ flow system, as outlined by the conceptual model and incorporating specific features of the model domain and boundary conditions, is accomplished by numerical techniques. The simulation process includes a trial-and-error method of model calibration in which parameters are varied to meet criteria that have been established to judge the validity of the flow model. The resulting groundwater flow model is a self-consistent representation of the physical system.

\subsubsection{Governing Equations}

The governing equation for steady-state groundwater flow in a saturated, heterogeneous, confined aquifer is given by:

$$
\frac{\partial}{\partial x_{i}}\left[K_{i j} \frac{\partial h}{\partial x_{j}}\right]=0
$$

where $K_{i j}$ is the saturated hydraulic conductivity tensor of the equivalent porous medium, $h$ is the hydraulic head, and $x_{i}(\mathrm{i}=1,2,3)$ are the three orthogonal spatial coordinates.

Several assumptions about the physical system are embodied in this governing equation. It is assumed that an equivalent hydraulic conductivity tensor exists for the fractured medium. This assumption is supported by the relatively high fracture density observed in boreholes and outcrops for most of the units. This assumption can be partially verified by well testing, such as 
that planned for the C-well complex (Geldon, 1993). An additional assumption is isothermal conditions and constant fluid density. This assumption is a valid approximation for prevailing conditions, but must be relaxed for future calculations of post-emplacement flow in the SZ.

\subsubsection{Numerical Grid}

The finite-element grid used to obtain the numerical solution to flow in the $\mathrm{SZ}$ consists of quadrilateral elements in five planar layers. A total of 13,800 nodes and 11,025 elements constitute the mesh. The nodal coordinates were adjusted in the regions of wells at which head measurements are available, so nodes correspond to well locations (see Figure 5-2). Material properties are assigned to elements based on the prevailing hydrostratigraphic unit within the element (see discussion of the geologic framework model in Section 3.1.2 and Figure 3-5).

\subsubsection{Numerical Solution Using STAFF3D}

Numerical simulation of groundwater flow in the SZ was performed using the STAFF3D code (Huyakorn et al., 1992) by the finite-element method. It is used here to solve the steadystate, saturated flow problem described above. The Galerkin formulation is used for the groundwater flow equation. Solution to the resulting matrix problem is achieved iteratively using the preconditioned conjugate gradient method.

\subsection{Flow Model Calibration and Results}

The first step in calibration of the groundwater flow model is to establish the criteria most relevant to the problem of interest for evaluating the calibration. The most directly pertinent measurements are the values of hydraulic head at the 27 wells (from Ervin et al., 1993) located within the model domain (see Figure 5-1). These data are chosen as the primary criteria for evaluating calibration. It should be noted, however, that many of these wells are open over large vertical intervals below the water table. These wells represent a vertically integrated measure of hydraulic potential and should, thus, be interpreted with caution. A second set of criteria for evaluating the flow model is the general direction and magnitude of the hydraulic gradient in the area to the east and south of the potential repository. These criteria are directly relevant to the calculation of groundwater travel time in the region down-gradient from the repository. The inferred direction (east and southeast) and magnitude (0.0001) of the hydraulic gradient are taken from Ervin et al. (1993) and Fridrich et al. (1994).

The calibration process revealed that several discrete structural features may exert considerable influence on the groundwater flow field and inclusion of these features in the flow model results in a much better calibration of the model. These features include the Solitario Canyon fault, the Solitario Canyon fault splay in the region of drillhole H-5, the Drill Hole Wash structure, and the Yucca Wash structure. The locations of these features in the flow model are shown in Figure 5-3. The reduction of hydraulic conductivity by one to three orders of magnitude relative to geologically equivalent units results in the contrasts in hydraulic potential observed in 


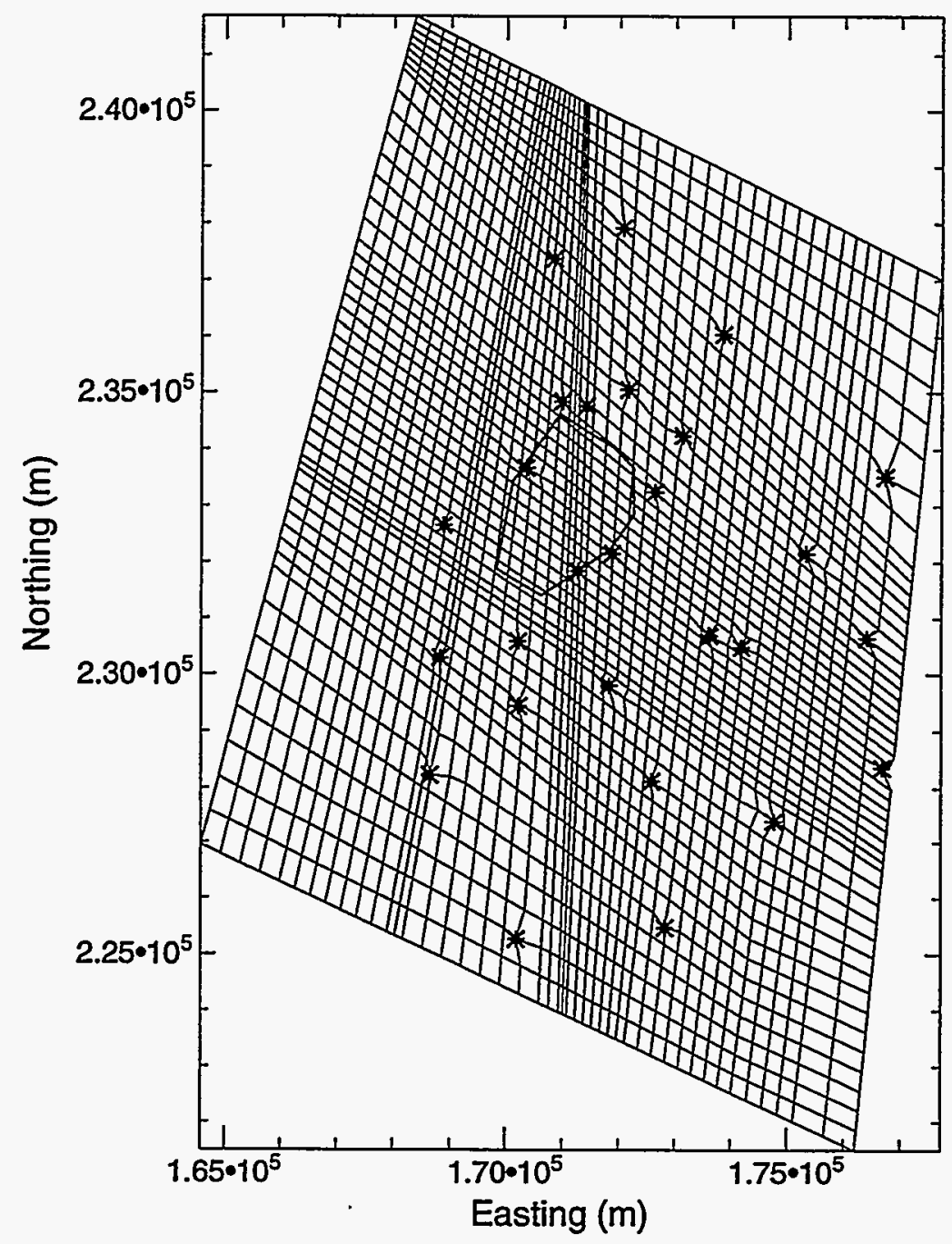

Figure 5-2 Numerical grid for the STAFF3D flow model of the saturated zone. Water table.wells used in the calibration of the model are shown with asterisks. Coordinates are based on the Nevada State Plane system.

wells located on opposite sides of the structural features. These structural features, of unknown hydraulic properties, have been mapped or inferred from geologic mapping at the surface (Scott and Bonk, 1984).

The calibrated flow model shows an acceptable match with well data and simulates the general direction and magnitude of hydraulic gradient in the area to the east and south of the potential repository (see Figure 5-4). The values of hydraulic conductivity used in the calibrated model are given in Table 5-1 and a comparison of simulated and measured hydraulic head is tabulated in Table 5-2. The values of hydraulic conductivity assigned to the TSw unit are approximately equivalent to the expected value of fracture permeability for this unit in the UZ 


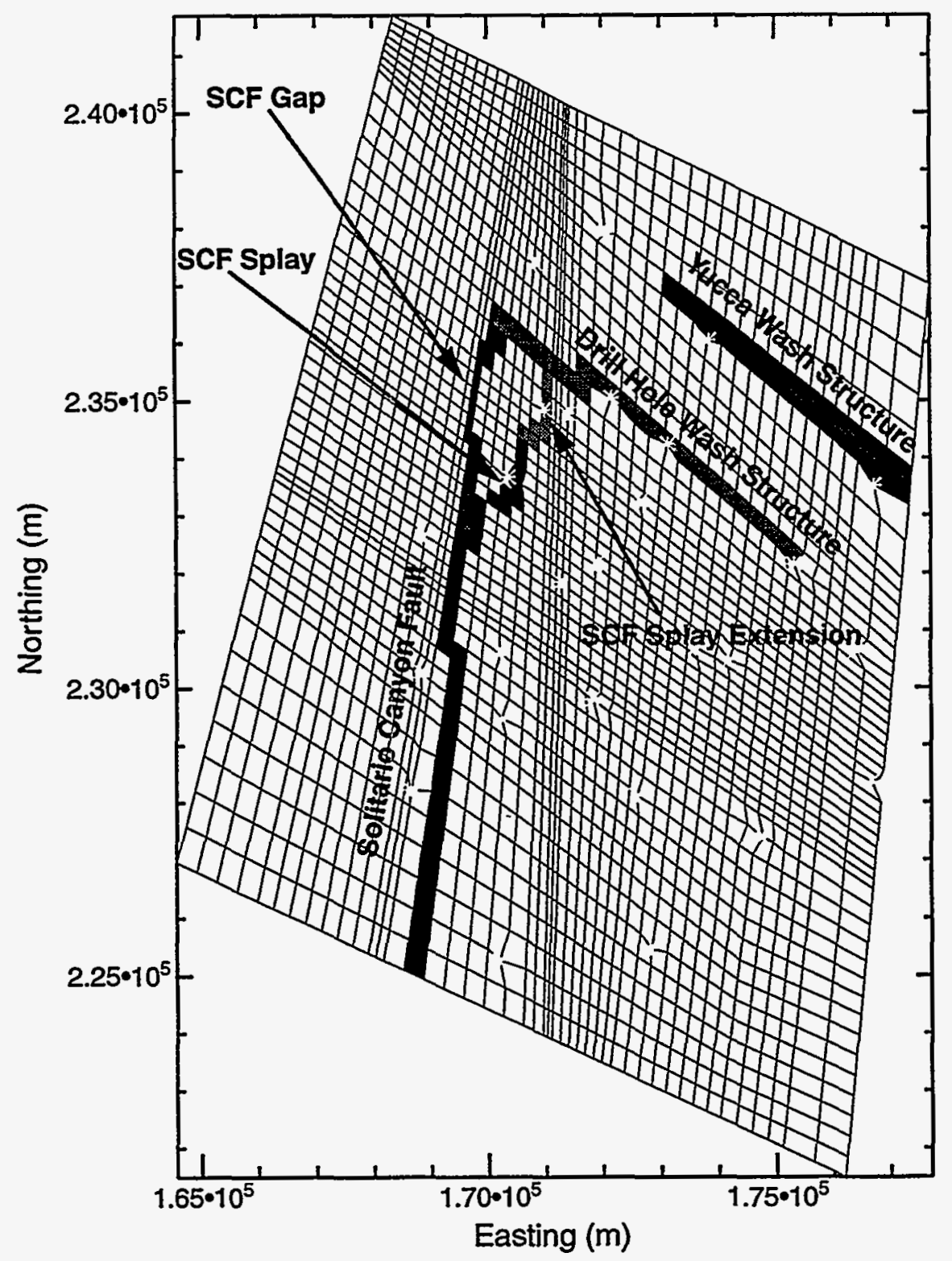

Figure 5-3 Discrete structural features included in the calibrated SZ flow model.

flow modeling. The expected value of fracture permeability for the $\mathrm{CH}$ unit in the UZ modeling is about one order of magnitude higher than the value of hydraulic conductivity assigned to this unit in the SZ modeling. However, the few borehole air permeability measurements available for the CHn (LeCain and Walker, 1994) indicate that the lower permeability (as used in the SZ modeling) is probably more accurate. Values of hydraulic conductivity used in the SZ modeling for the Prow Pass and Bullfrog units are somewhat higher than the corresponding fracture permeability values used in the UZ modeling. It should be noted that the Topopah Spring unit and the area to the north of Yucca Wash are divided into subunits (designated as Topopah Spring(2) and North(2) in Table 5-1). These subunits correspond to a small volume to TSw near borehole G-2 and the northeastern corner of the model domain (see Figure 3-5). These subunits are defined 
to improve the calibration of the flow model in these areas. Simulated values of hydraulic head are within $1.0 \mathrm{~m}$ of the observed head at wells downgradient from the repository, with the exceptions of wells WT-13, WT-14, and WT-15. There are significant discrepancies between the flow model and observed head at well H-5, G-1, G-2, and WT-16. Well G-2 is located in the region of the large hydraulic gradient and on the margin of subsurface geologic control. G-2 is also located in an area that may be affected by significant vertical flow to the deeper Paleozoic carbonate aquifer (Fridrich et al., 1994), which is not considered in this flow model. Wells H-5, G-1, and WT-16 are all located near the low-conductivity faults. The present flow model probably lacks the spatial resolution and structural complexity to accurately model the head in these regions. Because these discrepancies are deemed to have little impact on the groundwater travel time calculations in this study they are left unresolved.

Table 5-1: Hydraulic parameters used in the calibrated flow model.

\begin{tabular}{|l|l|}
\hline \multicolumn{1}{|c|}{ Unit } & Hydraulic Conductivity $(\mathrm{m} / \mathrm{s})$ \\
\hline \hline Topopah Spring(1) & $5.00 \times 10^{-5}$ \\
\hline Topopah Spring(2) & $1.25 \times 10^{-6}$ \\
\hline Calico Hills & $1.25 \times 10^{-6}$ \\
\hline Prow Pass & $8.00 \times 10^{-4}$ \\
\hline Bullfrog & $5.00 \times 10^{-4}$ \\
\hline Tram & $2.00 \times 10^{-7}$ \\
\hline North(1) & $5.00 \times 10^{-6}$ \\
\hline North(2) & $1.00 \times 10^{-5}$ \\
\hline West & $1.25 \times 10^{-6}$ \\
\hline Solitario Canyon fault & $1.00 \times 10^{-8}$ \\
\hline Drill Hole Wash Structure & $1.00 \times 10^{-7}$ \\
\hline SCF Gap & $5.00 \times 10^{-7}$ \\
\hline SCF Splay & $1.00 \times 10^{-7}$ \\
\hline SCF Splay Extension & $1.20 \times 10^{-5}$ \\
\hline Yucca Wash Structure & $5.00 \times 10^{-8}$ \\
\hline
\end{tabular}

Table 5-2: Comparison of simulated and measured heads for the calibrated flow model.

\begin{tabular}{|l|c|c|c|c|}
\hline _. Well \# & Node \# & $\begin{array}{c}\text { Measured } \\
\text { Head }(\mathrm{m})\end{array}$ & $\begin{array}{c}\text { Simulated } \\
\text { Head }(\mathrm{m})\end{array}$ & $\begin{array}{c}\text { Residual } \\
(\mathrm{m})\end{array}$ \\
\hline \hline WT-11 & 112 & 730.72 & 729.86 & -0.86 \\
\hline WT-10 & 290 & 775.92 & 776.36 & +0.44 \\
\hline WT-12 & 310 & 729.52 & 729.13 & -0.39 \\
\hline WT-7 & 473 & 775.70 & 777.39 & +1.69 \\
\hline G-3 & 525 & 730.56 & 729.76 & -0.80 \\
\hline
\end{tabular}




\begin{tabular}{|l|c|c|c|c|}
\hline \multicolumn{1}{|c|}{ Well \# } & Node \# & $\begin{array}{c}\text { Measured } \\
\text { Head }(\mathrm{m})\end{array}$ & $\begin{array}{c}\text { Simulated } \\
\text { Head }(\mathrm{m})\end{array}$ & $\begin{array}{c}\text { Residual } \\
(\mathrm{m})\end{array}$ \\
\hline \hline WT-17 & 584 & 729.64 & 729.11 & -0.53 \\
\hline H-3 & 662 & 731.72 & 730.10 & -1.62 \\
\hline WT-3 & 684 & 729.57 & 728.82 & -0.75 \\
\hline WT-1 & 718 & 730.40 & 729.69 & -0.71 \\
\hline H-6 & 885 & 775.96 & 777.33 & +1.37 \\
\hline WT-2 & 1036 & 730.71 & 730.26 & -0.45 \\
\hline J-13 & 1150 & 728.45 & 728.45 & 0.00 \\
\hline H-4 & 1177 & 730.33 & 730.14 & -0.19 \\
\hline C-3 & 1185 & 730.10 & 729.60 & -0.50 \\
\hline P-1 & 1233 & 730.00 & 729.71 & -0.29 \\
\hline H-5 & 1260 & 775.47 & 732.82 & -42.65 \\
\hline B-1 & 1502 & 730.66 & 730.19 & -0.47 \\
\hline G-1 & 1538 & 754.20 & 733.33 & -20.87 \\
\hline H-1 & 1588 & 730.95 & 730.49 & -0.46 \\
\hline WT-13 & 1609 & 728.98 & 730.41 & +1.43 \\
\hline WT-18 & 1683 & 730.80 & 730.51 & -0.29 \\
\hline WT-4 & 1687 & 730.70 & 730.50 & -0.20 \\
\hline WT-14 & 1697 & 729.71 & 734.74 & +5.03 \\
\hline G-2 & 1812 & 1029.00 & 983.01 & -45.99 \\
\hline WT-16 & 2011 & 738.32 & 812.43 & +74.11 \\
\hline WT-15 & 2023 & 729.24 & 732.68 & +3.44 \\
\hline WT-6 & 2050 & 1035.10 & 1030.34 & -4.76 \\
\hline & & & & \\
\hline
\end{tabular}

The simulated potentiometric surface as calculated by the flow model for the upper layer is shown in Figure 5-4. Because of the extreme variability in hydraulic gradient across the area, the general distribution of head is shown by the surface in the upper portion of the figure and the contours of head for only the low hydraulic gradient region are shown in the lower part of the figure. The important role of the faults in controlling the simulated flow field is illustrated in Figure 5-4. The Drill Hole Wash structure and the Yucca Wash structure correspond to the areas of very steep gradient and the Solitario Canyon fault produces a head differential of about $40 \mathrm{~m}$. The direction of flow indicated by the potentiometric surface is generally south and east from the potential repository. The magnitude of the hydraulic gradient directly to the southeast of the repository is approximately $1.4 \times 10^{-4}$. These simulated results provide an adequate match to the secondary calibration criteria stated above (magnitude and direction of the hydraulic gradient).

There are several qualifying considerations regarding the SZ flow model presented in this report. This model incorporates a single conceptualization of the flow system, in which the primary control on flow is the distribution of low-conductivity fault zones and secondary control is exercised by the geometry of the hydrostratigraphic units. Vertical flow into and out of the upper $250 \mathrm{~m}$ of the SZ is not considered, though measurements of temperature in wells suggests that there may be significant hydraulic interaction with the underlying Paleozoic carbonate aquifer (Sass et al., 1988). Because of the low hydraulic gradient in the critical region to the southeast of the repository, the problem of model calibration is poorly constrained in this area (i.e. values of simulated head are relatively insensitive to values of hydraulic conductivity chosen for 


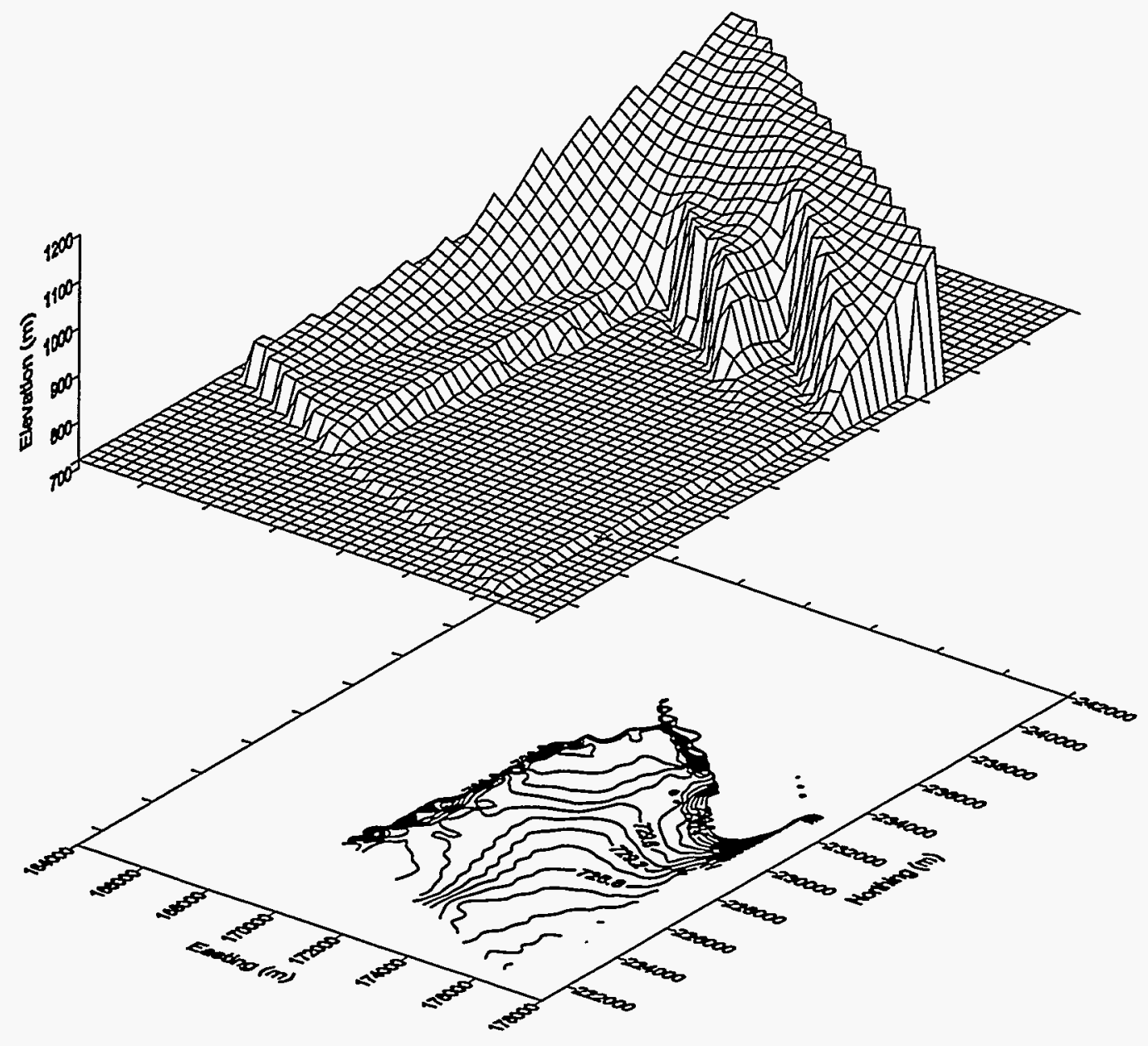

Figure 5-4 Simulated hydraulic head in the upper layer of the calibrated SZ flow model. The upper part of the figure shows the potentiometric surface over the entire model in perspective view. The lower figure is a contour plot of head only in the region of the low hydraulic gradient to the east and south of the repository. The contour interval in the lower figure is $0.2 \mathrm{~m}$.

this area). Furthermore, the low hydraulic gradient makes it difficult to "see" the influence of possibly important features downgradient of the repository. For example, an increase in the hydraulic gradient associated with lower conductivity of the Bow Ridge fault would be below the resolution of the available head measurements. These uncertainties could result in considerable inaccuracies in the simulated direction and magnitude of groundwater velocity in the flow model. 


\subsection{Particle Tracking}

A method of particle tracking within the simulated flow field was developed to determine the distribution of groundwater travel time from the water table below the repository to the accessible environment. The definition of the accessible environment used here is a boundary five kilometers from the potential repository.

\subsubsection{Particle Transport Processes}

Particles are conceptualized to undergo advection, hydrodynamic dispersion, and matrix diffusion in the groundwater flow system. Advection simply occurs at the ambient fluid flow velocity. Dispersion of particles occurs by a random Fickian process due to variations in velocity and flow-path length at scales smaller than the flow grid. Matrix diffusion is assumed in an implicit manner. Although groundwater flow is conceptualized to occur primarily in fractures, molecular diffusion of water particles into the matrix will lead to complete mixing between fracture water and matrix water, given a sufficiently long flow path and time. Calculations by Robinson (1994) for conditions typical of the SZ at Yucca Mountain indicate complete molecular diffusion of a solute into the matrix for groundwater travel times greater than about 100 years. Because travel times to the five $\mathrm{km}$ boundary are expected to exceed 100 years, complete exchange of water particles between the fractures and matrix is assumed and a value of effective porosity representative of matrix porosity was chosen for the flow model. A value of porosity of 0.20 is assigned to all units. This uniform value of porosity is used for all units due to lack of data from the SZ on effective porosity.

\subsubsection{Implementation of Particle Tracking}

A particle tracking scheme consistent with the finite-element method used in the flow modeling was developed for use in conjunction with the output of the STAFF3D code. The first step in modeling advective transport is to interpolate the gradient of hydraulic head from the head at surrounding nodes. The groundwater velocity at the nodes is then calculated using the hydraulic conductivity and porosity of the element, and Darcy's Law. The velocity field within the element is represented by tri-linear interpolation using the basis function for linear parallelepiped elements. In essence, the velocity field in the global coordinate system is "mapped" onto the local coordinate system of the finite-element grid using the inverse of the Jacobian matrix. For elements that are nearly parallelograms, the Jacobian is nearly constant and the particle path within the element can be calculated using a semianalytical solution (Pollock, 1988).

The process of hydrodynamic dispersion is simulated in the particle tracking procedure by the random-walk method. Although discrete time steps are not required to calculate advective movement by the semianalytical method, the particle tracking of advection is halted following specific time intervals and a random displacement is added to the particle's position. The additional displacement is randomly drawn from a Gaussian distribution with the variance defined as: 


$$
\sigma^{2}=2 v \alpha_{i} \Delta t
$$

where $v$ is the velocity, $\alpha_{i}$ is the dispersivity (longitudinal and transverse), and $\Delta t$ is the time step. The displacement has two components, one in the longitudinal direction and one in the transverse direction. Longitudinal dispersivity of $40 \mathrm{~m}$ is used for all units in the particle tracking calculations. This value of longitudinal dispersivity is somewhat larger than that based on a theoretical estimate for the TSw unit (see section 3.2.2.5) because of the relatively larger scale of flow modeling elements. A value one order of magnitude less $(4 \mathrm{~m})$ is used for transverse dispersivity.

\subsubsection{Particle Tracking Results}

Particles are placed at the water table directly below the repository for tracking to the five $\mathrm{km}$ boundary of the accessible environment. The pathlines for a limited number of particles are shown in Figure 5-5. Flow is generally to the southeast as indicated by the potentiometric contour map in Figure 5-4, with some random component to the particle pathlines due to dispersion. Note that the pathlines shown are projected onto the map view and that there is considerable variability of the pathlines in the vertical dimension.

Groundwater flow in the region downgradient of the repository in the model is controlled by the geometry of the hydrostratigraphic units and the contrasts in hydraulic conductivity among the units. An important feature of the geology in the shallow SZ is the presence of a block of Calico Hills Formation on the downthrown side of the Bow Ridge fault to the east and southeast of the repository, as shown in brown in Figure 3-5. This zone of low conductivity material, which becomes thinner with depth, diverts flow to the south and under the Calico Hills Formation in the area downgradient of the repository. This flow pattern is reflected in the pathlines plotted in Figure 5-5.

Simulated groundwater travel times are very sensitive to the distance traversed by the pathline through the Calico Hills Formation. Because the hydraulic conductivity of the Calico Hills Formation in the flow model is over two orders of magnitude lower than that in the underlying Prow Pass Tuff, there is a high contrast in particle velocities between the two units. The eastern part of the repository overlies the subcrop of the Calico Hills Formation at the water table, so particles which are initially located in this part of the model must travel through some of this lower conductivity unit and have relatively long travel times to the five $\mathrm{km}$ boundary. Particles which begin at the water table in the central portion of the region below the repository may have fairly long travel times or relatively short travel times depending on whether dispersive mixing causes them to follow a path which encounters the Calico Hills Formation or not. Particles released below the southern part of the repository are relatively quickly transported around the southern end of the Calico Hills Formation "barrier". Simulations show that the degree of transverse dispersive mixing below the water table under the central portion of the repository has a significant impact on the distribution of particle travel times from this area. 


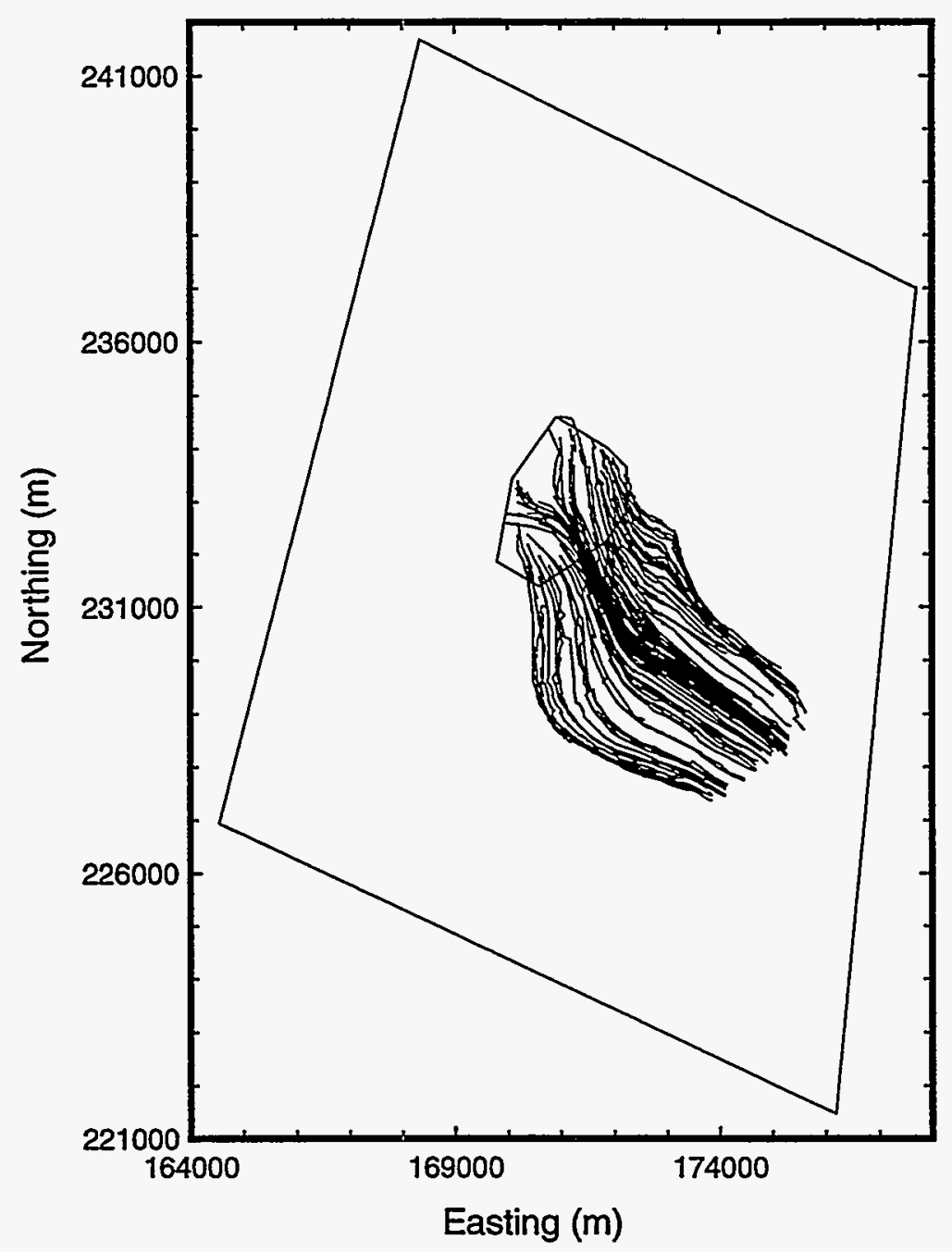

Figure 5-5 Particle pathlines from the water table below the repository to the five $\mathrm{km}$ boundary.

To quantitatively determine the distributions of simulated groundwater travel time, multiple particles (50 at each location) were tracked from individual locations on a regular grid at the water table below the repository. The results of this analysis are summarized in Figures 5-6 and 5-7. A contour map of median groundwater travel times for the region below the repository is shown in the upper part of Figure 5-6. The pattern of groundwater travel times reflects the characteristics of the simulated flow field described above, with much longer travel times occurring in the area where the Calico Hills Formation is found at the water table. The distributions of particle travel times for three example locations are shown in the CDF plot in the lower part of Figure 5-6. The distributions of travel times for location $A$ and $C$ on the CDF plot indicate approximately Gaussian dispersive spreading with relatively short and long average travel times, respectively. The distribution at location B, however, is distinctly non-Gaussian and reflects the influence of several general paths in the flow field. 

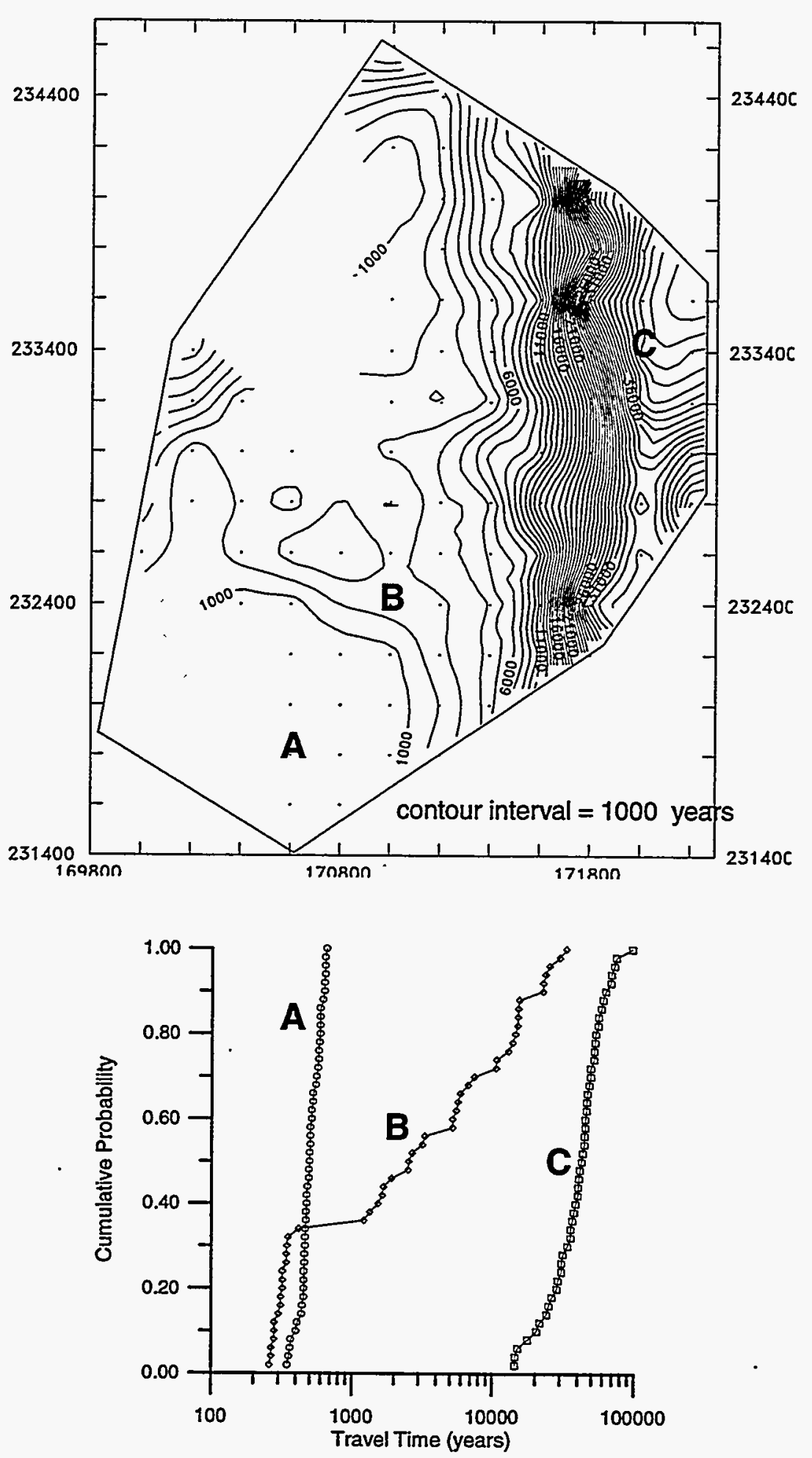

Figure 5-6 Contour map of median groundwater travel time from below the repository to the five $\mathrm{km}$ boundary. Also shown is a plot of distributions of travel time at three locations, corresponding to the locations A, B, and C on the contour map. The shaded areas indicate regions where the particle tracking method failed for some particles. The grid points on the upper figure indicate the locations of particle release. 


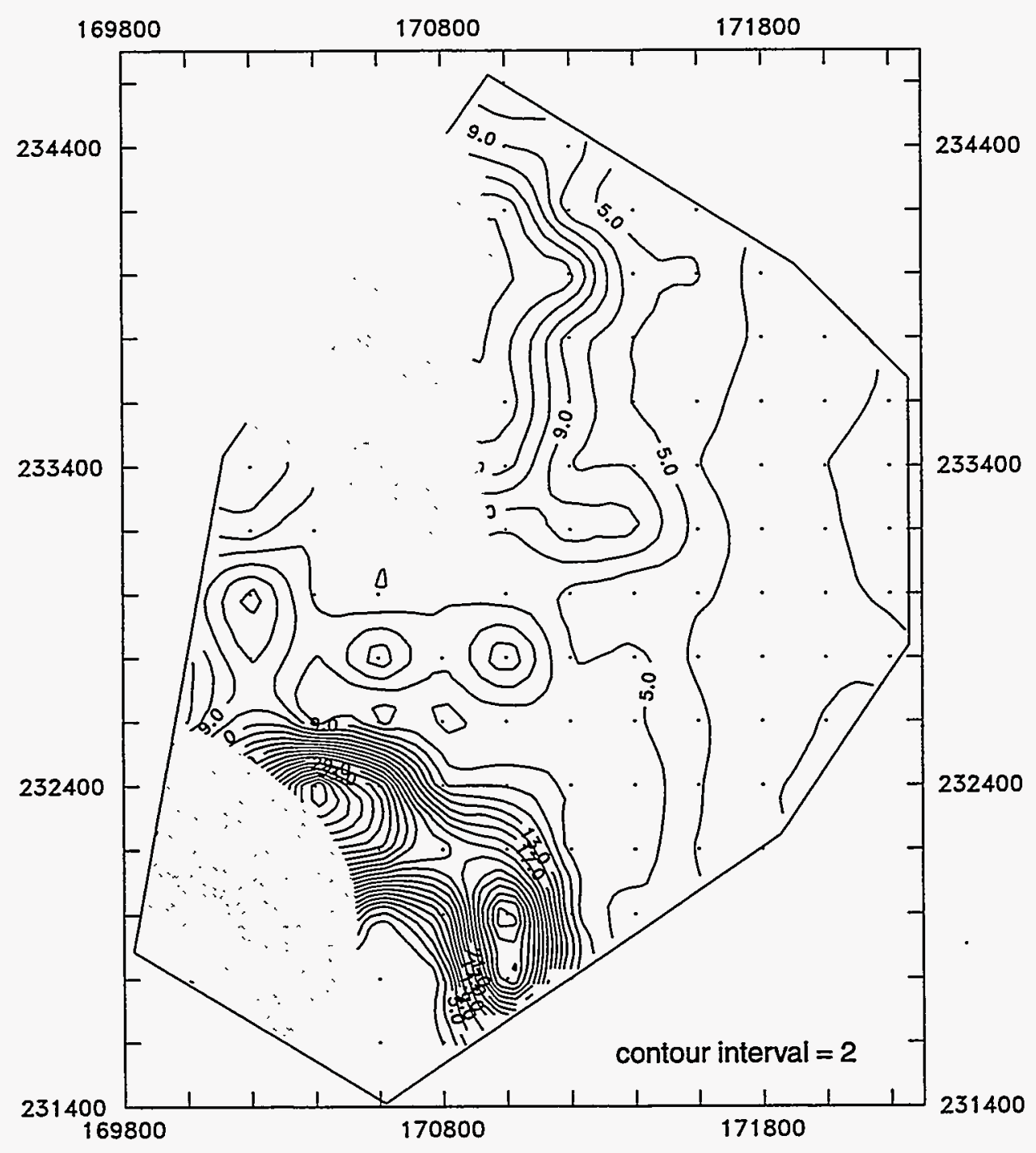

Figure 5-7 Contour map showing the variability of groundwater travel time from the water table below the repository to the five $\mathrm{km}$ boundary. The non-parametric statistic used to represent variability is the normalized interdecile range. The outline of the repository is shown. The shaded areas indicate regions where the particle tracking method failed for some particles. The grid points on the figure indicate the locations of particle release.

The particle tracking algorithm is not successful for particles initiated in two areas below the repository, as indicated by the shaded areas in Figures 5-6 and 5-7. These areas are located near the Solitario Canyon fault and splay, which are regions of very high contrast in the direction and magnitude of the hydraulic gradient in the flow model (see Figure 5-4). Due to the coarseness of the numerical grid in these areas and averaging procedures in the particle tracking method, the 
particles become trapped and cannot be tracked out of the area. The general trends in particle travel time distributions below the repository are evident in adjacent areas to indicate where the trapped particles would go.

The variability of particle travel times from beneath the repository is presented in Figure 5-7. Because of the non-uniformity in travel time distributions, a non-parametric statistic is defined to represent the variability of these distributions. The normalized interdecile range is defined as:

$$
I D R=\frac{q_{0.9}-q_{0.1}}{q_{0.5}}
$$

where $q_{0.9}$ is the ninth decile of travel time, $q_{0.1}$ is the first decile, and $q_{0.5}$ is the median. The contour map of normalized interdecile range in Figure 5-7 indicates relatively low variability in particle travel time from the areas at the water table below the eastern and extreme southern parts of the repository. An area of extreme variability in travel time is located below the southern part of the repository.

An alternative mapping of the variability in particle travel times is shown in Figure 5-8. This contour map shows the percentage of particles with travel times to the five km boundary from the position below the repository of less than 1,000 years. Only in the area at the water table below the eastern part of the repository do all of the particles have travel times in the SZ of greater than 1,000 years. At least $40 \%$ of the particles have travel times of less than 1,000 years in the areas under the north-central and southern parts of the repository. 


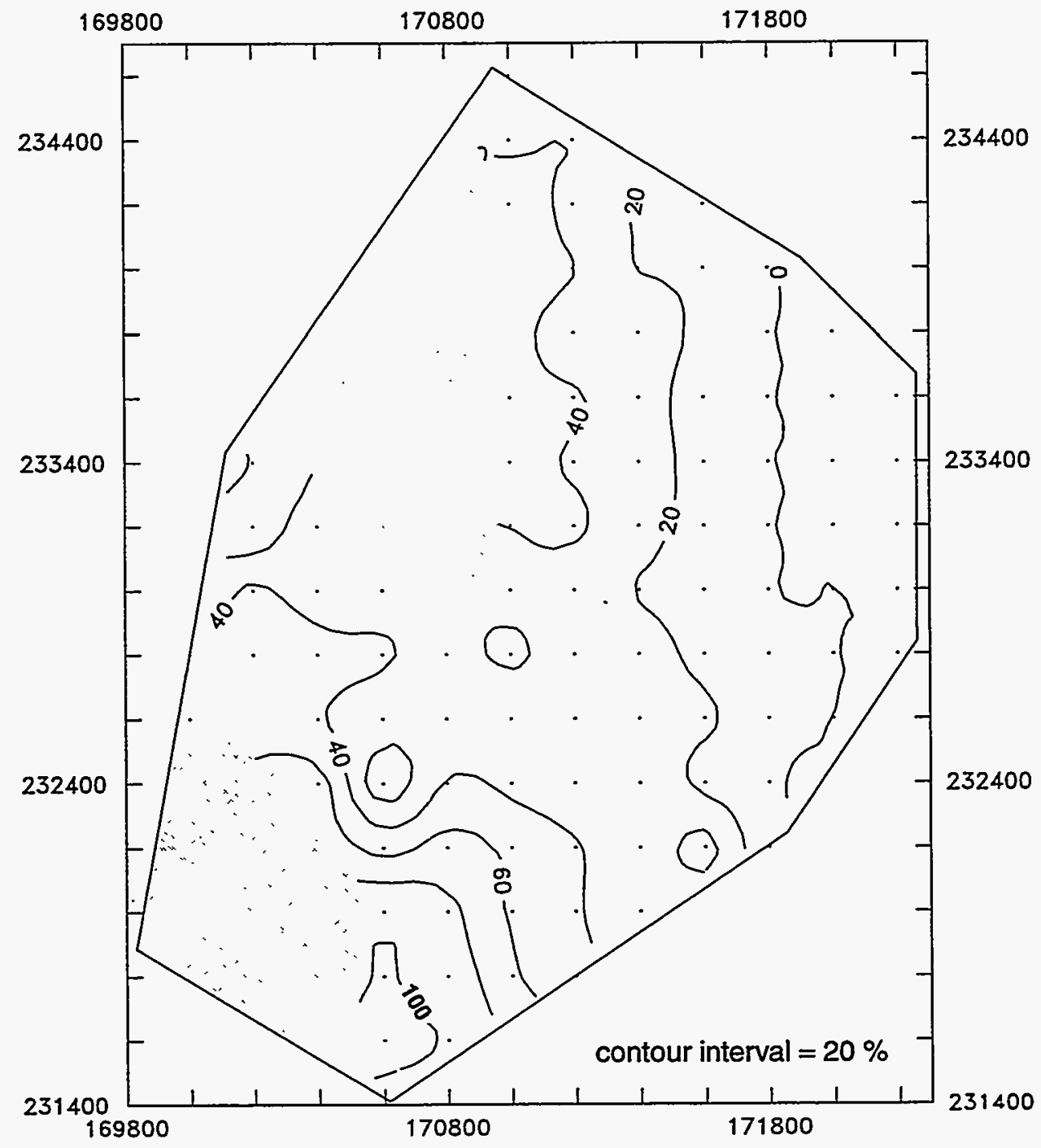

Figure 5-8 Contour map showing the percentage of particles with travel times of less than 1,000 years to the five $\mathrm{km}$ boundary. Outline of the repository is shown. The shaded areas indicate regions where particle tracking method failed for some particles. The grid points on the figure indicate the locations of particle release. 


\section{CHAPTER 6 \\ Results, Conclusions, Future Work, and Recommendations}

\subsection{Results}

Groundwater travel time work conducted for fiscal year 1995 is intended to determine travel times from the repository to the five $\mathrm{km}$ boundary of the accessible environment under prewaste emplacement conditions. The approach used divides the flow modeling and particle tracking calculations into the unsaturated and saturated zones. This chapter reports the important findings from these separate calculations and then combines the results to determine some general conclusions about total groundwater travel times. Another intention of the groundwater travel time work is to determine the effects of uncertainty in hydrological parameters on the flow regime and to attain a greater understanding of the influence of hydrological parameters which have some uncertainty on flow simulations.

\subsubsection{Unsaturated Zone}

- Through the use of geostatistical simulations linked to a geological framework model geologically reasonable hydrologic properties are successfully simulated that account for the uncertainty in the properties due to the limited available site data.

- Simulated matrix saturations compare well with those measured in core-plugs taken from boreholes. The largest discrepancies are in the $\mathrm{TCw}$, where simulated matrix saturations are lower than those measured in the core samples. The differences could be improved by introducing some of the infiltration flux into the matrix domain of the flow model instead of having the total flux infiltrate into the fracture domain.

- Simulated matrix and fracture saturations are dependent on the infiltration rate along the upper boundary, being lower under the washes than in other areas.

- Simulated fluid-flow velocity vectors in both the matrix and fracture domains are primarily vertical downwards.

- The Darcy velocities are approximately two to three orders of magnitude higher in the fracture domain than in the matrix domain. The larger flow velocities in the fractures result in the majority of particles for each simulation being tracked using the fracture flow velocities.

- Particle travel times are dependent on both the lateral location within the cross-section and geostatistical simulation. Travel times are generally longer under washes where infiltration rates, saturations and velocities are lower. Variations of one to three orders of magnitude at the same location are simulated for different geostatistical realizations. This result indicates that the uncertainty in the hydrological data has a significant effect on the calculated travel times. 
- Particle travel times appear to be dependent on the thickness of the PTn. As calculated at the southern-portion of cross-section $\mathrm{CC}$, particle travel times decrease where the PTn thins. This is thought to be due to the fact that with a thinner PTn, less water is imbibed into the matrix of the non-welded unit and, therefore, fiuxes and flow velocities are faster in the fracture domain under these areas.

- The majority of particle travel times exceed 1,000 years in the UZ. Locations where some particle travel times are less than 1,000 years are at the western portion of crosssection $\mathrm{AA}$, the eastern and central portions of cross-section $\mathrm{BB}$, throughout crosssection $\mathrm{CC}$, and at the southeast end of cross-section DD.

- Sensitivity studies demonstrate that increasing the infiltration flux at the upper boundary, the fracture-matrix connectivity, and the fracture frequency all cause an increase in matrix saturation. It is therefore possible to match measured matrix saturations by inversely adjusting the infiltration and fracture-matrix connectivity in unison. The relative influence of the different parameters is difficult to assess.

- Sensitivity studies also demonstrate that increasing the infiltration flux causes a decrease in particle travel times, in contrast to an increase in the fracture-matrix connectivity and fracture frequency which cause an increase in particle travel times. Changes in infiltration flux appear to have the greatest effect on the particle travel times with approximately order of magnitude changes in travel times with order of magnitude changes in infiltration rates. Fracture-matrix connectivity has a greater effect on the particle travel times at lower infiltration rates.

- Both the equivalent continuum and dual permeability models are able to match measured core saturations. Use of the ECM, however, results in significantly longer particle travel times.

- Simulation of lateral flow in the system is dependent on both the conceptual model used (ECM vs. DKM) and the matrix van Genuchten $\alpha$. Lateral flow in the PTn occurs with the ECM and van Genuchten parameters used in previous studies (Klavetter and Peters, 1986).

- The application of a large transient pulse of infiltration along the upper boundary causes flow velocities in the fracture domain to increase throughout the mountain, though the relative increase decreases with depth. This pulse of increased velocity causes a water particle released at the surface to reach the PTn within one year of the initiation of the pulse, which is consistent with recent isotopic dating.

\subsubsection{Sāturated Zone}

- The saturated zone flow model is calibrated to the 27 available hydraulic head measurements and the inferred direction and magnitude of the hydraulic gradient by decreasing the hydraulic conductivity at the Solitario Canyon fault, Solitario Canyon fault splay, Drill Hole Wash structure and the Yucca Wash structure. 
- Simulated particle travel times from the water table to the five $\mathrm{km}$ boundary of the accessible environment are sensitive to the geometry of the hydrostratigraphic units, specifically the low-conductivity Calico Hills formation. Pathlines through this formation result in travel times over two-orders of magnitude longer than pathlines through other units.

- In general, particles released at the water table at the eastern portion of the repository have travel times greater than 10,000 years whereas many particles released below the southern and western portions of the repository have travel times less than 1,000 years.

\subsubsection{Combined Unsaturated and Saturated Zone Travel Times}

The addition of particle travel times from the unsaturated and saturated zone is not straightforward. First, UZ calculations are made along two-dimensional cross-sections, whereas the particles are tracked in three dimensions in the SZ model. In addition, at any given location in the UZ there are 10 calculated travel times based on the different geostatistical realizations. On the other hand, in the SZ, only one deterministic geological model is used, yet 50 particles are released at a given location which take different paths due to hydrodynamic and numerical dispersion. For these reasons, combined UZ-SZ particle travel times are evaluated only at several general locations.

As a qualitative method of assessing combined UZ-SZ particle travel times, distributions for both the UZ and SZ along UZ cross-sections AA and CC are presented in Figure 6-1a and 61b. Examination of Figure 6-1a shows that where some particle travel times are less than 1,000 years in the UZ (the eastern portion of the cross-section), the travel times exceed 1,000 years in the SZ. However, in the central portion of both the unsaturated and saturated zones, particles have travel times of less than 1,000 years. Therefore, it is unlikely that a particle released along this cross-section would arrive at the accessible environment in less than 1,000 years except in the central portion of the cross-section. It is more difficult to qualitatively assess the combined distributions of travel time along cross-section CC because there are some travel times less than 1,000 years in both the UZ and the SZ along most of the length of this cross-section.

In order to obtain a more quantitative assessment of combined UZ-SZ groundwater travel times, cumulative distribution functions (CDF's) of particle travel times are estimated from five locations within the potential repository (in the north, west, south, and east comers, and in the center) (Figure 6-2). The CDF's were constructed by taking the 50 particles released at the water table in the SZ calculations and randomly adding them to 50 particles from the UZ calculations released at the same lateral locations (see Figures 6-3 and 6-4). Only at the southern end of the repository are some of the total particle travel times less than 1,000 years (Figure 6-3a). At this location approximately $60 \%$ of the travel times exceed 1,000 years. Particle travel times are the longest in the eastern portion of the potential repository where all combined travel times exceed 10,000 years. Particle travel times are the longest from the eastern portion of the potential repository, primarily due to the extremely long travel times calculated in the SZ (Figure 6-1a). At the western, central and northern portions of the repository the fastest particle travel times range from approximately 1,000 to 5,000 years (Figure 6-3). An assumption in randomly adding travel times in the $\mathrm{UZ}$ and $\mathrm{SZ}$ is that travel times in the two domains are independently distributed. This 
UZ Particle Travel Times, Section AA, DK Model

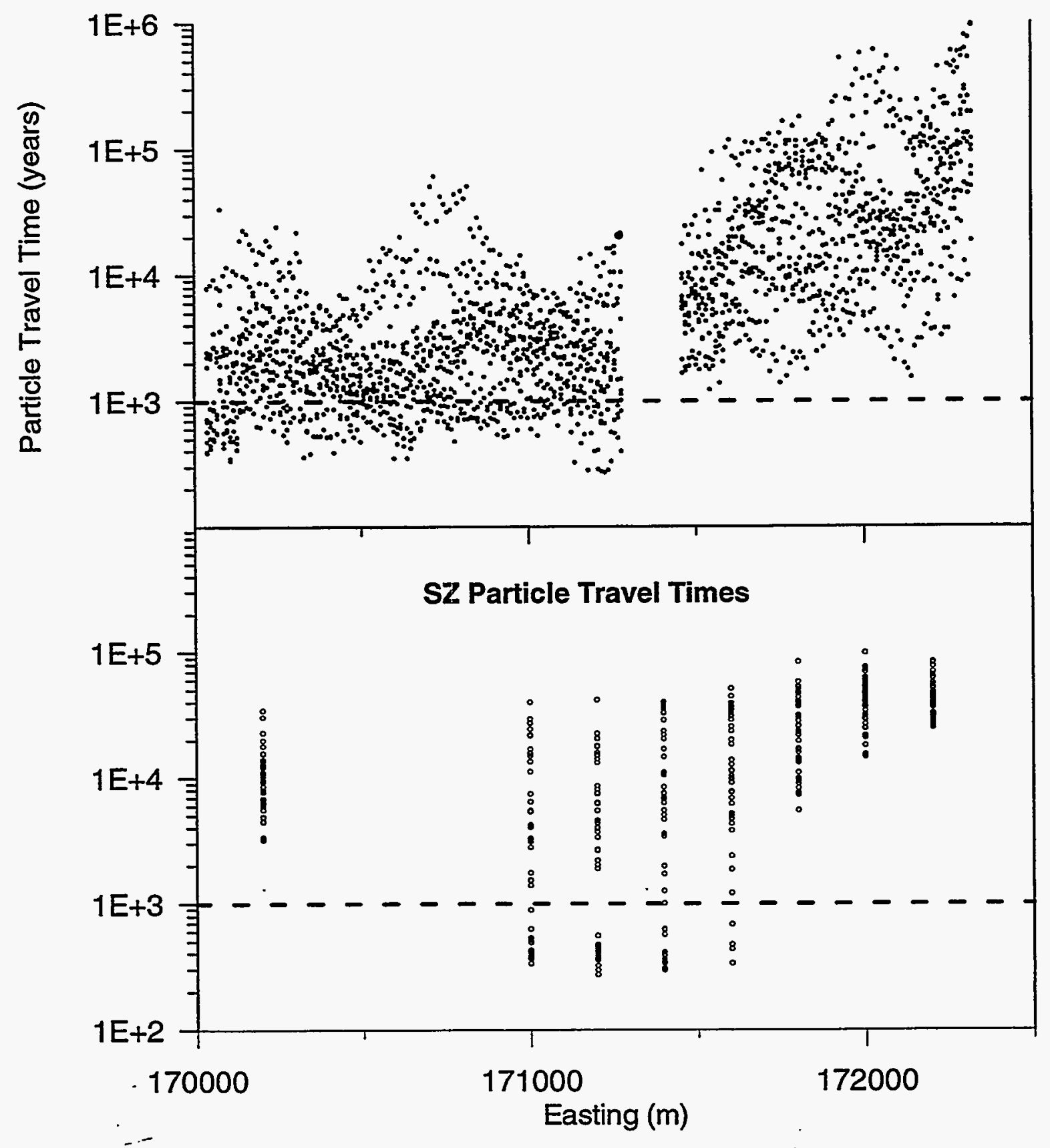

Figure 6-1a Combined unsaturated and saturated zone particle travel times along crosssection AA. 


\section{UZ Particle Travel Times, Section CC, DK Model}

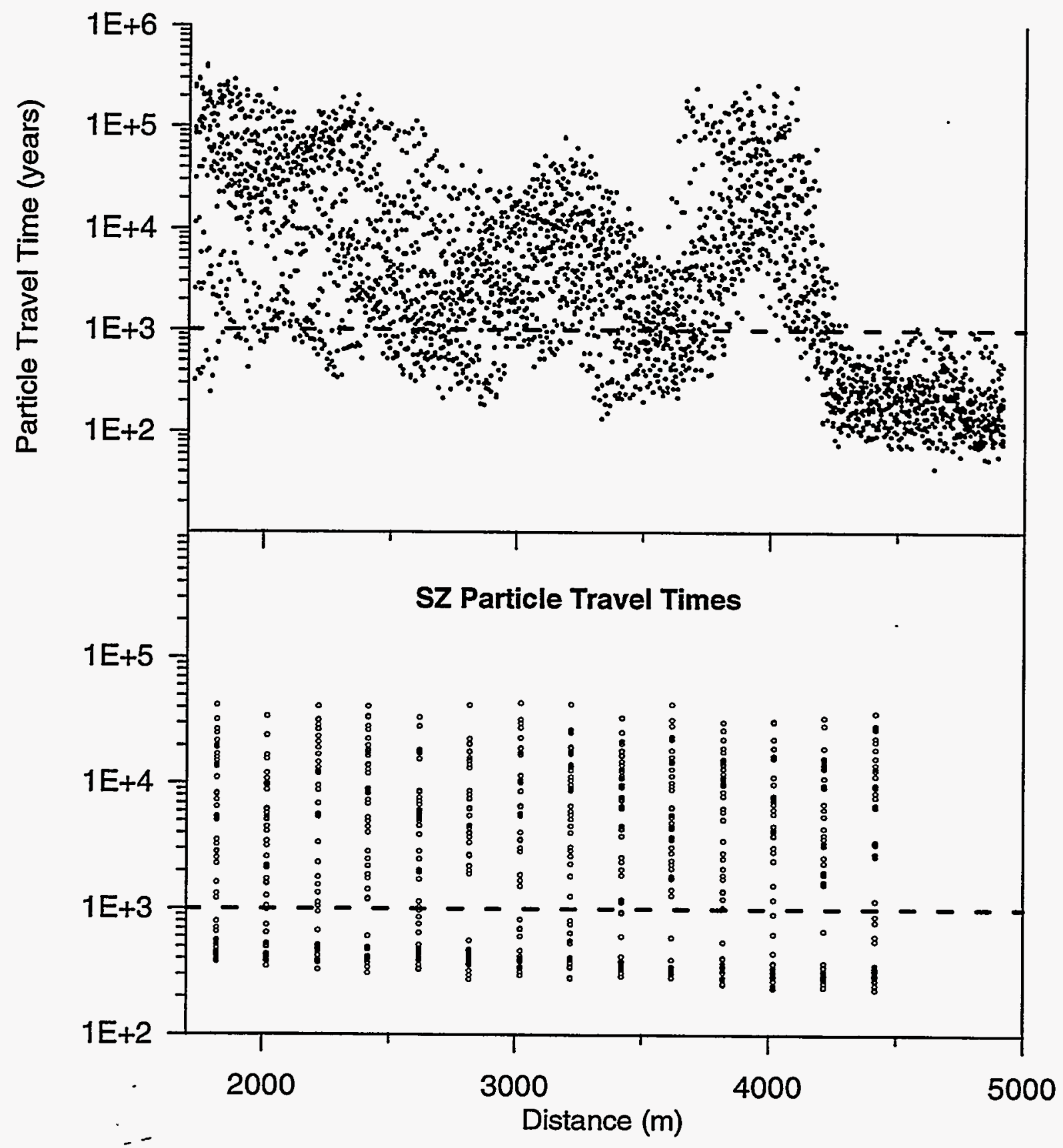

Figure 6-1b Combined unsaturated and saturated zone particle travel times along crosssection CC. 
implies that fast pathways in the UZ are not preferentially connected to fast pathways in the SZ.

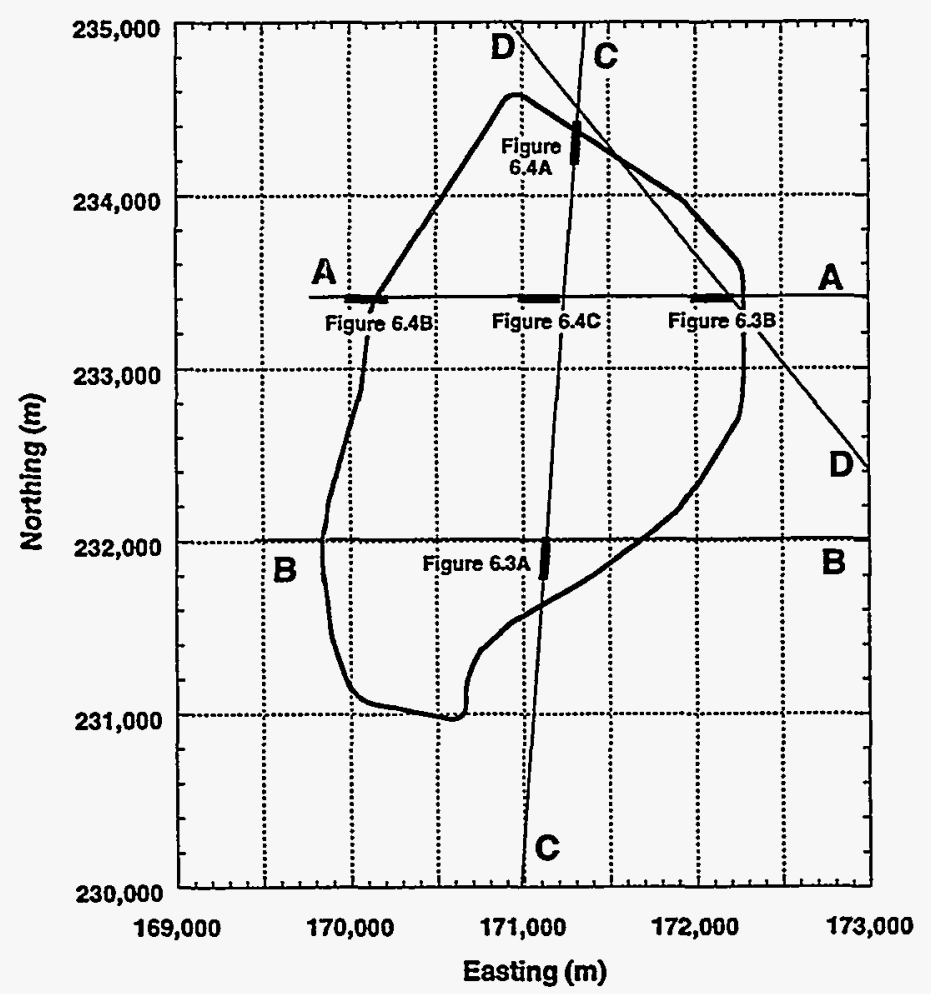

Figure 6-2 Locations within the potential repository where cumulative probability functions were determined for groundwater travel times from the repository horizon to the accessible environment.

\subsection{Conclusions}

The results of this study demonstrate the importance of the conceptual model of flow processes used in these analyses. The DKM is more physically realistic than the ECM used in past modeling studies as it allows for disequilibrium in capillary pressure between the fractures and matrix. Observed distributions of matrix saturations are matched using the DKM. In addition, the DKM simulates relatively faster groundwater flow in the fracture domain. clearly an important process for fast-path flow.

Comparison of the results among multiple geostatistical simulations demonstrates the value of incorporating heterogeneity in material properties into the analyses. The inclusion of uncertainty and heterogeneity in material properties in the UZ flow simulations results in variability of approximately one to three orders of magnitude in simulated groundwater travel times at a given location.

Simulations of groundwater flow are particularly sensitive to the infiltration flux at the ground surface, which has a strong control on both saturations and flow velocities of the system. Particle travel times decrease by approximately one order of magnitude with an order of 

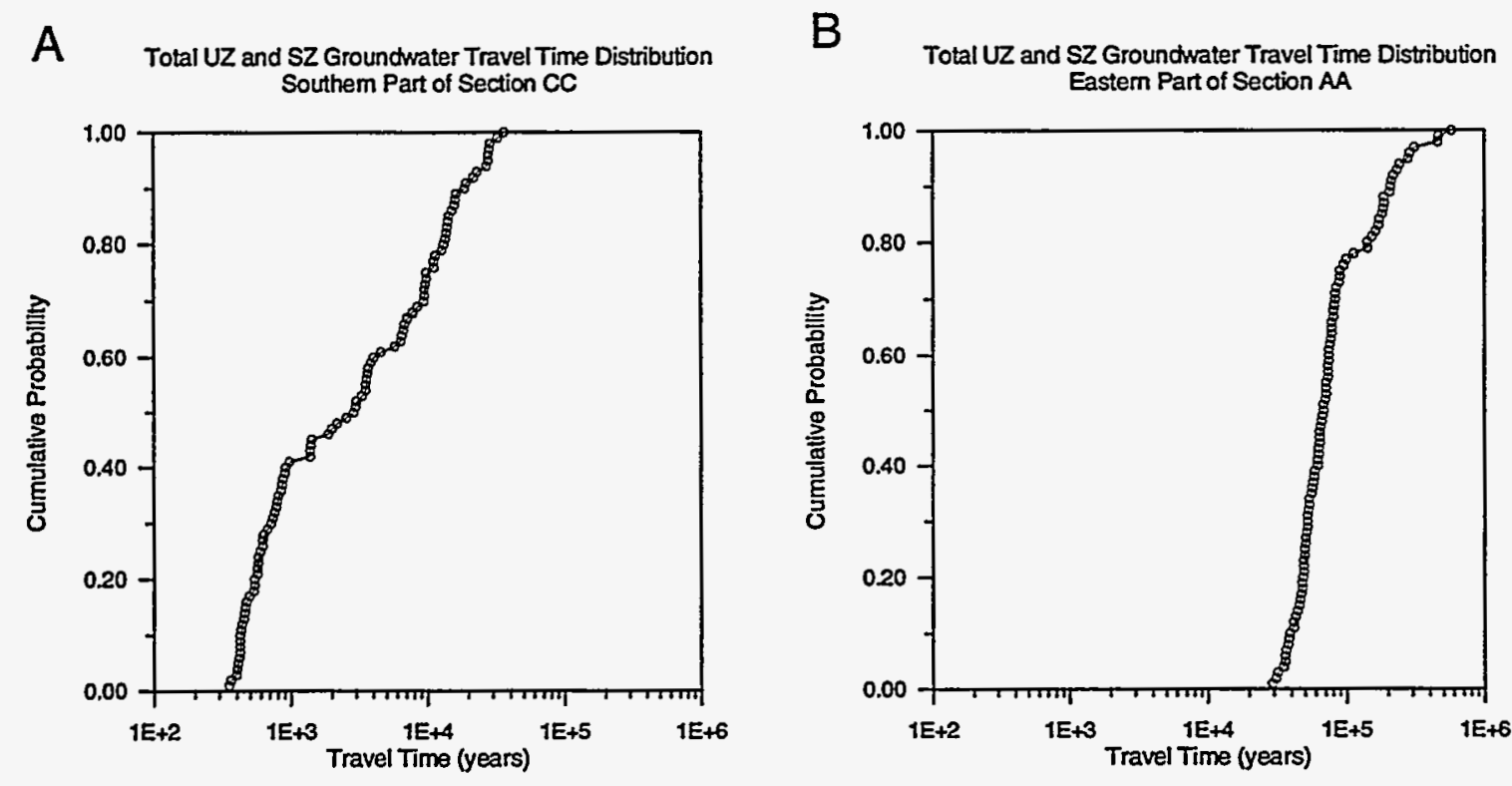

Figure 6-3 CDF's of combined unsaturated-zone saturated-zone particle travel times for particle released in the southern (A) and eastern (B) portions of the potential repository footprint (see Figure 6-2 for locations).

magnitude increase in mean infiltration. Groundwater flow simulations are also sensitive to poorly constrained physical parameters describing the interaction between unsaturated groundwater flow in fractures and the matrix, fracture-matrix connectivity. In-situ saturations can be matched by inversely adjusting the infiltration and fracture-matrix connectivity, both parameters with significant uncertainty. In theory, therefore, it could be possible to decrease the infiltration and increase the interaction between the fracture and matrix domains and still simulate realistic saturations. These simulations, however, would lead to much longer particle travel times (greater than 1,000 years).

The particle tracking method used to differentiate between flow in the matrix and fractures in the UZ modeling represents a conservative approximation with significant influence on the calculated distributions of groundwater travel times in the UZ. As explained in Section 4.4.2, particle movement is tracked at the higher of the two simulated velocities in the matrix and fractures, which in most areas is in the fracture continuum (see Figures 4-9a to 4-9d). However, a much greater volume of groundwater is contained in the matrix than in the fractures at any location in the UZ. The distribution of travel times calculated by this method is thus not a complete distribution of travel times in both the fractures and matrix; it excludes some of the longer travel times resulting from flow in the matrix. Therefore, it should be noted when interpreting the groundwater travel time distribution results for the UZ (and the combined travel 

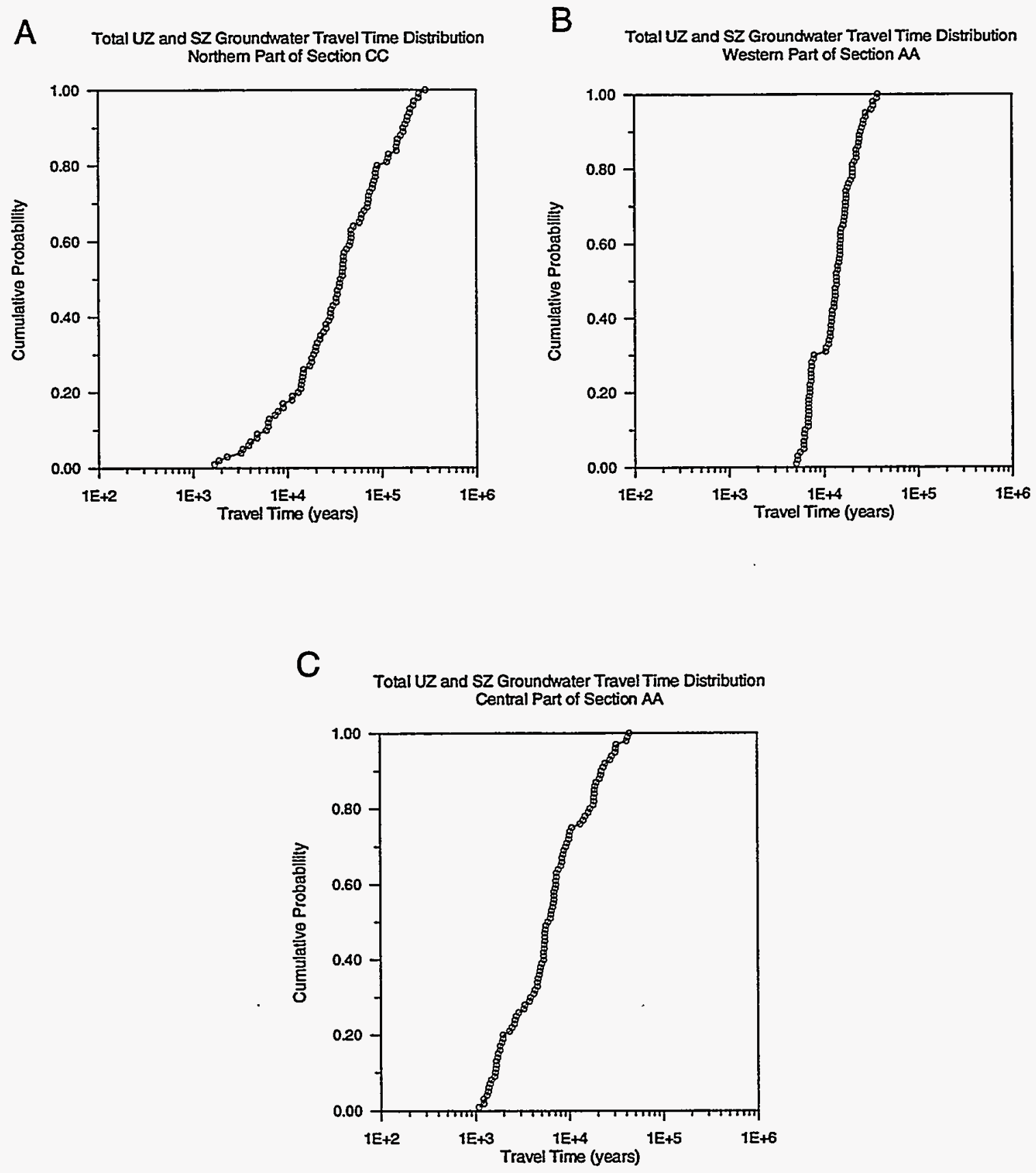

Figure 6-4 CDF's of combined unsaturated-zone saturated-zone particle travel times for particle released in the northern (A), western (B) and central (C) portions of the potential repository footprint (see Figure 6-2 for locations). 
time distributions) that while these results capture the fastest simulated travel times they do not represent complete distributions of travel times.

\subsection{Implications With Regard To GWTT Regulations}

The results of the analyses in this study indicate that total simulated groundwater travel times exceed 1,000 years for particle releases from the potential repository at four of the five representative locations considered. However, approximately $40 \%$ of the particles released from one location at the southern portion of the repository have total travel times of less than the regulatory limit of 1,000 years (Figure 6-3).

Both the NRC Performance Requirement and the DOE Disqualifying Condition as outlined in Section 1.1 stipulate that groundwater travel time is to be considered along pathways of "likely" radionuclide travel. Flow through fractures in the UZ to the water table, as addressed in this study, is considered to be the most likely pathway for transport of radionuclides by groundwater flow in current conceptual models of unsaturated flow at Yucca Mountain. The nature of the groundwater flow simulations in this study and the resulting groundwater travel time distributions thus satisfy the criterion of likelihood specified in the regulations. The DOE Disqualifying Condition regulation also states that travel times are to be considered only along paths of "significant" radionuclide travel. Use of the "significant" qualifier suggests that the fraction of groundwater travel times faster than 1,000 years can be used as a measure of the significance of those faster flow paths. That $40 \%$ of the simulated groundwater travel times from the southern part of the repository are less than 1,000 years would probably be considered "significant" under this interpretation of the regulations. The term "significant" can also be interpreted to refer to the amount of radionuclides that could be transported along these pathways. Under this interpretation, the significance of a particular flow path would be weighed in relation to possible mass flux, which would have to be addressed through additional transport modeling within the simulated flow field. There are important uncertainties in this analysis of groundwater travel time, especially in the UZ, that preclude definitive evaluation of regulatory compliance.

The implications of the groundwater travel time calculations reported in this document in relation to the regulatory criteria should be interpreted in light of the uncertainties and limitations of the analysis. Sensitivity analyses reported in Section 4.5 indicate that particle travel times may vary several orders of magnitude in the UZ flow simulations within plausible ranges of some input parameters. Values of infiltration flux and fracture-matrix connectivity exert particularly great influence on modeled groundwater travel time distributions. Sensitivity analysis also suggests that a finer numerical grid results in longer travel times. The distributions of groundwater travel times reported in Section 6.1.3 for the base case analysis should not be viewed as "the" answer to the question of GWTT, but should be considered within the context of the associated sensitivity studies. The limitations of the conservative approximation of exchange of flow between fractures and matrix in the UZ particle tracking method described in Section 6.2 and its consequent overemphasis of the fastest travel times should be considered also. Finally, the influence of the "disturbed zone" on particle travel times has not been considered in this study. 


\subsection{Future Directions}

The next step in evaluating groundwater travel time distributions is to assess the effect of the repository on the flow regime. Groundwater travel time simulations to date have all been "pre-waste emplacement" calculations. That is, the hydrologic parameters are assumed to be the same as what are presently being measured. It is also assumed that there is not a heat source at the repository. However, as stated in Chapter 1, post-waste emplacement calculations are necessary to account for the "disturbed zone" mentioned in the regulations. Therefore, work planned for to account for the "disturbed zone" mentioned in the regulations. Therefore, work planned for FY96 will include post-waste emplacement calculations. This modeling work will use the same crosssections and methods for simulating hydrologic properties described in this report. The difference is that thermo-hydrologic properties will be simulated and a heat source will be added to the repository horizon. A different grid will be used that is more refined at the repository horizon. The three-dimensional saturated zone model will also be enhanced to include thermal modeling.

\subsection{Recommendations}

One of the most significant findings of this study is that the fastest travel times in both the unsaturated and saturated zones are in the southern portion of the potential repository. Yet, this is an area that is not well constrained by site data (see Figure 3.1). Simulated travel times in the unsaturated zone under this portion of the potential repository vary by approximately one to three orders of magnitude due to uncertainty in the hydrological properties. It is more difficult to assess the effect of uncertainty on the saturated zone calculations. Nevertheless, it is recommended that site-characterization studies concentrate on the southern portion of the potential repository.

Groundwater travel times are also found to be sensitive to many parameters. Specifically, infiltration through the upper boundary has a strong effect on the simulated saturations and travel times. While it is difficult to accurately determine the present-day infiltration into the bedrock, better constrained values will yield more accurate numerical modeling predictions. Similarly, the demonstration that matrix saturations and particle travel times are also sensitive to changes in fracture-matrix connectivity demonstrate the importance of attaining a better understanding of fracture-matrix interactions through laboratory, field and numerical experiments. The non-unique calibration of matching simulated to measured saturation by inversely adjusting infiltration and fracture matrix connectivity further support the importance of better constraining these parameters and also using different performance measures to conduct calibrations.

The understanding of whether lateral flow occurs in the PTn is critical to evaluating the flux at the potential repository horizon. Sensitivity studies show that lateral flow can be simulated in the non-welded PTn unit through variation of the van Genuchten $\alpha$ parameter and conceptual model (ECM). The existence of lateral flow is still uncertain. Field studies need to be conducted to determine whether there is evidence for lateral flow in the PTn. It is also important to better constrain the van Genuchten $\alpha$ parameter at the flow modeling scale since it has been shown to have a strong influence on the simulation of lateral flow. Numerical studies constraining the range of parameters needed to simulate lateral flow would also be useful. 
An additional mechanism which may influence the flux of groundwater in the UZ that is not considered in this study is vapor phase transport by natural convection of gas. If significant amounts of groundwater are removed from the mountain by this process it could have an important impact on the overall groundwater flow system in the UZ. Additional numerical modeling of multiphase flow is recommended to assess the significance of this process. A reduction in groundwater flux in the UZ by this mechanism would result in slower velocities in fractures and longer groundwater travel times.

Distributions of groundwater travel times in the SZ are sensitive to the conceptual model of flow and to the values and variability of hydraulic conductivity. The assumption of groundwater exchange between fractures and matrix must be substantiated by tracer experiments in the field. Relative groundwater travel times in the SZ have been established in this study, but the absolute values of travel times can only be accurately determined by acquiring additional data on the hydraulic conductivity. Field testing of wells to confirm or deny the presence of discrete zones of preferential groundwater flow (e.g. fault zones) is required to bound the possible focusing of SZ flow. 
This page intentionally left blank. 


\section{References}

Arnold, B.W., S.J. Altman, T.H. Robey, R.W. Barnard, and T.J. Brown. 1995. Unsaturated-Zone Fast-Path Flow Calculations for Yucca Mountain Groundwater Travel Time Analyses (GWTT-94). SAND95-0857. Albuquerque, NM: Sandia National Laboratories. 169 p.

Barnard, R.W., M.L. Wilson, H.A. Dockery, J.H. Gauthier, P.G. Kaplan, R.R. Eaton, F.W. Bingham and T.H. Robey. 1992. TSPA 1991: An Initial Total-System Performance Assessment for Yucca Mountain. SAND91-2795. Albuquerque, NM: Sandia National Laboratories. 386 p.

Barton, C.C., E. Larsen, W.R. Page, and T.M. Howard. 1993. Characterizing Fractured Rock for Fluid-Flow, Geomechanical, and Paleostress Modeling: Methods and Preliminary Results for Yucca Mountain, Nevada. USGS/OFR-93-269. Denver, CO: U.S. Geological Survey.

Bish, D.L., and S.J. Chipera. 1989. Revised Mineralogic Summary of Yucca Mountain, Nevada. LA-11497-MS. Los Alamos, NM: Los Alamos National Laboratory.

Bloomsburg, G., R.E. Williams, and J.L. Osiensky. 1989. "Distribution of Downward Flux in Unsaturated Heterogeneous Hydrogeology Environments," Geological Society of America Bulletin. Vol. 101, no. 12, 1623-1630.

Chekuri, V.S., S.W. Tyler, and J.W. Foordham. 1994. "The Role of Fracture Coatings on Water Imbibition into Unsaturated Tuff," High Level Radioactive Waste Management, Proceedings of the Fifth Annual International Conference, Las Vegas, Nevada, May 22-26, 1994. La Grange Park, IL: American Nuclear Society. 1891-1896.

Chipera, S.J., D.T. Vaniman, B.A. Carlos, and D.L. Bish. 1995. Mineralogic Variation in Drill Core UE-25 UZ\#16, Yucca Mountain, Nevada. LA-12810-MS. Los Alamos, NM: Los Alamos National Laboratory.

Czarnecki, J.B. 1985. Simulated Effects of Increased Recharge on the Ground-Water Flow System of Yucca Mountain and Vicinity, Nevada-California. WRI-84-4344. Denver, CO: U.S. Geological Survey. 38 p.

Czarnecki, J.B. 1989. "Preliminary Simulations Related to a Large Horizontal Hydraulic Gradient at the North End of Yucca Mountain, Nevada," EOS, Transactions of the American Geophysical Union. Vol. 70, no. 15, 321.

Czarnecki, J.B. and R.K. Waddell. 1984. Finite-Element Simulation of Ground-Water Flow in the Vicinity of Yucca Mountain, Nevada-California. Water Resources Investigations Report 84-4349. Denver, CO:-U.S. Geological Survey. 42 p.

Czarnecki, J.B. and R.R. Luckey. 1989. "Regional and Local Flow Systems near Yucca Mountain Site, Nevada," Geological Society of America 1989 Field Trip Guidebook. J.D. Vineyard and W.K. Wedge (comps.). Rolla, MO: Missouri Department of Natural Resources. Special Publication No. 5, 32-33. 
D’Agnese, F. A. 1994. Using Geoscientific Information Systems for Three-Dimensional Modeling of Regional Ground-Water Flow Systems, Death Valley Region, Nevada and California.

Unpublished Ph.D. Dissertation. Golden, CO: Colorado School of Mines, Department of Geology and Geological Engineering. 331 p.

Department of Energy (DOE). 1984. "Nuclear Waste Policy Act of 1982; General Guidelines for the Recommendation of Sites for the Nuclear Waste Repositories; Final Siting Guidelines," Code of Federal Regulations, Title 10, Part 960. Washington, DC: U.S. Government Printing Office, Superintendent of Documents.

Desbarats, A.J. 1995. "Upscaling Capillary Pressure-Saturation Curves in Heterogeneous Porous Media," Water Resources Research. Vol. 31, no. 2, 281-288.

Deutsch, C.V. and A.G. Journel. 1992. GSLIB: Geostatistical Software Library and User's Guide. New York: Oxford University Press. 340 pp.

Dudley, A.L., R.R. Peters, J.H. Gauthier, M.L. Wilson, M.S. Tierney and E.A. Klavetter. 1988. Total System Performance Assessment Code (TOSPAC) Vol. 1 Physical and Mathematical Bases. SAND85-0002. Albuquerque, NM: Sandia National Laboratories. 212 p.

Eaton, R.R., C.K. Ho, R.J. Glass, M.J. Nicholl, and B.W. Arnold. 1996. "Modeling of Flow Through Fractured Tuff at Fran Ridge," High Level Radioactive Waste Management, Proceedings of the Seventh Annual International Conference, Las Vegas, Nevada, April 29 - May 3, 1996. SAND95-2618A. La Grange Park, IL: American Nuclear Society. 76-78.

Ervin, E.M., R.R. Luckey, and D.J. Burkhardt. 1993. "Summary of Revised PotentiometricSurface Map for Yucca Mountain and Vicinity, Nevada," High Level Radioactive Waste Management, Proceedings of the Fourth Annual International Conference, Las Vegas, Nevada, April 26-30, 1993. La Grange Park, IL: American Nuclear Society. Vol. 2, 1554-1558.

Faunt, C.C. 1994. Characterization of the Three-Dimensional Hydrogeologic Framework of the Death Valley Region, Nevada and California. Unpublished Ph.D. Dissertation. Golden, CO: Colorado School of Mines, Department of Geology and Geological Engineering. 256 p.

Flint, L.E. and A.L. Flint. 1990. Preliminary Permeability and Water-Retention Data for Nonwelded and Bedded Tuff Samples, Yucca Mountain Area, Nye County, Nevada. USGS/OFR90-569. Denver, CO: U.S. Geological Survey. 62 p.

Flint, A.L. and L.E. Flint. 1994. "Spatial Distribution of Potential Near Surface Moisture Flux at Yucca Mountain," High Level Radioactive Waste Management, Proceedings of the Fifth Annual International Conference, Las Vegas, Nevada, May 22-26, 1994. La Grange Park, IL: American Nuclear Society. Vol. 4, 2352-2358.

Flint, L.E., Ā.L. Flint, and J.A. Hevesi. 1994. "Shallow Infiltration Processes in Arid Watersheds at Yucca Mountain, Nevada." High Level Radioactive Waste Management, Proceedings of the Fifth Annual International Conference, Las Vegas, Nevada, May 22-26, 1994. La Grange Park, IL: American Nuclear Society. Vol. 4, 2315-2322. 
Flint, L.E., A.L. Flint, C.A. Rautman, and J.D. Istok. 1996. Physical and Hydrologic Properties of Rock Outcrop Samples at Yucca Mountain, Nevada. USGS/OFR-95-280. Denver, CO: U.S. Geological Survey.

Freeze, R.A. and J.A. Cherry. 1979. Groundwater. Englewood Cliffs, NJ: Prentice-Hall, Inc. $604 \mathrm{p}$.

Fridrich, C.J., W.W. Dudley, Jr. and J.S. Stuckless. 1994. "Hydrogeologic Analysis of the Saturated-Zone Ground-Water System Under Yucca Mountain, Nevada," Journal of Hydrology. Vol. 154 , no. 1-4, 133-168.

Geldon, A.L. 1993. Preliminary Hydrogeologic Assessment of Boreholes UE-25c \#1, UE-25c \#2, and UE-25c \#3, Yucca Mountain, Nye County, Nevada. Water Resources Investigations Report 92-4016. Denver, CO: U.S. Geological Survey.

Gelhar, L.W., A.L. Gutjahr, and R.L. Naff. 1979. "Stochastic Analysis of Macrodispersion in a Stratified Aquifer," Water Resources Research. Vol. 15, no. 6, 1387-1397.

Gelhar, L.W. and C. L. Axness. 1983. "Three-Dimensional Stochastic Analysis of Macrodispersion in Aquifers," Water Resources Research. Vol. 19, no. 1, 161-180.

Gelhar, L.W., A. Mantoglou, C. Welty, and K.R. Rehfeldt. 1985. A Review of Field-Scale Physical Solute Transport Processes in Saturated and Unsaturated Porous Media. EPRI EA4190 Project 2485-5. Palo Alto, CA: Electric Power Research Institute. 116 p.

Glass, R.J. and V.C. Tidwell. 1991. Research Program to Develop and Validate Conceptual Models for Flow and Transport Through Unsaturated, Fractured Rock. SAND90-2261. Albuquerque, NM: Sandia National Laboratories. $70 \mathrm{p}$.

Hevesi, J.A., A.L. Flint, and J.D. Istok. 1992. "Precipitation Estimation in Mountainous Terrain Using Multivariate Geostatistics. Part II: Isohyetal Maps," Journal of Applied Meteorology: Vol. 31 , no. 7, 677-688.

Hevesi, J.A. and A.L. Flint. 1993. "The Influence of Seasonal Climatic Variability on Shallow Infiltration at Yucca Mountain," High Level Radioactive Waste Management, Proceedings of the Fourth Annual International Conference, Las Vegas, Nevada, April 26-30, 1993. La Grange Park, IL: American Nuclear Society. 122-131.

Ho, C.K., S.J. Altman, and B.W. Amold. 1995. Alternative Conceptual Models and Codes for Unsaturated Flow in Fractured Tuff: Preliminary Assessments for GWTT-95. SAND95-1546. Albuquerque, NM: Sandia National Laboratories. 138 p.

Huyakorn, P S., S. Panday, and A. Sinha. 1992. STAFF3D-- A Three-Dimensional Finite Element Code for Simulating Fluid Flow and Transport of Radionuclides in Fractured Porous Media with Water Table Boundary Conditions, Version 2.0. Herndon, VA: HydroGeoLogic, Inc. 300 p.

Istok, J.D., C.A. Rautman, L.E. Flint, and A.L. Flint. 1994. "Spatial Variability in Hydrologic Properties of a Volcanic Tuff," Groundwater. Vol. 32, no. 5, 751-760. 
Journel, A.G. and Ch.J. Huijbregts. 1978. Mining Geostatistics. New York: Academic Press. $600 \mathrm{p}$.

Kaplan, P.G. 1993. Pre-Waste Emplacement Ground-Water Travel Time Sensitivity and Uncertainty Analyses for Yucca Mountain, Nevada. SAND92-0461. Albuquerque, NM: Sandia National Laboratories. $121 \mathrm{p}$.

Klavetter, E.A. and R.R. Peters. 1986. Estimation of Hydrologic Properties of an Unsaturated, Fractured Rock Mass. SAND84-2642. Albuquerque, NM: Sandia National Laboratories. 51 p.

Kume, J. and J.P. Rousseau. 1994. "A Borehole Instrumentation Program for Characterization of Unsaturated-Zone Percolation," High Level Radioactive Waste Management, Proceedings of the Fifth Annual International Conference, Las Vegas, Nevada, May 22-26, 1994. La Grange Park, IL: American Nuclear Society. 2076-2083.

LeCain, G.D. and J.N. Walker. 1994. "Results of Air-Permeability Testing in a Vertical Borehole at Yucca Mountain, Nevada," High Level Radioactive Waste Management, Proceedings of the Fifth Annual International Conference, Las Vegas, Nevada, May 22-26, 1994. La Grange Park, IL: American Nuclear Society. 2782-2788.

Lin, Y.T. and M.S. Tierney. 1986. Preliminary Estimates of Groundwater Travel Time and Radionuclide Transport at the Yucca Mountain Repository Site.. Ed. S. Sinnock. SAND85-2701. Albuquerque, NM: Sandia National Laboratories. 84 p.

Lu, N. 1994. "A Semianalytical Method of Path Line Computation for Transient FiniteDifference Groundwater Flow Models," Water Resources Research. Vol. 30, no. 8, 2449-2559.

Luster, G. R. 1985. Raw Materials for Portland Cement: Applications of Conditional Simulation of Coregionalization. Ph.D. Dissertation. Stanford, CA: Stanford University, Department of Applied Earth Sciences. 531 p.

McKenna, S.A. and C.A. Rautman. 1995. Summary Evaluation of Yucca Mountain Surface Transects with Implications for Downhole Sampling. SAND94-2038. Albuquerque, NM: Sandia National Laboratories. $45 \mathrm{pp}$.

McKenna, S.A. and C.A. Rautman. 1996. Scaling of Properties for Yucca Mountain: Literature Review and Numerical Experiments on Saturated Hydraulic Conductivity. SAND95-2338. Albuquerque, NM: Sandia National Laboratories.

Montazer, P. and W.E. Wilson. 1986. "Hydrogeology of the Unsaturated Zone, Yucca Mountain, Nevada," Proceedings of the NWWA Conference on Characterization and Monitoring of the Vadose (Unsaturated) Zone, Denver, Colorado, November 19-21, 1985. Worthington, OH: National Water Well Association. 396-412.

Moyer, T.C. and J.K. Geslin. 1995. Lithostratigraphy of the Calico Hills Formation and Prow Pass Tuff (Crater Flat Group) at Yucca Mountain, Nevada. USGS/OFR-94-460. Reston, VA: U.S. Geological Survey. 
Nicholl, M.J. and R.J. Glass. 1995. "Effective Media Models for Unsaturated Fractured Rock: A Field Experiment," High Level Radioactive Waste Management 1995, Proceedings of the Sixth Annual International Conference, Las Vegas, Nevada, April 30-May 5, 1995. La Grange Park, IL: American Nuclear Society. 39-40.

Nitao, J.J. and T.A. Buscheck. 1991. "Infiltration of a Liquid Front in an Unsaturated Fractured Porous Medium," Water Resources Research. Vol. 27, no. 8, 2099-2112.

Nuclear Regulatory Commission (NRC). 1983. "Disposal of High-Level Radioactive Wastes in Geologic Repositories," Code of Federal Regulations, Title 10, Part 60. Washington, DC: U.S. Government Printing Office, Superintendent of Documents.

Peters, R.R., E.A. Klavetter, I.J. Hall, S.C. Blair, P.R. Heller, and G.W. Gee. 1984. Fracture and Matrix Hydrogeologic Characteristic of Tuffaceous Materials from Yucca Mountain, Nye County, Nevada. SAND84-1471. Albuquerque, NM: Sandia National Laboratories. 184 p.

Peters, R.R., E.A. Klavetter, J.T. George, and J.H. Gauthier. 1987. "Measuring and Modeling Water Imbibition into Tuff," Flow and Transport Through Unsaturated Fractured Rock Media, Geophysical Monograph 42, D.D. Evans and T.J. Nicholson, eds., Washington, DC: American Geophysical Union. 99-106.

Pollock, D.W. 1988. "Semianalytical Computation of Path Lines for Finite Difference Models," Ground Water. Vol. 26, no. 6, 743-750.

Prindle, R.W. and P.L. Hopkins. 1990. On Conditions and Parameters Important to Model Sensitivity for Unsaturated Flow through Layered, Fractured Tuff: Results of Analyses for HYDROCOIN Level 3 Case 2. SAND89-0652. Albuquerque, NM: Sandia National Laboratories. $252 \mathrm{p}$.

Pruess, K. 1983. GMINC-A Mesh Generator for Flow Simulations in Fractured Reservoirs. LBL-15227. Berkeley, CA: Lawrence Berkeley Laboratory.

Pruess, K. 1987. TOUGH User's Guide. LBL-20700. Berkeley, CA: Lawrence Berkeley Laboratory. $88 \mathrm{p}$.

Pruess, K. 1991. TOUGH2--A General Purpose Numerical Simulator for Multiphase Fluid and Heat Flow. LBL-29400. Berkeley, CA: Lawrence Berkeley Laboratory. 102 p.

Rautman, C.A. 1991. Estimates of Spatial Correlation in Volcanic Tuff, Yucca Mountain, Nevada. SAND89-2270. Albuquerque, NM: Sandia National Laboratories.

Rautman, C.A. 1994. "Development of Stochastic Indicator Models of Lithology, Yucca Mountain, Nevada," High Level Radioactive Waste Management, Proceedings of the Fifth Annual International Conference, Las Vegas, Nevada, May 22-26, 1994. La Grange Park, IL: American Nuclear Society. 2510-2519.

Rautman, C.A. 1995. Preliminary Geostatistical Modeling of Thermal Conductivity for a Cross Section of Yucca Mountain, Nevada. SAND94-2283. Albuquerque, NM: Sandia National Laboratories. $64 \mathrm{p}$. 
Robey, T.H. 1994. Development of Models for Fast Fluid Pathways Through Unsaturated Heterogeneous Porous Media. SAND93-7109. Albuquerque, NM: Sandia National Laboratories.

Robinson, B.A. 1994. "A Strategy for Validating a Conceptual Model for Radionuclide Migration in the Saturated Zone Beneath Yucca Mountain," Radioactive Waste Management and Environmental Restoration. Vol. 19, no. 1-3, 73-96.

Robison, J.H. 1984. Ground-Water Level Data and Preliminary Potentiometric-Surface Maps, Yucca Mountain and Vicinity, Nye County, Nevada. U.S. Geological Survey Water Resources Investigations Report 84-4197. Denver, CO: U.S. Geological Survey.

Rutherford, B.M., I.J. Hall, R.R. Peters, R.G. Easterling, and E.A. Klavetter. 1992. Statistical Analysis of Hydrologic Data for Yucca Mountain. SAND87-2380. Albuquerque, NM: Sandia National Laboratories.

Sass, J.H., A.H. Lachenbruch, W.W. Dudley, Jr., S.S. Priest, and R.J. Munroe. 1988.

Temperature, Thermal Conductivity, and Heat Flow Near Yucca Mountain, Nevada: Some Tectonic and Hydrologic Implications. U.S. Geological Survey Open-File Report 87-649. Denver, CO: U.S. Geological Survey.

Schenker, A.R., D.C. Guerin, T.H. Robey, C.A. Rautman, and R.W. Barnard. 1995. Stochastic Hydrogeologic Units and Hydrogeologic Properties Development for Total-System Performance Assessments. SAND94-0244. Albuquerque, NM: Sandia National Laboratories. 194 p.

Scott, R.B., R.W. Spengler, S. Diehl, A.R. Lappin, and M.P. Chornack. 1983. "Geologic Character of Tuffs in the Unsaturated Zone at Yucca Mountain, Southern Nevada," Role of the Unsaturated Zone in Radioactive and Hazardous Waste Disposal. J.W. Mercer, P.S.C. Rao and I.W. Marine (eds.), Ann Arbor, MI: Ann Arbor Science. 289-335.

Scott, R.B. and J. Bonk. 1984. Preliminary Geologic Map of Yucca Mountain, Nye Count;, Nevada, with Geologic Sections. USGS/OFR- 84-494. Denver, CO: U.S. Geological Survey.

Scott, R.B. and M. Castellanos. 1984. Stratigraphic and Structural Relations of Volcanic Rocks in Drill Holes USW GU-3 and USW G-3, Yucca Mountain, Nye County, Nevada. USGS/OFR84-491. Denver, CO: U.S. Geological Survey. 121 p.

Scott, R.B. 1990. "Tectonic Setting of Yucca Mountain, Southwest Nevada," Basin and Range Extensional Tectonics Near the Latitude of Las Vegas, Nevada. B.P. Wernicke (ed.). Memoir 176. Boulder, CO: Geological Society of America. 251-282.

Sinnock, S., Y.T. Lin, and J.P. Brannen. 1984. Preliminary Bounds on the Expected Postclosure Performance of the Yucca Mountain Repository Site, Southern Nevada. SAND84-1492. Albuquerque, NM: Sandia National Laboratories.

Sinton, P.O. 1989. "Characterization of the Large Hydraulic Gradient Beneath the North End of Yucca Mountain, Nevada," EOS Transactions, American Geophysical Union. Vol. 70, no. 15, 321. 
Spengler, R.W., F.M. Byers, Jr., and J.B. Warner. 1981. Stratigraphy and Structure of Volcanic Rocks in Drill Hole USW-G1, Yucca Mountain, Nye County, Nevada. USGS/OFR-81-1349. Denver, CO: U.S. Geological Survey.

Spengler, R.W., M.P. Chornack, D.C. Muller, and J.E. Kibler. 1984. Stratigraphic and Structural Characteristics of Volcanic Rocks in Core Hole USW G-4, Yucca Mountain, Nye County, Nevada. USGS/OFR-84-789. Denver, CO: U.S. Geological Survey.

Thoma, S.G., D.P. Gallegos, and D.M. Smith. 1990. Effect of Mineral Layers on Capillary Uptake from Fracture Surfaces: Implications for Waste Disposal in Unsaturated, Fractured Rock: Letter report submitted to U.S. NRC Office of Nuclear Regulatory Research, July, 1990.

Tidwell, V.C. 1994. "Laboratory Investigation of Constitutive Property Scaling Behavior" Formation Evaluation and Reservoir Geology, Proceedings of the 1994 SPE Annual Technical Conference and Exhibition, New Orleans, LA, September 25-28, 1994. SPE 28456, SAND94 1745C. Richardson, TX: Society of Petroleum Engineers. 947-957.

Van Genuchten, M.Th. 1980. "A Closed-Form Equation for Predicting the Hydraulic Conductivity of Unsaturated Soils," Soil Science Society of America Journal. Vol. 44, no. 5, 892898.

Voss, C. 1993. "New Moisture Retention Curves." Letter to T. Robey, dated February 15, 1993, Golder Associates, Inc., Redmond, WA. (NNA.940113.0033)

Wang, J.S.Y., and T.N. Narasimhan. 1985. "Hydrologic Mechanisms Governing Fluid Flow in a Partially Saturated Fractured Porous Medium," Water Resources Research. Vol. 21, no. 12, 18611874.

Wang, J.S.Y., and T.N. Narasimhan. 1986. Hydrologic Mechanisms Governing Partially Saturated Fluid Flow in Fractured, Welded Units and Porous, Nonwelded Units at Yucca Mountain. SAND85-7114, LBL-21022. Albuquerque, NM: Sandia National Laboratories; Berkeley, CA: Lawrence Berkeley Laboratory.

Wang, J.S.Y., and T.N. Narasimhan. 1988. Hydrologic Modeling of Vertical and Lateral Movement of Partially Saturated Fluid Flow Near a Fault Zone at Yucca Mountain. SAND877070, LBL-23510. Albuquerque, NM: Sandia National Laboratories; Berkeley, CA: Lawrence Berkeley Laboratory.

Warren, J.E. and P.J. Root. 1963. "The Behavior of Naturally Fractured Reservoirs," Society of Petroleum Engineers Journal. Vol. 3, no. 5, 245-255.

Wilson, M.L. 1996. "Lateral Diversion in the PTn Unit: Capillary-Barrier Analysis," High Level Radioactive Waste Management, Proceedings of the Seventh Annual International Conference, Las Vegas, Nevada, April 29 - May 3, 1996. SAND95-2186C. La Grange Park, IL: American Nuclear Society. 111-113. 
Wilson, M. L., J. H. Gauthier, R.W. Barnard, G.E. Barr, H.A. Dockery, E. Dunn, R.R. Eaton, D.C. Guerin, N. Lu, M.J. Martinez, R. Nilson, C.A. Rautman, T.H. Robey, B. Ross, E.E. Ryder, A.R. Schenker, S.A. Shannon, L.H. Skinner, W.G. Halsey, J.D. Gansemer, L.C. Lewis, A.D. Lamont, I.R. Triay, A. Meijer and D.E. Morris. 1994. Total-System Performance Assessment for Yucca Mountain - SNL Second Iteration (TSPA-1993). SAND93-2675. Albuquerque, NM: Sandia National Laboratories.

Winograd, I.J., and W. Thordarson. 1975. Hydrogeologic and Hydrochemical Framework, Southcentral Great Basin, Nevada-California, with Special Reference to the Nevada Test Site.

Professional Paper 712-C. Washington, DC: U.S. Geological Survey.

Wittwer, C., G. Chen, G.S. Bodvarsson, M. Chornack, A. Flint, L. Flint, E. Kwicklis, and R. Spengler. 1995. Preliminary Development of the LBL-USGS Three-Dimensional Site-Scale Model of Yucca Mountain, Nevada. LBL-37356. Berkeley, CA: Lawrence Berkeley Laboratory. 


\section{YUCCA MOUNTAIN SITE CHARACTERIZATION PROJECT SAND96-0819 - DISTRIBUTION LIST}

1

D. A. Dreyfus (RW-1)

Director

OCRWM

US Department of Energy

1000 Independence Avenue SW

Washington, DC 20585

1 L. H. Barrett (RW-2)

Acting Deputy Director OCRWM

US Department of Energy

1000 Independence Avenue SW

Washington, DC 20585

1 S. Rousso (RW-40)

Office of Storage and Transportation

OCRWM

US Department of Energy

1000 Independence Avenue SW

Washington, DC 20585

1 R. A. Milner (RW-30)

Office of Program Management

and Integration

OCRWM

US Department of Energy

1000 Independence Avenue SW

Washington, DC 20585

1 D. R. Elle, Director

Environmental Protection Division

DOE Nevada Field Office

US Department of Energy

P.O. Box 98518

Las Vegas, NV 89193-8518

1 T. Wood (RW-14)

Contract Management Division

OCRWM

US Department of Energy

1000 Independence Avenue SW

Washington, DC 20585

4 Victoria F. Reich, Librarian

Nuclear Waste Technical Review Board 1100 Wilson Blvd., Suite 910

Arlington, VA 22209

1 Wesley Barnes, Project Manager

Yucca Mountain Site Characterization Office

US Department of Energy

P.O. Box 98608--MS 523

Las Vegas, NV 89193-8608
1 Director, Public Affairs Office

c/o Technical Information Resource Center DOE Nevada Operations Office

US Department of Energy

P.O. Box 98518

Las Vegas, NV 89193-8518

8 Technical Information Officer

DOE Nevada Operations Office

US Department of Energy

P.O. Box 98518

Las Vegas, NV 89193-8518

1 J. R. Dyer, Deputy Project Manager

Yucca Mountain Site Characterization Office

US Department of Energy

P.O. Box 98608 - MS 523

Las Vegas, NV 89193-88608

M. C. Brady

Laboratory Lead for YMP

$\mathrm{M} \& \mathrm{O} /$ Sandia National Laboratories

1261 Town Center Drive

BIdg. 4, Room 421A

Las Vegas, NV 89134

1

J. A. Canepa

Laboratory Lead for YMP

EES-13, Mail Stop J521

$\mathrm{M} \& \mathrm{O} / L$ os Alamos National Laboratory

P.O. Box 1663

Los Alamos, NM 87545

1 Repository Licensing \& Qualit

Assurance

Project Directorate

Division of Waste Management. MIS T7J.9

US NRC

Washington, DC 20555

1 Senior Project Manager for Yucca

Mountain

Repository Project Branch

Division of Waste Management, MS T7J-9

US NRC

Washington, DC 20555

1 NRC Document Control Desk

Division of Waste Management, MS T7J-9

US NRC

Washington, DC 20555 
1

Chad Glenn

NRC Site Representative

301 E Stewart Avenue, Room 203

Las Vegas, NV 89101

1 Center for Nuclear Waste

Regulatory Analyses

Southwest Research Institute

6220 Culebra Road

Drawer 28510

San Antonio, TX 78284

2 W. L. Clarke

Laboratory Lead for YMP

M\&O/ Lawrence Livermore Nat'I Lab

P.O. Box 808 (L-51)

Livermore, CA 94550

1 Robert W. Craig

Acting Technical Project Officer/YMP

US Geological Survey

101 Convention Center Drive, Suite P-110

Las Vegas, NV 89109

1 J. S. Stuckless, Chief

Geologic Studies Program

MS 425

Yucca Mountain Project Branch

US Geological Survey

P.O. Box 25046

Denver, CO 80225

1 L. D. Foust

Technical Project Officer for YMP

TRW Environmental Safety Systems

101 Convention Center Drive

Suite P-110

Las Vegas, NV 89109

1 A. L. Flint

U. S. Geological Survey MS 721

P. O. Box 327

Mercury, NV 89023

$1 \quad$ Robert L. Strickler

Vice President \& General Manager

TRW Environmental Safety Systems, Inc.

2650 Park Tower Dr.

Vienna, VA 22180

1 Jim Krulik, Geology Manager

US Bureau of Reclamation

Code D-8322

P.O. Box 25007

Denver, CO 80225-0007
1

B. T. Brady

Records Specialist

US Geological Survey

MS 421

P.O. Box 25046

Denver, CO 80225

1

M. D. Voegele

Deputy of Technical Operations

M\&O/SAIC

101 Convention Center Drive

Suite P-110

Las Vegas, NV 89109

2 A. T. Tamura

Science and Technology Division

OSTI

US Department of Energy

P.O. Box 62

Oak Ridge, TN 37831

1 P. J. Weeden, Acting Director Nuclear Radiation Assessment Div.

US EPA

Environmental Monitoring Sys. Lab

P.O. Box 93478

Las Vegas, NV 89193-3478

1 John Fordham, Deputy Director

Water Resources Center

Desert Research Institute

P.O. Box 60220

Reno, NV 89506

1 The Honorable Jim Regan

Chairman

Churchill County Board of

Commissioners

$10 \mathrm{~W}$. Williams Avenue

Fallon, NV 89406

1

R. R. Loux

Executive Director

Agency for Nuclear Projects

State of Nevada

Evergreen Center, Suite 252

1802 N. Carson Street

Carson City, NV 89710

1

Brad R. Mettam

County Yucca Mountain

Repository Assessment Office

P. O. Drawer L

Independence, CA 93526 
1 Vernon E. Poe

Office of Nuclear Projects

Mineral County

P.O. Box 1600

Hawthome, NV 89415

1 Les W. Bradshaw

Program Manager

Nye County Nuclear Waste Repository

Project Office

P.O. Box 1767

Tonopah, NV 89049

1 Florindo Mariani

White Pine County Coordinator

P. O. Box 135

Ely, NV 89301

1 Tammy Manzini

Lander County Yucca Mountain

Information Officer

P.O. Box 10

Austin, NV 89310

1 Jason Pitts

Lincoln County Nuclear Waste

Program Manager

P. O. Box 158

Pioche, NV 89043

1 Dennis Bechtel, Coordinator

Nuclear Waste Division

Clark County Dept. of Comprehensive

Planning

P.O. Box 55171

Las Vegas, NV 89155-1751

1 Juanita D. Hoffman

Nuclear Waste Repository

Oversight Program

Esmeralda County

P.O. Box 490

Goldfield, NV 89013

$1 \quad$ Sandy Green

Yucca Mountain Information Office

Eureka County

P.O. Box 714

Eurreka, NV 89316

1 Economic Development Dept.

City of Las Vegas

400 E. Stewart Avenue

Las Vegas, NV 89101

1 Community Planning \& Development

City of North Las Vegas

P.O. Box 4086

North Las Vegas, NV 89030
2 Librarian

YMP Research \& Study Center

101 Convention Center Drive, Suite P-110

Las Vegas, NV 89109

1 Library Acquisitions

Argonne National Laboratory

Building 203, Room CE-111

9700 S. Cass Avenue

Argonne, II 60439

1 Glenn Van Roekel

Manager, City of Caliente

P.O. Box 158

Caliente, NV 89008

1 Gudmundur S. Bodvarsson

Head, Nuclear Waste Department

Lawrence Berkeley National Laboratory

1 Cyclotron Road, MS 50E

Berkeley, CA 94720

1 Steve Hanauer (RW-2)

OCRWM

U. S. Department of Energy

1000 Independence Ave.

Washington, DC 20585

Robert W. Clayton

M\&O/WCFS

101 Convention Center Drive/MS423

Las Vegas, NV 89109

1 Richard C. Quitmeyer

M\&O/WCFS

101 Convention Center Drive/MS423

Las Vegas, NV 89109

1 Mark C. Tynan

DOE/YMPSCO

101 Convention Center Drive/MS523/HI

Las Vegas, NV 89109

2 Robert W. Andrews

M\&O/Intera

1261 Town Center Drive

MS 423

Las Vegas, NV 89134

1 S. David Sevougian

M\&O/Intera

1261 Town Center Drive

MS 423

Las Vegas, NV 89134

1 Srikanta Mishra

Intera

6850 Austin Center Blvd.

Suite 300

Austin, TX 78731 
R. G. Baca

Center for Nuclear Waste

Regulatory Analyses

6220 Culebra Road, Bldg. 189

San Antonio, TX 78238-5166

1

Neil M. Coleman

Division of Waste Management

Office of NMSS

U.S. Nuclear Regulatory Commission

Washington, DC 20555

$1 \quad$ Lorraine Flint

U.S. Geological Survey

P.O. Box 327

Mercury, NV 89023

1 Ed Kwicklis

U.S. Geological Survey

P.O. Box 25046

MS 421

Lakewood, CO 80225

$1 \quad$ Dwight Hoxie

U.S. Geological Survey

101 Convention Center Drive

MS 423/527

Las Vegas, NV 89109

1 James Duguid

Intera

2650 Park Tower Drive

Suite 800

Vienna, VA 22180

1 Steven H. Hanauer

U.S. Department of Energy

1000 Independence Avenue

RW-2

Washington, DC 20584

1 Andy Wolfsberg

Los Alamos National Laboratory

P.O. Box 1663/J521

Los Alamos, NM 87545

1 J. Fabryka-Martin

Los Alamos National Laboratory

P.O. Box 1663

Group CST-7, MS J514

Los Alamos, NM 87545

$1 \quad$ George Zyvoloski

Los Alamos National Laboratory

P.O. Box 1663

J521

Los Alamos, NM 87545
1 Bruce Robinson

Los Alamos National Laboratory

P.O. Box 1663

J521

Los Alamos, NM 87545

$1 \quad$ Mark Bandurraga

Lawrence Berkeley Laboratory

1 Cyclotron Road, MS 50E

Berkeley, CA 94720

$2 \quad$ Yu-Shu Wu

Lawrence Berkeley Laboratory

1 Cyclotron Road, MS 50E

Berkeley, CA 94720

$1 \quad$ Yvonne Tsang

Lawrence Berkeley Laboratory

1 Cyclotron Road,

MS 50-A-1149

Berkeley, CA 94720

1 Dwayne Chesnut

Lawrence Livermore National Laboratory

P.O. Box 808

MS L-202

Livermore, CA 94550

$1 \quad$ Mark Bakker

Department of Civil Engineering

500 Pillsbury Drive SE

Minneapolis, MN 55455

1

Anna Littleboy

United Kingdom Nirex Limited

Curie Avenue

Harwell, Didcot

Oxfordshire, OX11 ORH

United Kingdom

1330 B. Pierson, 6811

100/1.2.5.4.4/SAND96-0819/NQ

$201330 \quad$ WMT Library, 6752

9018

Central Technical Files, 8523-2

0899 Technical Library, 4414

0619 Review and Approval Desk, 12630, For DOE/OSTI

0835 R. R. Eaton, 9112

1324 P. B. Davies, 6115

1324 C. K. Ho, 6115

1324 S. A. McKenna, 6115

1324 C. A. Rautman, 6115

1326 S. J. Altman, 6851

1326 B. W. Arnold, 6851

1326 R. W. Barnard, 6851

1326 G. E. Barr, 6851 
$1 \quad 1326 \quad$ H. A. Dockery, 6851

$11326 \quad$ N. D. Francis, 6851

1. 1326 J. H. Gauthier, 6851

11326 S. A. Shannon, 6851

$1 \quad 1326$ M. L. Wilson, 6851

Distribution - 5 